\title{
Vertical stiffness characterization of a geocomposite drainage layer for PCC highway pavements
}

\author{
Joseph G. Sweet \\ West Virginia University
}

Follow this and additional works at: https://researchrepository.wvu.edu/etd

\section{Recommended Citation}

Sweet, Joseph G., "Vertical stiffness characterization of a geocomposite drainage layer for PCC highway pavements" (2005). Graduate Theses, Dissertations, and Problem Reports. 1947.

https://researchrepository.wvu.edu/etd/1947

This Thesis is protected by copyright and/or related rights. It has been brought to you by the The Research Repository @ WVU with permission from the rights-holder(s). You are free to use this Thesis in any way that is permitted by the copyright and related rights legislation that applies to your use. For other uses you must obtain permission from the rights-holder(s) directly, unless additional rights are indicated by a Creative Commons license in the record and/ or on the work itself. This Thesis has been accepted for inclusion in WVU Graduate Theses, Dissertations, and Problem Reports collection by an authorized administrator of The Research Repository @ WVU. For more information, please contact researchrepository@mail.wvu.edu. 


\title{
Vertical Stiffness Characterization of a Geocomposite Drainage Layer for PCC Highway Pavements
}

\author{
Joseph G. Sweet \\ Thesis Submitted to the \\ College of Engineering and Mineral Resources \\ at West Virginia University \\ in Partial Fulfillment of the Requirements \\ for the Degree of: \\ Master of Science \\ in \\ Civil Engineering \\ Roger H.L. Chen, Ph.D., Chair \\ Hota V. GangaRao, Ph.D. \\ Felicia F. Peng, Ph.D. \\ Department of Civil and Environmental Engineering \\ Morgantown, West Virginia \\ 2005
}

Keywords: Geocomposite, Rigid Pavements, Highway Pavement Design, Highway Drainage 


\section{ABSTRACT \\ Vertical Stiffness Characterization of a Geocomposite Drainage Layer for PCC Highway Pavements}

\section{Joseph G. Sweet}

One method for providing proper drainage for Portland Cement Concrete (PCC) highway pavement systems could be incorporating a geocomposite which creates a thin layer of voids through which water can flow. This research was done to develop a method for accounting for such a material during the thickness design of the PCC pavement. A theoretical method for predicting the results of field plate bearing tests of a soil system was adapted to include a geocomposite. Also, the vertical stiffness behavior of a particular geocomposite was characterized in the laboratory via uniaxial compression and cyclic fatigue tests. The validity of the theoretical method was verified by comparing deflections calculated using these vertical stiffness characteristics to experimental data collected from plate bearing tests, as well as to results of Finite Element (FE) models. Good compatibility was shown between plate deflections calculated theoretically and those obtained using the Finite Element method. 


\section{ACKNOWLEDGEMENTS}

I would like to thank my advisor and the chairman of my advisory committee, Dr. Roger H. L. Chen, for giving me the opportunity to conduct this research project, as well as for the support and guidance provided throughout this study. Also, gratitude is extended to the other members of the advisory committee, Dr. Hota V. GangaRao and Dr. Felicia F. Peng, for reviewing this work and for providing constructive comments and suggestions pertaining to this research.

Appreciation is also extended to Tenax Corporation for providing the financial support and the specimens which were used for the laboratory tests. Without their interest in this particular field this research project would not have been pursued.

A debt of gratitude goes to my officemates and co-workers who provided support in many respects during this project. In particular, the work of Dr. Alejandro Kiriakidis, throughout the planning and execution of the numerous laboratory tests was invaluable to this research. Also, the time and effort put forth by Mr. Jeong Hoon Choi, Mr. Tuan-Chun (Jimmy) Fu, and Mr. Chee Heong Tee during the laboratory investigations were greatly appreciated. The preliminary data and feedback provided by Mr. Joshua Cook was also very helpful in giving this research direction.

I would also like to thank Mr. Bill Comstock and Mr. Jerry Nestor for their assistance with the setup and instrumentation of the laboratory experiments. Also, the knowledge and assistance of Ricardo Kiriakidis with respect to laboratory soil testing was very helpful in performing that particular aspect of the research.

Last, but certainly not least, I would like to thank my family, especially my parents, Ronald and Wendie Sweet, as well as my siblings, Ron, Taunya, and Kyle, and my nieces and nephews, as well as my friends for all of the patience and encouragement they've shown for me, not only throughout this project, but throughout the course of my life.

This work also constitutes the final report submitted to the Tenax Corporation. 


\section{TABLE OF CONTENTS}

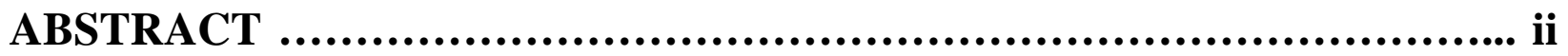

ACKNOWLEDGEMENTS...$\ldots \ldots \ldots \ldots \ldots \ldots \ldots \ldots \ldots \ldots \ldots \ldots \ldots \ldots \ldots \ldots$ iii

TABLE OF CONTENTS ............................................... iv



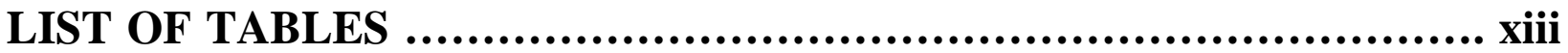

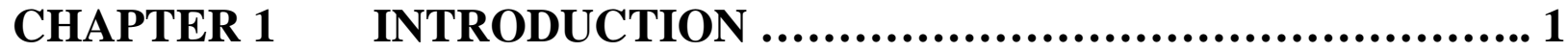

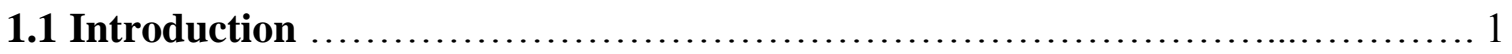

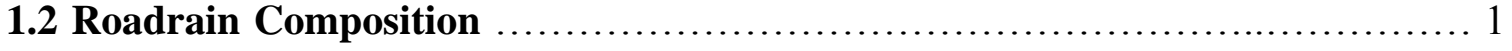

1.3 Objectives and Scope of Research ...................................... 2

CHAPTER 2 LITERATURE REVIEW AND BACKGROUND

INVESTIGATION ......................................... 4

2.1 General Statements on the Use of Concrete Pavements ......................... 4

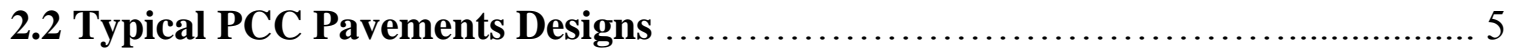

2.2.1 General PCC Pavement Cross-Sections .............................. 6

2.2.2 PCC Slab Support Layers ........................................... 6

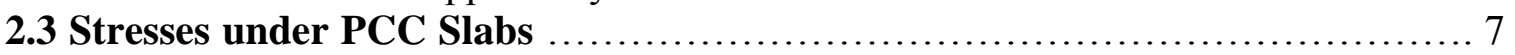

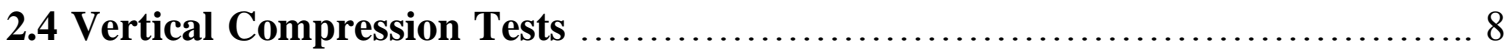

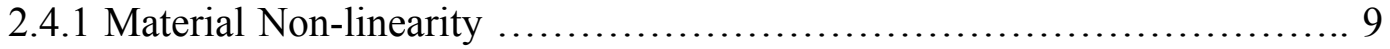

2.4.2 Multi-layer Uniaxial Compression Tests ........................... 10

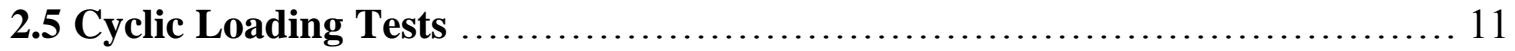

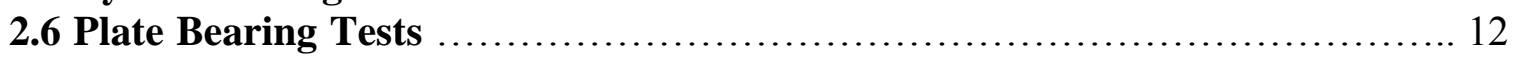

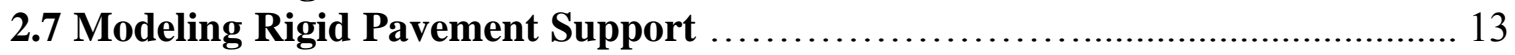

2.7.1 Theoretical Models for Settlement of Soils ........................... 14

2.7.2 AASHTO and PCA Methods ........................................... 16

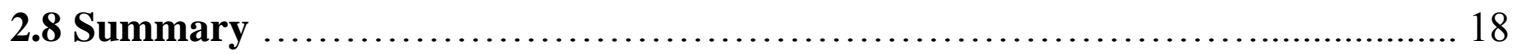

CHAPTER $3 \quad$ VERTICAL COMPRESSION TESTS .................... 32



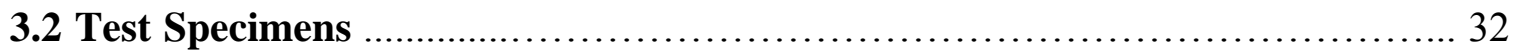

3.2.1 Concrete Mix Designs .......................................... 32

3.2.2 Specimen Preparation ................................................ 33

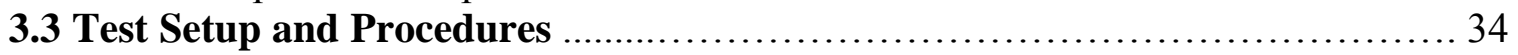

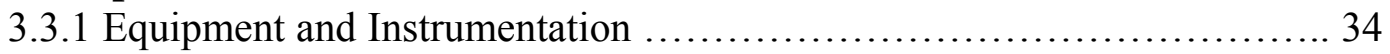

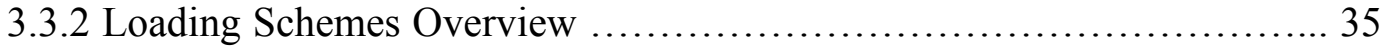

3.3.3 Procedure for Load-Release Tests ................................ 36 
3.3.4 Procedure for Repeated Load-Release Tests ........................ 36

3.3.5 Procedure for Sustained Loading Tests .............................. 37

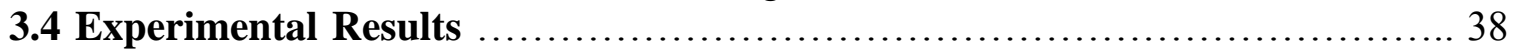

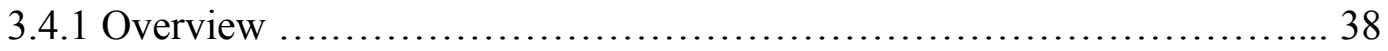

3.4.2 Load-Release Test Results ........................................ 38

3.4.3 Repetitive Load-Release Test Results: Loading Curves .................... 39

3.4.4 Repetitive Load-Release Test Results: Unloading Curves ............... 41

3.4.5 Sustained Loading Results ......................................... 42

3.4.6 Summary and Analysis of Results ................................ 43

CHAPTER 4 CYCLIC FATIGUE TESTS ............................69

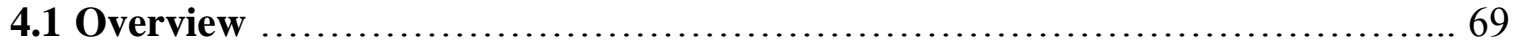

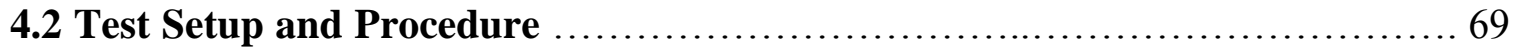

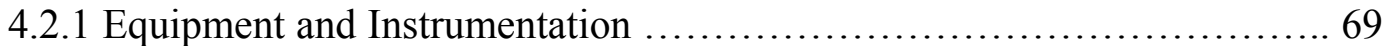

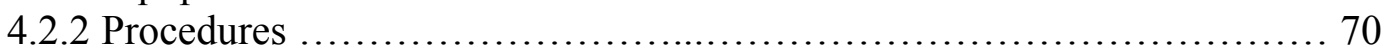

4.3 Experimental Results .............................................. 70

CHAPTER 5 PLATE BEARING TESTS ................................ 83

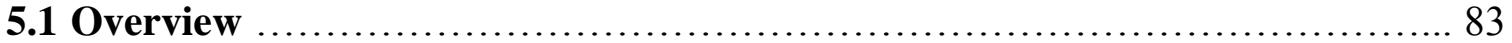

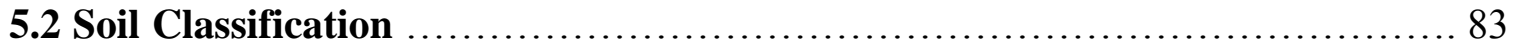

5.2.1 Soil Classification Tests ........................................ 83

5.2 .2 Initial Soil Preparation .......................................... 84

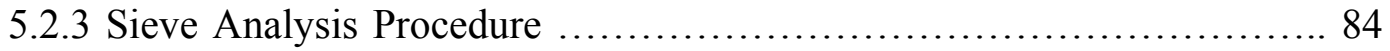

5.2.4 Plastic Limit Test Procedure ...................................... 84

5.2.5 Liquid Limit Test Procedure .......................................... 85

5.2.6 Direct Shear Test Procedure ....................................... 86

5.2.7 Soil Classification Results ......................................... 86

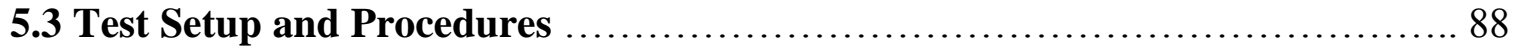

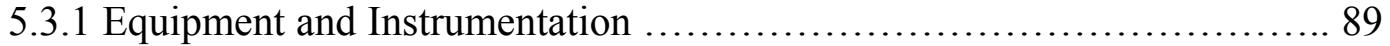

5.3.2 Subbase Preparation ................................................. 90

5.3.3 Non-Repetitive Static Plate Test Procedure ............................ 90

5.3.4 Repetitive Static Plate Test Procedure ............................... 91



5.4.1 Non-Repetitive Static Plate Bearing Test Data: Loading Curves ......... 92

5.4.2 Repetitive Static Plate Bearing Test Data: Loading Curves ................ 93

5.4.3 Comparison of Loading Curves from Plate Bearing Tests ............... 95

5.4.4 All Static Plate Bearing Test Data: Unloading Curves .................. 96

\section{CHAPTER $6 \quad$ PREDICTION OF SUPPORT STIFFNESS ............. 129}

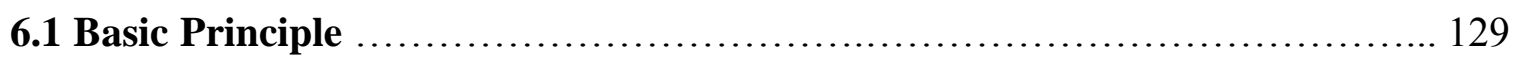

6.2 General Procedure ..................................................... 130

6.3 FE Analyses for Determining Soil Properties ............................. 131

6.3.1 General FEM Characteristics .................................................. 131

6.3.2 Material Properties Used for the Analyses ........................... 132

6.3.3 Results from Property Verification Models .......................... 133 
6.4 Sample Theoretical Calculations ........................................ 134

6.4.1 Theoretical Methodology and Results ............................... 134

6.4.2 Comparison of Theoretical and FE Results ........................... 135

6.4.3 Comparison of Theoretical to Experimental Results ..................... 136

CHAPTER 7 CONCLUSIONS AND RECOMMENDATIONS .......... 148

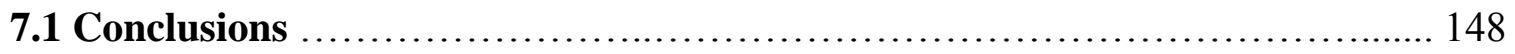

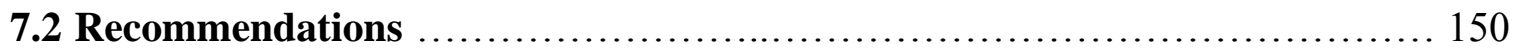

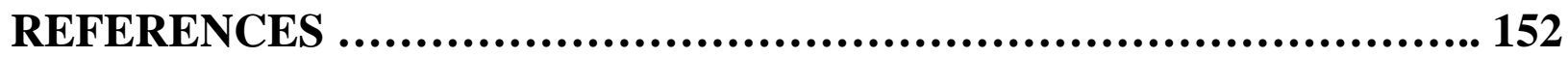

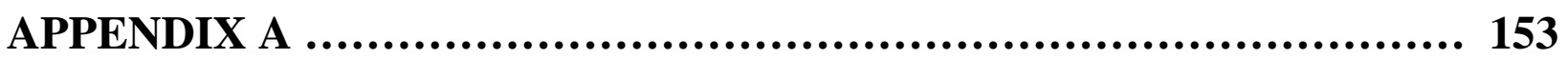

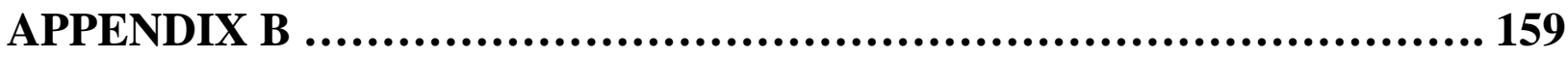




\section{LIST OF FIGURES}

Figure 1.1: Close-up of Roadrain Sample with Geonet Core Exposed

Figure 2.1: Various Types of PCC Pavements, as Defined by the Use of Saw-Cut Joints and Steel Reinforcement. (a) Jointed Plain Concrete Pavement, or JPCP, (b) dowelled JPCP, (c) Jointed Reinforced Concrete Pavement, or JRCP, and (d) Continuously Reinforced Concrete Pavement, or CRCP.

Figure 2.2: Typical Cross-Section of a Dowelled JPCP Saw-Cut Joint

Figure 2.3: Some Pavement Support Layers Used for Various Highway Constructions in (a), (b) South-western Pennsylvania, and (c) Central West Virginia.

Figure 2.4: Exploded View of the Material Layers Used for the Finite Element Analysis Described in Section 2.3

Figure 2.5: Schematic Diagram of the Locations of 5kip Point Loads Used for FE Modeling to Simulate a Dual-Tandem Truck Axle Configuration (Top View of Concrete Slab).

Figure 2.6: Maximum Principal Stresses obtained from the Finite Element Modeling Described in Section 2.3: (a) Shown with Slab, (b) Shown with Slab Removed to Expose Subbase Surface.

Figure 2.7: Maximum Principal Stresses at Selected Cross-Sections from FEM Results: (a) Locations of Cross-Sections, (b) Stresses at Section A-A, and (b) Stresses at Section B-B.

Figure 2.8: Examples of Non-linear Stress-Strain Relationships Exhibited by Concrete: (a) General Behavior, (b) Some Typical Linear Approximations, and (c) Laboratory Data and Stiffness Calculations from the 28-Day Compression Test of a 6in.x12in. Concrete Cylinder.

Figure 2.9: Results of Preliminary Compression Tests Done in Phase I using Two Roadrain ["Tendrain"] Samples Bonded to Concrete Cylinders

Figure 2.10: Simplified Model of a Uniaxial Compression Load Acting on a Two-Layered Specimen

Figure 2.11: Initial Strain Behavior of a Material Which Undergoes Plastic Behavior under Repeated Loading.

Figure 2.12: Picture of Field Plate Bearing Test Setup as Conducted in the Field

Figure 2.13: Basic Definitions of Typical Models Used for Determining the Stress Increase at Point "A" below a Uniformly Loaded Flexible Circular Area

Figure 2.14: AASHTO Design Chart for Estimating Composite Modulus of Subgrade Reaction Assuming a Semi-Infinite Subgrade Depth

Figure 3.1: (a) Schematic Diagram, and (b) Picture of Vertical Compression Test Specimens.

Figure 3.2: (a) Schematic Diagram, and (b) Picture of Batch \#1 Vertical Compression Test Specimens and Instrumentation

Figure 3.3: (a) Schematic Diagram, and (b) Picture of Batch \#2Vertical Compression Test Specimens and Instrumentation 
Figure 3.4: Sample Picture. Batch \#1 Vertical Compressive Test Setup

Figure 3.5: Loading and Unloading Curves for Roadrain from Load-Release Tests: (a) All $w / c=0.4$, (b) All $w / c=0.57$.

Figure 3.6: Stress-Strain Curves from Load-Release Testing of All Batch \#1 Specimens

Figure 3.7: Characteristic Curve for Roadrain Based on Load Portion of Load-Release Results

Figure 3.8: Sample Stress-Strain Data Collected from the Repetitive Load-Release Tests (Data from Cylinder C2WC04 \#1)

Figure 3.9: Stress-Strain Diagrams of the Loading portions of Repetitive Load-Release Tests of Cylinders (a) C2WC04 \#1, (b) C2WC04 \#1b, (c) C2WC04 \#3, (d) C2WC057 \#2, and (e) C2WC057 \#3.

Figure 3.10: (a) Comparison of Final Loadings of Each Magnitude for All Repetitive LoadRelease Test Specimens; (b) Best-Fit Characteristic Curve from Final Loadings

Figure 3.11: Sample Stress-Strain Curves Collected from the Unloading Portion of the Repetitive Load-Release Tests (Data from Cylinder C2WC04 \#1)

Figure 3.12: Rebound Stress-Strain Data from Release Portions of All Repetitive Load-Release Test Specimens; Unloading from Loads of (a) $250 \mathrm{lbs}$, (b) $500 \mathrm{lbs}$, (c) $1000 \mathrm{lbs,} \mathrm{(d)} 1500 \mathrm{lbs}$, (e) $2500 \mathrm{lbs}$, (f) $3000 \mathrm{lbs}$, and (g) $4000 \mathrm{lbs}$

Figure 3.13: Comparison of Trendlines of Rebound Deflections from Unloading of Various Load Levels; All Repetitive Load-Release Tests

Figure 3.14: Comparison of the Slopes of the Trendlines of Rebound Deflections from Unloading of Various Load Levels; All Repetitive Load-Release Tests

Figure 3.15: Stress-Strain Data Collected from the Sustained Loading Tests Conducted on Specimen (a) C2WC04 \#2, and (b) C2WC057 \#1

Figure 3.16: Trendlines Derived from the Sustained Loading Tests Conducted on Specimen (a) $\mathrm{C} 2 \mathrm{WC} 04$ \#2, and (b) C2WC057 \#1

Figure 3.17: Comparison of Trendlines Derived from the Final Sustained Loading Tests Conducted on Specimens C2WC04 \#2 and C2WC057 \#1

Figure 3.18: Comparison of Characteristic Stress-Strain Curves for Loads up to 100 psi

Figure 3.19: Comparison of Characteristic Stress-Strain Curves for Loads up to 2,000 psi

Figure 4.1: Overview of Cyclic Fatigue Testing Setup

Figure 4.2: Cyclic Fatigue Test Specimen, Instrumentation, and Loading Block

Figure 4.3: Unloading Data Obtained at the Conclusion of the Fatigue Test of Specimen 2

Figure 4.4: Average Displacement vs. Time for Cyclic Fatigue Test of Specimen 2 after 20,000 Cycles. Loading Range: 0 to 20,000 lbs; Loading Frequency: $0.25 \mathrm{~Hz}$

Figure 4.5: Cumulative Plastic Strain vs. Number of Cycles. Both Samples Subjected to an Applied Cyclic Loading of 35 psi 
Figure 4.6: Elastic Strain vs. Number of Cycles. Both Samples Subjected to an Applied Cyclic Loading of 35 psi.

Figure 4.7: Roadrain Resilient Modulus vs. Number of Cycles. Both Samples Subjected to an Applied Cyclic Loading of 35 psi.

Figure 5.1: Sieves Used for Sieve Analysis of Excavated Soil

Figure 5.2: Liquid Limit Device (Left) and Grooving Tool (Right) Used for Liquid Limit Test, and Approximate Orientation of Groove as Formed in Sample Using the Grooving Tool

Figure 5.3: Direct Shear Test Setup Used for Soil Classification for Excavated Soil of Plate Bearing Tests

Figure 5.4: Grain Sizes Obtained from the Sieve Analysis of Excavated Soil Used as a Subbase for Plate Bearing Tests

Figure 5.5: Flow Curve for Liquid Limit Determination of Excavated Soil Used as a Subbase for Plate Bearing Tests

Figure 5.6: Shear Stress vs. Shear Strain Curve from Direct Shear Testing of Excavated Soil Used as a Subbase for Plate Bearing Tests

Figure 5.7: Shear Stress (@ 10\% Strain) vs. Normal Stress from Direct Shear Testing of Excavated Soil Used as a Subbase for Plate Bearing Tests

Figure 5.8: Approximate Interrelationships of Soil Classifications and Bearing Values

Figure 5.9: Overall View of Plate Bearing Test Setup

Figure 5.10: Flow Chart for Subbase Preparation and Testing History of Non-Repetitive Tests Utilizing \#57 Limestone Aggregate Subbases

Figure 5.11: Flow Chart for Subbase Preparation and Testing History of Non-Repetitive Tests Utilizing A-2-4 Soil Subbases

Figure 5.12: Flow Chart for Subbase Preparation and Testing History of Repetitive Tests Utilizing \#57 Limestone Aggregate Subbases

Figure 5.13: Flow Chart for Subbase Preparation and Testing History of Repetitive Tests Utilizing A-2-4 Subbases

Figure 5.14: Picture of Sample, Loading Plates and Instrumentation Setup for Test of an A-2-4 Soil Subbase (a) without Roadrain, and (b) with Roadrain (shown w/o Load Cell)

Figure 5.15: Schematic Overhead View of Instrumentation Placement

Figure 5.16: Schematic Side View of Instrumentation Placement

Figure 5.17: Plate Tamper Used for Subbase Compaction, Plate Bearing Tests

Figure 5.18: Sample Load-Deflection Curve from Non-Repetitive Plate Bearing Tests

Figure 5.19: Stress-Deflection Curves from All Non-Repetitive Plate Bearing Tests Using \#57 Limestone Aggregate Subbases

Figure 5.20: Stress-Deflection Curves from All Non-Repetitive Plate Bearing Tests Using A-24 Soil Subbases 
Figure 5.21: Stress-Deflection Curves from All Trials of the Non-Repetitive Plate Bearing Tests of a Recompacted \#57 Limestone Aggregate Subbase plus a Roadrain Layer

Figure 5.22: Stress-Deflection Trendlines from Final Trials of All Non-Repetitive Plate Bearing Tests Using \#57 Limestone Aggregate Subbases

Figure 5.23: Stress-Deflection Trendlines from Final Trials of All Non-Repetitive Plate Bearing Tests Using A-2-4 Soil Subbases

Figure 5.24: Load-Displacement History of \#57 Limestone Aggregate Subbase (Without Roadrain Layer) When Exposed to A Repetitive Static Plate Loading of 2,100 lbs.

Figure 5.25: Normalized Stress-Displacement History of an A-2-4 Soil Subbase plus Roadrain Layer When Exposed to A Repetitive Static Plate Loading of 16,000 lbs: (a) Initial Curve, and (b) Normalized Curve after Removal of Plastic Deformation.

Figure 5.26: Characteristic Curves Obtained from Repetitive Plate Bearing Tests of All Support Systems Using \#57 Limestone Aggregate Subbases Subjected to Multiple "Medium” Load and "Large" Load Applications.

Figure 5.27: Characteristic Curves Obtained from Repetitive Plate Bearing Tests of All Support Systems Using A-2-4 Soil Subbases Subjected to Multiple "Medium" Load and "Large" Load Applications

Figure 5.28: Characteristic Curves Obtained from Repetitive Plate Bearing Tests of All Support Systems Subjected to Multiple "Small" Load Applications

Figure 5.29: Comparison of All Characteristic Plate Bearing Test Loading Curves Obtained with \#57 Limestone Aggregate Subbase

Figure 5.30: Comparison of All Characteristic Plate Bearing Test Loading Curves Obtained with A-2-4 Soil Subbase

Figure 5.31: Sample of Unloading Curves from the Static Plate Bearing Tests; Obtained from the Non-Repetitive Loading of a \#57 Limestone Aggregate Subbase plus a Roadrain Layer.

Figure 6.1: Picture of Distinct Imprint on Bottom Side of Roadrain Layer at the Conclusion of a Plate Bearing Test with A-2-4 Soil Subbase

Figure 6.2: Exploded View of Component Geometries used for Axisymmetric FE Modeling of Non-Repetitive Plate Bearing Tests of Soil Subbases without Roadrain

Figure 6.3: Comparisons of FEA Results to Experimental Data from Non-Repetitive Plate Bearing Tests of Soil Subbases without Roadrain. (a) \#57 Limestone Subbase, and (b) A-2-4 Soil Subbase.

Figure 6.4: Locations of Deflection Readings for FE Analysis of Plate Bearing Tests Figure 6.5: Sample Stress Distribution: Load=9250lbs. on A-2-4 Soil Subbase Alone Figure 6.6: Egorov's Constants Used for Theoretical Calculations of Elastic Settlement

Figure 6.7: Calculated Theoretical Composite Stiffnesses Assuming Load Range from 0psi to Peak as Indicated. (a) \#57 Limestone and (b) A-2-4 Soil Subbases. 
Figure 6.8: Comparisons of Theoretical Deflections to FEA Results from Simulation of NonRepetitive Plate Bearing Tests of Soil Subbases without Roadrain. (a) \#57 Limestone Subbase, and (b) A-2-4 Soil Subbase.

Figure 6.9: Exploded View of Component Geometries used for Axisymmetric FE Modeling of Non-Repetitive Plate Bearing Tests of A-2-4 Soil Subbase with Roadrain

Figure 6.10: Comparisons of Theoretical Deflections with FEA Displacements at the Center of the Largest Loading Plate for Non-Repetitive Plate Bearing Tests of A-2-4 Subbases with Roadrain Using Two Different Roadrain Stiffnesses

Figure 6.11: Comparisons of Theoretical Deflections with Experimental Displacements at the Center of the Largest Loading Plate for Non-Repetitive Plate Bearing Tests of \#57 Limestone Subbases with and without Roadrain

Figure 6.12: Comparisons of Theoretical Deflections with Experimental Displacements at the Center of the Largest Loading Plate for Non-Repetitive Plate Bearing Tests of A-2-4 Soil Subbases with and without Roadrain.

Figure A.1: Raw Data from Repetitive Load-Release Vertical Compression Test of C2WC04 \#1

Figure A.2: Raw Data from Repetitive Load-Release Vertical Compression Test of C2WC04 $\# 1 \mathrm{~b}$

Figure A.3: Raw Data from Repetitive Load-Release Vertical Compression Test of C2WC04 \#3

Figure A.4: Raw Data from Repetitive Load-Release Vertical Compression Test of C2WC057 \#2

Figure A.5: Raw Data from Repetitive Load-Release Vertical Compression Test of C2WC057 \#3

Figure B.1: Raw Data Collected for the Repetitive Static Plate Bearing Tests of \#57 Limestone Subbases Both with and without a Roadrain Layer. Tested at (a) Small, (b) Medium, and (c) Large Load Magnitudes

Figure B.2: Raw Data Collected for the Repetitive Static Plate Bearing Tests of A-2-4 Soil Subbases Both with and without a Roadrain Layer. Tested at (a) Small, (b) Medium, and (c) Large Load Magnitudes

Figure B.3: Rebound Curves for all Non-Repetitive Static Plate Bearing Tests Using an A-2-4 Soil Subbase: (a) Subbase Alone, (b) Subbase Plus Roadrain, and (c) Recompacted Subbase Plus Roadrain.

Figure B.4: Rebound Curves for all Non-Repetitive Static Plate Bearing Tests Using an A-2-4 Soil Subbase: (a) Subbase Alone, (b) Subbase Plus Roadrain, and (c) Recompacted Subbase Plus Roadrain.

Figure B.5: Rebound Curves for Mid-Load Repetitive Static Plate Bearing Tests Using \#57 Limestone Aggregate Subbase: (a) Subbase Alone, (b) Subbase Plus Roadrain

Figure B.6: Rebound Curves for Large-Load Repetitive Static Plate Bearing Tests Using \#57 Limestone Aggregate Subbase: (a) Subbase Alone, (b) Subbase Plus Roadrain 
Figure B.7: Rebound Curves for Mid-Load Repetitive Static Plate Bearing Tests Using A-2-4 Soil Subbase: (a) Subbase Alone, (b) Subbase Plus Roadrain

Figure B.8: Rebound Curves for Large-Load Repetitive Static Plate Bearing Tests Using A-2-4 Soil Subbase: (a) Subbase Alone, (b) Subbase Plus Roadrain 


\section{LIST OF TABLES}

Table 2.1: Material Properties and Element Sizes Used for FEM Representation for Determining Typical Range of Non-Dowelled JPCP Subbase Stresses under Service Loads

Table 2.2: Variations of Egorov's Constants for Calculating Settlement below the Center of a Circular Plate

Table 2.3: PCA Design [Composite] $k$-values When Considering the Effects of (a) Untreated Subbases, and (b) Cement-Treated Subbases of Varying Thicknesses

Table 3.1: Mix Designs for the Two Concrete Mixes Used in Vertical Compression Test Specimens

Table 3.2: Loading Scenario for Each Vertical Compression Test Specimen

Table 3.3: Target Loads for Each Load-Release Compressive Test Specimen

Table 3.4: Load Patterns for Repetitive Load-Release Tests

Table 3.5: Load Patterns for Sustained Loading Tests

Table 3.6: Summary of Stress-Strain Relationships for Roadrain as Observed in Vertical Compression Tests

Table 4.1: Rate(s) of Loading during Readings of Deflection Data and Number of Cycles Completed before the Reading for Two Fatigue Tests

Table 4.2: Changes of Material Properties of Roadrain at Selected Points as Observed During the Cyclic Fatigue Tests. (a) Accumulated Plastic Strain, and (b) Resilient Modulus

Table 4.3: Unloading Stiffnesses and Moduli as Observed at the Conclusion of Each Respective Fatigue Test

Table 5.1: Suggested Values for Poisson's Ratio of Various Types of Soils

Table 5.2: Loading Scenarios Used for All Plate Bearing Tests Using \#57 Limestone Subbase

Table 5.3: Loading Scenarios Used for All Plate Bearing Tests Using A-2-4 Soil Subbase

Table 5.4: Characteristic Curves Obtained from Multiple Repetitive and Non-Repetitive Static Plate Bearing Tests Conducted on \#57 Limestone Subbases

Table 5.5: Characteristic Curves Obtained from Multiple Repetitive and Non-Repetitive Static Plate Bearing Tests Conducted on A-2-4 Soil Subbases

Table 6.1: Final Material Properties Used in the FE Analyses Representing the Non-Repetitive Static Plate Loadings of Subbases without Roadrain

Table 6.2: Comparison of Central and Outer Deflections as Obtained from the FE Analyses of Subbases without Roadrain

Table 6.3: Calculated Elastic Settlement [in Inches] at Center of Loading Plate for Subbases Systems with and without Roadrain: (a) \#57 Limestone Subbase, and (b) A-2-4 Soil Subbase

Table 6.4: Comparison of Central and Outer Deflections as Obtained from the FE Analyses of A-2-4 Soil Subbases with Roadrain 


\section{CHAPTER 1 INTRODUCTION}

\subsection{Introduction}

Drainage is a crucial component of a long-lasting pavement structure in any type of pavement. Proper drainage could also aide in providing uniform support of the pavement by reducing the influences from shrinkage and swelling of expansive soils in addition to resulting in a stiffer subgrade. Typically, a granular subbase, which is often stabilized with small amounts of asphalt or Portland cement, is used to act as the drainage layer for a pavement system. Roadrain $^{\mathrm{TM}}$, a geocomposite drainage layer produced by Tenax Corporation, is designed for the purpose of providing roadway drainage, and can possibly replace the typical drainage layer for concrete pavement systems. However, since Roadrain is a composite material and therefore behaves different from granular materials, many of its characteristics need to be examined in order to design Portland Cement Concrete (PCC) pavements. Phase I of this study investigated the frictional effects, bond strength, and effects on concrete shrinkage of a Roadrain layer when incorporated directly below the PCC pavement. This phase, Phase II, focuses on the vertical loads to which the highway will be subjected, and will give highway engineers a means for performing designs which incorporate Roadrain as a drainage layer, particularly with respect to slab thickness designs.

\subsection{Roadrain Composition}

Roadrain Roadway Drainage Geocomposite is composed of three layers: a geonet core with thermally-bonded geotextiles attached to both the top and the bottom. The geonet core is comprised of a multi-directional plastic grid which allows water to flow through. Although the geotextiles can be composed of either continuous fibers or short-strand fibers, only those with continuous fibers were used for this set of experiments; the difference in vertical stiffnesses between Roadrain samples using the two types of geotextiles would be expected to be negligible since load distribution for the geocomposite is primarily attributed to the Geonet core. A picture of a Roadrain sample is shown in Figure 1.1 with the top fiber layer receded as to expose the Geonet core. 


\subsection{Objectives and Scope of Research}

The main objective of this research was to give highway design engineers the tools to account for the use of Roadrain in their pavement thickness designs for concrete pavements. To do this, it was first necessary to obtain sufficient data characterizing the stiffness of Roadrain when it alone is subjected to vertical loads. Ideally, all types of loads to which the Roadrain would be subjected when incorporated into the roadway structure would be considered; those chosen for this study aimed at observing short-term creep effects and the development of plastic deformation after the application of numerous loads. Both vertical static compression testing and cyclic testing were conducted.

Once the stiffness characteristics of Roadrain were obtained, laboratory-simulated plate bearing tests were used to correlate the stiffnesses of the Roadrain and of two different types of subbases to the overall stiffness of both layers; this overall stiffness is the property needed for the thickness design for PCC pavements. Theoretically-derived relationships are given and verified according to the data obtained from the plate bearing tests.

A literature review was conducted for all phases of this project, and its results are summarized in Chapter 2; Chapters 3 and 4 present the vertical compression testing and cyclic loading tests, respectively; Chapter 5 describes the Plate Bearing Tests that were conducted; Chapter 6 presents the correlations between Roadrain stiffness and overall stiffness, as well as a comparison of the correlations to the experimental Plate Bearing Test data. Chapter 7 gives conclusions and recommendations for using Roadrain beneath PCC pavements. 


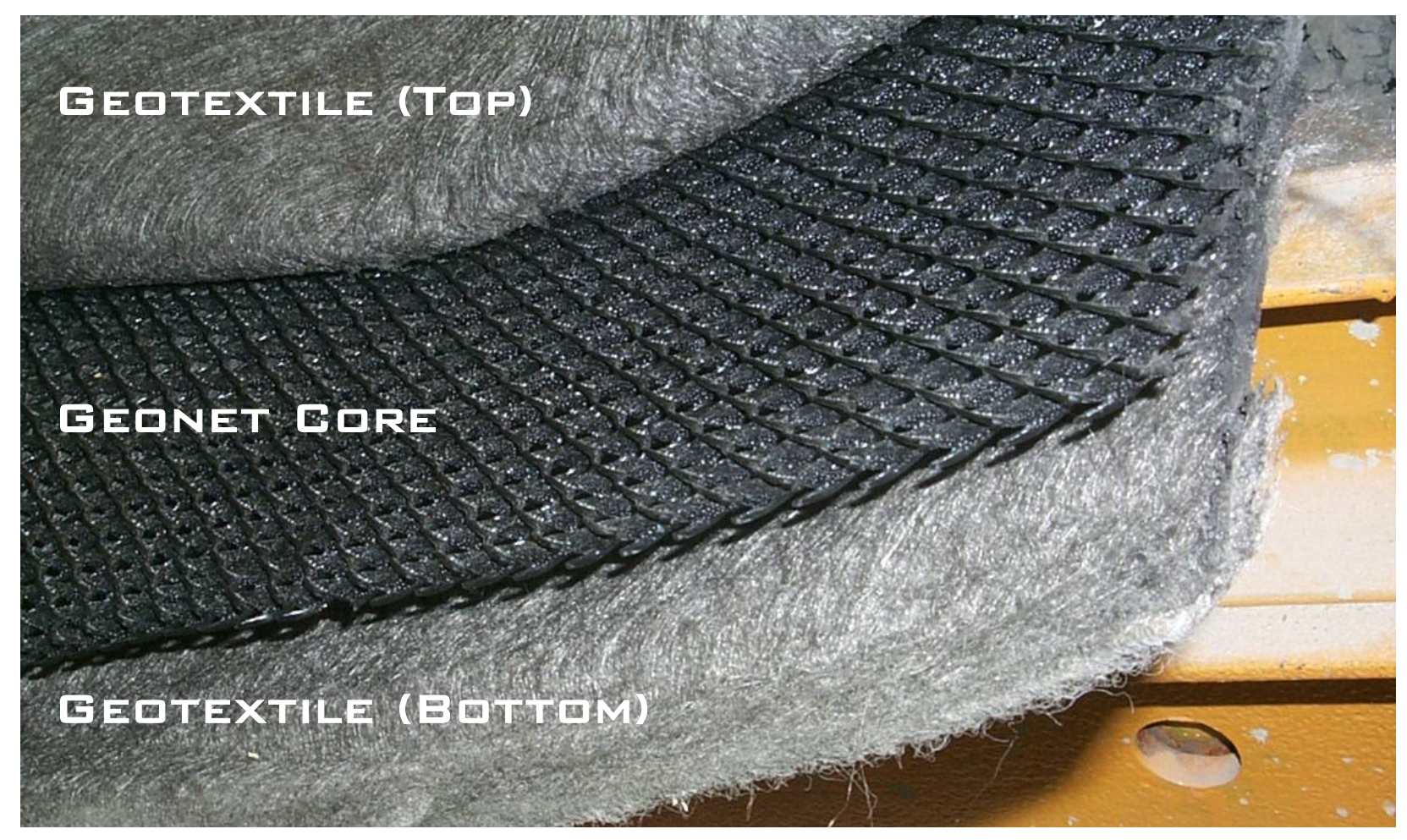

Figure 1.1: Close-up of Roadrain Sample with Geonet Core Exposed 


\section{CHAPTER 2}

\section{LITERATURE REVIEW AND BACKGROUND INVESTIGATION}

\subsection{General Statements on the Use of Concrete Pavements}

PCC has long since been used by the civil engineering community as a cornerstone of numerous structural designs. In particular, PCC pavements have played a large roll in the development of the United States infrastructure, comprising approximately $30 \%$ of interstate highways (Consedine). While there are numerous types of PCC pavement designs, some of which are shown in Figures 2.1(a) through 2.1(d), among the most abundant are Jointed Plain Concrete Pavements (JPCPs) [as depicted by Figures 2.1(a) and 2.1(b), although the majority of this category are of type (b)], which do not contain reinforcement for the purpose of controlling thermal and shrinkage cracks, and Continuously Reinforced Concrete Pavements (CRCPs), which use reinforcement throughout for this purpose [see Figure 2.1(d)]. Although the use of JPCP pavements may currently be declining in some parts of the country, partially due to the SUPERPAV design method which gives practical guidelines for the improvement upon the reliability of Asphalt Concrete (AC) pavements, case studies and recent innovations throughout the world have shown that concrete pavements have many desirable performance characteristics which should help pavement design and construction engineers to promote concrete use in future pavement constructions.

For example, economic advantages from using $\mathrm{PCC}$, as opposed to $\mathrm{AC}$, for pavement structures, especially in colder climates, come from longer service lives and reduction in maintenance costs, while the rigidity of concrete pavements gives motorists the advantage of a reduced risk of hydroplaning, better reflectivity improves night vision, and decreased roadway deflections result in better fuel economy for large trucks (Consedine). For these reasons, plus the reduction in the formation of potholes in rigid PCC pavements, and the increased resistance of a well-designed concrete mix to deterioration as a result of roadway deicing salts, PCC pavements are currently being considered as a possible solution to many of the current cold-weather problems in Canada, even though this idea has not been implemented on a grand scale yet, as Canadian provinces currently contain only small percentages $(<4 \%)$ of PCC pavements (Consedine). 
A testament to the longevity of concrete pavements comes from the state of Illinois. More than 2,650 two-lane miles $(4,267 \mathrm{~km})$ of CRCPs have been build on the Interstate system since the mid-1950s, and a study of this Illinois CRCP shows that regardless of the thickness or steel content used in the original design, all CRCPs exceeded their expected design lives and most have carried more traffic than that for which they were designed (Gharaibeh). In addition, many European nations are turning to concrete pavements, mostly JPCPs and CRCPs, in order to provide relatively service-free pavements which are designed for longer service lives and subjected to higher allowable single-axle loads than in the U.S. [30 to 40 years versus a typical 20 years, and 22.0 to 28.6 kips versus a typical 20 kips, respectively] (FHWA).

A 1992 report developed in a collaboration with United States highway and material research agencies, including AASHTO and FHWA, describes many of the current European concrete pavement design and construction practices as innovative and well-conceived, producing durable slabs on well-drained bases, and in turn recommends that the United States should focus on the continuous improvement of our own concrete pavement design practices (FHWA). The report notes that the increases in service life or capacity of the European highways was not due to an increase in thickness, as thicknesses were similar to those used in the United States; this was attributed, at least in part, to the emphasis on the design of the pavement system as a whole, including subbase drainage, not just the thickness of the slab.

On the whole, Roadrain is potentially very beneficial to the overall structure of a PCC pavement system. Aside from the primary function of providing drainage, the Geotextile fabric layers would also act as filters which would prevent pumping of fine aggregates, thereby minimizing erosion and avoiding critical losses of roadbed support at the locations of joints. Also, the Roadrain would have a greater ability than the rigid PCC slab itself to deform and absorb any localized irregularities in subgrade support, thereby reducing the magnitudes of resulting slab stresses.

\subsection{Typical Designs for PCC Pavements}

As was mentioned in Section 2.1, the most common types of PCC pavements used for highway driving surfaces in the U.S. are Jointed Plain Concrete Pavements (JPCPs) and Continuously Reinforced Concrete Pavements (CRCPs). Both use different techniques to account for the fact that the concrete used for the pavement slab shrinks both immediately due to 
changes in ambient temperatures, and in the long-term over the course of its service life. Some of their most traditional components will be discussed briefly in this section.

\subsubsection{Typical PCC Pavement Cross-Sections}

JPCPs simply create saw-cut joints in the pavement at regular intervals (usually spaced $12 \mathrm{ft}$ to $15 \mathrm{ft}$, but sometimes up to 60 -ft spacings are used) which act to create stress concentrations in the slab as it shrinks, promoting cracking at those locations, meanwhile assuring a smoother roadway surface by controlling the geometry of the failure. To allow shear transfer at the joints of JPCPs, steel or FRP dowel bars are usually implemented along the length of the joints to provide some sort of continuity between adjacent PCC slabs. Typical sizes for a saw-cut joint can be seen in Figure 2.2.

CRCPs use a different technique to control cracking in PCC slabs; in this type of pavement, reinforcing bars, or rebars, which are typically made of steel or in some cases Fiber Reinforced Plastic (FRP) composite materials, act as temperature and shrinkage reinforcement. By controlling the amount of restraint to volume change provided by the reinforcement, in conjunction with the restraint provided by the subbase friction, the CRCP can be designed such that cracks form at desirable intervals, also resulting in acceptable crack widths.

\subsubsection{PCC Slab Support Layers}

PCC pavements are also often referred to as "rigid pavements," as opposed to the socalled "flexible pavements" which use Asphalt Concrete. The reason for this is mainly due to the fact that the thick slab layer composed of PCC experiences only very small deflections when subjected to traffic loads, transmitting only small stresses distributed over a large area to the underlying layers, whereas the $\mathrm{AC}$ slabs experience more localized deformations and rely more heavily upon the supporting layers to help carry the traffic loads. The result of this is that PCC slabs can be constructed on a variety of support systems with a wide range of stiffnesses and still perform adequately.

The simplest type of support system used for PCC pavements is that which utilizes the existing subgrade alone without the implementation of intermediate layers below the slab. The subgrade is typically compacted in preparation; this is done primarily to improve upon the strength and the uniformity of support provided. Most systems that are used for highways, 
however, do incorporate other layers into the design. The depths and compositions of the layers vary, but the many designs include a subbase and/or a drainage layer. The primary function of the subbase is to strengthen the overall support system and to provide uniformity of support; this layer is typically comprised of aggregate which may or may not be treated with either asphalt or PC for stabilization. Drainage layers help provide run-off of rain water; drainage layers are typically made of open-graded aggregates and may also be stabilized. Sometimes layers act in strengthening and providing drainage for a highway system. Other common elements that are often incorporated into highway systems include filter-fabric to detain fine aggregates from migrating into the upper layers of the highway system, and edge drains for drainage purposes. Some typical systems which have been used in West Virginia and southeastern Pennsylvania for the construction of JPCPs can be seen in Figures 2.3(a) through 2.3(c).

\subsection{Stresses under PCC Slabs}

In order to determine the approximate magnitudes of stresses to which a Roadrain layer would be subjected when used in the field as a part of a PCC pavement structure, a simplified Finite Element (FE) Analysis was done to simulated possible service-load conditions; the stress levels obtained would ultimately to act as guidelines for the laboratory experiments done throughout this investigation. The FE model used for this analysis was created using Algor Software. It was decided to use an un-doweled JPCP section, due to the simplicity of this type of pavement. Since this type of pavement lacks shear transfer devices (dowel rods or continuous reinforcing bars), the results of this FE analysis should give higher subbase stresses than stresses obtained from using a doweled JPCP or a CRCP highway. Load transfer effects from longitudinal joint ties were also neglected for this analysis.

The section used had dimensions of $15 \mathrm{ft}$ in length, $12 \mathrm{ft}$ in lane width, and a depth of 8 inches. It was supported by a 3-layer system, comprised of a 6-inch-deep subbase course and an 18-inch-deep compacted subgrade, both atop a dense liquid subgrade; all underlying layers had dimensions of $21 \mathrm{ft}$ in length and $18 \mathrm{ft}$ in width. Solid brick elements were used to create the concrete slab, the subbase and the compacted subgrade, and the nodes at the bottom of the subgrade were assumed to be fixed. For simplicity, all materials used in the model were assumed to exhibit linear-elastic behavior; also, the contact surfaces between the materials were assumed to be bonded. The material properties used for each type of material, along with their 
respective element sizes can be seen in Table 2.1., and a diagram of the layer configuration can be seen in Figure 2.4.

The loading applied to the slab had the geometry of a typical dual-wheel, tandem truck

axle. A load of 5,0001bs was applied to each wheel, totaling 40,0001bs for the axle, or the equivalent weight of a rear axle of an AASHTO-designated H-24 or HS-24 tractor-trailer live load. The contact area was reduced to a point load for each wheel. The axle was placed as close to the corner of the slab as possible in order to obtain the highest localized stress values (PCA, 1984). Schematic diagrams of the axle geometry and the wheel load placements can be seen in Figure 2.5.

The results of the subbase stress investigation can be seen in Figures 2.6 and 2.7. In the first of these figures, 2.6(a) is simply an overview of the maximum principal stresses encountered at the outer surface of the pavement system; Figure 2.6(b) shows the same perspective as Figure 2.6(a), except with the slab removed as to expose the stresses at the top of the subbase layer. It can be noted from these figures that, as to be expected, the stresses experienced by the slab are drastically greater than those in the supporting layers when the pavement system is subjected to vertical forces alone. This idea is accentuated by Figures 2.7(b) and (c), which show cross-sectional views of the pavement system when cut laterally at the dual locations of the wheel loads, as illustrated in Figure 2.7(a). From these figures, it can be seen that a typical stress for a sub-base for a non-dowelled JPCP can reach levels on the order of magnitude around 70 psi. Again, subbases for dowelled JPCPs and CRCPs should experience much smaller stress levels, since the shear transfer of these pavements would allow the axle load to be distributed to multiple slabs, and consequently over a larger area; ACPA describes these as being only about 3psi (ACPA, 1995), while Childs cites the subbase pressures due to a 9,0001b load exerted on the corner of an 8in. concrete slab as going up to about 18psi (Childs).

\subsection{Vertical Compressive Strength}

One of the simplest, and consequently one of the most common, means of determining mechanical properties of a material is by using a uniaxial loading test, which can be done by applying either compression or tension to a specimen. These tests give fundamental stress-strain relationships for a material, from which various engineering and behavioral properties can be established. For instance, from the applied stress versus longitudinal strain curve of a typical 
ductile, linear-elastic metal subjected to tension, such characteristics as its yield stress, fracture toughness, resilient modulus, and Young's modulus can be found. In addition, if lateral deflections are obtained the Poisson's ratio for the material can also be determined.

\subsubsection{Material Non-linearity}

The most useful material property from uniaxial tests, especially for linear-elastic materials, is the Young's modulus, or modulus of elasticity. Defined as the ratio of the applied stress to the resulting longitudinal strain (effective up to the point of yielding), this proves to be a valuable tool for predicting or modeling the behavior of actual structures, or components thereof, as they are exposed to various loadings. Since most structural components are designed such that they do not reach the yield point under service loads, the modulus of elasticity provides a very reasonable representation of the stress-strain relationship for linear-elastic materials in this region.

Non-linear stress-strain relationships, however, are possessed by many materials, and are sometimes problematic when attempting to establish a simplified "modulus-like" depiction of the material's behavior. In many analyses, especially for hand calculations, it is desirable for the sake of simplicity to assume a linear relationship that is, for the most part, representative of the actual non-linear material behavior. However, for a given non-linear stress-strain relationship the assumed modulus could vary greatly depending upon the values selected for its calculation.

An example of a common non-linear stress-strain relationship under uniaxial loading is the curve obtained from the compressive loading of concrete (Mindess and Young). As shown in Figure 2.8(a), concrete undergoes substantial creep deformation as the magnitude of the compressive load increases. It can also be seen that non-linear behavior is evident even under small loads, so no linear region is present for the calculation of a Young's modulus value. However, many different moduli may be ascertained from the same data set by calculating the slopes of various representative lines. Figure 2.8(a) also shows some of the representative lines whose slopes can be used to calculate the modulus of elasticity of the specimen. These moduli are generally termed "Secant modulus," "Chord modulus," etc. according to the nature of determination of the representative line, but all are intended as linear approximations of the material's stress-strain relationship over a predetermined stress range. It can be seen from Figure 2.8(b), a sample of laboratory data from an actual compression test of a 6"x12" concrete 
cylinder, that large variations in modulus values are present, depending upon the technique used for the linear approximation. Therefore one should consider the applicable stress range when attempting to assign the appropriate straight-line approximation to the material's non-linear behavior.

Roadrain has a different type of non-linearity than concrete. While concrete's tangential modulus decreases with increases in applied stress, Cook's preliminary compression tests of two samples show the opposite trend, indicating that Roadrain undergoes stiffening with increases of the applied loading magnitude. His load-displacement curves can be seen in Figure 2.9. His initial findings were built upon significantly throughout the laboratory portion of this study; the details of which will be outlined in Chapter 3.

\subsubsection{Multi-layer Uniaxial Compression Tests}

For the compression tests done in this phase, which will be discussed in Chapter 3, as well as the preliminary tests conducted by Cook in the first phase of this study, multiple-layered compression specimens were used which included both a 6 in $\mathrm{x} 12$ in concrete cylinder and a Roadrain layer bonded to one side. With this setup, it is necessary to devise a model which can be used to determine the contributions of both materials to the overall deflections that were measured. This was done using a simplified one-dimensional model using two springs to depict the uniaxial material behaviors, as shown in Figure 2.10. From this model, it can be seen that the overall deflection, which was measured in the laboratory, is equal to the sum of the deflections for each spring, or in our case:

$$
u_{\mathrm{C}}+u_{\mathrm{R}}=u_{\mathrm{C}+\mathrm{R}}
$$

where $u_{C}$ is the displacement of the concrete, $u_{R}$ is the displacement of the Roadrain, and $u_{C+R}$ is the overall displacement of the system. In addition, the stiffness contribution of the Roadrain layer for each stress range can be derived to have the following relationship:

$$
k_{R}=\frac{k_{C} \times k_{C+R}}{\left(k_{C}-k_{C+R}\right)}
$$

In this equation, $k_{R}$ represents the stiffness of the Roadrain, $k_{C}$ represents the stiffness of the concrete, and $k_{C+R}$ represents the composite stiffness of both the concrete and the Roadrain layer.

It can be seen from this relationship that as $k_{C}$ becomes very large, the value of $k_{R}$ approaches that of $k_{\mathrm{C}+\mathrm{R}}$. In our case, with a Young's modulus for concrete of approximately 
$4 \times 10^{6} \mathrm{psi}$, it can be seen from Cook's data that $k_{\mathrm{C}}>>k_{\mathrm{C}+\mathrm{R}}$, especially up to a load of around $40,000 \mathrm{lbs}$, or approximately 1,415psi. At that point it is believed that the Roadrain layer is either quickly approaching or already at a state of failure, but the stress levels are already well beyond the levels anticipated for Roadrain in the field, as discussed in Section 2.3. Therefore, in this stress range it will simply be assumed that $k_{\mathrm{R}} \approx k_{\mathrm{C}+\mathrm{R}}$ for the vertical compressive tests, and consequently that $u_{\mathrm{R}} \approx u_{\mathrm{C}+\mathrm{R}}$, and any errors in calculation due to these assumptions made for this particular application would be minute.

\subsection{Cyclic Loading Tests}

For rigid pavement design, it is specified in AASHTO design guidelines to determine a value $\mathrm{M}_{\mathrm{R}}$, which corresponds to resilient modulus of the roadbed soil (a.k.a. subgrade). This value, which is also used for layers in flexible pavement design (Asphalt Institute), is determined for unbound or relatively low stiffness materials using the resilient modulus test methods outlined in AASHTO T 274 (AASHTO). Similarly, the complex modulus test of ASTM D3497 gives a dynamic complex modulus for viscoelastic materials which is equivalent to the resilient modulus, both of which have been used for pavement design. Both tests use repeated loading to determine the corresponding modulus value, but the primary difference between the resilient modulus test and the complex modulus test is that the former includes a period of rest between loads during which no loading is applied, while the latter includes a continuous cycle of loading. The shapes of the loading curves for both tests are generally sinusoidal, but triangular and square curves have also been used for the resilient modulus test (Huang).

Under the repeated loading of these tests, a typical soil or visco-elastic specimen undergoes a strain pattern as indicated in Figure 2.11. It can be seen from this figure that even after the first load-release cycle some non-recoverable deformation is present in the material; this non-recoverable strain at any point represents the accumulated plastic strain. With the repetition of the loading, this inelastic element reduces when compared to the previous cycle, and becomes more consistent from cycle to cycle (PCA, 1992). Also, there is one portion of the strain which is recoverable upon release of the load; this is referred to as the elastic strain, $\varepsilon_{\mathrm{r}}$. At any point (but usually after about 200 cycles), the resilient modulus is defined as the ratio of the range of applied stress to the elastic strain at that point, or:

$$
\mathrm{M}_{\mathrm{R}}=\sigma_{\mathrm{d}} / \varepsilon_{\mathrm{r}}
$$


In this equation, $\sigma_{\mathrm{d}}$ is equal to the deviator stress, which is simply the axial stress in an unconfined test setup.

\subsection{Plate Bearing Tests}

The parameter used to represent the slab support from underlying layers in rigid pavement design is the modulus of subgrade reaction, $k$. Plate bearing tests are often done for insitu assessment of the subgrades in order to determine this value. For these tests, a load is applied to the center of a plate of a known diameter which has been placed on the subgrade, as shown in Figure 2.12. Both load and deflection readings are taken during the test. The modulus of subgrade reaction is simply defined as the ratio of the amount of stress necessary to cause a given deflection to the deflection itself, or:

$$
k=\frac{\operatorname{load}(p s i)}{\text { displacement }(\text { in })}
$$

PCA specifies the use of a displacement value of $0.05 \mathrm{in}$., and the corresponding load at $0.05 \mathrm{in}$. to define the modulus of subgrade reaction for slabs on ground (Farny), but for pavements the specifications vary by state or institution. For pavement design, PCA refers to the guidelines of ASTM D1196, the Non-Repetitive Static Plate Bearing Test, for determination of this $k$-value (PCA, 1984); this test procedure, however, does not specify an exact deflection at which to make this calculation (ASTM D1196). Some institutional and state pavement design guidelines, however, do specify the deflection at which this value was to be calculated; Florida, for example, also uses a deflection of $0.05 \mathrm{in}$. and the associated load level to define the modulus of subgrade reaction (FM 5-527).

ASTM gives guidelines for two types of static plate bearing tests for in-situ determination of a soil's $k$-value. As mentioned above, one type is the Non-Repetitive Static Plate Bearing test described in ASTM D1196; the other is the Repetitive Static Plate Bearing test described in ASTM D1195. For each test, load and deflection data are collected to characterize the loaddisplacement relationships of a soil, as well as the rebound characteristics for a given loading; the nature of the loading, however, is different for each test. Quite briefly, for the non-repetitive test the loading scenario is as follows: (1) a load of a small magnitude is applied and held until deflection virtually ceases, (2) without releasing that load, the magnitude of the load is increased and then sustained until deflection ceases again, (3) this sequence is repeated for at least six 
increasing load increments in order to obtain an accurate load-deflection curve for the loading, and (4) the load is released upon stabilization of the deflection under the highest sustained load. For the repetitive test, a load of a given magnitude is applied fully, sustained until deformation slows below a given rate, released, and after the rebound deformation has nearly ceased the load is re-applied. The same static load is applied and released multiple times consecutively for the same test, hence the name Repetitive Static plate bearing test. Both of these procedures were performed for the tests described in Chapter 5 and will be discussed more thoroughly at that time.

Although both the repetitive and non-repetitive plate bearing tests are generally acceptable means for determining the modulus of subgrade reaction, the two tests will usually give conflicting values for this property. The modulus obtained from the repetitive test is generally higher than that from the non-repetitive test, since for each successive repetition of the loading there is a reduction in the amount of plastic deformation which occurs (PCA, 1992).

\subsection{Modeling Rigid Pavement Support}

Many researchers have come to the same conclusion regarding the amount of support provided to rigid pavements and the corresponding slab stresses: the exact amount of support is not as crucial as the uniformity of the support when considering the longevity of the pavement (Childs, Farny, Grater, ACPA(1995), PCA(1984), Westergaard). This is because the forces from the rigid pavement are spread over such a large area that the corresponding subbase stresses are very small, as mentioned in Section 2.3. Consequently, slab stresses and deflections will be small regardless of the amount of support provided, so long as the support is uniform. Due to the low subbase and subgrade pressures, normal variations of slab support will not drastically affect the slab performance (Westergaard). Therefore, minor variations of this level of support from that assumed or calculated during the design process will not typically affect the necessary pavement thickness for a highway (PCA, 1984). Large slab stresses will result from abrupt localized changes in support, though. Nonetheless, for design purposes it is still desirable to get the best initial representation of slab support, especially when attempting to predict any affects that will come from incorporating an experimental layer such as Roadrain into the pavement structure. The remainder of this section will address methods for predicting the overall support provided by the system. Since both AASHTO and PCA designate the plate bearing tests as a 
means of determining the roadbed support, the numerical techniques will focus on predicting soil settlement when subjected to such a loading.

\subsubsection{Theoretical Models for Elastic Settlement of Soils}

The total settlement of a soil can be divided into three main components: (1) the elastic settlement, $S_{e}$, which takes place almost immediately after loading, (2) the primary consolidation settlement, $S_{C}$, and (3) the secondary consolidation settlement, $S_{S}$. Elastic settlement takes place without the expulsion of water or changes in the moisture content of the soil, primary consolidation settlement occurs due to the expulsion of water, and the secondary consolidation settlement occurs due to the rearrangement of the actual soil particles themselves. Altogether, the total settlement is as follows:

$$
S_{T}=S_{e}+S_{C}+S_{S}
$$

Since both the primary and secondary consolidation settlements are generally a long-term effects of sustained loading, the settlement experienced during plate bearing tests can be considered almost exclusively as elastic settlement. Therefore, the relationships given for settlement prediction in this section will concentrate on the elastic portion of settlement; in particular, the focus will be on the settlement of soil systems due to the uniform loading of a circular area, as is essentially the case of the plate bearing tests.

Having an accurate depiction of the pressure distribution is crucial for determining the settlement since the deformation at any point in a soil layer is a function of the pressure at that point, as is the case with all elastic materials. For this reason there have been many models developed which attempt to describe the pressure re-distribution of a semi-infinite soil layer due to an applied load (Yang). One common model for determining the vertical stress increase at any location below a uniformly loaded circular area is axisymmetrically-defined, as shown in Figure 2.13. Boussinesq defined the stress increase at any point, A, below the surface of a circularly loaded flexible area (with radius R) as being dependent upon: the magnitude of loading (per unit area), $q$, the distance below the surface of loading, $z$, the distance away from the center of loading, $r$, and the Poisson's ratio of the soil, $v$. 
If considering points directly below the center of the application of loading, the vertical stress increase, $\sigma_{z}$, can be written as:

$$
\sigma_{z}=q \cdot\left[1-\frac{z^{3}}{\left(R^{2}+z^{2}\right)^{3 / 2}}\right]
$$

and the stresses in the radial and tangential directions, $\sigma_{\mathrm{r}}$ and $\sigma_{\theta}$, respectively, can be written as:

$$
\sigma_{r}=\sigma_{\theta}=\frac{q}{2} \cdot\left[1+2 v-\frac{2(1+v) z}{\left(R^{2}+z^{2}\right)^{1 / 2}}+\frac{z^{3}}{\left(R^{2}+z^{2}\right)^{3 / 2}}\right]
$$

where $q, R, z$, and $v$ are as defined above. To expand upon these equations to include all points below a circularly loaded flexible area, values for relative increases in $\sigma_{z}, \sigma_{\theta}, \sigma_{r}$, and $\tau_{r z}$ which are dependent upon the loading, $q$, and upon the ratios $r / R$ and $z / R$ have been tabulated in detail by Ahlvin and Ulery (Das, 1999).

Using elastic theory, the vertical strain, $\varepsilon_{z}$, at any point due to a surface loading can be expressed as:

$$
\varepsilon_{z}=\frac{1}{E_{s}}\left[\sigma_{z}-v\left(\sigma_{r}+\sigma_{\theta}\right)\right]
$$

where $E_{s}$ is the elastic modulus of the soil. Subsequently, the elastic settlement of a semi-infinite elastic layer at that same point would be:

$$
\int_{z}^{\infty} \varepsilon_{z} d z=\int_{z}^{\infty} \frac{1}{E_{s}}\left[\sigma_{z}-v\left(\sigma_{r}+\sigma_{\theta}\right)\right] d z
$$

For a flexible plate supported by a semi-infinite layer, the following equations can be derived:

$$
S_{e}(\text { center }, \text { flexible })=\frac{2 R q}{E_{s}}\left(1-v^{2}\right) \text { and } S_{e}(\text { edge }, \text { flexible })=\frac{1.272 R q}{E_{s}}\left(1-v^{2}\right)
$$

The average settlement of a flexible plate would be approximately $0.85 *\left[S_{e}(\right.$ center, flexible $\left.)\right]$. If the plate is considered to be completely rigid, the settlement would be approximately:

$$
S_{e}(\text { center }, \text { rigid }) \approx 0.93 \cdot S_{e}(\text { average, flexible })=\frac{1.581 R q}{E_{s}}\left(1-v^{2}\right)
$$

In the case that the soil layer is of a finite depth, $h$, and that underlying the compressible layer is a non-compressible rigid foundation, there will be a reduction in the elastic settlement of the layer equal to the amount of settlement that would occur below depth $h$ in the case that the layer was semi-infinite. In other words, 


$$
\begin{gathered}
S_{e}=\int_{0}^{\infty} \varepsilon_{z} d z-\int_{h}^{\infty} \varepsilon_{z} d z \\
\text { or } \\
S_{e}=S_{e(z=0)}-S_{e(z=h)}
\end{gathered}
$$

Egorov further described this relationship by making the assumption that no friction existed between the elastic layer and the rigid layer to further characterize the elastic settlement of a finite layer. He gave relationships for the settlement below the center of circular loadings on the surface of a compressible layer of finite depth (Das, 1999). For the settlement below the center of a flexible plate and that below a rigid plate, respectively, he gives:

$$
\begin{gathered}
S_{e}(\text { center }, \text { flexible })=\frac{R q}{E_{s}}\left(1-v^{2}\right) \alpha_{1} \\
S_{e}(\text { center }, \text { rigid })=\frac{R q}{E_{s}}\left(1-v^{2}\right) \alpha_{2}
\end{gathered}
$$

In these equations, the values of $\alpha_{1}$ and $\alpha_{2}$ are defined based upon the ratio of the depth of the layer to the radius of the loading plate, as shown in Table 2.2.

\subsubsection{AASHTO and PCA Methods}

The AASHTO and PCA design philosophies are very similar in their approaches to thickness design for PCC pavements on soil bases and subbases. Both use an overall stiffness, or composite $k$-value, in units of $p s i$, to represent the overall stiffness of the support. In general, this can be obtained experimentally via plate bearing tests, as described in Section 2.6, through correlation with another property, such as the California Bearing Ratio (CBR), or by combining the effects of each of the multiple layers of the system.

The general AASHTO procedures for combining the support from the subgrade and subbase layers to determine an overall composite $k$-value of the system can be summarized as follows:

- STEP 1: Determine the Roadbed Soil Resilient Modulus, $\mathrm{M}_{\mathrm{R}}$, expressed in units of psi. This is to be done using the results of laboratory resilient modulus tests (AASHTO T 274).

- STEP 2: Determine the stiffness characteristics of the subbase material used. AASHTO specifies the elastic modulus value, designated $E_{S B}$, which has units of psi. 
For unbound base courses, $E_{S B}$ can be equivalent to the resilient modulus, which was discussed in Section 2.5; for bound materials either an indirect tensile test or compressive tests are often used.

- STEP 3: Using the material properties of Steps 1 and 2, along with the thickness of the subbase, the combined stiffness of the subbase and subgrade, $\mathrm{k}_{\infty}$, can be determined using Figure 2.13. This is done under a number of conditions to represent seasonal variations in behavior.

- STEP 4: Adjustments to each composite $k$-value are made for the presence of an incompressible layer if within a depth of $10 \mathrm{ft}$ below the top of the subgrade. At that point, a slab thickness is assumed, and all monthly composite $k$-values are combined based upon the average of relative damages which is expected each month for that pavement thickness (see the AASHTO guide for more details on this procedure).

The philosophy of the PCA method for representing the combined k-value is similar to that of Steps 1-3 of the AASHTO method described above, with some exceptions. First of all, for Step \#1 PCA uses a $k$-value to represent the subgrade stiffness, which can be determined through plate bearing tests or correlated to another property using relationships such as:

$$
\boldsymbol{k}=\mathbf{M}_{\mathbf{R}} / 19.4 \quad \text { (from AASHTO) }
$$

Secondly, the characterization of the subbase in Step \#2 is reduced to a general definition of this as either bound or unbound (with the exception of lean concrete subbases, in which case a more complex analysis is done). Third, to represent the approximate relationships between the subgrade stiffness, the subbase stiffness, the subbase depth, and the corresponding composite stiffness, Table 2.3 can be used.

Another difference between the techniques exists in that while AASHTO guidelines account for seasonal variations by specifying the determination of typical monthly values of the composite $k$-value to be combined at a later point, PCA simply specifies the use of typical summer or fall values to represent the average support behavior throughout the year.

It can be seen from Table 2.3 that the use of thin, untreated subbases results in a relatively low increase in the overall stiffness at the top of the subbase layer as compared to the original subgrade $k$-value; for this reason PCA does not recommend using untreated subbases for the sole purpose of increasing the pavement support, as it is not generally economical to do so (PCA, 1984). 


\subsection{Summary}

For the preliminary phase of this investigation, a lot of background information was obtained with regards to laboratory tests and analyses pertaining to vertical stiffness characterization of highway support systems. In particular, uniaxial compression tests can be conducted to characterize the general stress-strain relationships of Roadrain. A basic Finite Element Model was created to determine that the typical stresses to which a layer directly below a rigid pavement section would generally be around or below $70 \mathrm{psi}$, since the model did not include stress-reducing shear transfer devices present in most concrete pavements. Also, cyclic loading tests will determine the Resilient Modulus of the material. These characteristics are useful for the overall characterization of the roadbed support when implementing a Roadrain layer into a rigid pavement design; some of the techniques to do so have been discussed in this section.

Plate bearing tests are often used for in-situ assessment of pavement support. This is in compliance with both AASHTO and PCA rigid pavement design techniques. This test is used either to obtain or to verify values for the modulus of subgrade reaction, $k$, for the soil. The composite $k$-value, which takes into account the stiffness contributions of all supporting layers, is used for calculations pertaining to the necessary slab thickness. Methods for predicting the settlement for a support system, which can be used to predict the composite $k$-value of the soil system, were introduced in this chapter and will be discussed again later in the report. The numerical techniques for determining elastic settlement that were discussed were limited in the sense that these included methods for single layers of soil only, while most pavement systems include multiple layers. PCA and AASHTO give approximate relationships between subbase, subgrade, and overall stiffnesses for many applications, but these cannot account for the stiffness contributions of very thin layers, such as the addition of the $1 / 2$-inch-thick Roadrain layer. 
Table 2.1: Material Properties and Element Sizes Used for FEM Representation for Determining Typical Range of Non-Dowelled JPCP Subbase Stresses under Service Loads

\begin{tabular}{|c|c|c|c|c|c|}
\hline Component & $\begin{array}{c}\text { Outer } \\
\text { Dimensions }\end{array}$ & Material Type & $\begin{array}{c}\text { Young's } \\
\text { modulus, psi }\end{array}$ & $\begin{array}{l}\text { Poisson's } \\
\text { ratio }\end{array}$ & $\begin{array}{c}\text { Element } \\
\text { Dimensions }\end{array}$ \\
\hline Concrete Slab & $15^{\prime} \times 12^{\prime} \times 8^{\prime \prime}$ & Concrete & $3.5 \times 10^{6}$ & 0.15 & $6 " \times 6 " \times 2 "$ \\
\hline Subbase & $21^{\prime} \times 18^{\prime} \times 6 "$ & Granular Soil & $1.0 \times 10^{6}$ & 0.2 & $6 " \times 6 " \times 2 "$ \\
\hline Subgrade & $21^{\prime} \times 18^{\prime} \times 18^{\prime \prime}$ & Well-Graded Soil & $0.5 \times 10^{6}$ & 0.35 & $6 " \times 6 " \times 2 "$ \\
\hline
\end{tabular}

Table 2.2: Variations of Egorov's Constants for Calculating Settlement below the Center of a Circular Plate [Source: Das, 1999]

\begin{tabular}{|c|c|c|}
\hline $\boldsymbol{h} / \boldsymbol{R}$ & $\alpha_{1}$ & $\alpha_{2}$ \\
\hline 0 & 0 & 0 \\
\hline 0.5 & 0.52 & 0.45 \\
\hline 1.0 & 1.00 & 0.79 \\
\hline 2.0 & 1.44 & 1.16 \\
\hline 3.0 & 1.62 & 1.32 \\
\hline 5.0 & 1.78 & 1.48 \\
\hline 10.0 & 1.88 & 1.64 \\
\hline
\end{tabular}


Table 2.3: PCA Design [Composite] $k$-values When Considering the Effects of (a) Untreated Subbases, and (b) Cement-Treated Subbases of Varying Thicknesses [Source: PCA , 1984]

\begin{tabular}{|c|c|c|c|c|}
\hline $\begin{array}{c}\text { Subgrade } k \text {-value, } \\
\text { pci }\end{array}$ & \multicolumn{4}{|c|}{ [Composite] Subbase k-value, pci } \\
\hline 50 & $\mathbf{4}$ in. & $\mathbf{6}$ in. & $\mathbf{9}$ in. & $\mathbf{1 2}$ in. \\
\hline 100 & 65 & 75 & 85 & 110 \\
\hline 200 & 130 & 140 & 160 & 190 \\
\hline 300 & 220 & 230 & 270 & 320 \\
\hline
\end{tabular}

(a)

\begin{tabular}{|c|c|c|c|c|}
\hline $\begin{array}{c}\text { Subgrade } k \text {-value, } \\
\text { pci }\end{array}$ & \multicolumn{4}{|c|}{ [Composite] Subbase k-value, pci } \\
\hline 50 & $\mathbf{4}$ in. & $\mathbf{6}$ in. & $\mathbf{8}$ in. & 10 in. \\
\hline 100 & 170 & 230 & 310 & 390 \\
\hline 200 & 280 & 400 & 520 & 640 \\
\hline
\end{tabular}

(b) 


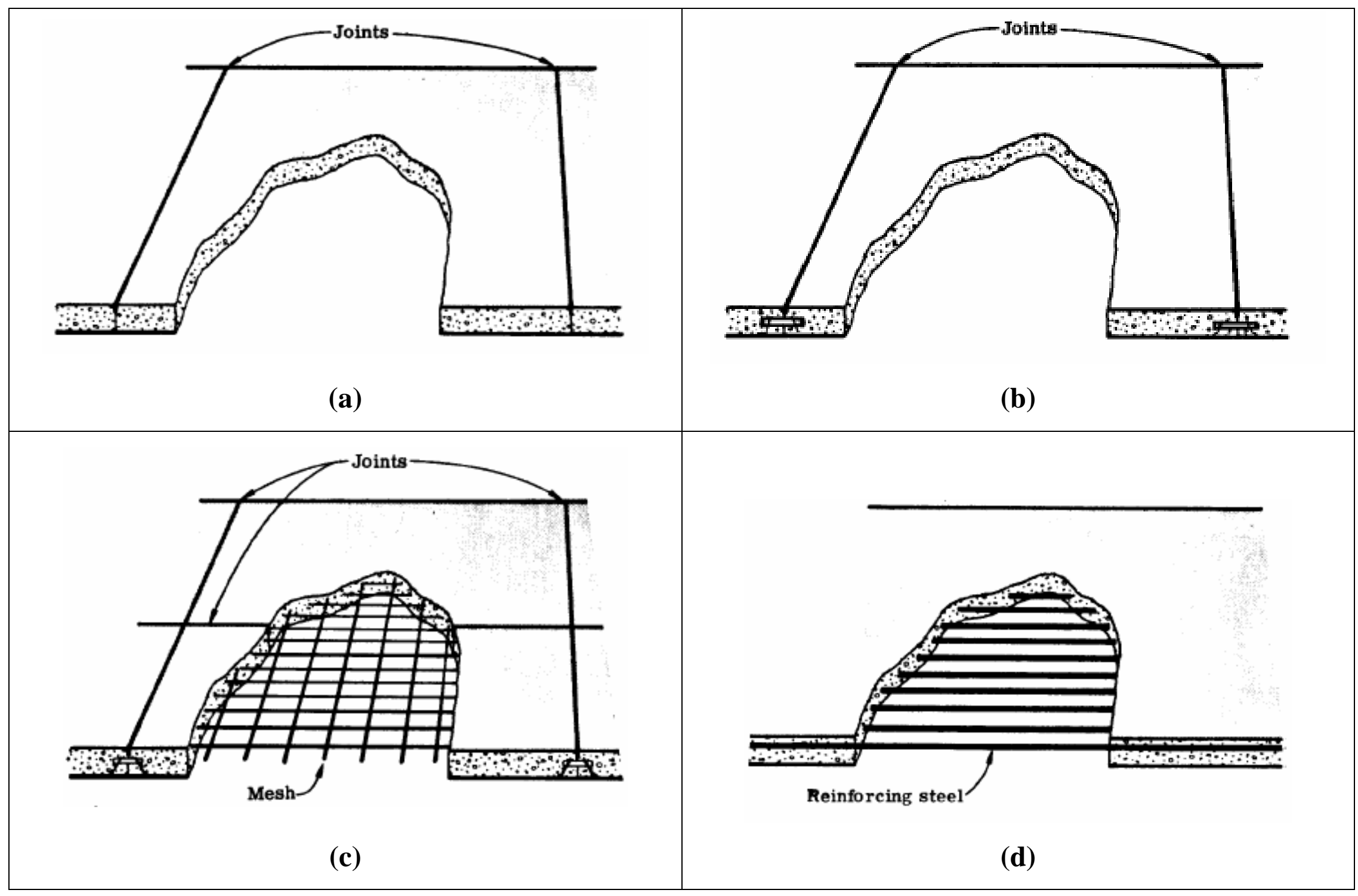

Figure 2.1: Various Types of PCC Pavements, as Defined by the Use of Saw-Cut Joints and Steel Reinforcement: (a) Jointed Plain

Concrete Pavement, or JPCP, (b) dowelled JPCP, (c) Jointed Reinforced Concrete Pavement, or JRCP, and (d) Continuously Reinforced Concrete Pavement, or CRCP. 


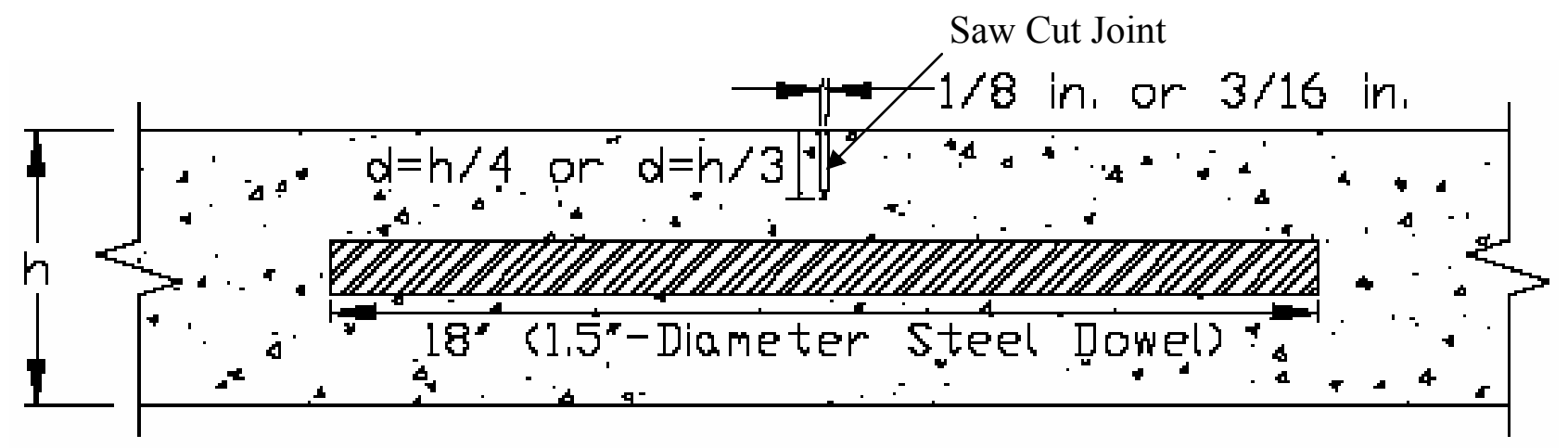

Figure 2.2: Typical Cross-Section of a Dowelled JPCP Saw-Cut Joint [SOURCE: Chen et al.]
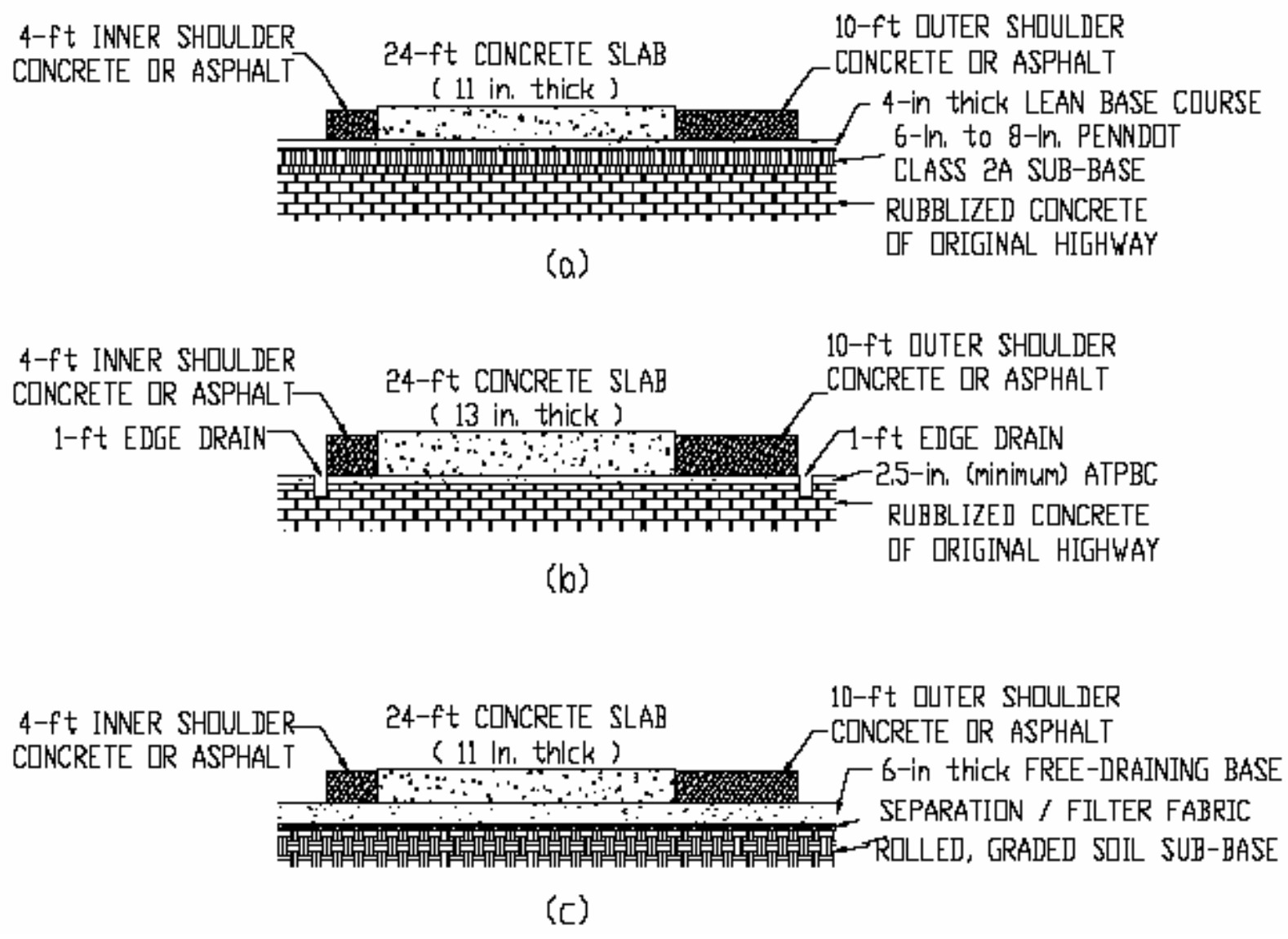

Figures 2.3: Some Pavement Support Layers Used for Various Highway Constructions in (a),

(b) South-western Pennsylvania, and (c) Central West Virginia

[Source: Chen et. al] 


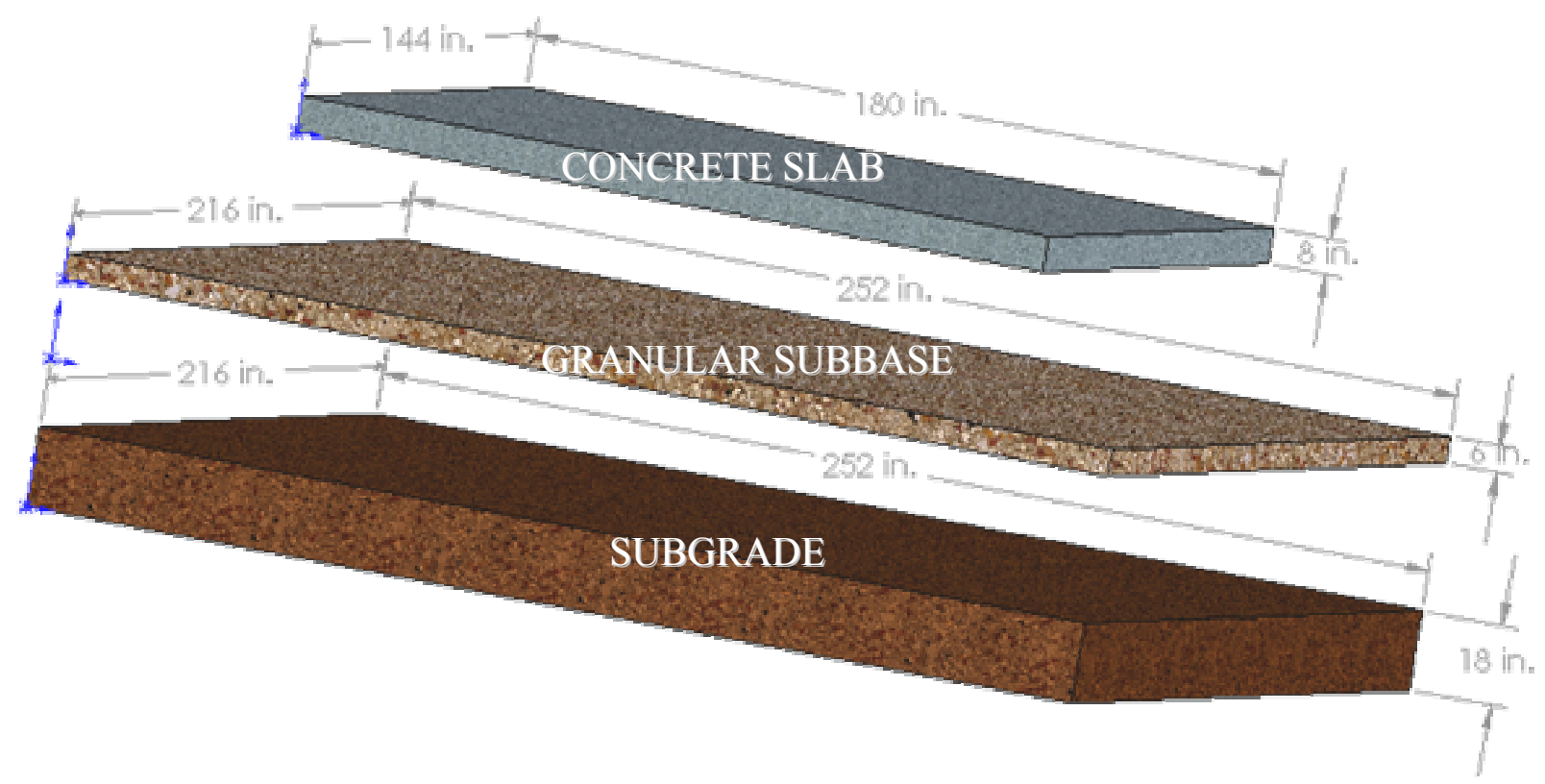

Figures 2.4: Exploded View of the Material Layers Used for the Finite Element Analysis Described in Section 2.3

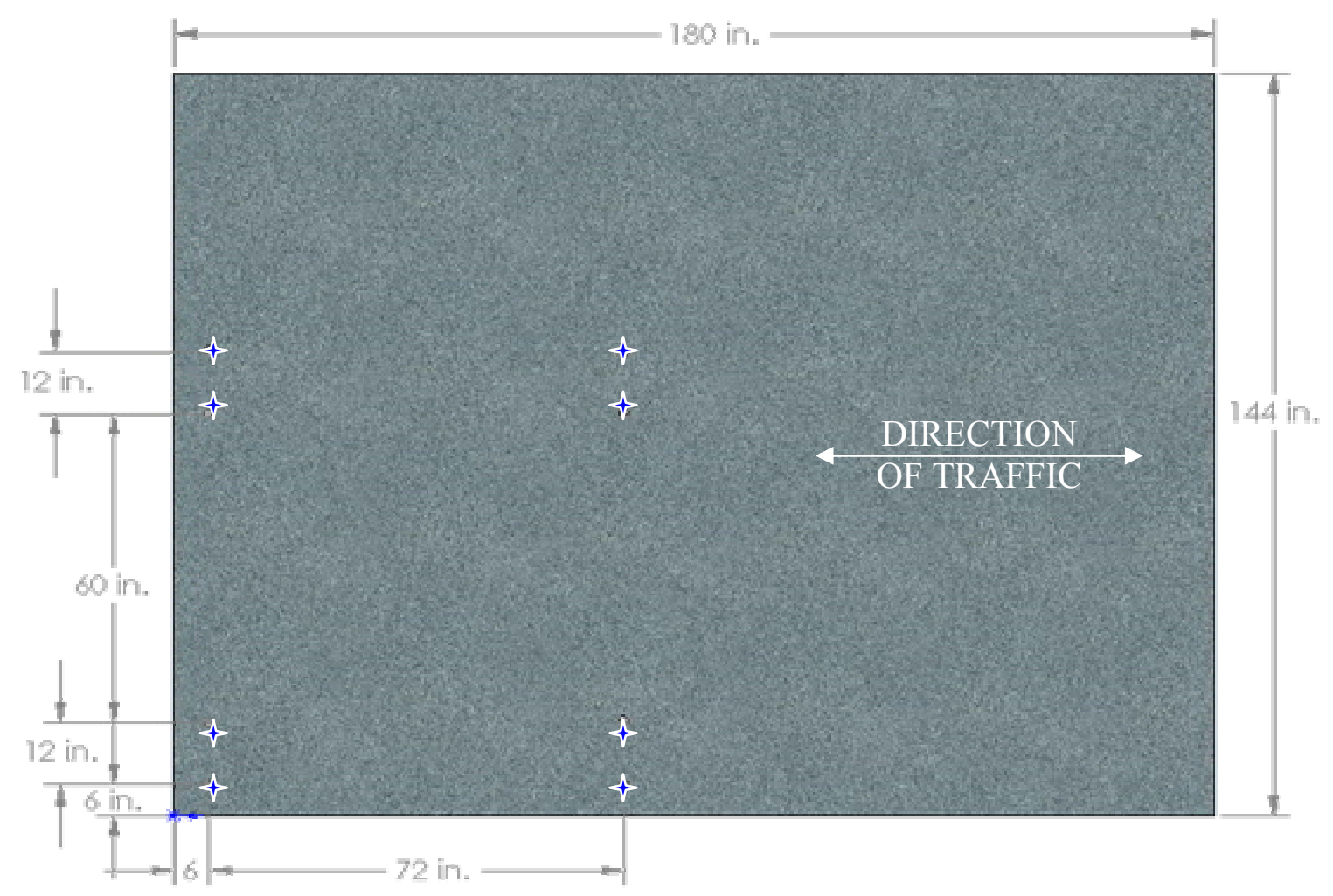

Figure 2.5: Schematic Diagram of the Locations of 5kip Point Loads Used for FE Modeling to Simulate a Dual-Tandem Truck Axle Configuration (Top View of Concrete Slab). 


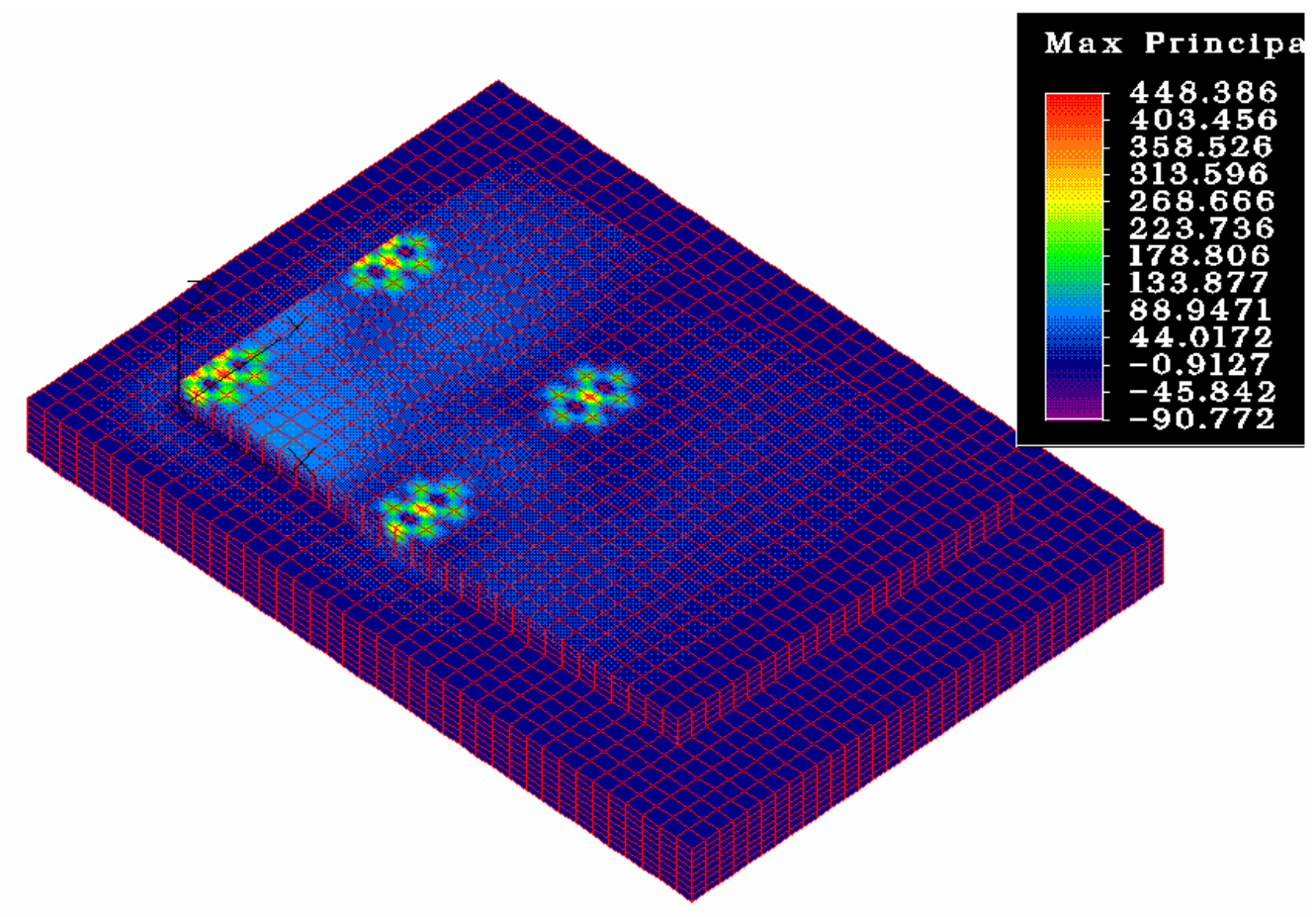

(a)

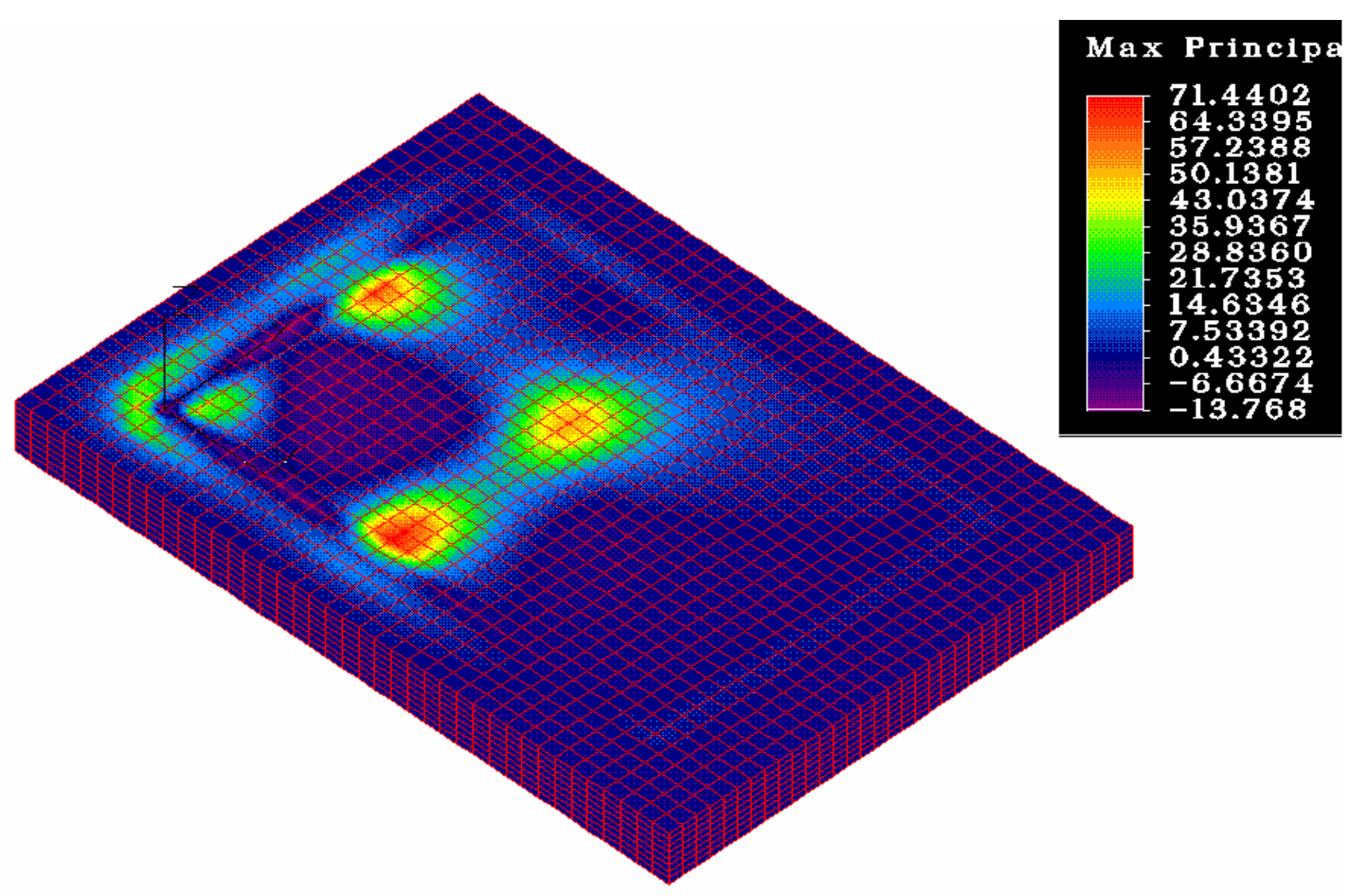

(b)

Figures 2.6: Maximum Principal Stresses obtained from the Finite Element Modeling Described in Section 2.3: (a) Shown with Slab, (b) Shown with Slab Removed to Expose Subbase Surface. 


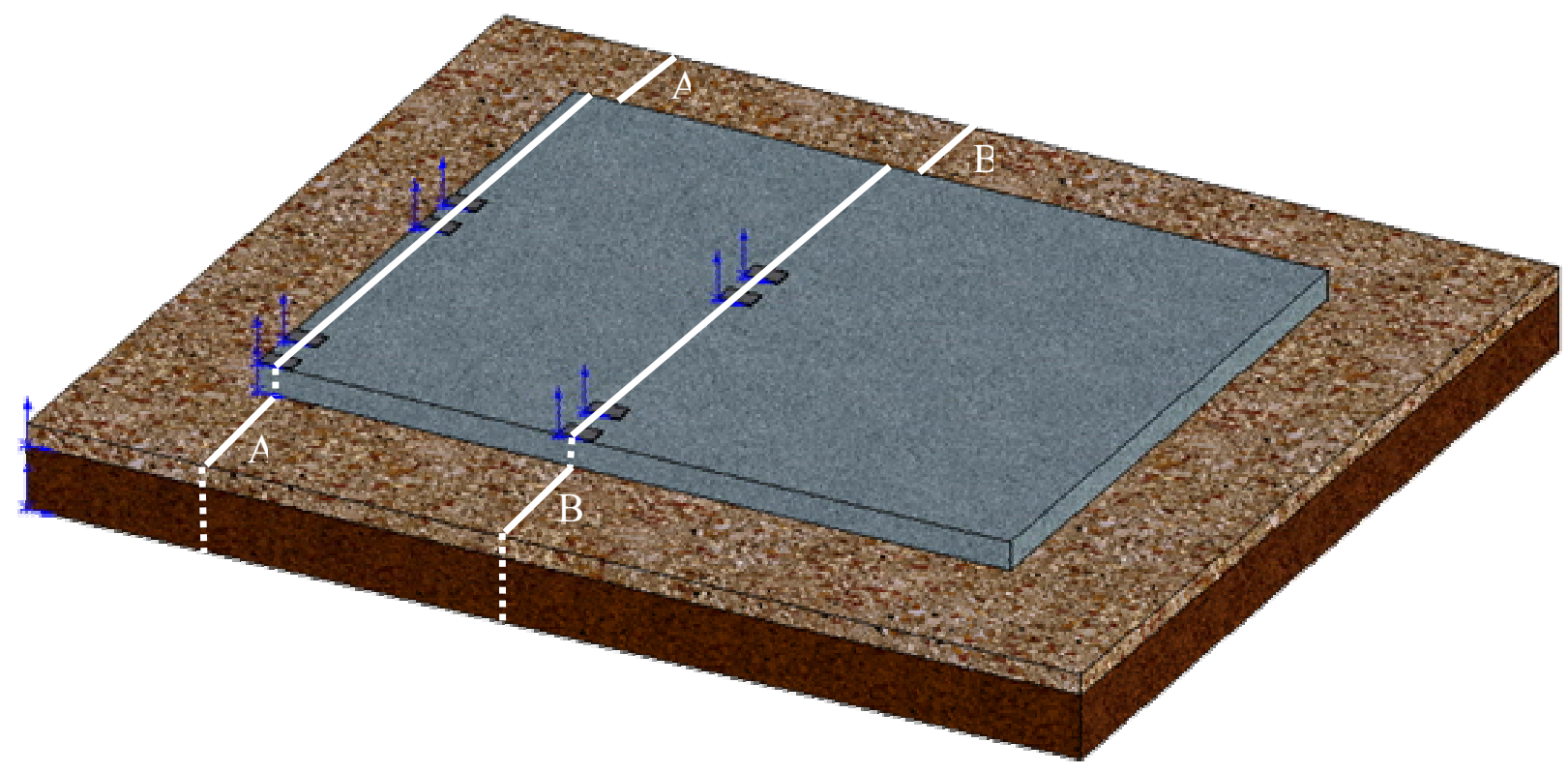

(a)

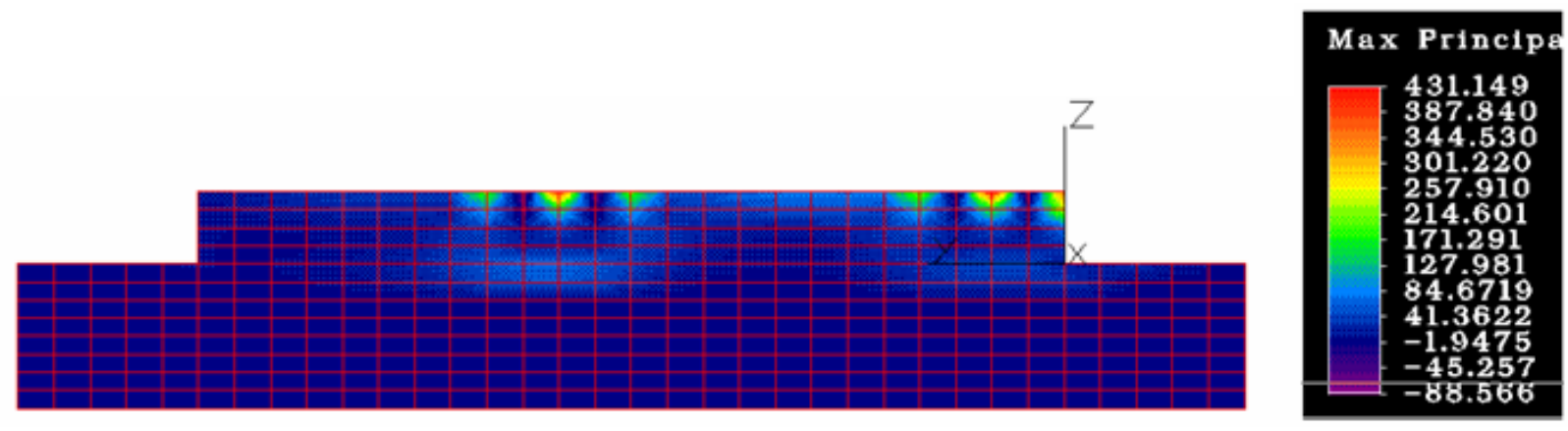

(b)
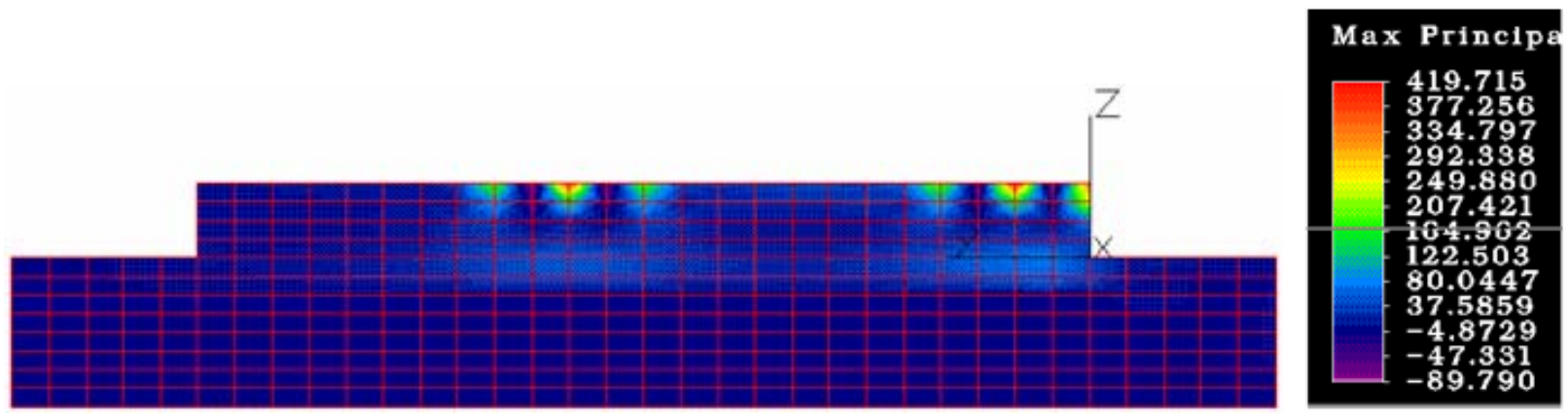

(c)

Figures 2.7: Maximum Principal Stresses at Selected Cross-Sections from FEM Results: (a) Locations of Cross-Sections, (b) Stresses at Section A-A, and (b) Stresses at Section B-B. 


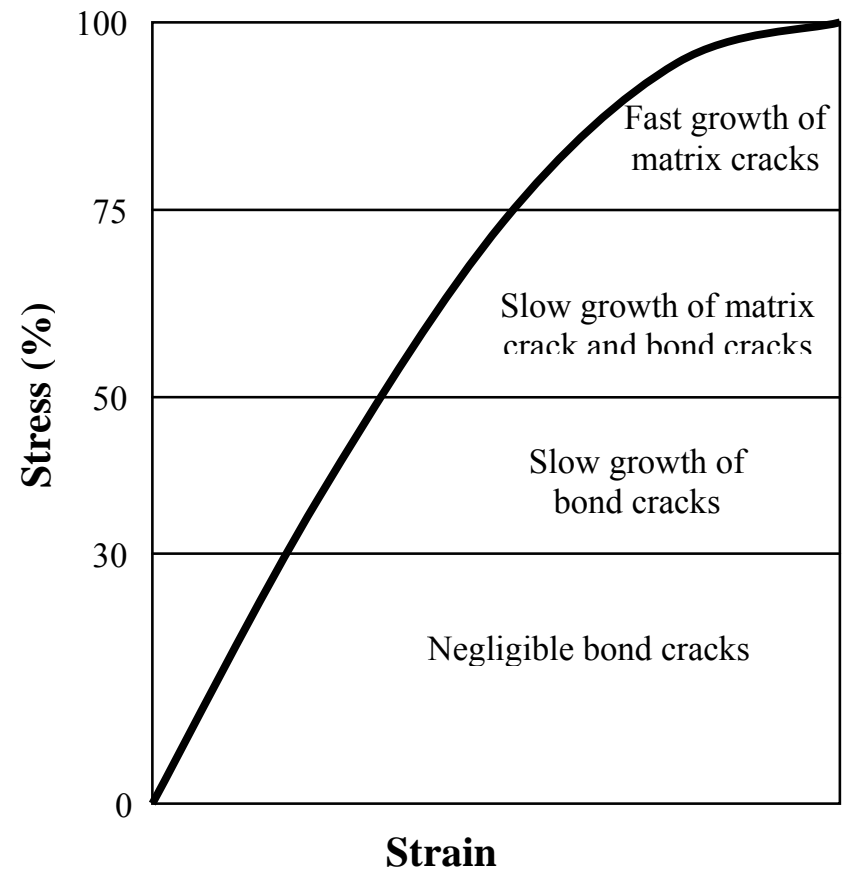

(a)



(b)

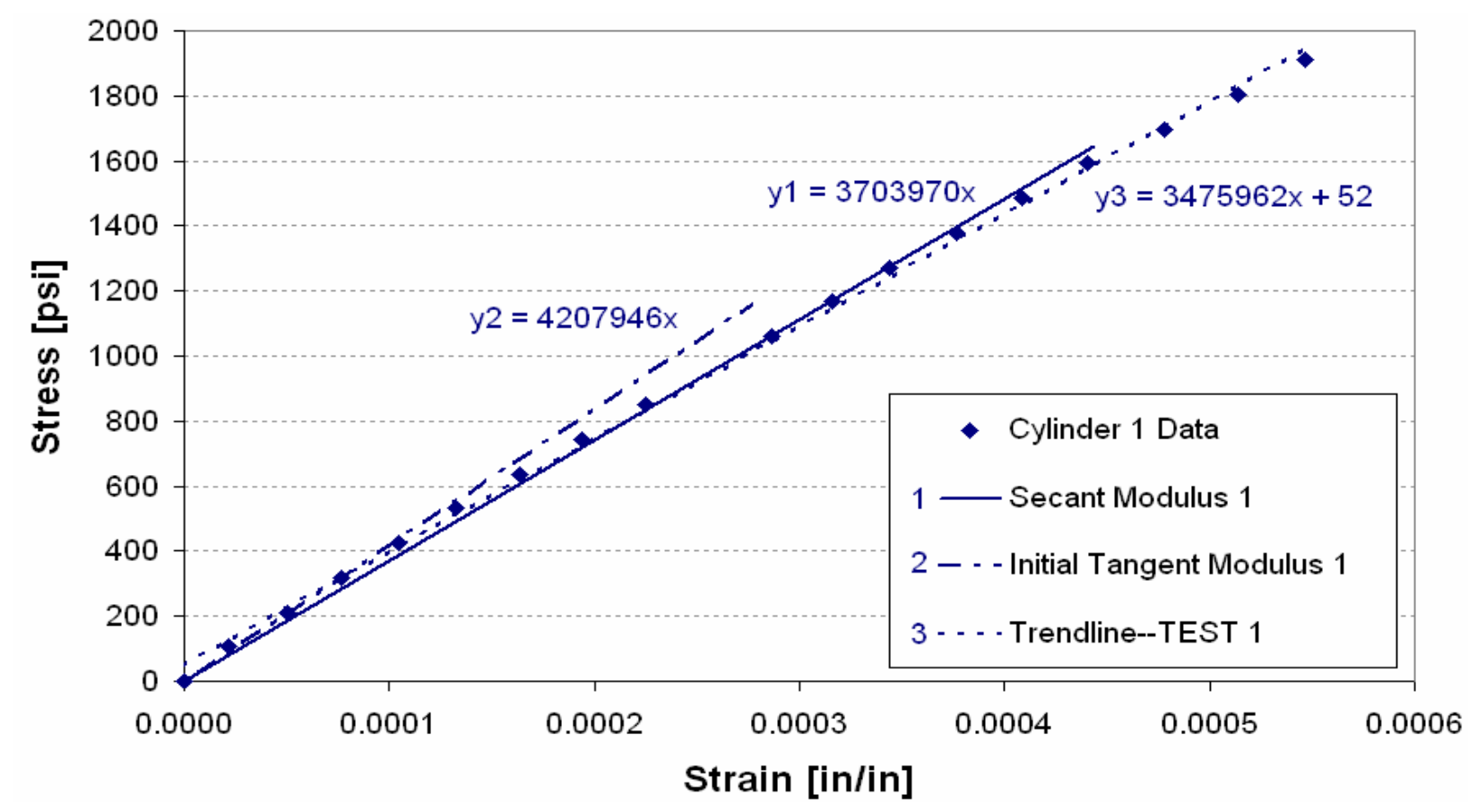

(c)

Figures 2.8: Examples of Non-linear Stress-Strain Relationships Exhibited by Concrete: (a) General Behavior, (b) Some Typical Linear Approximations, and (c) Laboratory Data and Stiffness Calculations from the 28-Day Compression Test of a 6in.x12in. Concrete Cylinder 


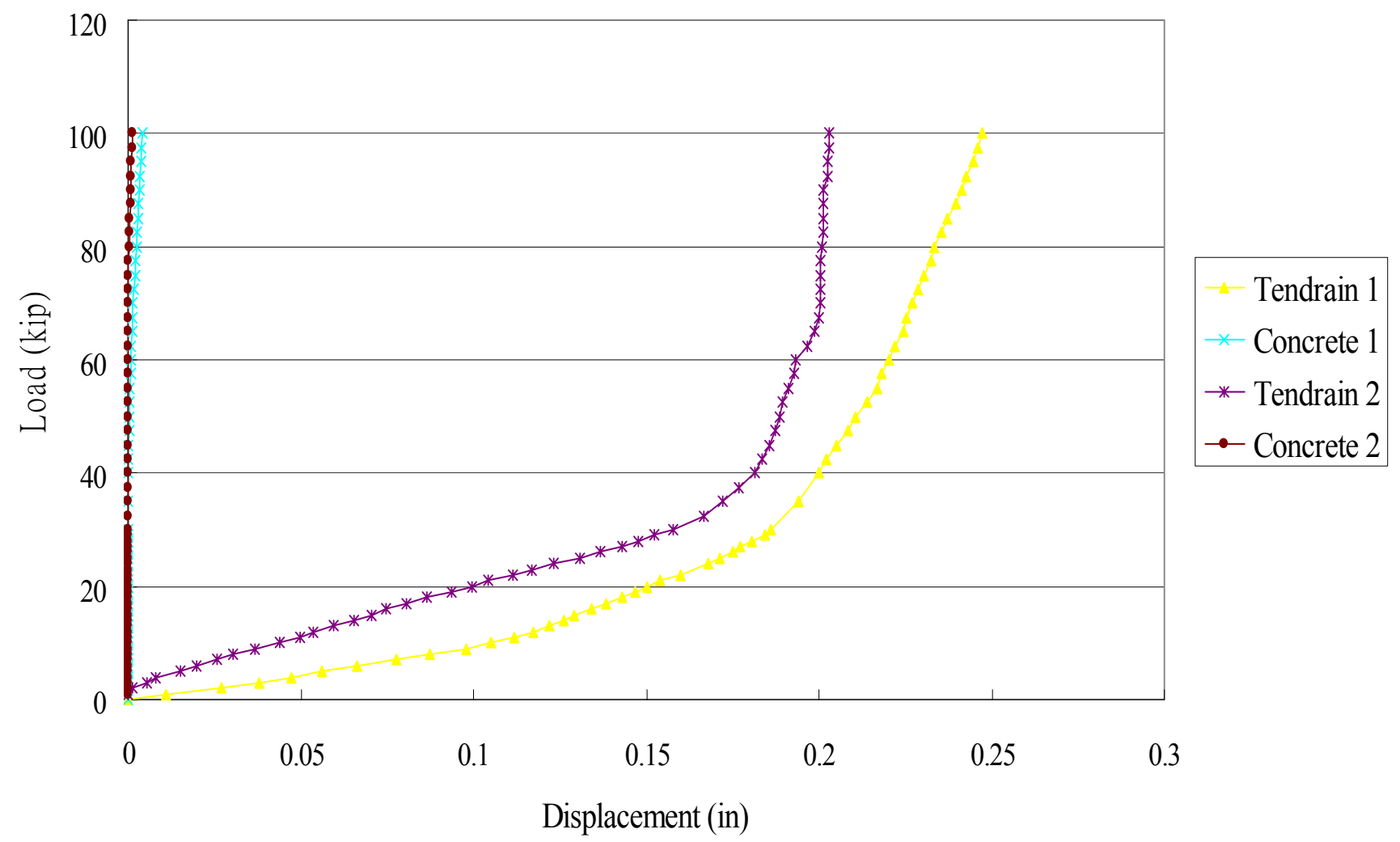

Figure 2.9: Results of Preliminary Compression Tests Done in Phase I using Two Roadrain [a.k.a. "Tendrain"] Samples Bonded to Concrete Cylinders [Source: Cook] 


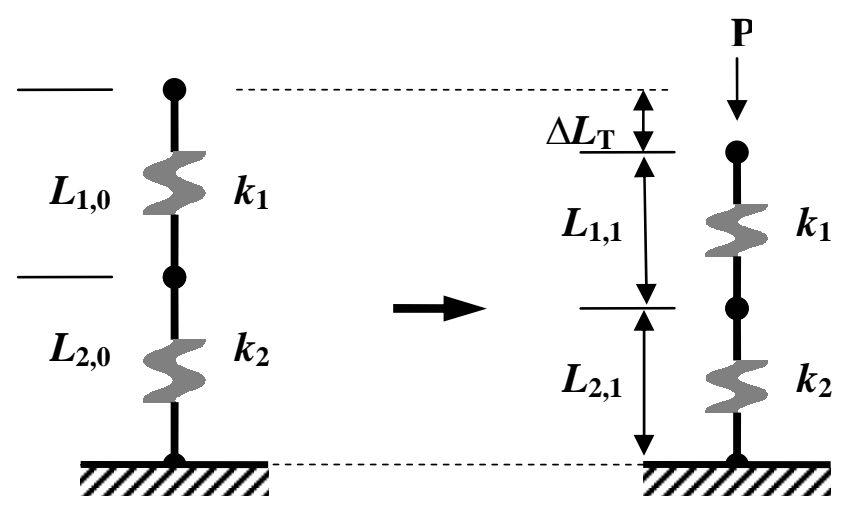

Non-Loaded Specimen

Loaded Specimen

$$
\Delta L_{\mathrm{T}}=\Delta L_{1}+\Delta L_{2}
$$

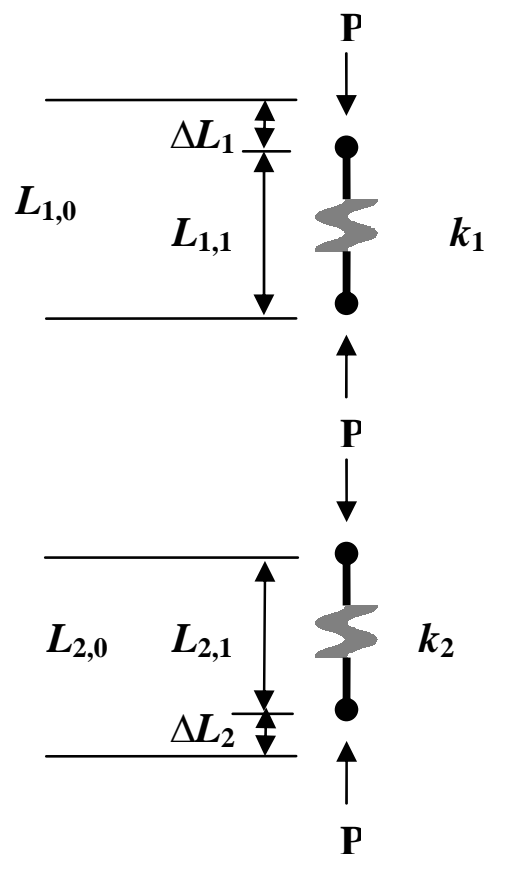

Idealized Forces from

Simplified Multi-Layer Model

$$
\begin{aligned}
& \Delta L_{1}=\mathrm{P} / k_{1} \\
& \Delta L_{2}=\mathrm{P} / k_{2}
\end{aligned}
$$

Figure 2.10: Simplified Model of a Uniaxial Compression Load Acting on a Two-Layered Specimen 




Figure 2.11: Initial Strain Behavior of a Material Which Undergoes Plastic Behavior under Repeated Loading [Based on Huang]

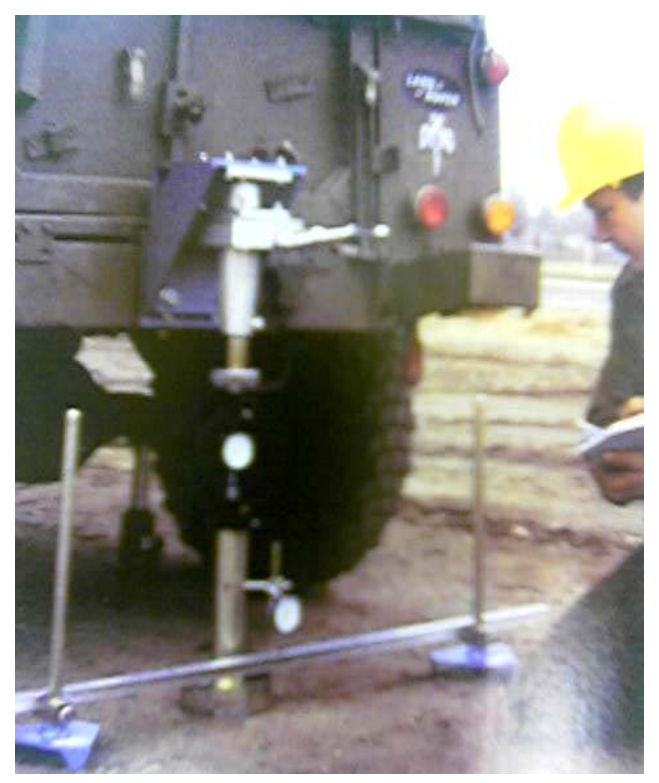

Figure 2.12: Picture of Field Plate Bearing Test Setup as Conducted in the Field [Source: ELE International] 


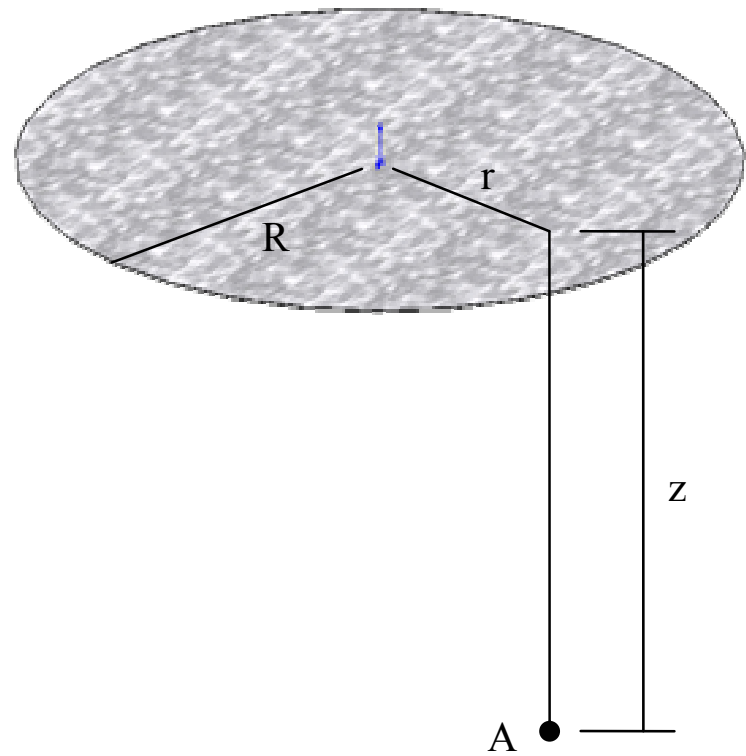

Figure 2.13: Basic Definitions of Typical Models Used for Determining the Stress Increase at Point "A" below a Uniformly Loaded Flexible Circular Area 
Example:

$$
\begin{aligned}
& D_{S B}=6 \text { inches } \\
& E_{S B}=20,000 \text { psi } \\
& M_{R}=7,000 \text { psi }
\end{aligned}
$$

Solution: $k_{\infty}=400 \mathrm{pci}$

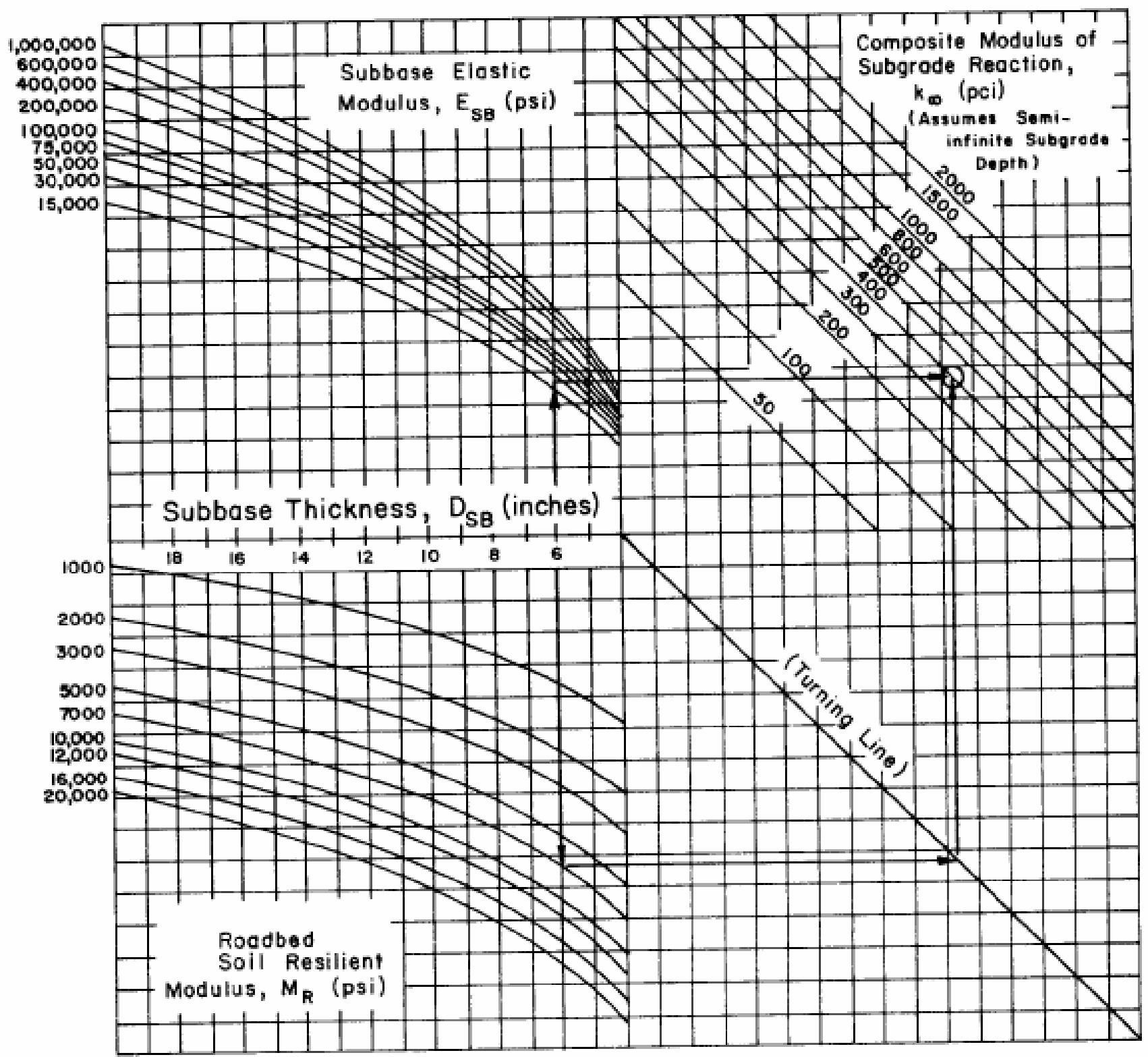

Figure 2.14: AASHTO Design Chart for Estimating Composite Modulus of Subgrade Reaction Assuming a Semi-Infinite Subgrade Depth [Source: AASHTO] 


\section{CHAPTER 3 \\ VERTICAL COMPRESSION TESTS}

\subsection{Overview}

To first characterize the behavior of Roadrain when it is subjected to vertical loading, various loading schemes were developed and implemented for the vertical compressive tests. The loadings were intended to allow for the determination of the nonlinear stress-strain relationship exhibited by Roadrain by simulating a variety of loading conditions which would account for various types of in-situ loadings. Primarily of interest were (1) the dependency of this non-linear relationship on the rate of loading, or creep effects, and (2) the increase in stiffness of the Roadrain after being exposed to multiple loads.

\subsection{Test Specimens}

A total of twelve Roadrain samples were tested for this portion of the study. These were divided into two batches, which will be referred to throughout this and subsequent chapters as Batch \#1 and Batch \#2.

The compressive specimens of both batches consisted of 12-inch tall x 6-inch diameter concrete cylinders cast upon samples of Roadrain. The Roadrain samples in all cases had approximate dimensions of 8 in. $x 8$ in. and were cut from a roll of roadway drainage geocomposite utilizing continuous fiber geotextiles. A schematic diagram of a specimen and a sample picture can be seen in Figures 3.1(a) and 3.1(b), respectively.

Two different mix designs were used for the concrete: one using a water-tocement ratio $(w / c)$ of 0.4 , and one using a $w / c$ of 0.57 . Each batch contained three cylinders with $w / c=0.4$ and three cylinders with $w / c=0.57$. Two additional cylinders, one for each $w / c$, were cast using molds with the bottoms still in tact; this was done to obtain the approximate ultimate compressive strength values, $f_{c}$, for each type of concrete.

\subsubsection{Concrete Mix Designs}

The concrete mix designs used in this investigation were based on West Virginia Department of Transportation (WVDOT) Class B mix specifications. This was done in 
attempts of getting concrete properties similar to that which would be achieved in the field. The two different water-to-cement ratios, $w / c, 0.4$ and 0.57 , corresponding to two different strengths of concrete, were used to investigate whether or not there would be a noticeable difference in the stiffness of the Roadrain with a softer material on one side. Neither mix used admixtures of any sort. The mix proportions for both mixes can be seen in Table 3.1 .

\subsubsection{Specimen Preparation}

The forms for the samples were created by first removing the bottom of a standard 6in. $x 12$ in. concrete mold, leaving essentially a 12 in.-long plastic tube which is open on both ends. This mold was then secured on one end to the Roadrain sample using clear silicone caulk. In preparation for casting of the concrete, the form was cleaned after the caulk had hardened, and a thin coat of oil was placed on the inside of the molds only, taking care to avoid application of oil to the Roadrain. The final step before casting was to carefully dampen the Roadrain layer itself, avoiding removal of the oil from the concrete forms.

The concrete was poured into the molds in three layers, with each layer being consolidated as specified by ASTM C 31; for Batch \#1, a vibrating rod was used for consolidation, and Batch \#2 was consolidated using 25 strokes of a tamping rod. The cylinder surface was then prepared by first striking off any extra concrete using a screed, and then finished with a trowel at the appropriate time. Finally, curing compound was applied to the surface after finishing, and the cylinders were then placed in a curing room with a constant temperature and humidity for curing. The samples were left to cure for in the curing room for a period of at least 28 days, until the compression tests were ready to be run. At that point, the plastic forms were cut and removed, leaving only the concrete and the Roadrain to which it had bonded.

Those cylinders which did not incorporate Roadrain were cast in the standard 6in.x12in. cylindrical molds with the bottoms still in tact, following preparation procedures similar to those described above. The forms were cleaned and oiled, and casting was done in layers, with the same consolidation and finishing techniques as the 
Batch \#2 specimens with Roadrain. The forms for these cylinders were also removed at the same time as those of the Batch \#2 specimens.

\subsection{Test Setup and Procedures}

\subsubsection{Equipment and Instrumentation}

All compression cylinders were tested using a Baldwin Compression/Tension Loading Machine to apply the required loads. This machine includes a self-contained load cell which was utilized to record the applied load throughout all Batch \#1 tests. In lieu of the Baldwin Machine load cell, an independent load cell was used in the Batch \#2 tests to measure loads, since it had a higher resolution at smaller loads than the Baldwin load cell.

In addition to the applied loads of all tests, various deflections were also measured. In the Batch \#1 tests, a concrete cylinder compressometer with a gage length of 6 in., utilizing a single vertical dial gage (a set-up in compliance with ASTM C 469), was used to measure the deflections necessary to make Modulus of Elasticity calculations for the concrete. Also, a Linear Variable Displacement Transducer, or LVDT, was used to measure the deflection of a section of the specimen equal to the thickness of the Roadrain plus approximately 2 in. of the concrete cylinder. A schematic diagram and a picture of the specimen with the appropriate measurement devices can be seen in Figures 3.2(a) and 3.3(b), respectively.

For the Batch \#2 tests, the Modulus of Elasticity of the concrete was not of

concern, so no dial gages were used on the concrete cylinder compressometer. (The reason for this is that the Batch \#1 results unveiled that the concrete had a much higher stiffness than the Roadrain, so a minor change in the concrete stiffness would not significantly alter the calculated Roadrain stiffness; this has been discussed in more detail in Chapter 2). Deflection measurements were taken for the Batch \#2 tests using two LVDTs. This was done in a similar fashion as that of the LVDT of the Batch \#1 tests, recording displacements of the Roadrain plus approximately 2 in. of concrete. A schematic diagram and a picture of this setup can be seen in Figures 3.3(a) and 3.3(b), respectively. 
It can be seen by comparing Figures 3.2 and 3.3 that the orientation of the test specimen was reversed, as they were flipped vertically after the Batch \#1 tests. This was done to get more precise displacement readings at small stress levels than could be achieved with the original orientation, since smaller loads were used for the Batch \#2 tests.

All digital data for the vertical compression tests was recorded using a Smartstrain 5000 data acquisition system. This data acquisition system was connected directly to a PC for ease of setting adjustments and data storage. The data collected with this system included that from the Baldwin machine load cell, all LVDTs, and the load cell used in the Batch \#2 tests. A picture of the setup as it appeared in the laboratory during the testing of the Batch \#1 specimens can be seen in Figure 3.4.

\subsubsection{Loading Schemes Overview}

The loading schemes devised for the compressive tests will be divided into three types: (1) Load-Release tests, (2) Repetitive Load-Release tests, and (3) Sustained loading tests. Each of these is intended to simulate a different type of loading to which the Roadrain will be exposed while in the field. The Load-Release tests give Roadrain's stress-strain relationship for the first time it is ever loaded only, such as during the initial construction phase of the highway; all Batch \#1 cylinders were all tested in this manner. The Repetitive Load-Release tests give the stress-strain relationship of Roadrain after it has already been exposed to a number of loads, as it would experience throughout its service life; four Batch \#2 cylinders, two of each $w / c$, were tested in this manner. To further eliminate the effects of creep on Roadrain stiffness, the release portions, or the unloading curves for all specimens from the Repetitive Load-Release tests were investigated as well. Finally, the Sustained loading tests simulate the stress-strain relationship of Roadrain which account for creep effects under a continuous load, which may occur in the case of slow moving or stopped traffic while the highway is in service; two Batch \#2 cylinders, one of each $w / c$, were tested in this manner.

Table 3.2 shows which method of loading each cylinder was exposed to during testing. The alpha-numeric designations given for the cylinders have the following form: 


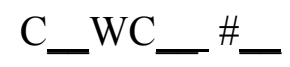

This terminology will be repeated throughout the remainder of this chapter. Using this convention, the first letter [C] and first number [1 or 2] signify the type of test and the batch number; the remainder of the letters and numbers represent the mix type [numbers 04 or 057 ] and the pre-designated specimen number [\#1, \#2, or \#3] (e.g.: C1WC057 \#1 would signify Compressive tests, Batch \# $\underline{1}$, concrete with $\underline{w} \underline{\underline{c}} \underline{0}=\underline{0} . \underline{57}$, cylinder designation $\# 1$, or in other words the first specimen from Batch \#1 with $w / c=0.57$ ).

\subsubsection{Procedure for Load-Release Tests}

The Load-Release test procedure is the simplest of the three procedures. Once the specimen to be tested was in place with all appropriate instrumentation secured, as described in Section 3.3.1 for Batch \#1, a compressive load was applied until the predetermined value was reached. At that point, the load was released completely. To obtain the target values for each mix, this procedure was done for the first cylinder of each mix to a target load that was conservatively estimated to be well below the maximum compressive strength of the concrete, $f_{c}$ '. The instrumentation was then removed and the cylinder was again loaded until failure in order to get a general idea of $f_{c}$, of each concrete mix. Then, using that information, a target load value for the remaining specimens of the respective concrete mix was established. The target loads and compressive strengths for each cylinder can be seen in Table 3.3.

Electronic data collection was done for each specimen from the time of initial loading until at least the point at which the load was completely released and continued in most cases until the majority of the elastic deformation had been recovered. Also, dial gage readings were taken at regular intervals during loading for determination of the Modulus of Elasticity of the concrete.

\subsubsection{Procedure for Repetitive Load-Release Tests}

For the Repetitive Load-Release tests, the specimen to be tested was placed and instrumented as described in Section 3.3.1 for Batch \#2 specimens. At that point, loads were applied, held at a constant level until material deformation slowed considerably, and 
released completely. After the rebound deflection of the Roadrain had stabilized, this process was repeated for another load. The loading patterns were unique for each specimen tested in this manner; these can be seen in Table 3.4.

Electronic data collection was done for each specimen continuously from the time of initial loading until the point at which rebound deflections had stabilized for the last load applied. No dial gage readings were taken for the Repetitive Load-Release Tests, since theory and previous results showed that a small variation in the concrete stiffness would produce negligible changes in the calculated stiffness of the Roadrain; this is due to the fact that the strength of concrete greatly exceeds that of the Roadrain, especially at small loads, as was seen in the original Load-Release tests. However, the two concrete cylinders that were cast without Roadrain were still tested until failure in order to verify the approximate values of $f_{c}$ ' and to check this assumption.

Smaller load levels were used for the Repetitive Load-Release tests than were used for the original Load-Release tests since the stress-strain relationship of Roadrain when subjected to stress levels which it would incur in the field was of primary importance; the maximum stress level applied for the Repetitive Load-Release tests was around 140 psi as opposed to the maximum around 4,000 psi from the original LoadRelease tests. Pavement layer stress levels were briefly discussed in Chapter 2.

\subsubsection{Procedure for Sustained Loading Tests}

The setup and instrumentation for the Sustained loading tests was identical to those of the Repetitive Load-Release tests. For the test, a small load was first applied to the specimen; this was held constant until the rate of change of the deflection approached a very small value. At that point, without releasing the load, the load value was increased to a higher value, and then held constant until deformation virtually ceased. This process was continued for various increasing load values (see Table 3.5) until the maximum load to be applied was reached. At that point, the load was released completely. After a point at which the rebound deflection became very steady, this entire process was repeated using the same load pattern. This loading was done three times for both specimens that were tested in this manner. 
Electronic data collection was done for each specimen continuously from the time of initial loading until the point at which rebound deflections had stabilized after the release of the last load applied. No dial gage readings were taken for the Repetitive Load-Release Tests for the reasons described in the previous section.

\subsection{Experimental Results}

\subsubsection{Overview}

Load data and deflection data were collected from all vertical compression tests using the instrumentation and procedures described in Section 3.3. These loads and deflections were then converted to stresses and strains using the knowledge that the area of loading, or area of the concrete cylinder, is $28.27 \mathrm{in}^{2}$, and that the thickness of uncompressed Roadrain is 0.5 in. The data sets collected from all test specimens that were subjected to the same type of loading were then used to establish the non-linear Roadrain stress-strain relationships for the possible in-situ loading conditions that were discussed in Section 3.3.2.

\subsubsection{Load-Release Test Results}

The stress-strain data of the concrete as obtained from the dial gage and Baldwin machine readings throughout the Load-Release Tests was very scattered and therefore believed to be inaccurate. Suspicion of this inaccuracy was based on the observation in the laboratory that at some points during testing the needle on the compressometer dial gage would exhibit a large variations in the rate at which it moved, even sometimes completely stopping, as the loading continued to increase at a uniform rate. Therefore the data collected using this gage was scrapped and another method of approximating the concrete's modulus of elasticity was employed, namely the ACI equation:

$$
\mathrm{E}_{\mathrm{c}}=57,000^{*}\left(\mathrm{f}_{\mathrm{c}}\right)^{0.5} \quad[p s i]
$$

Using this equation, along with the ultimate compressive stresses applied to the one cylinder for each $w / c$ which was broken (see Table 3.3), it was determined that the mixes with $w / c=0.4$ and $w / c=0.57$ had modulus values of approximately $4.6 \times 10^{6} \mathrm{psi}$ and $3.83 \times 10^{6} \mathrm{psi}$, respectively. 
Figures 3.5(a) and 3.5(b) show the stress-strain curves obtained from the groups of specimen using $w / c=0.4$ and $w / c=0.57$, respectively. It can be seen from these curves that while there was some deviation between curves of different cylinders, especially at very high stress levels, this deviation was not primarily a result of the differences in the concrete strength. In fact, the specimens using the higher strength concrete $(w / c=0.4)$, which one would expect to deform less than those with weaker concrete, actually on the whole experienced higher strains at the same stress levels than those using weaker concrete. This leads to the conclusion that the overall variations in specimen behavior can be mainly attributed to the behavior of the Roadrain itself. This point is reinforced when comparing the curves of Specimens C1WC04 \#1 and C1WC057 \#1; while it is known that the concrete of Specimen C1WC04 \#1 has a higher measured compressive strength than that of Specimen C1WC057\#1, it can be seen that the overall deflection of Specimen C1WC04 \#1 is higher at a load level of 1,500 psi.

Regardless of the variations at very high loads, the performances of most specimens at lower loads were fairly consistent. It can be seen from Figure 3.6, a plot of the stress-strain data from all six test specimens, that five out of the six specimens have very similar displacements up to $1,000 \mathrm{psi}$, and their paths only start to deviate shortly thereafter. Furthermore, four of the six have reasonably similar relationships up to 2,000 psi. At best-fit polynomial curve of the fourth power was created for the sets of data for loads up to a stress level of 2,000 psi. This curve represents the typical behavior of Roadrain (which has not previously been loaded) when subjected to a brief, static load up to 2,000 psi. This gives the following stress-strain relationship:

$$
\sigma_{\mathrm{y}}=5,114 \cdot \varepsilon_{\mathrm{y}}{ }^{2}+234 \cdot \varepsilon_{\mathrm{y}}
$$

which is applicable for the initial short-term, static loading of Roadrain (up to 2,000 psi).

\subsubsection{Repetitive Load-Release Test Results: Loading Curves}

The individual stress-strain curves for the Repetitive Load-Release tests had a similar shape as those of the original Load-Release tests. However, since the deflections were monitored continuously from the beginning of each test, plastic deformations were present at the beginning of each loading cycle. Figure 3.8 shows an example of the results for one of the specimens; the raw data for the rest of these tests is in Appendix A. 
In order to better understand the effects from multiple loadings on the Roadrain stiffness, the plastic deformation was eliminated such that all loadings started with a deformation equal to zero. Figures 3.9(a) through 3.9(e) show the loading portions of the stress-strain curves after the adjustments removing the plastic deformations. From these figures it can clearly be seen that the Roadrain is subjected to higher deformations the first time that it is loaded to a maximum value, but it seems that the stress-strain curve normalizes after only a few loads exceeding that value.

To illustrate the point, it is evident looking at Figure 3.9(d) that once the loading (i.e. the first 1,500-lb and 2,500-lb applications) exceeds the maximum value to which it had been loaded previously, there is significantly more deflection than the second application of the same load. Furthermore, once the maximum loading has reached or exceeded that which is being applied (i.e. the second 1,500-lb and 2,500-lb applications), the stress-strain relationship is fairly consistent and will increase only slightly; it can be noted that even the 4,000-1b application has approximately the same stress-strain progression as the latter 1,500-lb and 2,500-lb applications, at least until the point at which loading exceeded the previous maximum of 2,500 lbs. The same trends are evident in the other Repetitive Load-Release tests, as well.

Observing these patterns, it was apparent that the eventual stress-strain behavior of Roadrain in the field could be described by a characteristic curve comprised of loads to which it has already been subjected multiple times. For this reason, the stress-strain curves corresponding to the final application of each load for each test specimen were plotted on the same graph as a means of characterizing this behavior (see Figure 3.10a). From this plot, a polynomial best-fit curve was created using the data points from all tests in order to represent the stress-strain relationship shown in Figure 3.10(b); this gives the following equation to describe the stress-strain relationship:

$$
\sigma_{\mathrm{y}}=12,156 \cdot \varepsilon_{\mathrm{y}}{ }^{2}
$$

which is eventually applicable for repeated application of short-term, static loads up to a stress level of $100 \mathrm{psi}$. 


\subsubsection{Repetitive Load-Release Test Results: Unloading Curves}

In addition to the loading curves of the Repetitive Load-Release test data, the release portions, or the unloading curves, were also investigated. This was done in attempts of further lessening the time-dependent effects of creep on the Roadrain stiffness obtained. To do this, the strain values of the unloading curves were taken as the rebound deflections, with displacements equal to the maximum deflection for that loading minus the current value of displacement, such that all rebound deflections possess a positive value. A sample of these plots can be seen in Figure 3.11.

From these plots, it can be seen that the unloading curves, unlike the loading curves, are very repeatable for every load level and are not dependent upon the number of loads to which the Roadrain has been subjected, as these curves are the same for the first and last load applications. There is also an increased consistency over the loading curves with respect to the results from each specimen corresponding to a given load. Figures 3.12(a) through 3.12(g) show plots of the combined rebound stress-strain data from all applicable cylinders corresponding to unloading from seven given stress levels.

Once again, polynomial best-fit curves were applied to these figures to describe the stress-strain behavior, this time further reducing the effects of creep on the results. A comparison of these characteristic equations can be seen in Figure 3.13. Although these equations adequately describe the stress-strain relationships in the stress region, it can be seen that some of these do a less-than-adequate job in predicting the strain values at stresses higher than those tested: in particular those curves corresponding to unloading from $250 \mathrm{lbs}$ and from $500 \mathrm{lbs}$. While the other five equations are for the most part parallel at any given load, there is most likely insufficient data in these small stress ranges to accurately predict far beyond the given range. The comparison of the Tangent Moduli obtained using these equations seen in Figure 3.14 reinforces this point, as the slopes of the equations corresponding to the upper five unloading stresses are comparable throughout a range up to $140 \mathrm{psi}$, whereas those from the lower two ranges could be considered outliers even at stress levels below $60 \mathrm{psi}$. 


\subsubsection{Sustained Loading Results}

The Sustained loading tests were done to account for creep effects that may occur due to such things as slow moving or stopped traffic on the highway. For this reason, load levels throughout the test were held for an adequate amount of time to allow most of the creep effects to take place. The raw data from the three tests of each specimen can be seen in Figures 3.15(a) and 3.15(b). As was the case with the Repetitive Load-Release tests, noticeable stiffening of the Roadrain occurs with repeated load applications, which can be seen by the smaller deflections apparent in the final loading data than those of the previous two tests for each specimen.

From this data, characteristic curves were made utilizing the end-points of deflection for each load increment. The points were used to formulate polynomial trendlines, as shown in Figures 3.16(a) and 3.16(b). It should be noted that while it would appear that the first loading of Specimen C2WC057 \#1 produced much higher stiffness values than the first loading of $\mathrm{C} 2 \mathrm{WC} 04 \# 2$, this is not necessarily the case, since C2WC057 \#1 had some load applied before the Sustained loading test was conducted with that specimen; the first Sustained loading test of Specimen C2WC04 \#2 was in fact the first time that specimen was loaded, so the trendline for this test will be used to represent the first loading of Roadrain when accounting for creep effects at small stress levels. The relationship given for this is:

$$
\sigma_{\mathrm{y}}=2,800 \cdot \varepsilon_{\mathrm{y}}{ }^{2}
$$

This equation is applicable for the first application of a long-duration static load up to a stress level of $100 \mathrm{psi}$.

A comparison of the trendlines for the final Sustained loading test of both specimen shows a good correlation between stress-strain relationships of the two samples. A characteristic curve for multiple loadings of Roadrain which accounts for creep effects was developed using the average of the displacements from the final Sustained loading tests of the two specimens, as shown in Figure 3.17. This gives the following relationship:

$$
\sigma_{\mathrm{y}}=10,004 \cdot \varepsilon_{\mathrm{y}}{ }^{2}
$$


This equation is eventually applicable for the repeated application of long-duration static loads up to $100 \mathrm{psi}$.

\subsubsection{Summary and Analysis of Results}

The vertical compression tests in their various forms gave valuable characteristic equations describing the non-linear relationships between the strains that would be induced in a Roadrain layer and the types of stresses applied. In particular, both types of Load-Release tests gave stress-strain relationships for strains induced by short-term loading; the original Load-Release gave this relationship for the first time a layer of Roadrain is loaded, and the Repetitive Load-Release tests gave it for Roadrain which had already been tested multiple times. Also, the Sustained loading tests gave relationships which account for the creep effects which are inevitable when dealing with long-term static loading of this material; this testing scheme gave relationships for both the first time of loading, and for after multiple loadings. These equations were given throughout this chapter, and are summarized in Table 3.6.

Figures 3.18 and 3.19 compare the stress-strain relationships of these four characteristic equations. It can be seen that the most deformation at any given stress level occurs from the initial sustained loading of Roadrain, and the least occurs with the shortterm loading after multiple load applications. It is noted that the initial load-release trendline was developed at a much higher stress range than the other three characteristic trendlines.

In addition to the stress-strain relationships defined through the compression tests, the rebound of the Repetitive Load-Release tests gave a relationship between the Tangent Modulus of Elasticity and the stress level in the Roadrain layer. However, since the zerostress, zero-strain point in the unloading portion of the stress-strain curve is dependent upon the stress level from which the Roadrain rebounds, the stress-strain relationship cannot be characterized in the same way as the other tests. These equations may be valuable when attempting to create more advanced models the material behavior though, since their slopes give rebound stiffnesses of the material as stress levels decrease; many computer-based FEM analysis programs allow for the input of separate loading and 
unloading stiffness parameters of a non-linear material at various stress levels (Algor, Abaqus). 
Table 3.1: Mix Designs for the Two Concrete Mixes Used in Vertical Compression Test Specimens

\begin{tabular}{|l|c|c|}
\hline \multicolumn{1}{|c|}{ INGREDIENT } & \multicolumn{2}{c|}{ WEIGHT [ lb / $\left.\mathbf{y d}^{\mathbf{3}}\right]$} \\
& $\mathbf{w} / \mathbf{c}=\mathbf{0 . 4}$ & $\mathbf{w / c}=\mathbf{0 . 5 7}$ \\
\hline Coarse Aggregate (\#57 limestone) & 1611 & 1611 \\
\hline Sand & 1450 & 2181 \\
\hline Type I Portland Cement & 788 & 553 \\
\hline Water & 277 & 277 \\
\hline
\end{tabular}

Table 3.2: Loading Scenario for Each Vertical Compression Test Specimen

\begin{tabular}{|c|c|c|c|}
\hline CYLINDER & LOAD-RELEASE & $\begin{array}{c}\text { REPETITIVE } \\
\text { LOAD-RELEASE }\end{array}$ & $\begin{array}{c}\text { SUSTAINED } \\
\text { LOADING }\end{array}$ \\
\hline C1WC04 \#1 & $\mathrm{X}$ & & \\
\hline C1WC04 \#2 & $\mathrm{X}$ & & \\
\hline C1WC04 \#3 & $\mathrm{X}$ & & \\
\hline C1WC057 \#1 & $\mathrm{X}$ & & \\
\hline C1WC057 \#2 & $\mathrm{X}$ & & \\
\hline C1WC057 \#3 & $\mathrm{X}$ & $\mathrm{X} \quad(\mathrm{x} 2)$ & \\
\hline C2WC04 \#1 & & & $\mathrm{X}$ \\
\hline C2WC04 \#2 & & $\mathrm{X}$ & \\
\hline C2WC04 \#3 & & $\mathrm{X}$ & \\
\hline C2WC057 \#1 & & $\mathrm{X}$ & \\
\hline C2WC057 \#2 & & & \\
\hline C2WC057 \#3 & & & \\
\hline
\end{tabular}


Table 3.3: Target Loads for Each Load-Release Compressive Test Specimen

\begin{tabular}{|c|c|c|}
\hline CYLINDER & $\begin{array}{c}\text { TARGET LOAD } \\
{[\mathrm{kips}]}\end{array}$ & $\begin{array}{c}\text { COMPRESSIVE STRENGTH } \\
{[\mathrm{kips}]}\end{array}$ \\
\hline C1WC04 \#1 & 50 & 184.4 \\
\hline C1WC04 \#2 & 110 & --- \\
\hline C1WC04 \#3 & 110 & --- \\
\hline C1WC057 \#1 & 105 & 127.6 \\
\hline C1WC057 \#2 & 86 & --- \\
\hline C1WC057 \#3 & 90 & --- \\
\hline
\end{tabular}


Table 3.4: Load Patterns for Repetitive Load-Release Tests

\begin{tabular}{|c|c|}
\hline CYLINDER & LOAD-RELEASE PATTERN [All Loads in lbs] \\
\hline C2WC04 \#1 & $(0 \rightarrow 250 \rightarrow 0)[\mathrm{x} 4] \rightarrow(0 \rightarrow 500 \rightarrow 0)[\mathrm{x} 3] \rightarrow(0 \rightarrow 1000 \rightarrow 0)[\mathrm{x} 3] \rightarrow(0 \rightarrow 1500 \rightarrow 0)[\mathrm{x} 4] \rightarrow(0 \rightarrow 3000 \rightarrow 0)[\mathrm{x} 3]$ \\
\hline C2WC04 \#1b & $(0 \rightarrow 500 \rightarrow 0) \rightarrow(0 \rightarrow 1000 \rightarrow 0) \rightarrow(0 \rightarrow 250 \rightarrow 0) \rightarrow(0 \rightarrow 1500 \rightarrow 0) \rightarrow(0 \rightarrow 3000 \rightarrow 0)$ \\
\hline C2WC04 \#3 & $(0 \rightarrow 250 \rightarrow 0)[\mathrm{x} 4] \rightarrow(0 \rightarrow 500 \rightarrow 0)[\mathrm{x} 3] \rightarrow(0 \rightarrow 1000 \rightarrow 0)[\mathrm{x} 3] \rightarrow(0 \rightarrow 1500 \rightarrow 0)[\mathrm{x} 2] \rightarrow(0 \rightarrow 3000 \rightarrow 0)[\mathrm{x} 3]$ \\
\hline C2WC057 \#2 & $(0 \rightarrow 1500 \rightarrow 0) \rightarrow(0 \rightarrow 2500 \rightarrow 0) \rightarrow(0 \rightarrow 1500 \rightarrow 0) \rightarrow(0 \rightarrow 2500 \rightarrow 0) \rightarrow(0 \rightarrow 4000 \rightarrow 0)$ \\
\hline C2WC057 \#3 & $(0 \rightarrow 250 \rightarrow 0)[\mathrm{x} 3] \rightarrow(0 \rightarrow 500 \rightarrow 0)[\mathrm{x} 3] \rightarrow(0 \rightarrow 1000 \rightarrow 0)[\mathrm{x} 3] \rightarrow(0 \rightarrow 1500 \rightarrow 0)[\mathrm{x} 3] \rightarrow(0 \rightarrow 3000 \rightarrow 0)[\mathrm{x} 3]$ \\
\hline
\end{tabular}

Table 3.5: Load Patterns for Sustained Loading Tests

\begin{tabular}{|c|c|}
\hline CYLINDER & SUSTAINED LOADING PATTERN [All Loads in lbs] \\
\hline C2WC04 \#2 & $(0 \rightarrow 200 \rightarrow 400 \rightarrow 600 \rightarrow 800 \rightarrow 1000 \rightarrow 1500 \rightarrow 2000 \rightarrow 3000 \rightarrow 0)[\mathrm{x} 3]$ \\
\hline C2WC057 \#1 & $(0 \rightarrow 200 \rightarrow 400 \rightarrow 600 \rightarrow 800 \rightarrow 1000 \rightarrow 1500 \rightarrow 2000 \rightarrow 3000 \rightarrow 0)[\mathrm{x} 3]$ \\
\hline
\end{tabular}


Table 3.6: Summary of Stress-Strain Relationships for Roadrain as Observed in Vertical Compression Tests

\begin{tabular}{|c|c|c|c|c|}
\hline $\begin{array}{c}\text { TESTING } \\
\text { SCHEME }\end{array}$ & $\begin{array}{c}\text { STRESS-STRAIN } \\
\text { RELATIONSHIP }\end{array}$ & $\begin{array}{c}\text { VALID STRESS } \\
\text { RANGE }\end{array}$ & $\begin{array}{c}\text { LOAD } \\
\text { HISTORY }\end{array}$ & $\begin{array}{c}\text { LOAD } \\
\text { DURATION }\end{array}$ \\
\hline Load-Release & $\sigma_{\mathrm{y}}=5,114 \cdot \varepsilon_{\mathrm{y}}{ }^{2}+234 \cdot \varepsilon_{\mathrm{y}}$ & $<2,000 \mathrm{psi}$ & Initial & Short-term \\
\hline $\begin{array}{c}\text { Repetitive } \\
\text { Load-Release }\end{array}$ & $\sigma_{\mathrm{y}}=12,156 \cdot \varepsilon_{\mathrm{y}}{ }^{2}$ & $<100 \mathrm{psi}$ & $\begin{array}{c}\text { Multiple } \\
\text { Applications }\end{array}$ & Short-term \\
\hline $\begin{array}{c}\text { Sustained Loading } \\
\sigma_{\mathrm{y}}=2,800 \cdot \varepsilon_{\mathrm{y}}{ }^{2}\end{array}$ & $<100 \mathrm{psi}$ & Initial & Long-Term \\
\hline Sustained Loading & $\sigma_{\mathrm{y}}=10,004 \cdot \varepsilon_{\mathrm{y}}{ }^{2}$ & $<100 \mathrm{psi}$ & $\begin{array}{c}\text { Multiple } \\
\text { Applications }\end{array}$ & Long-Term \\
\hline
\end{tabular}




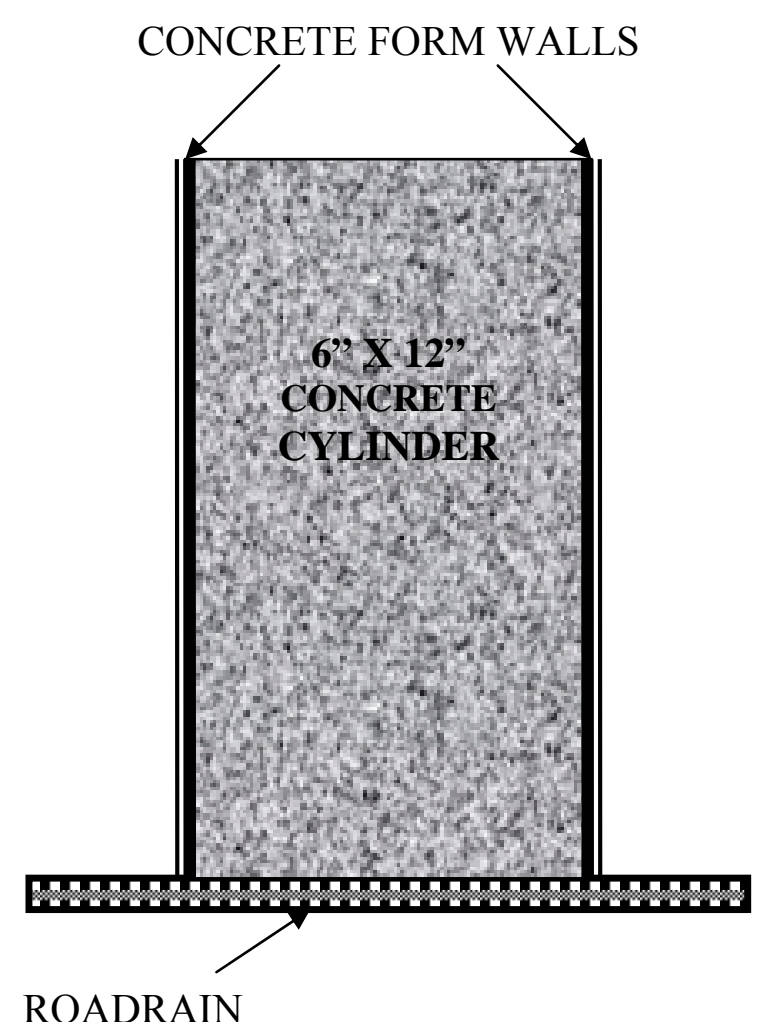

(a)

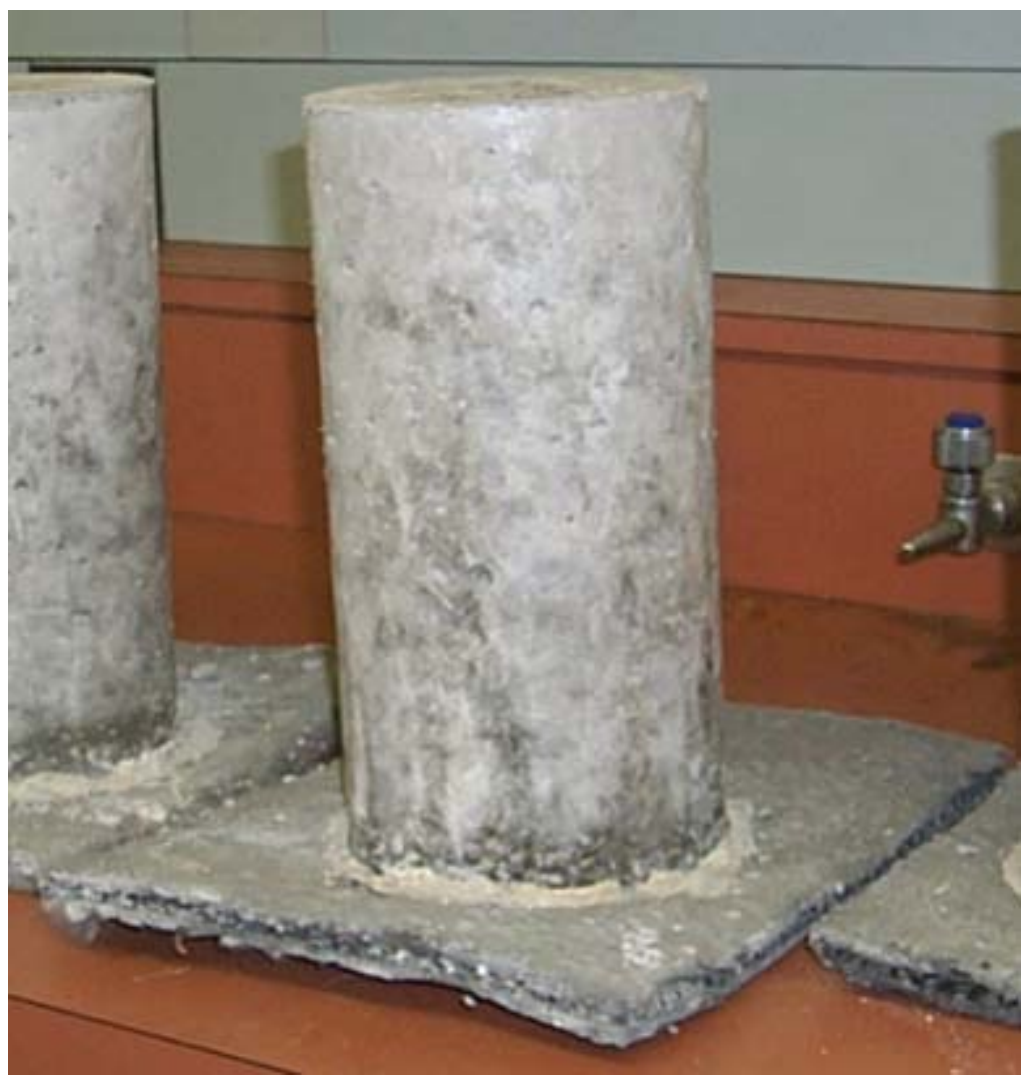

(b)

Figure 3.1: (a) Schematic Diagram, and (b) Picture of Vertical Compression Test Specimens 


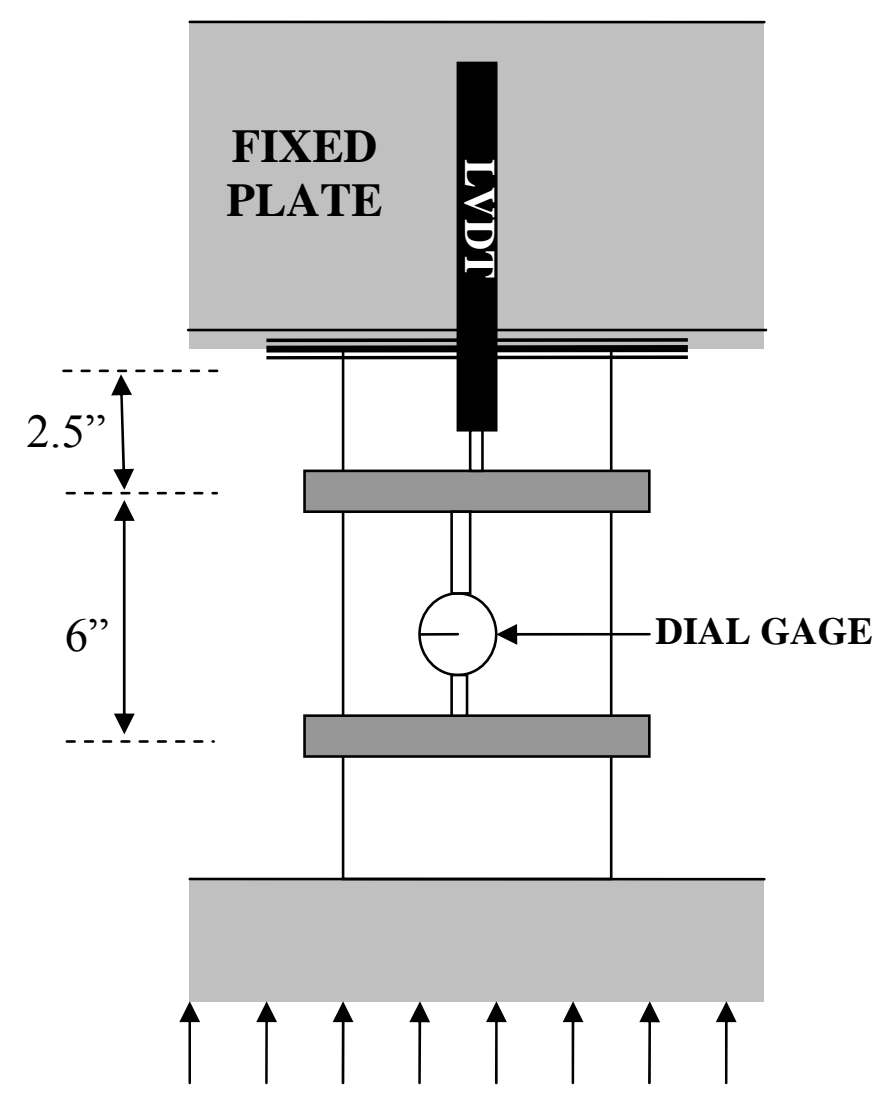

(a)

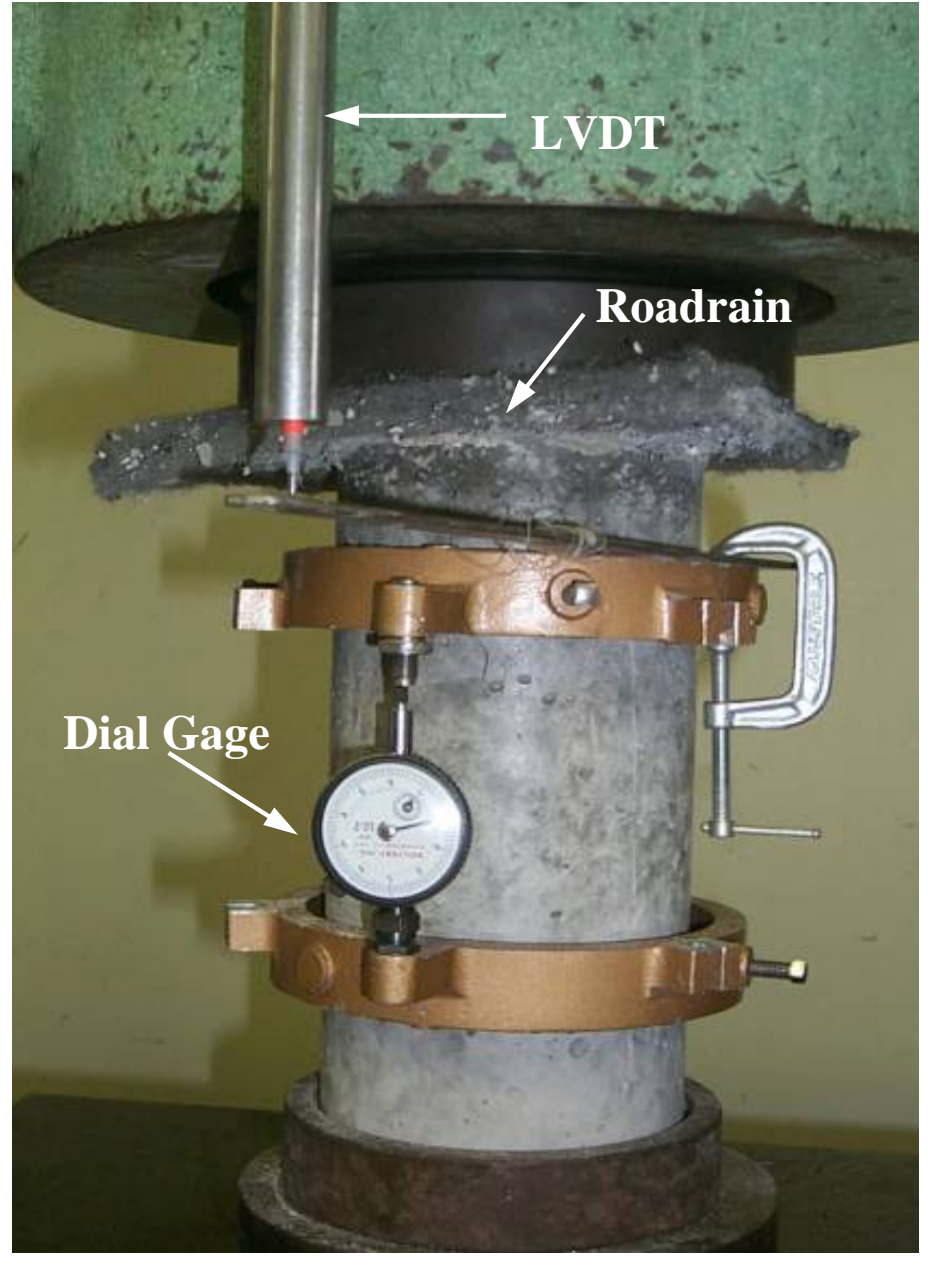

(b)

Figure 3.2: (a) Schematic Diagram, and (b) Picture of Batch \#1 Vertical Compression Test Specimens and Instrumentation 


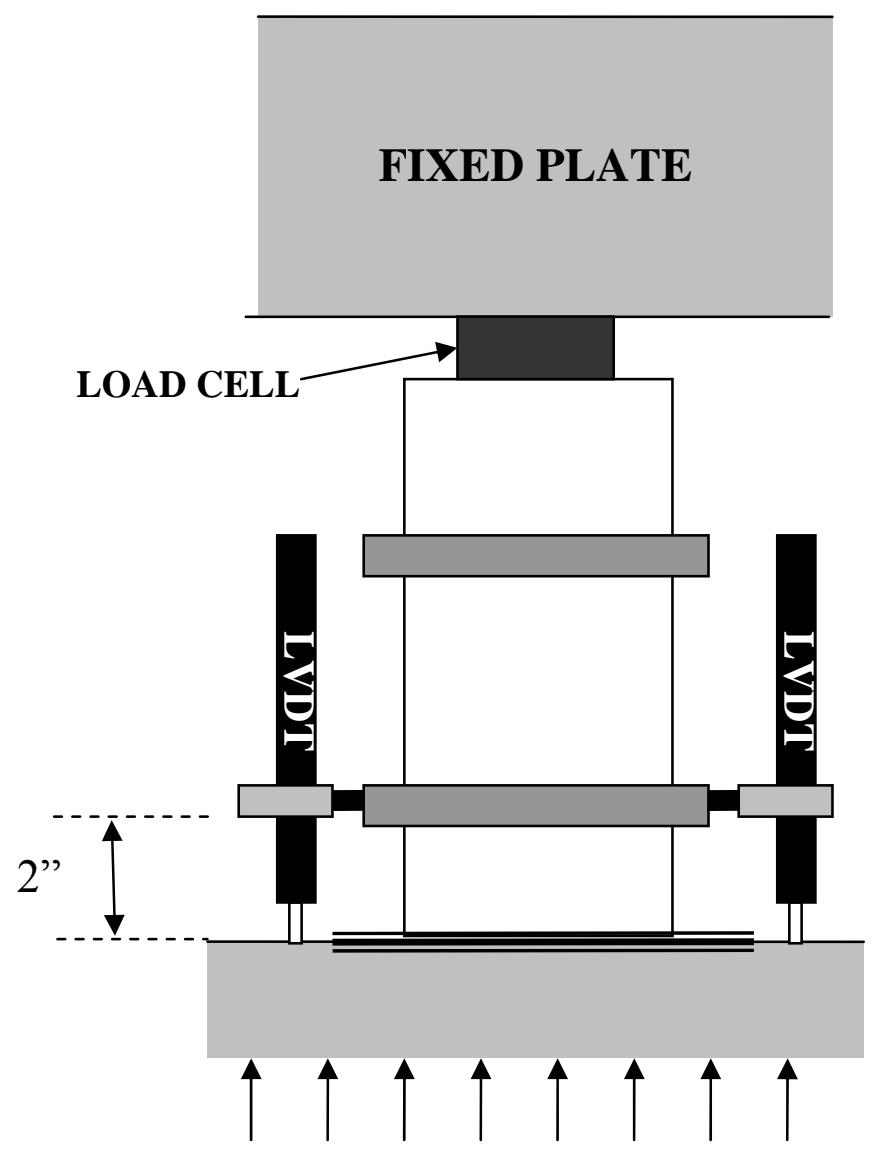

(a)

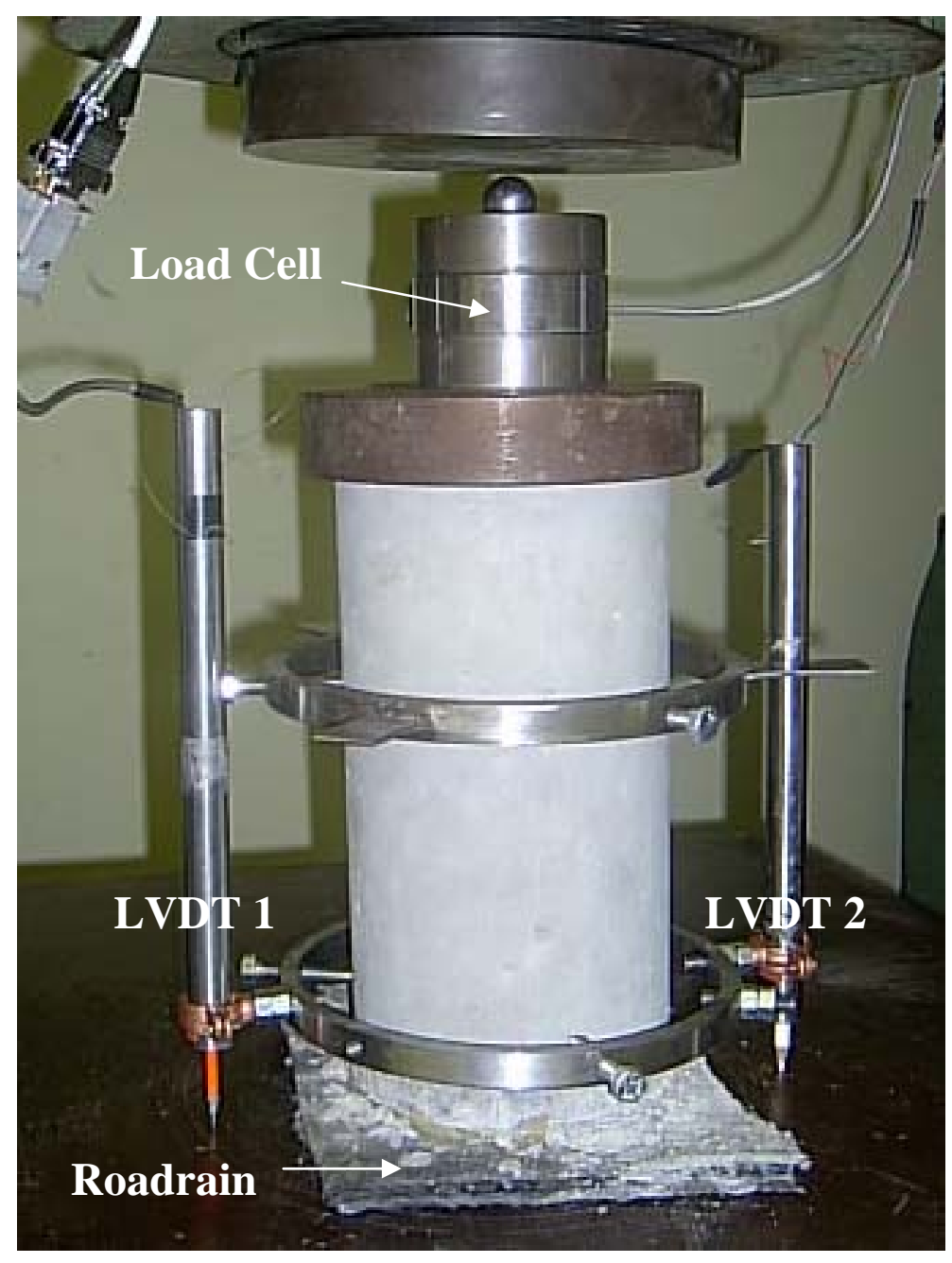

(b)

Figure 3.3: (a) Schematic Diagram, and (b) Picture of Batch \#2Vertical Compression Test Specimens and Instrumentation 




Figure 3.4: Sample Picture. Batch \#1 Vertical Compressive Test Setup 


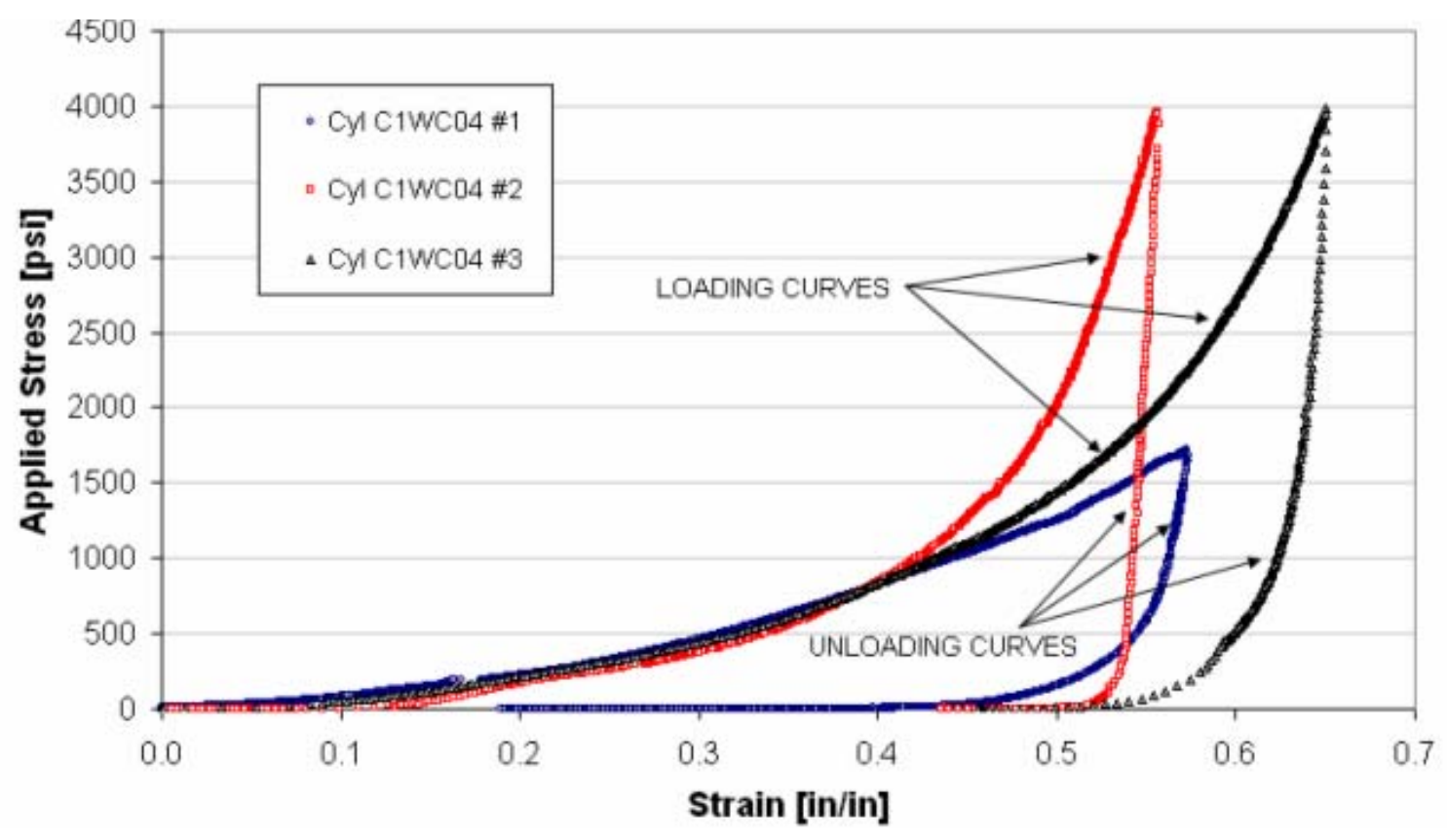

(a)

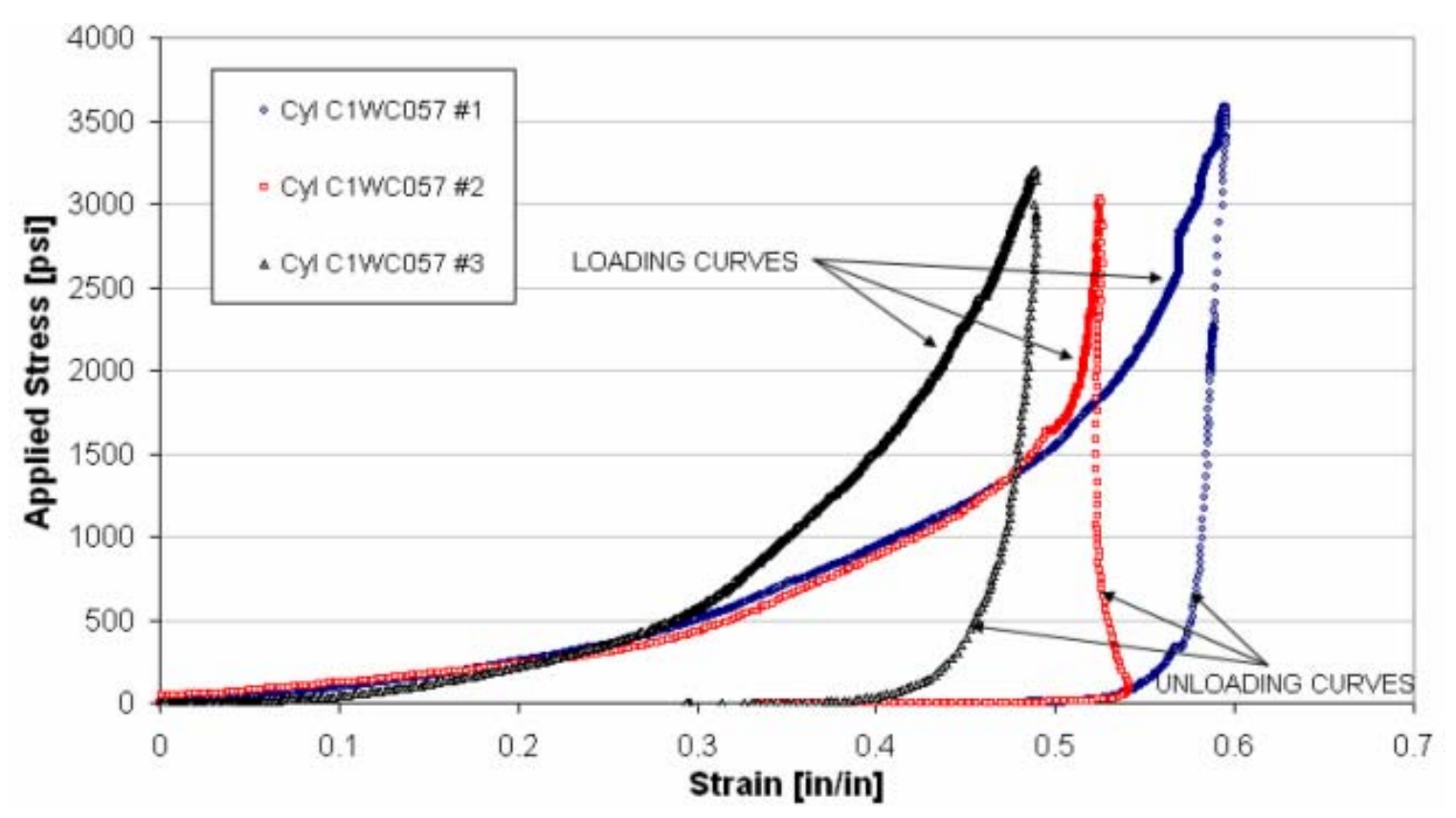

(b)

Figure 3.5: Loading and Unloading Curves for Roadrain from Load-Release Tests:

(a) All $w / c=0.4$, (b) All $w / c=0.57$. 


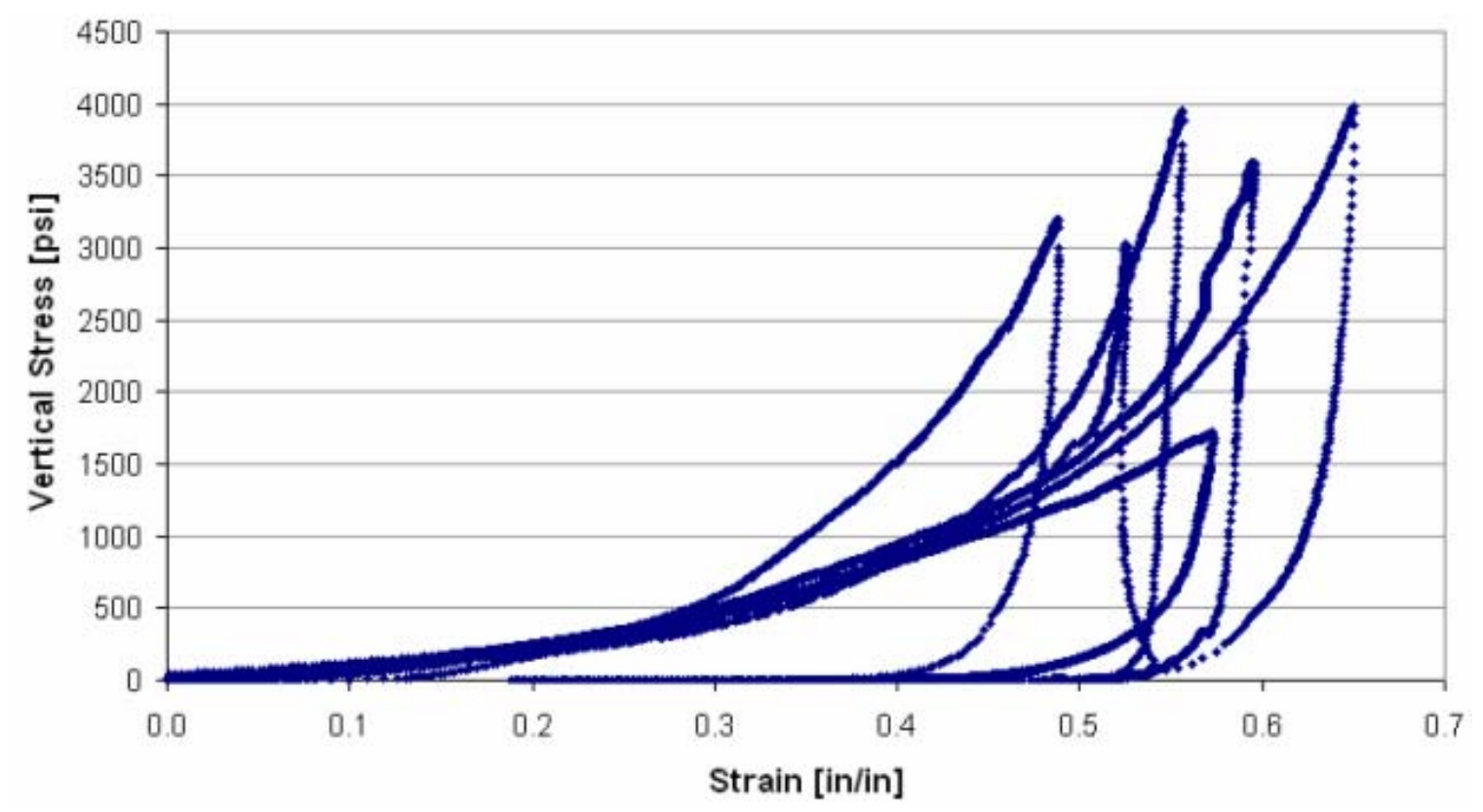

Figure 3.6: Stress-Strain Curves from Load-Release Testing of All Batch \#1 Specimens

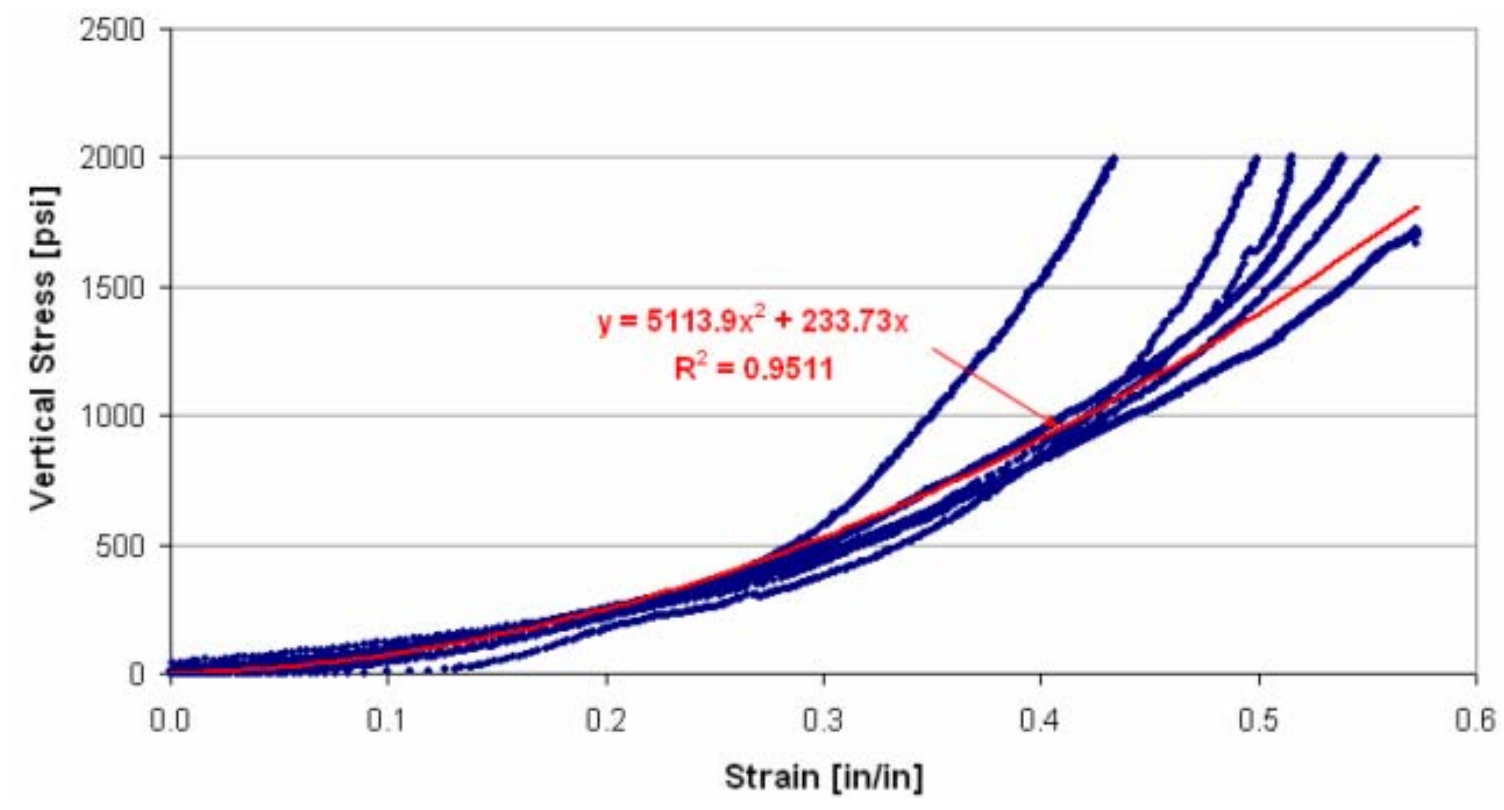

Figure 3.7: Characteristic Curve for Roadrain Based on Load Portion of Load-Release Results 


\section{Stress - Strain Curves \\ Repetitive Load-Release Test of Cylinder C2WC04 \#1 \\ Measured Deflection of Roadrain Plus 2 Inches Concrete}

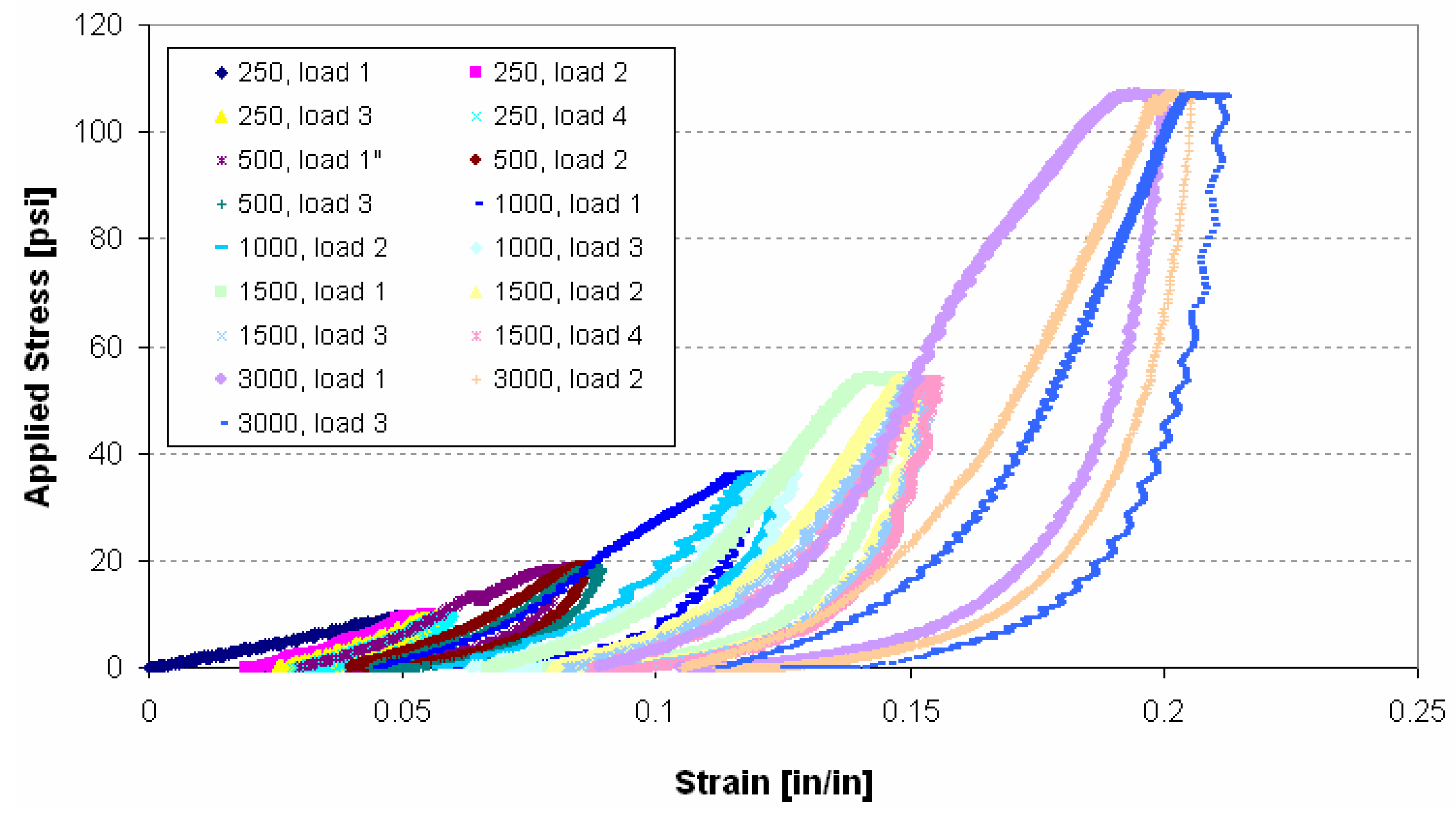

Figure 3.8: Sample Stress-Strain Data Collected from the Repetitive Load-Release Tests

(Data from Cylinder C2WC04 \#1) 
Figure 3.9

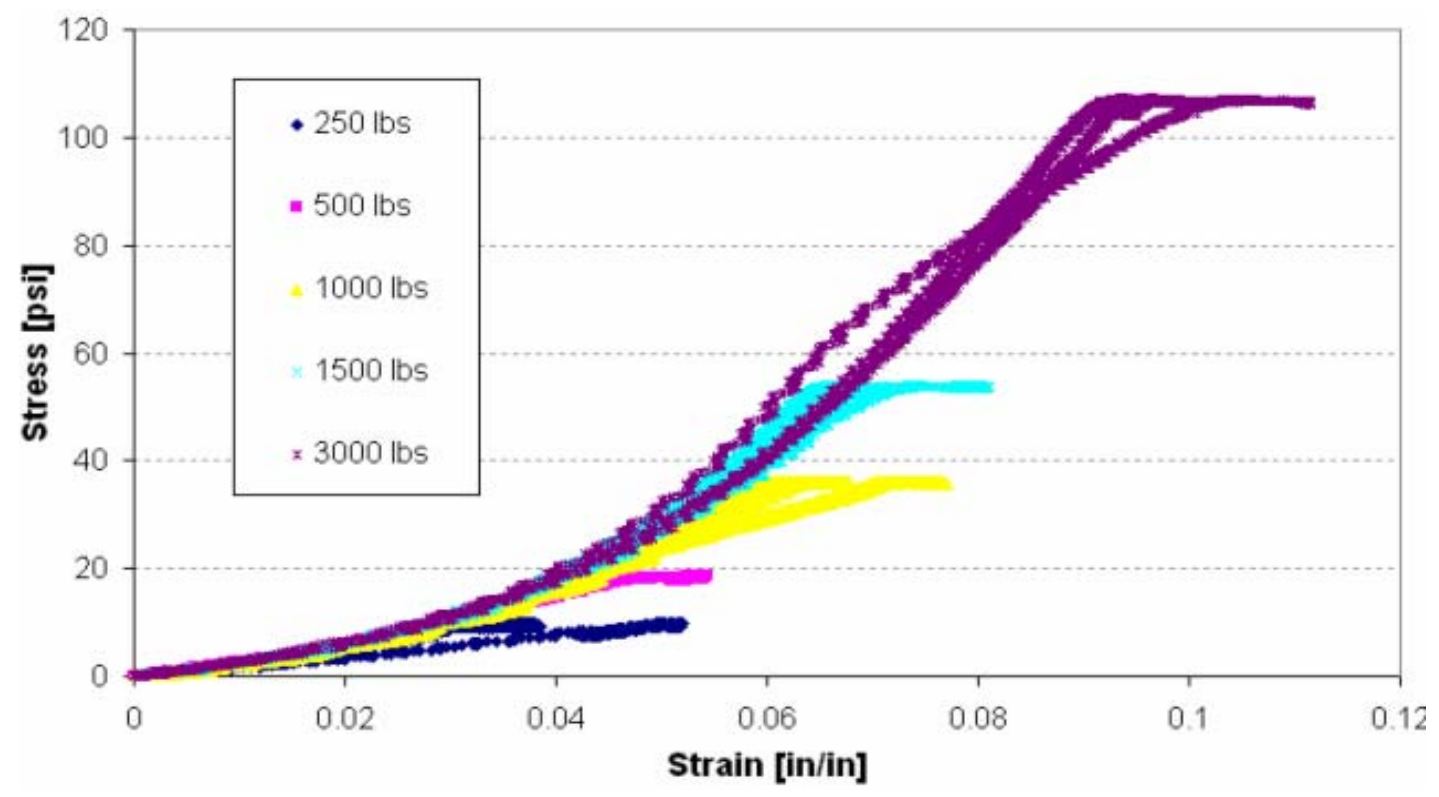

(a)

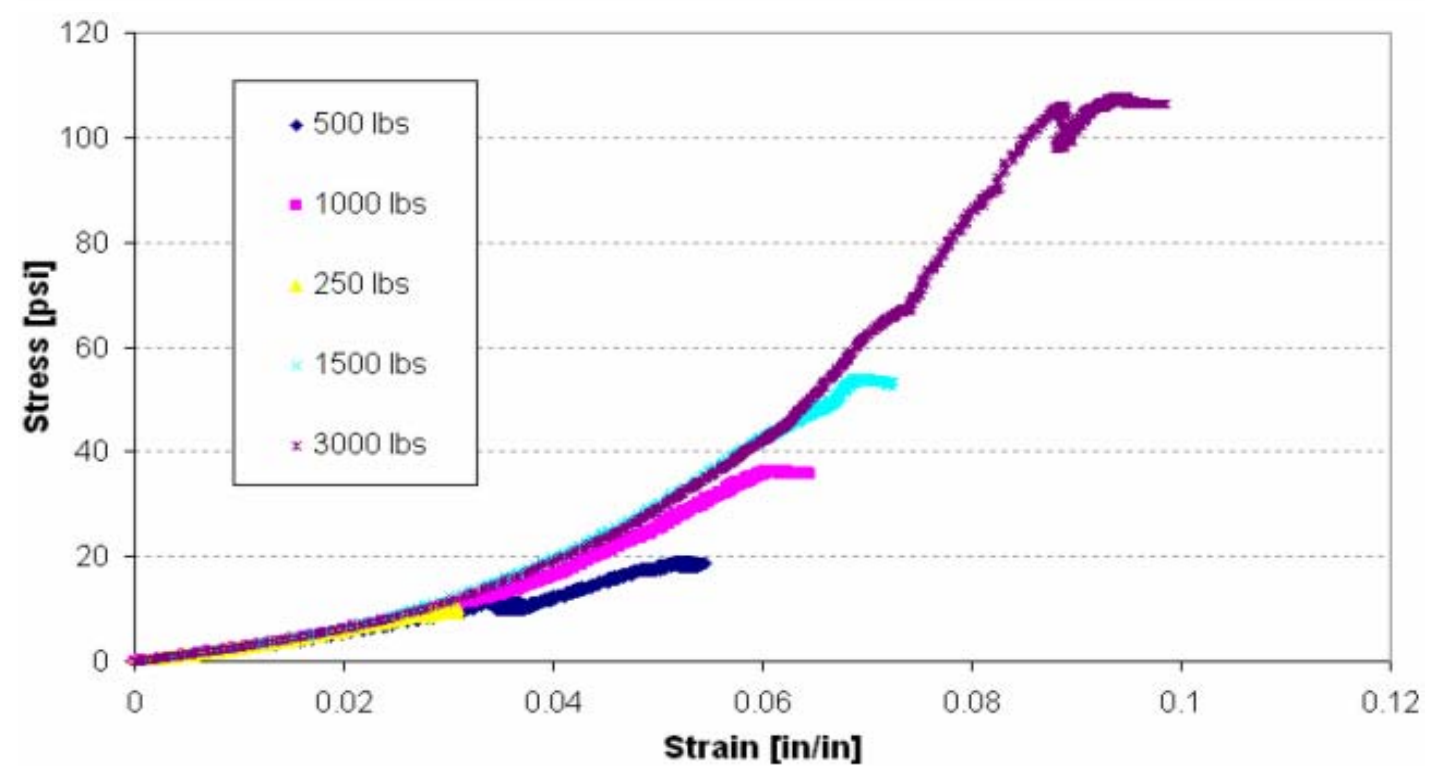

(b)

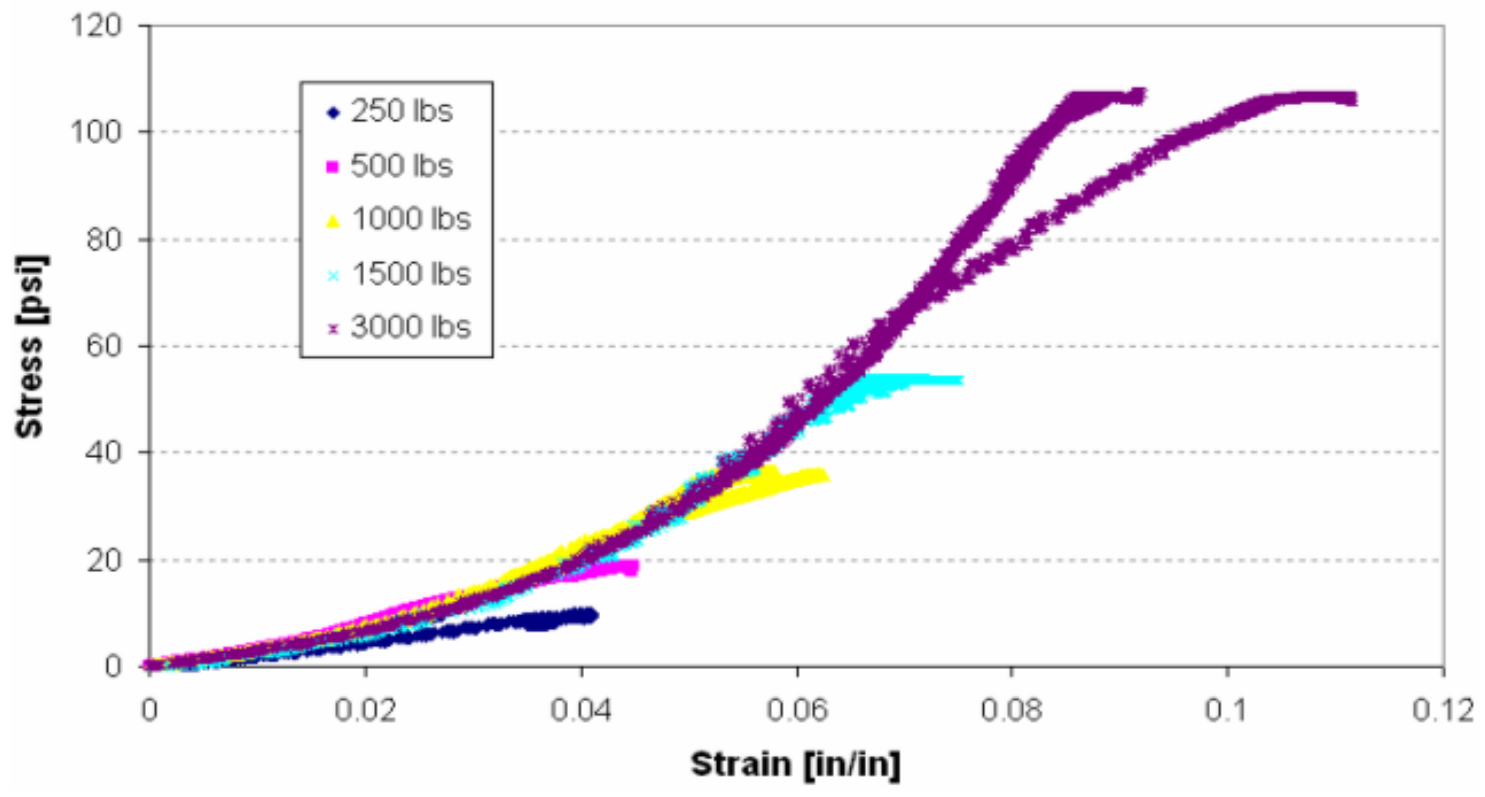

(c) 


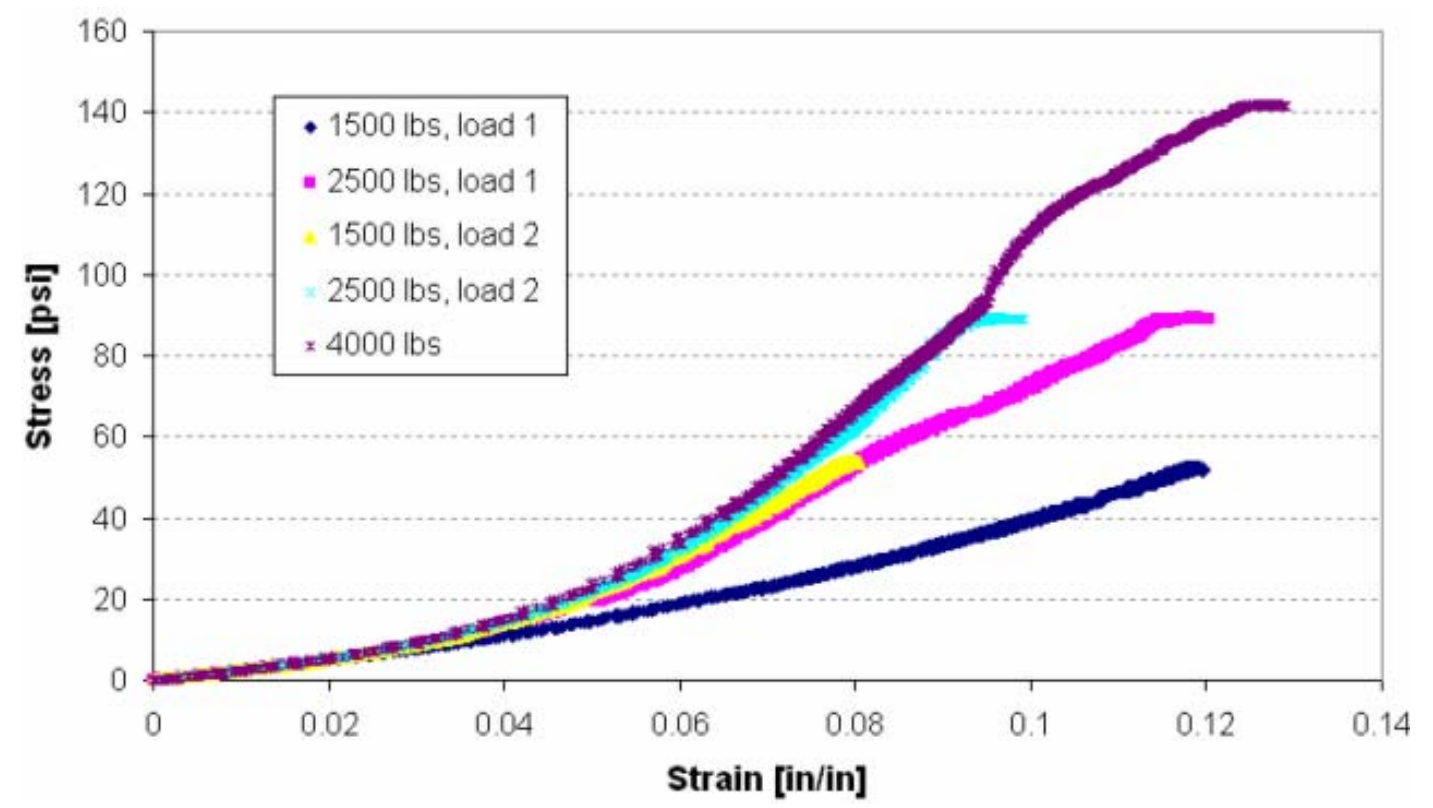

(d)

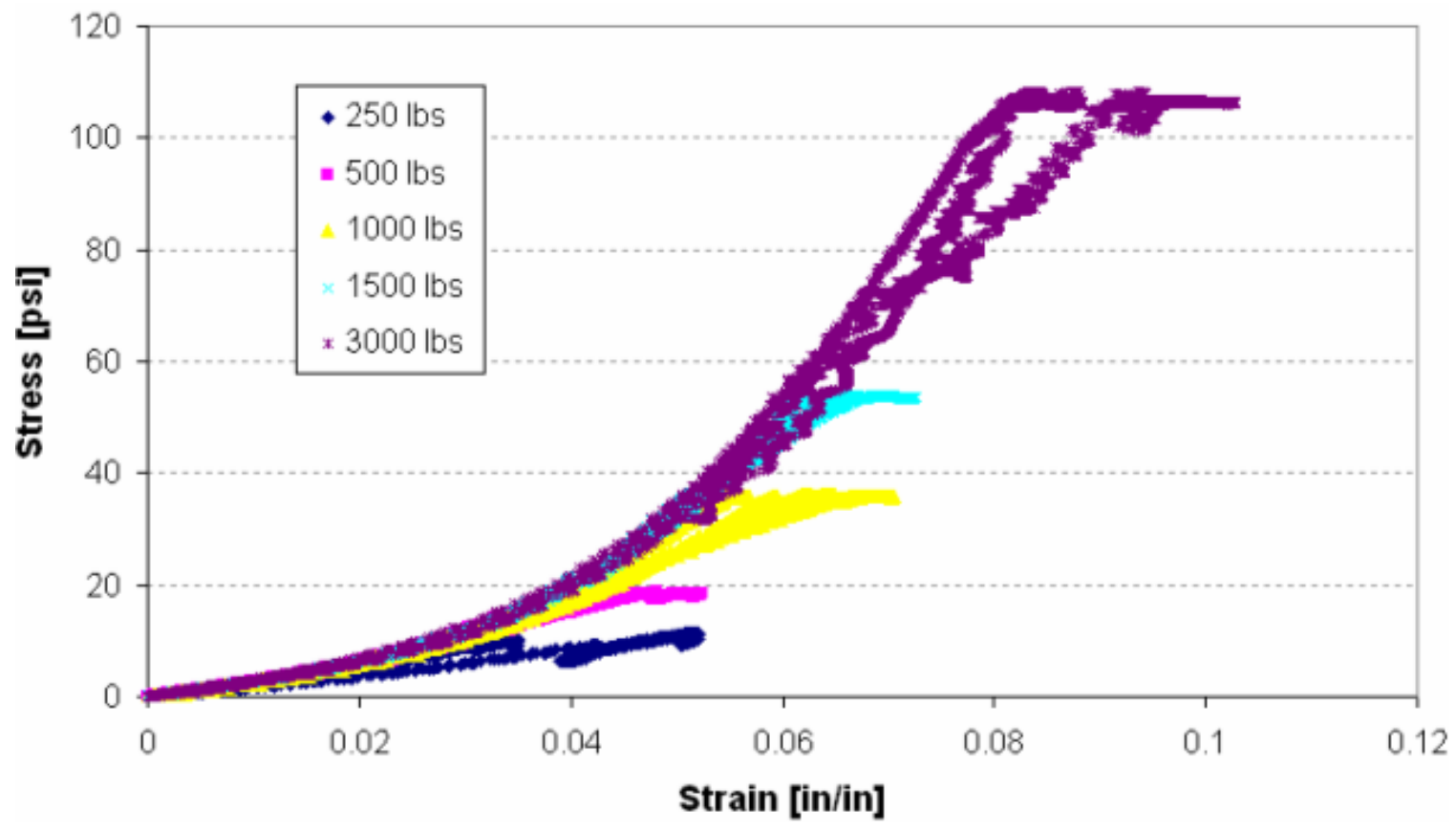

(e)

Figure 3.9: Stress-Strain Diagrams of the Loading portions of Repetitive Load-Release Tests of Cylinders (a) C2WC04 \#1, (b) C2WC04 \#1b, (c) C2WC04 \#3, (d) C2WC057 \#2, and (e) C2WC057 \#3. 


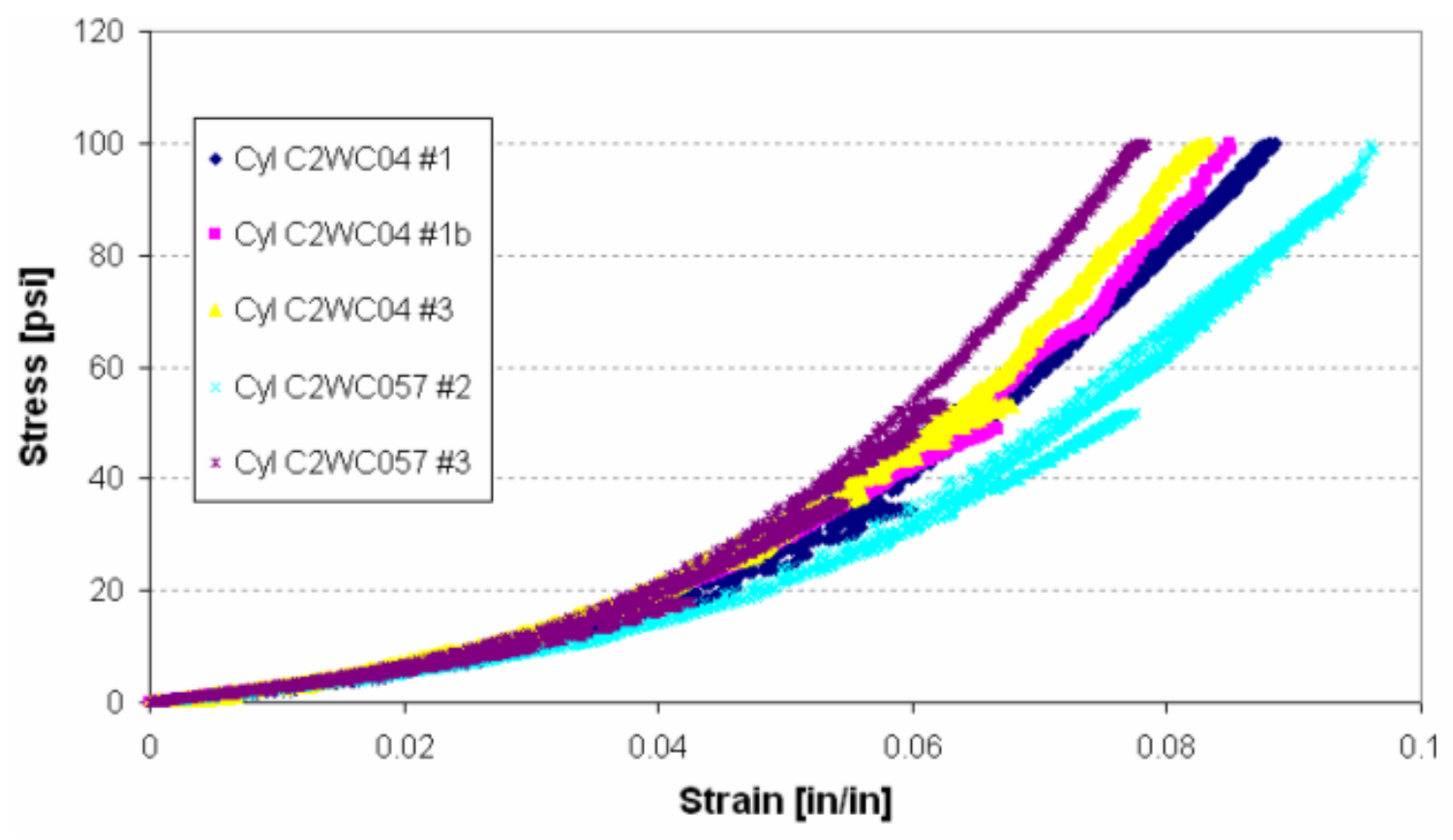

(a)

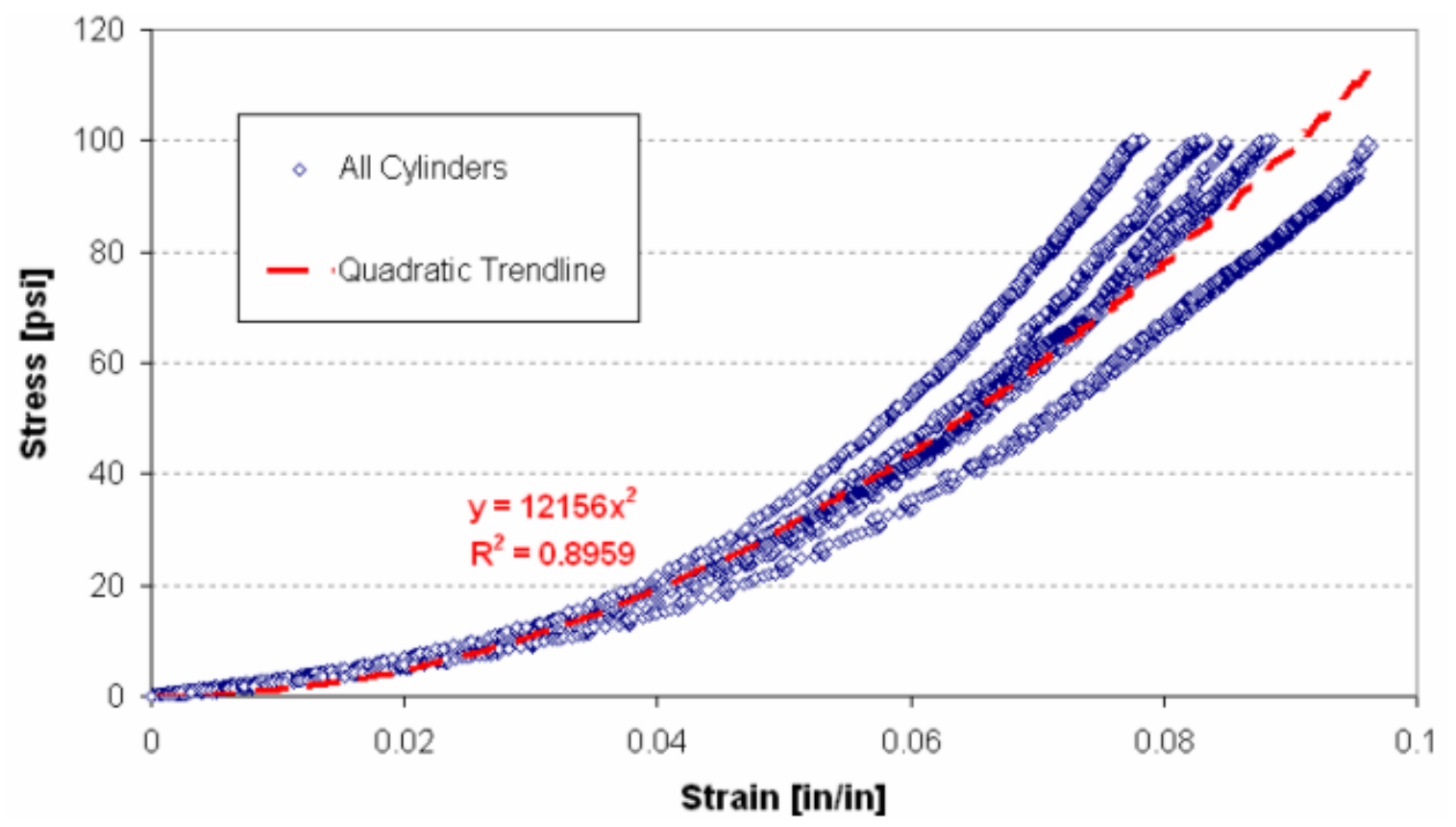

(b)

Figure 3.10: (a) Comparison of Final Loadings of Each Magnitude for All Repetitive Load-Release Test Specimens; (b) Best-Fit Characteristic Curve from Final Loadings 


\section{STRESS - STRAIN CURVES \\ Vertical Compression Tests--Unloading Curves, Cyl C2WC04 \#1 (positive strain is indicative of strain recovery upon load release)}

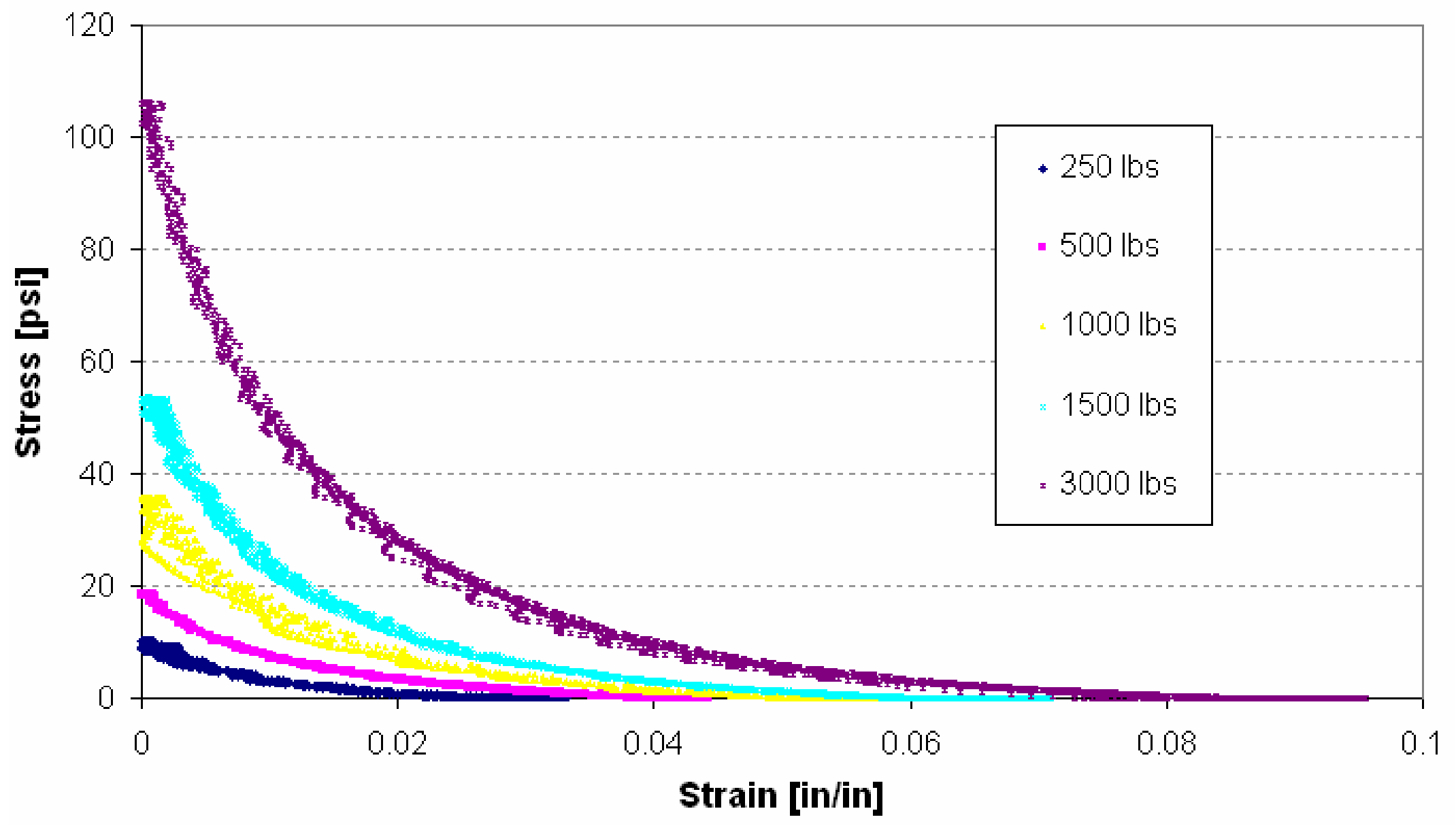

Figure 3.11: Sample Stress-Strain Curves Collected from the Unloading Portion of the Repetitive Load-Release Tests (Data from Cylinder C2WC04 \#1) 
Figure 3.12

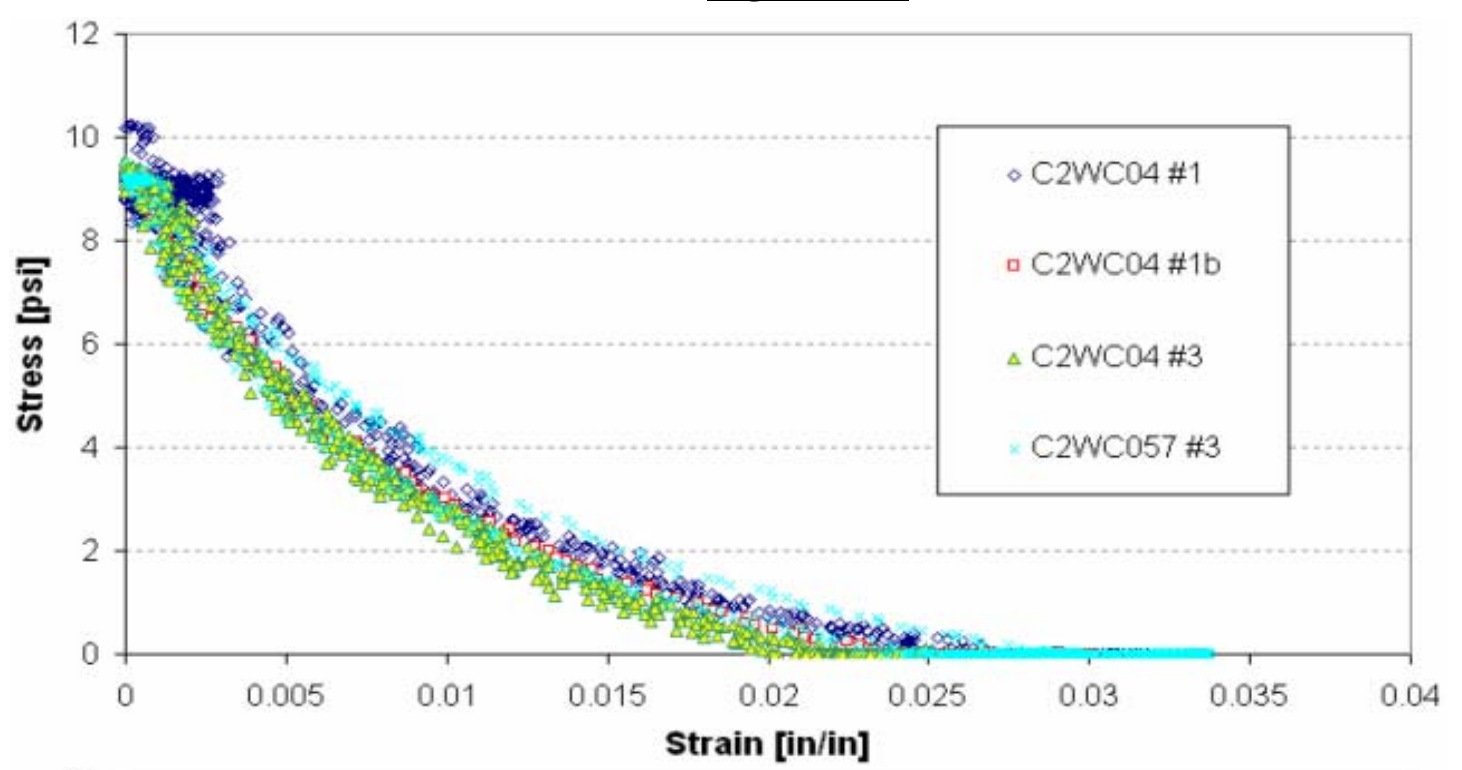

(a)

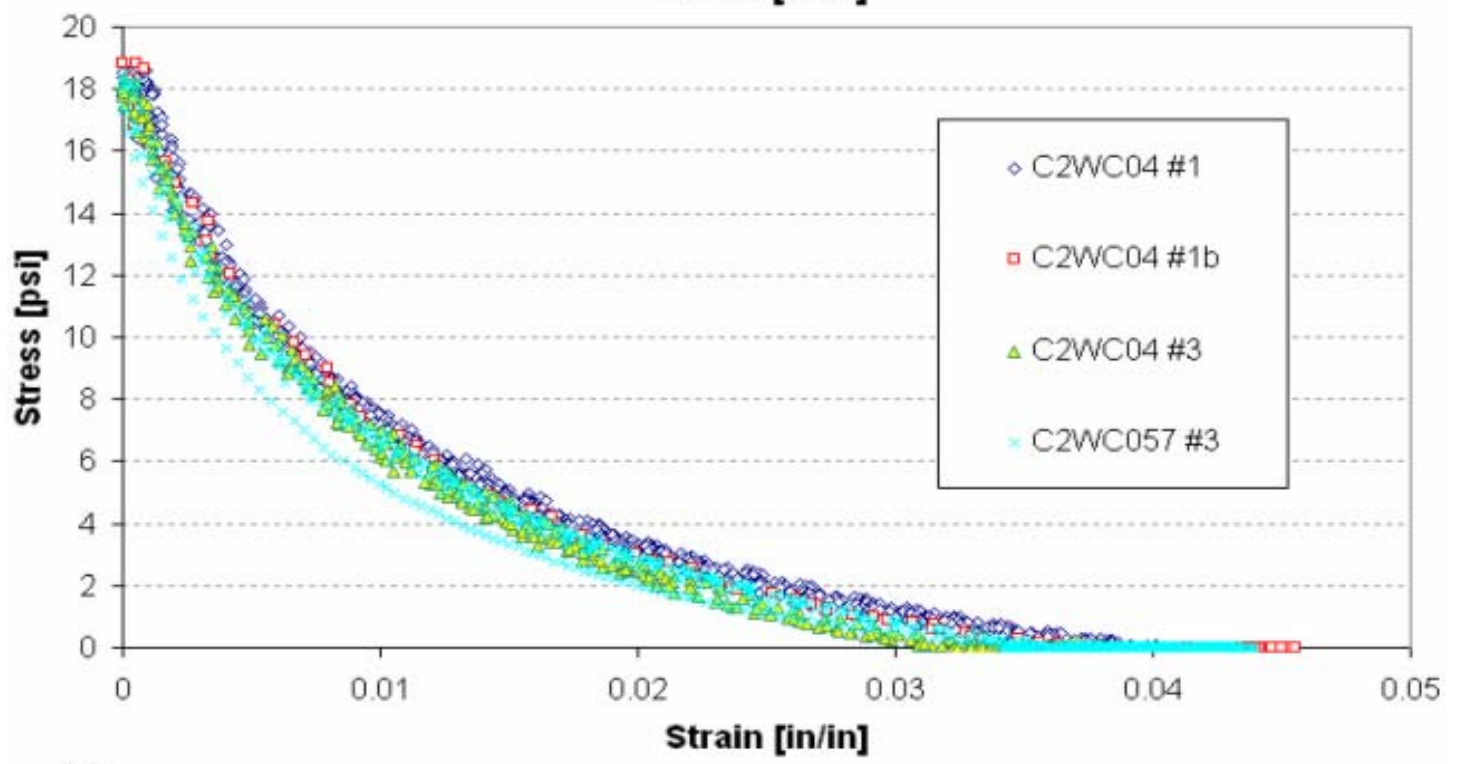

(b)

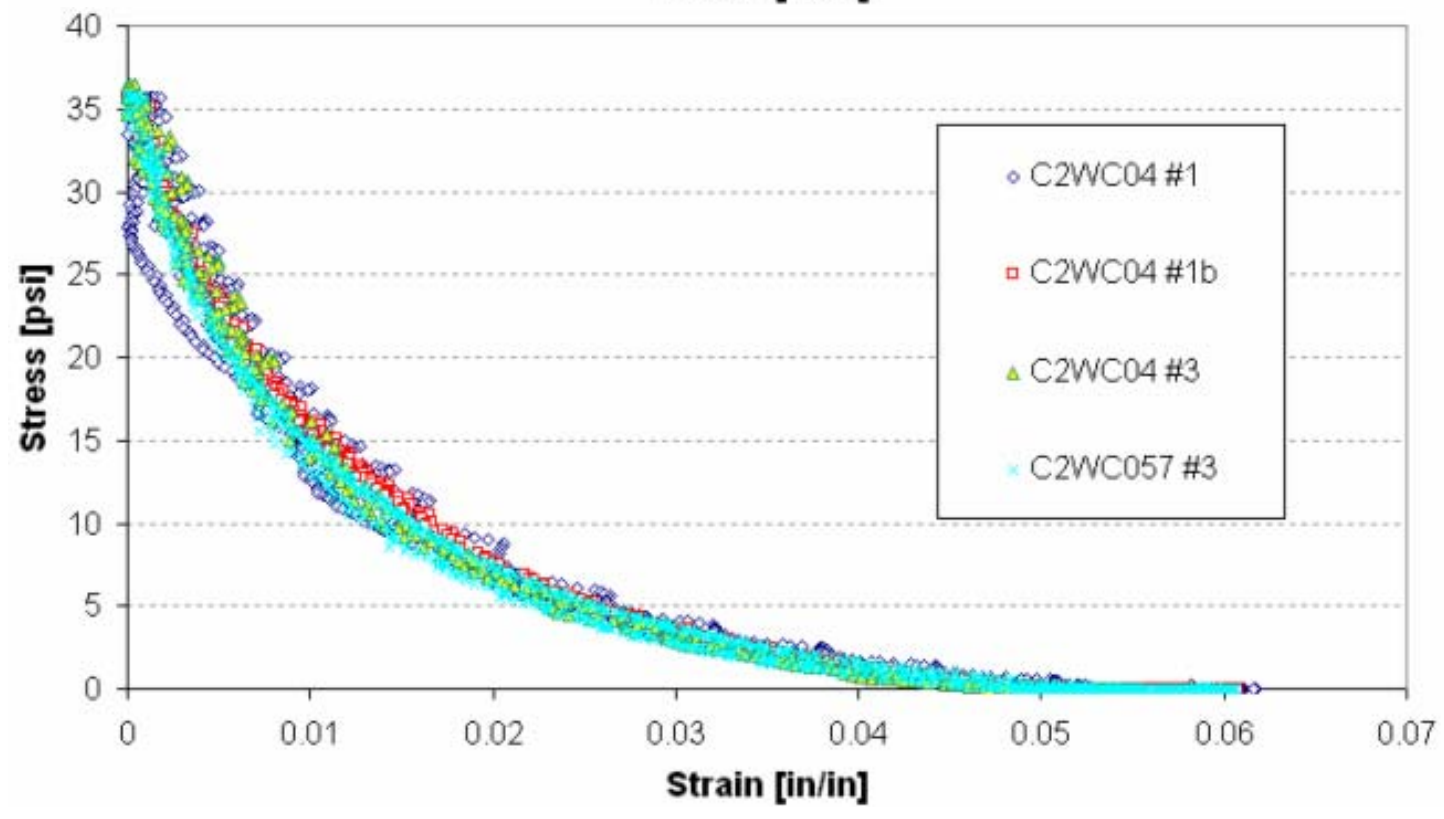

(c) 
Figure 3.12

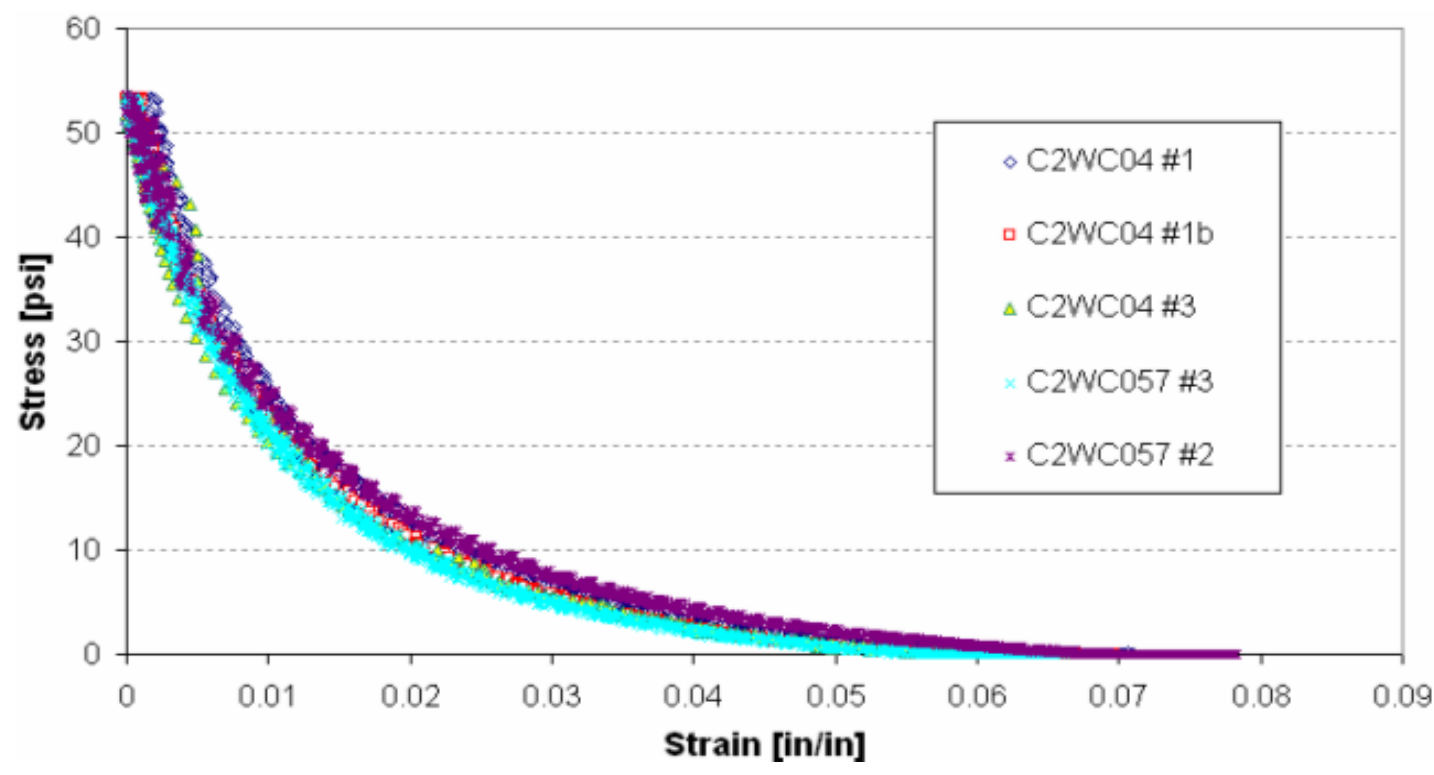

(d)

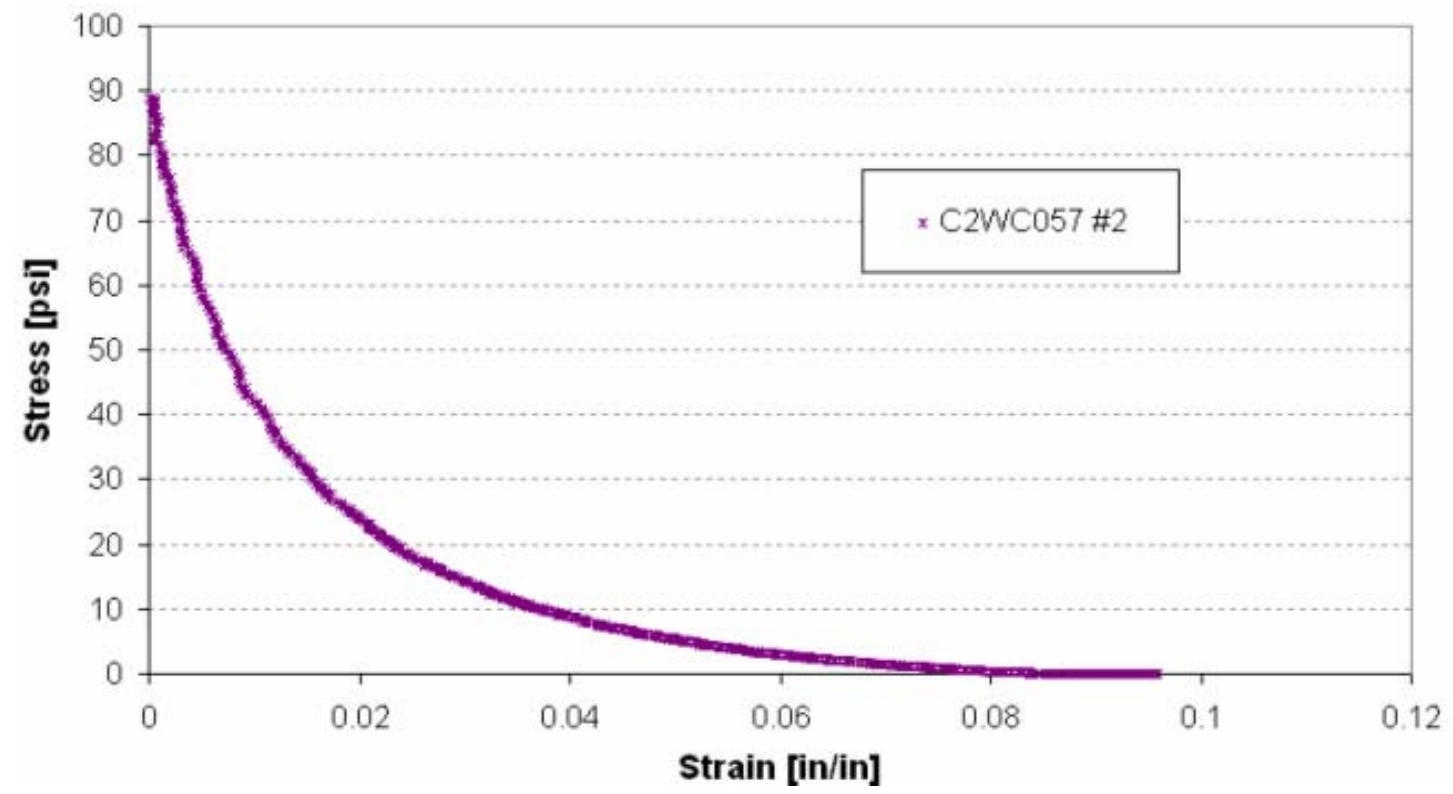

(e)

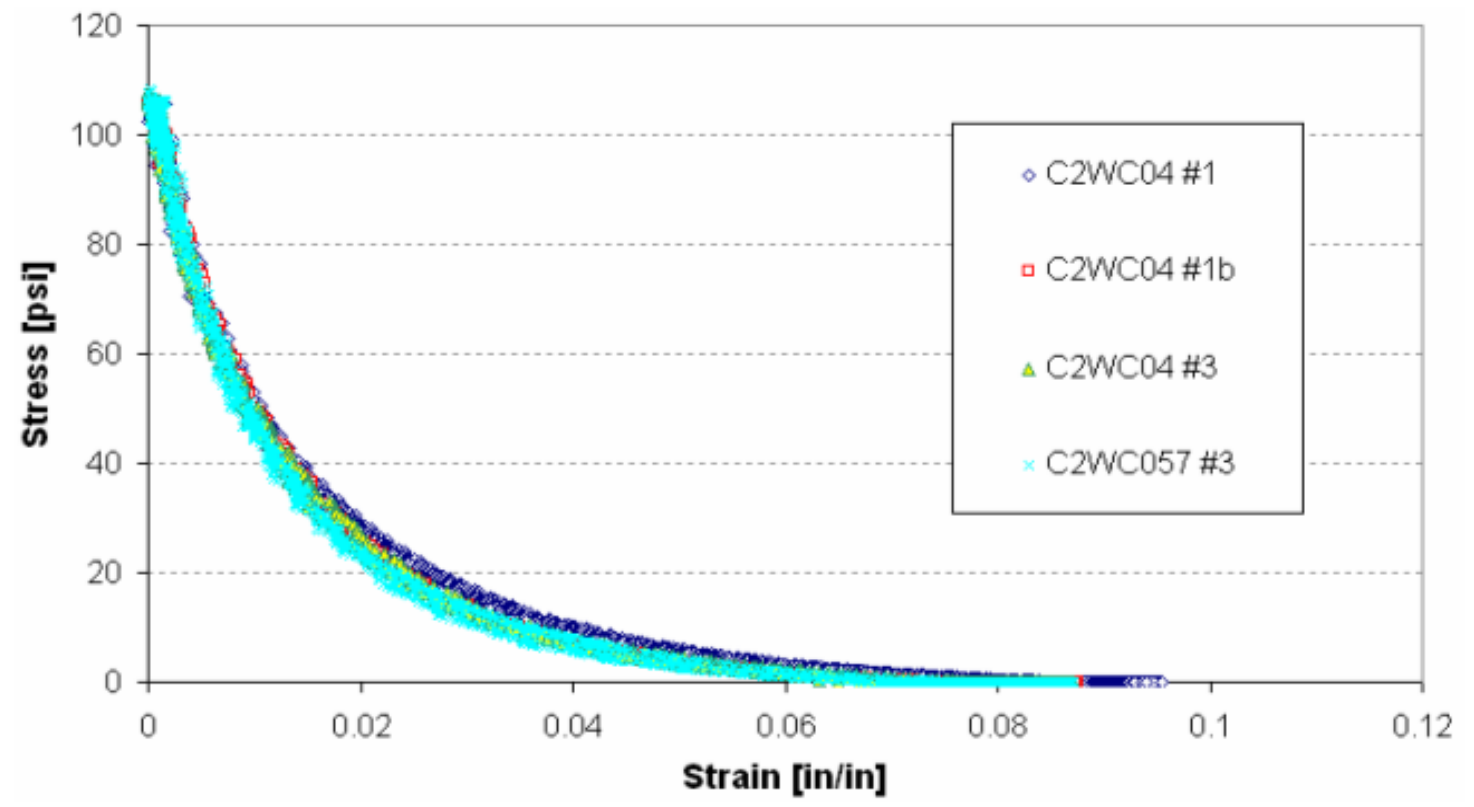

(f) 


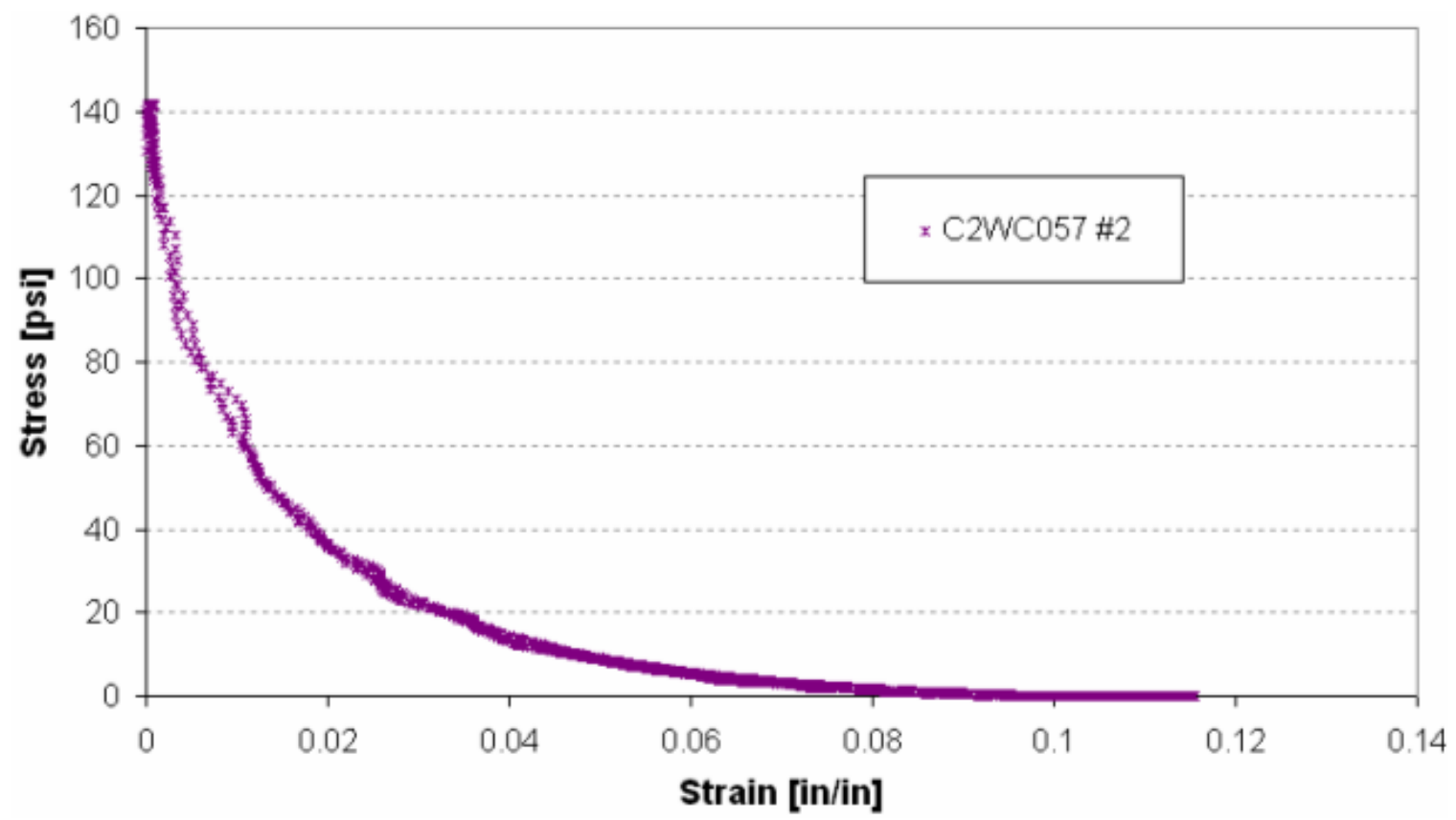

Figure 3.12: Rebound Stress-Strain Data from Release Portions of All Repetitive LoadRelease Test Specimens; Unloading from Loads of (a) $250 \mathrm{lbs}$, (b) $500 \mathrm{lbs}$, (c) $1000 \mathrm{lbs}$, (d) $1500 \mathrm{lbs}$, (e) $2500 \mathrm{lbs}$, (f) $3000 \mathrm{lbs}$, and (g) $4000 \mathrm{lbs}$ 


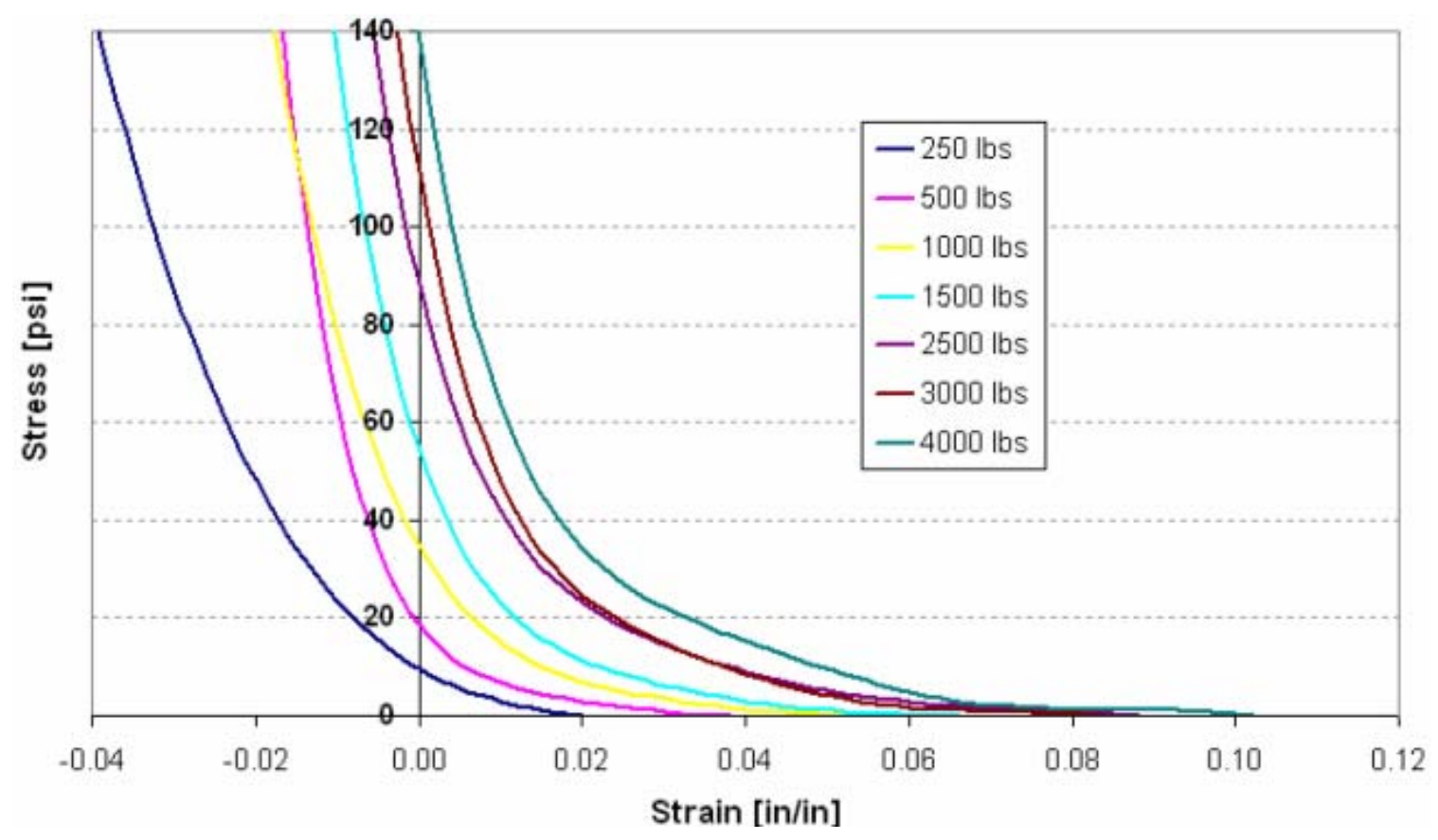

Figure 3.13: Comparison of Trendlines of Rebound Deflections from Unloading of Various Load Levels; All Repetitive Load-Release Tests

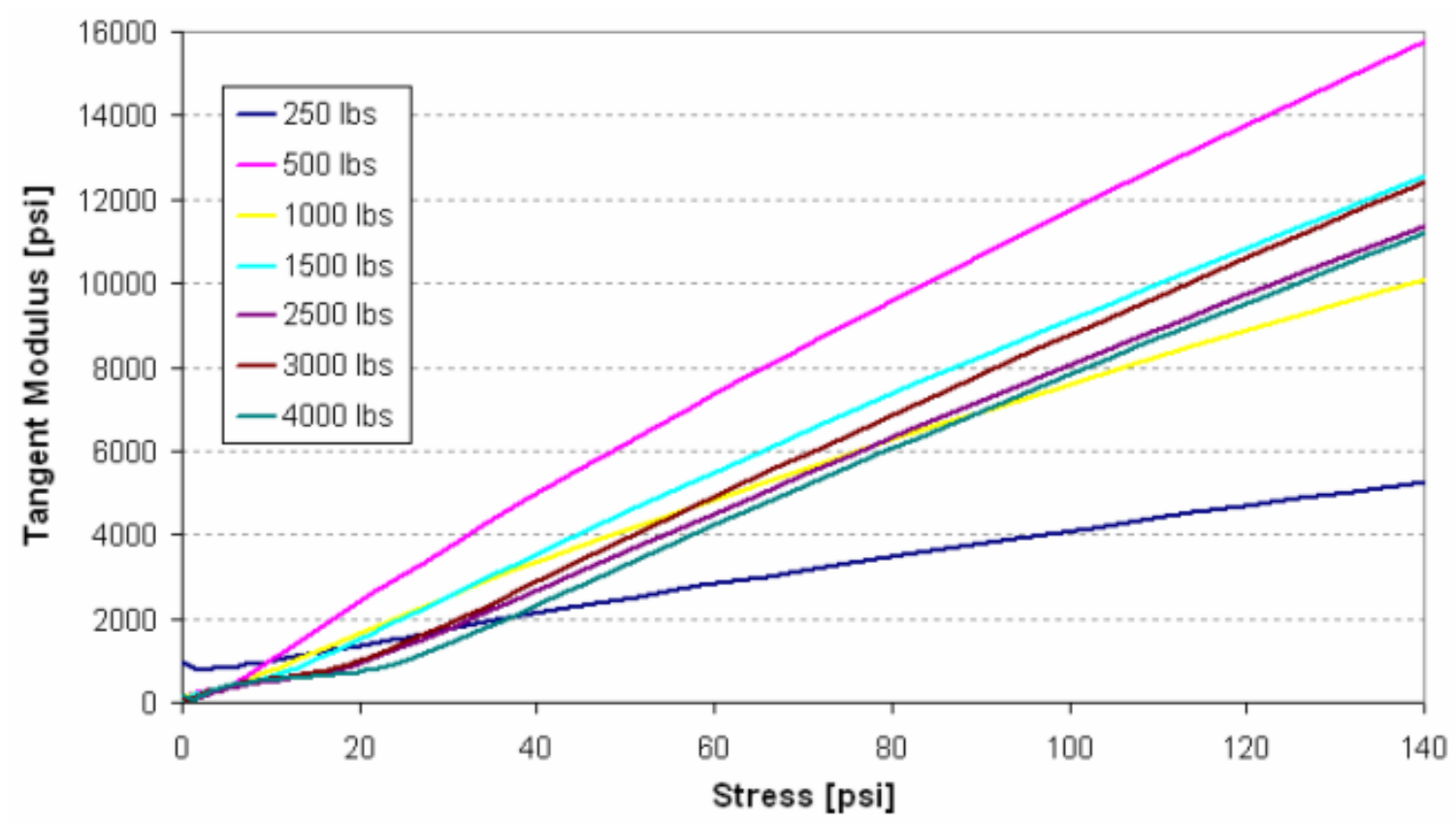

Figure 3.14: Comparison of the Slopes of the Trendlines of Rebound Deflections from Unloading of Various Load Levels; All Repetitive Load-Release Tests 


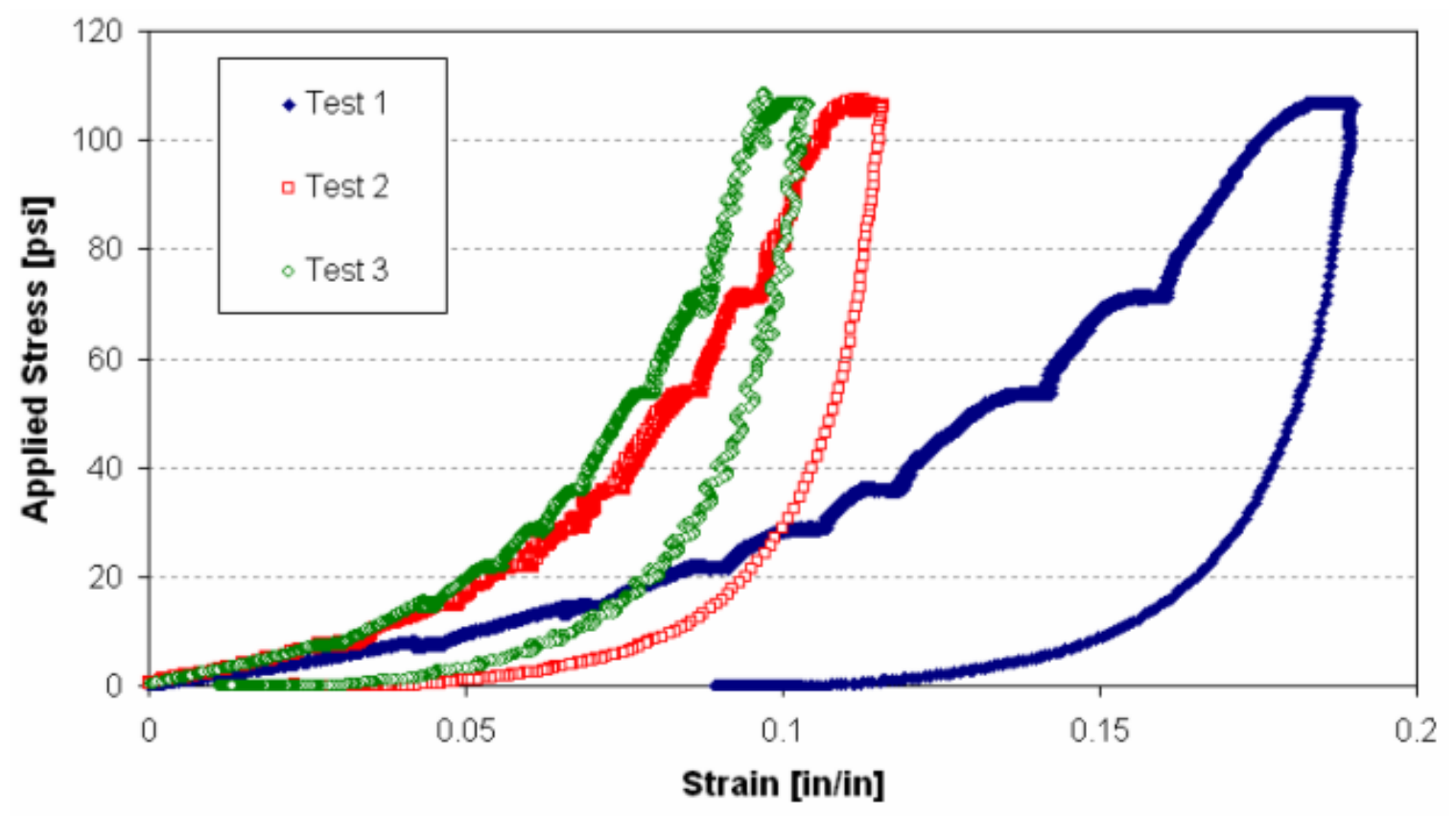

(a)

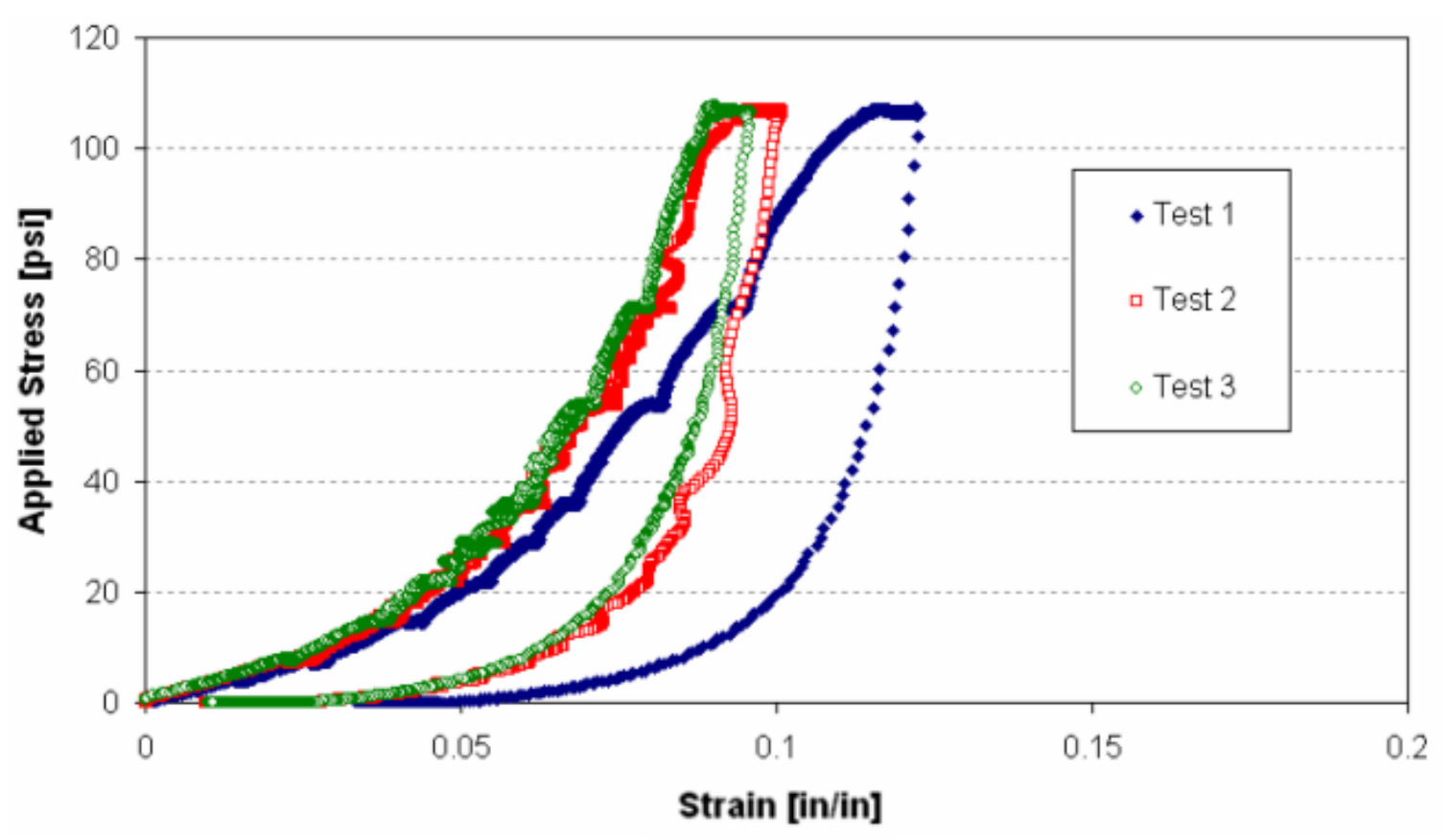

(b)

Figure 3.15: Stress-Strain Data Collected from the Sustained Loading Tests Conducted on Specimen (a) C2WC04 \#2, and (b) C2WC057 \#1 


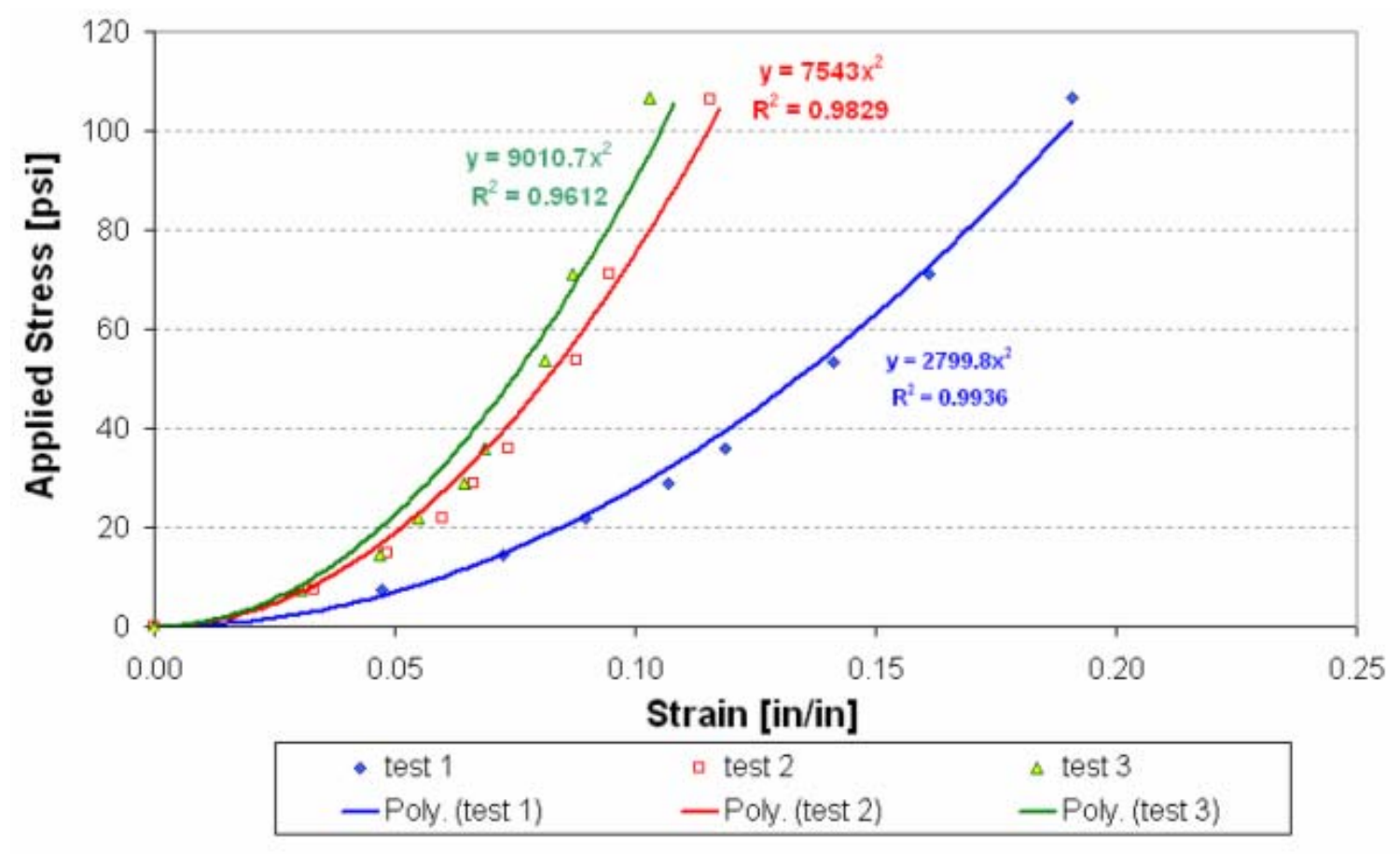

(a)

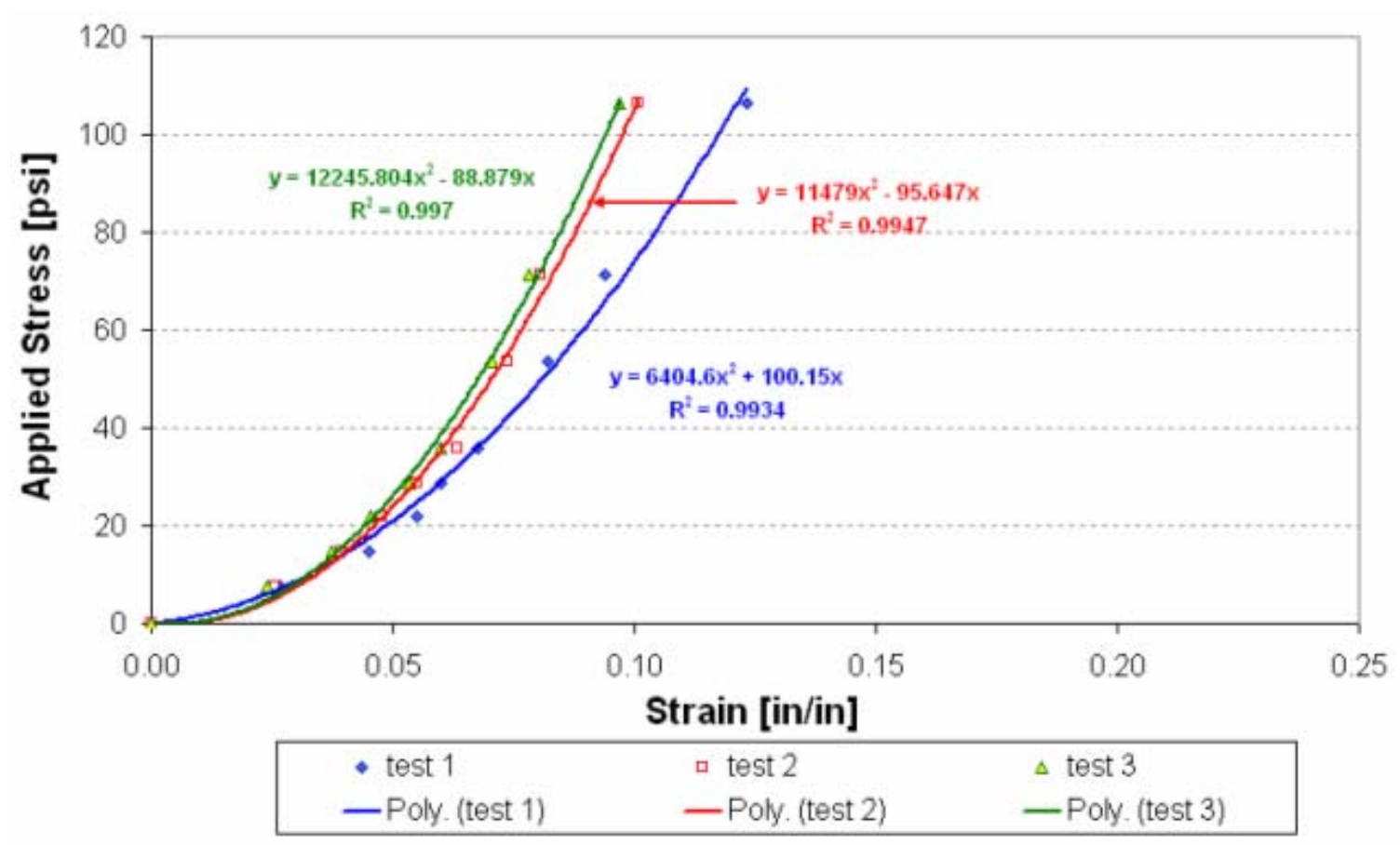

(b)

Figure 3.16: Trendlines Derived from the Sustained Loading Tests Conducted on Specimen (a) C2WC04 \#2, and (b) C2WC057 \#1 


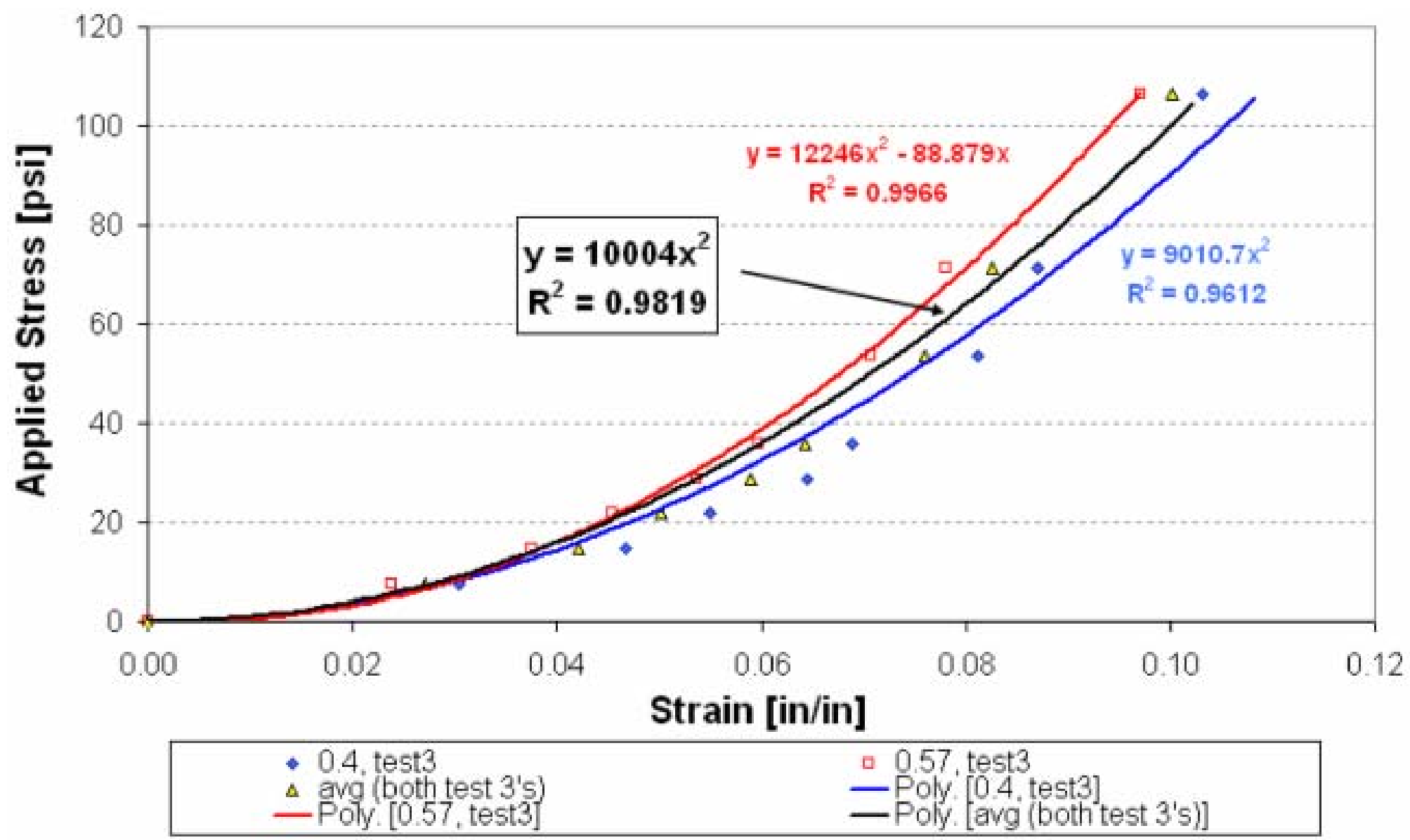

Figure 3.17: Comparison of Trendlines Derived from the Final Sustained Loading Tests Conducted on Specimens C2WC04 \#2 and C2WC057 \#1 




Figure 3.18: Comparison of Characteristic Stress-Strain Curves for Loads up to 100 psi

*** Load-Release Curves were developed at much higher stress levels and may be inaccurate at such low stress values $* * *$ 


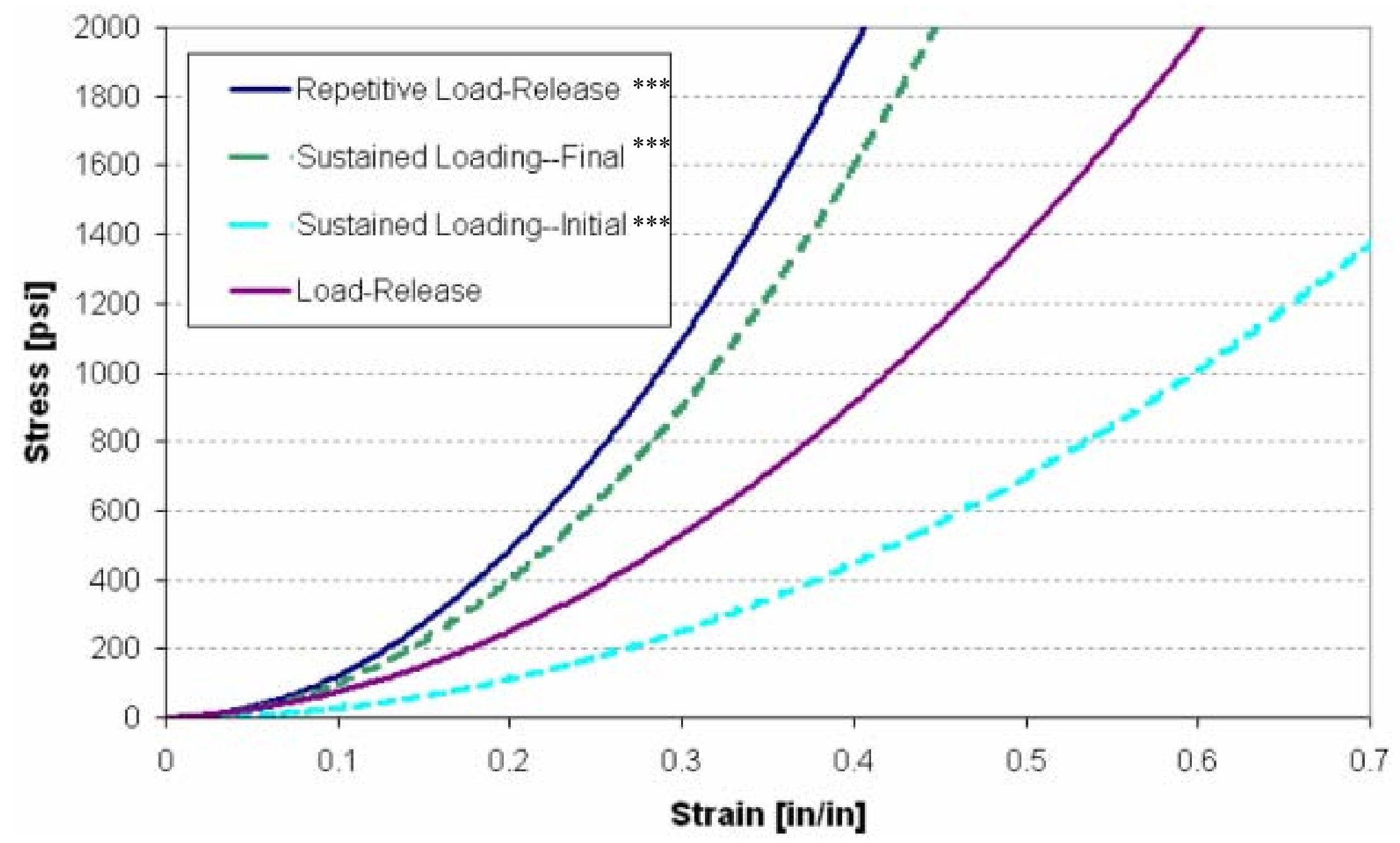

Figure 3.19: Comparison of Characteristic Stress-Strain Curves for Loads up to 2,000 psi

*** Repetitive Load-Release, and both Sustained Loading Curves were developed at much lower stress levels and may be inaccurate at stress values above $100 \mathrm{psi} * * *$ 


\section{CHAPTER 4 \\ CYCLIC FATIGUE TESTS}

\subsection{Overview}

In order to see if there would be any deterioration of Roadrain properties after it was exposed to numerous load cycles, a testing scheme based upon that of the dynamic complex modulus tests discussed in Chapter 2 was devised to compare the amount of plastic deformation at various points in time during the fatigue tests. From the tests of two specimens tested, various properties of Roadrain were established at predetermined numbers of cycles; among these were the elastic strain, accumulated plastic strain, and total strain for a fixed load range. Also, at the end of each test the rebound deflection for the sample was recorded during unloading.

\subsection{Test Setup and Procedure}

For the fatigue tests, it was decided to use the floor as a sub-grade in order to eliminate uncertainties in measurements that may come from using soil sub-grades. As far as Roadrain performance is concerned, this setup is believed to represent the "worstcase" scenario when considering wear and tear on the Roadrain, so acceptable performance of the Roadrain in this case would also suggest acceptable performance characteristics when placed on the softer, more forgiving soil sub-grades that would be used in the field.

\subsubsection{Equipment and Instrumentation}

The setup for the experiments is shown in Figures 4.1 and 4.2. A 110-kip MTS actuator was used to apply the load, which was distributed to the Roadrain layer via a pre-cast 2'x2'x11" concrete block. Loadings were controlled using an MTS 407 control system. The deflection of the Roadrain was ascertained using two LVDTs, which were placed on opposite sides of the block. Deflection data was read and recorded with a personal computer via a Smartstrain 5000 data acquisition system. 


\subsubsection{Procedures}

The applied cyclic compressive loading was sinusoidal in nature with magnitudes ranging from 0 to $20,000 \mathrm{lbs}$, or stress levels from approximately 0 to $35 \mathrm{psi}$. The rate of loading was $3 \mathrm{~Hz}$, but varied at the times when readings were taken. The durations of recording of the deflections also varied, but measurements were taken for at least 10 loading cycles in all cases. Table 4.1 shows the rates at which readings were taken for each specimen with the corresponding cumulative number of loading cycles undergone before the measurements were taken. For example, it can be seen from the table that for Specimen 1 at 400,000 cycles, readings were taken at three different rates of loading; this was done to observe the effects from the loading rate on the deflection curves.

In addition to the data collected at the aforementioned times during the fatigue tests, upon completion of 1,000,000 cycles, deflections were recorded during the unloading of each test specimen. Due to the nature of the control system for the actuator, the amplitude of the cyclic loading was first reduced to zero, leaving a static load of $10,000 \mathrm{lbs}$ still applied to the specimen. At that point, the load could then be fully removed from 10,000 $\mathrm{lbs}$ to $0 \mathrm{lbs}$ (see Figure 4.3 for an example of the deflections occurring as a result of the unloading process). Deflection data was taken from each sample throughout this process until some time after the load had been completely removed to measure the unloading stiffness.

\subsection{Experimental Results}

The average of the deflections recorded from the LVDTs at each time gave sinusoidal curves that mimicked the loading cycle. Figure 4.4 shows a sample curve from one such data set. From these sine curves, the maximum and minimum deflections for each data set made it possible to determine the accumulated plastic strain, the elastic strain, and the resilient modulus of the Roadrain sample for each data set. The progressions of these characteristics throughout both tests are shown in the charts of Figures 4.5, 4.6, and 4.7, respectively. From these figures, it can be seen that as the number of cycles increases there is a general increase in the amounts of both accumulated

plastic strain and resilient modulus, with a reduction in elastic strain. Some such values are tabulated in Tables 4.2(a) and (b), which show the percent changes over selected time 
intervals for accumulated plastic strains and for resilient moduli. From these tables, it can be seen that there is an average increase in accumulated plastic strain of almost $50 \%$ by 1,000,000 cycles, most of which occurs before 100,000 cycles. Also there is an average increase in resilient modulus of more than $100 \%$, only a small portion of which occurs after 500,000 cycles. These relationships could be useful in cases where it is desirable to model the long-term highway behavior after it has been subjected to many cycles of traffic loading.

The maximum values for the accumulated plastic deformation of the Roadrain samples at the conclusion of the application of loading for the tests ranged from $0.072 \mathrm{in}$. for the $0.1 \mathrm{~Hz}$ loading of Sample \#2 to $0.088 \mathrm{in}$. for the $1 \mathrm{~Hz}$ loading of Sample \#1. From this, it is seen that Roadrain can be expected to undergo an overall reduction of thickness of approximately $14-17 \%$ in relation to its initial thickness of $0.5 \mathrm{in}$. if subjected to loads of this magnitude throughout its serviceable life.

The values obtained from these tests for the initial resilient moduli are reasonable when considering the results of the uniaxial vertical compression tests discussed in Chapter 3. The nature of cyclic fatigue tests is most like that of the repetitive loadrelease tests discussed in Section 3.3.4 in the sense that they both have short durations of loading, so the load-deflection data used to calculate the stress-strain relationship is not significantly reflective of the creep behavior present in that of the sustained loading tests. The stress-strain relationship obtained from the repetitive load-release compression tests, as discussed in Section 3.4.4 $\left(\sigma=12,156 \varepsilon^{2}\right)$, would give a secant modulus of approximately 650psi when considering the stress range used for the cyclic fatigue tests, 0 to 35 psi. This is higher than the resilient modulus calculated for the onset of testing of Sample \#2 when loaded at $0.1 \mathrm{~Hz}, 570 \mathrm{psi}$; this load rate, the lowest of the three frequencies used for the cyclic fatigue tests, is the closest to the slower loading of the vertical compression test specimens. The fact that the Roadrain used for the vertical compression tests was bonded to concrete on one side may explain the counter-intuitively higher stiffness than that which was obtained with a higher load rate during the cyclic fatigue tests; the penetration of the cement paste into the fabric on the bonded side would most likely account for this difference. 
The data from the unloading of each specimen was also recorded; Figure 4.3 shows the curve obtained from Specimen 2. While there was no load-time history acquired to create a load-displacement curve, the known starting and ending loads and the corresponding displacements made it possible to determine the unloading stiffness and unloading modulus for each specimen; these are shown in Table 4.3. 
Table 4.1: Rate(s) of Loading during Readings of Deflection Data and Number of Cycles Completed before the Reading for Two Fatigue Tests

\begin{tabular}{|c|c|c|}
\hline $\begin{array}{c}\text { Number of } \\
\text { Cycles+ }\end{array}$ & $\begin{array}{c}\text { Specimen 1 } \\
\mathbf{( H z )}\end{array}$ & $\begin{array}{c}\text { Specimen 2 } \\
\mathbf{( H z )}\end{array}$ \\
\hline \hline $\mathbf{0}$ & 1.0 & $0.1,1.0$ \\
\hline $\mathbf{5 , 0 0 0}$ & 1.0 & $0.1,1.0$ \\
\hline $\mathbf{1 0 , 0 0 0}$ & 1.0 & $0.1,0.25,1.0$ \\
\hline $\mathbf{2 0 , 0 0 0}$ & 1.0 & $0.1,0.25,1.0$ \\
\hline $\mathbf{5 0 , 0 0 0}$ & 1.0 & $0.1,0.25,1.0$ \\
\hline $\mathbf{1 0 0 , 0 0 0}$ & 1.0 & $0.1,0.25,1.0$ \\
\hline $\mathbf{2 0 0 , 0 0 0}$ & 1.0 & $0.1,0.25,1.0$ \\
\hline $\mathbf{3 0 0 , 0 0 0}$ & 1.0 & $0.1,0.25,1.0$ \\
\hline $\mathbf{4 0 0 , 0 0 0}$ & $0.1,0.25,1.0$ & $0.1,0.25,1.0$ \\
\hline $\mathbf{5 0 0 , 0 0 0}$ & $0.1,0.25,1.0$ & $0.1,0.25,1.0$ \\
\hline $\mathbf{6 0 0 , 0 0 0}$ & $0.1,0.25,1.0$ & $0.1,0.25,1.0$ \\
\hline $\mathbf{7 0 0 , 0 0 0}$ & $0.1,0.25,1.0$ & $0.1,0.25,1.0$ \\
\hline $\mathbf{8 0 0 , 0 0 0}$ & $0.1,0.25,1.0$ & $0.1,0.25,1.0$ \\
\hline $\mathbf{9 0 0 , 0 0 0}$ & $0.1,0.25,1.0$ & $0.1,0.25,1.0$ \\
\hline $\mathbf{1 , 0 0 0 , 0 0 0}$ & $0.1,0.25,1.0$ & $0.1,0.25,1.0$ \\
\hline & &
\end{tabular}

+Number of loading cycles completed before readings of deflection were taken. Rate of loading was $3 \mathrm{~Hz}$ between the indicated numbers of cycles. 
Table 4.2: Changes of Material Properties of Roadrain at Selected Points as Observed During the Cyclic Fatigue Tests (a) Accumulated Plastic Strain, and (b) Resilient Modulus

\begin{tabular}{|c|c|c|c|c|c|c|}
\hline \multirow{2}{*}{$\begin{array}{c}\text { Cylinder Number / } \\
\text { Loading Rate }\end{array}$} & \multicolumn{6}{|c|}{ (a) \% Increase of Accumulated Plastic Strains at Indicated Nos. of Cycles } \\
\hline & $0 \mathrm{k} \rightarrow 200 \mathrm{k}$ & $0 \mathrm{k} \rightarrow 500 \mathrm{k}$ & $0 \mathrm{k} \rightarrow 1000 \mathrm{k}$ & $100 \mathrm{k} \rightarrow 1000 \mathrm{k}$ & $200 \mathrm{k} \rightarrow 1000 \mathrm{k}$ & $500 \mathrm{k} \rightarrow 1000 \mathrm{k}$ \\
\hline $1 / 0.1 \mathrm{~Hz}$ & $\begin{array}{c}-- \\
\end{array}$ & $\overline{---}$ & $\overline{-1--}$ & $\begin{array}{c}-- \\
\end{array}$ & $\begin{array}{c}-- \\
\end{array}$ & $9.1 \%$ \\
\hline $1 / 0.5 \mathrm{~Hz}$ & --- & --- & --- & --- & --- & $8.2 \%$ \\
\hline $1 / 1.0 \mathrm{~Hz}$ & $37.7 \%$ & $46.2 \%$ & $56.8 \%$ & $15.6 \%$ & $13.9 \%$ & $7.3 \%$ \\
\hline $2 / 0.1 \mathrm{~Hz}$ & $48.2 \%$ & $49.7 \%$ & $57.9 \%$ & $11.4 \%$ & $6.5 \%$ & $5.5 \%$ \\
\hline $2 / 0.5 \mathrm{~Hz}$ & --- & --- & --- & -- & $6.0 \%$ & $4.5 \%$ \\
\hline $2 / 1.0 \mathrm{~Hz}$ & $18.6 \%$ & $19.6 \%$ & $23.9 \%$ & $7.4 \%$ & $4.5 \%$ & $3.5 \%$ \\
\hline AVERAGE & $34.9 \%$ & $38.5 \%$ & $46.2 \%$ & $11.5 \%$ & $7.7 \%$ & $6.4 \%$ \\
\hline
\end{tabular}

\begin{tabular}{|c|c|c|c|c|c|c|}
\hline \multirow{2}{*}{$\begin{array}{l}\text { Cylinder Number / } \\
\text { Loading Rate }\end{array}$} & \multicolumn{6}{|c|}{ (b) \% Increase of Resilient Moduli at Indicated Nos. of Cycles } \\
\hline & $0 \mathrm{k} \rightarrow 200 \mathrm{k}$ & $0 \mathrm{k} \rightarrow 500 \mathrm{k}$ & $0 \mathrm{k} \rightarrow 1000 \mathrm{k}$ & $100 \mathrm{k} \rightarrow 1000 \mathrm{k}$ & $200 \mathrm{k} \rightarrow 1000 \mathrm{k}$ & $500 \mathrm{k} \rightarrow 1000 \mathrm{k}$ \\
\hline $1 / 0.1 \mathrm{~Hz}$ & --- & --- & --- & --- & --- & $6.5 \%$ \\
\hline $1 / 0.5 \mathrm{~Hz}$ & --- & --- & --- & --- & --- & $2.3 \%$ \\
\hline $1 / 1.0 \mathrm{~Hz}$ & $55.7 \%$ & $142.2 \%$ & $133.9 \%$ & $5.7 \%$ & $3.7 \%$ & $-3.4 \%$ \\
\hline $2 / 0.1 \mathrm{~Hz}$ & $88.9 \%$ & $96.2 \%$ & $125.0 \%$ & $31.3 \%$ & $19.1 \%$ & $14.7 \%$ \\
\hline $2 / 0.5 \mathrm{~Hz}$ & -- & -- & -- & $24.1 \%$ & $18.7 \%$ & $8.3 \%$ \\
\hline $2 / 1.0 \mathrm{~Hz}$ & $54.5 \%$ & $59.7 \%$ & $78.4 \%$ & $21.0 \%$ & $15.4 \%$ & $11.7 \%$ \\
\hline AVERAGE & $66.4 \%$ & $99.3 \%$ & $112.4 \%$ & $20.5 \%$ & $14.2 \%$ & $6.7 \%$ \\
\hline
\end{tabular}


Table 4.3: Unloading Stiffnesses and Moduli as Observed at the Conclusion of Each Respective Fatigue Test

\begin{tabular}{|c|c|c|c|c|c|c|}
\hline \multirow{2}{*}{ SAMPLE } & \multicolumn{2}{|c|}{ APPLIED LOAD } & \multicolumn{2}{|c|}{ DEFORMATION } & \multirow{2}{*}{$\begin{array}{c}\text { UNLOADING } \\
\text { STIFFNESS [psi/in] }\end{array}$} & \multirow{2}{*}{$\begin{array}{l}\text { UNLOADING } \\
\text { MODULUS [psi] }\end{array}$} \\
\hline & INITIAL [psi] & FINAL [psi] & INITIAL [in] & FINAL [in] & & \\
\hline 1 & 17. & 0 & 0.0979 & 60 & 1454.0 & 727.0 \\
\hline 2 & 17.36 & 0 & 0.0822 & 0.0719 & 1685.5 & 842.8 \\
\hline
\end{tabular}




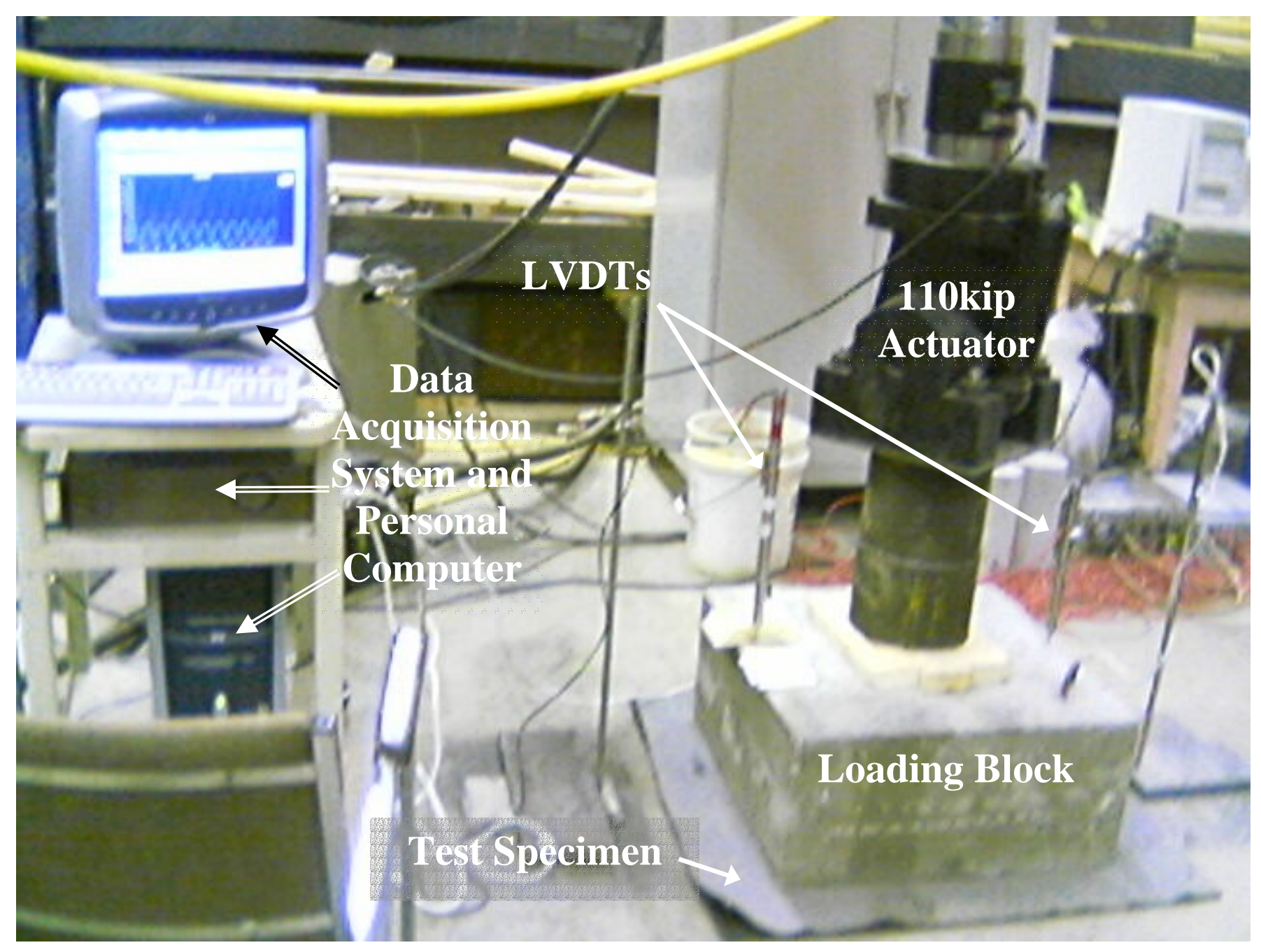

Figure 4.1: Overview of Cyclic Fatigue Testing Setup 


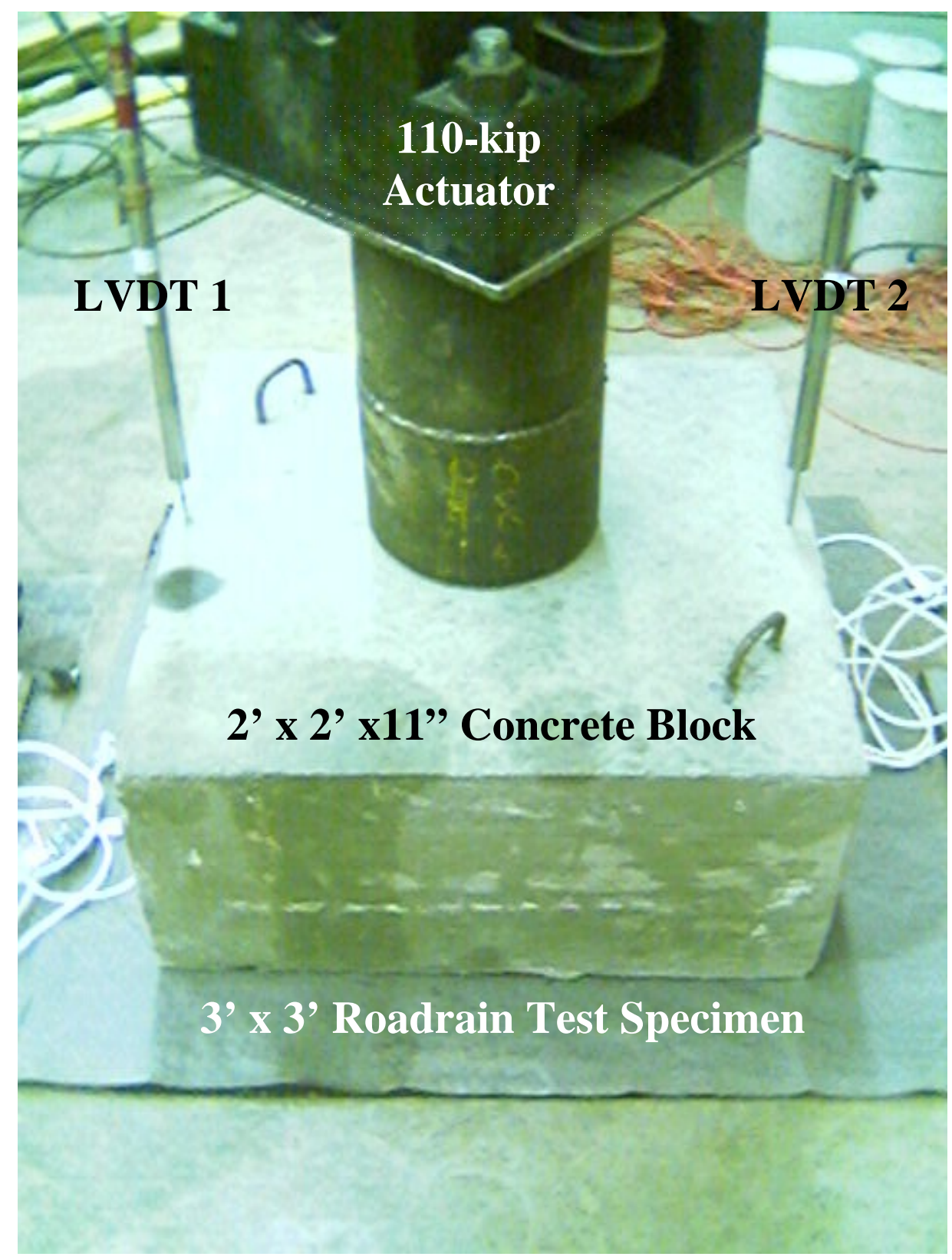

Figure 4.2: Cyclic Fatigue Test Specimen, Instrumentation, and Loading Block 


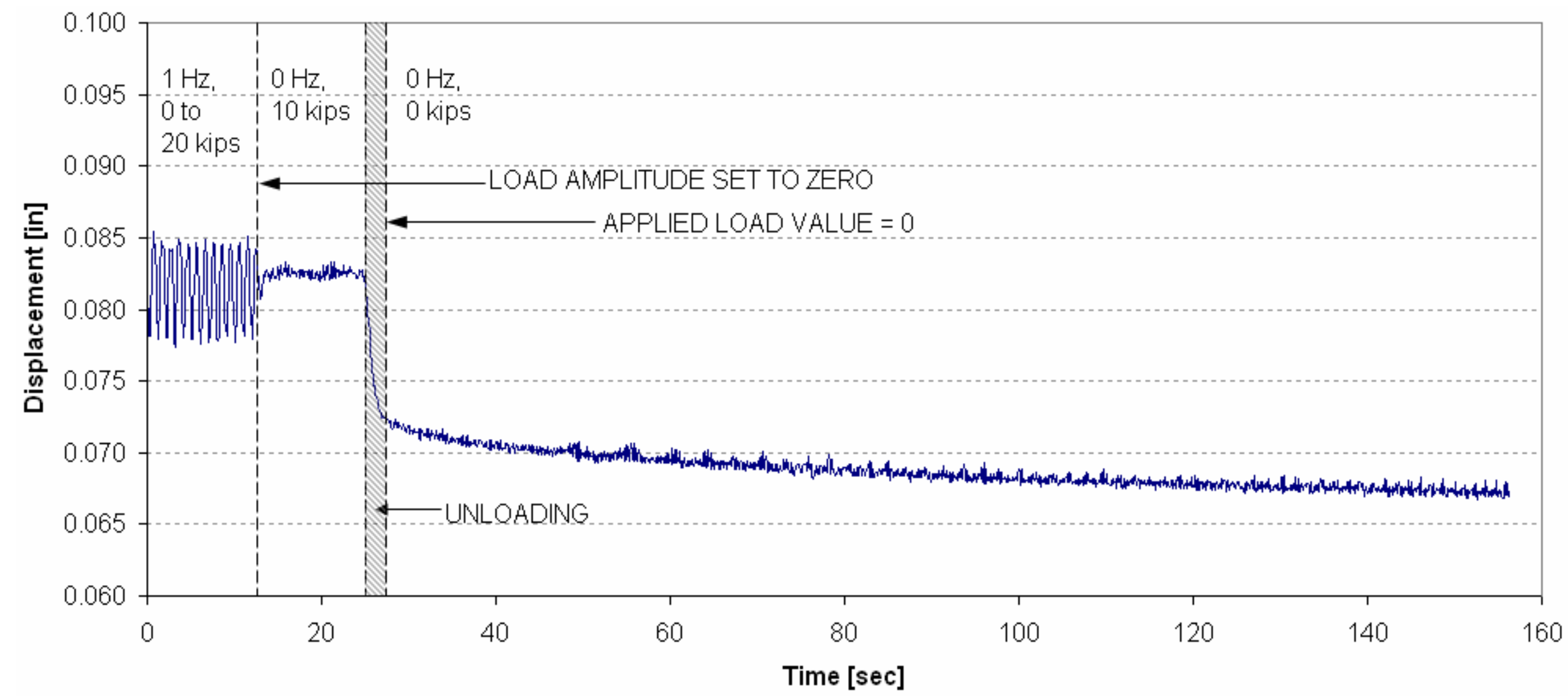

Figure 4.3: Unloading Data Obtained at the Conclusion of the Fatigue Test of Specimen 2 




Figure 4.4: Average Displacement vs. Time for Cyclic Fatigue Test of Specimen 2 after 20,000 Cycles Loading Range: 0 to 20,000 lbs; Loading Frequency: $0.25 \mathrm{~Hz}$

79 


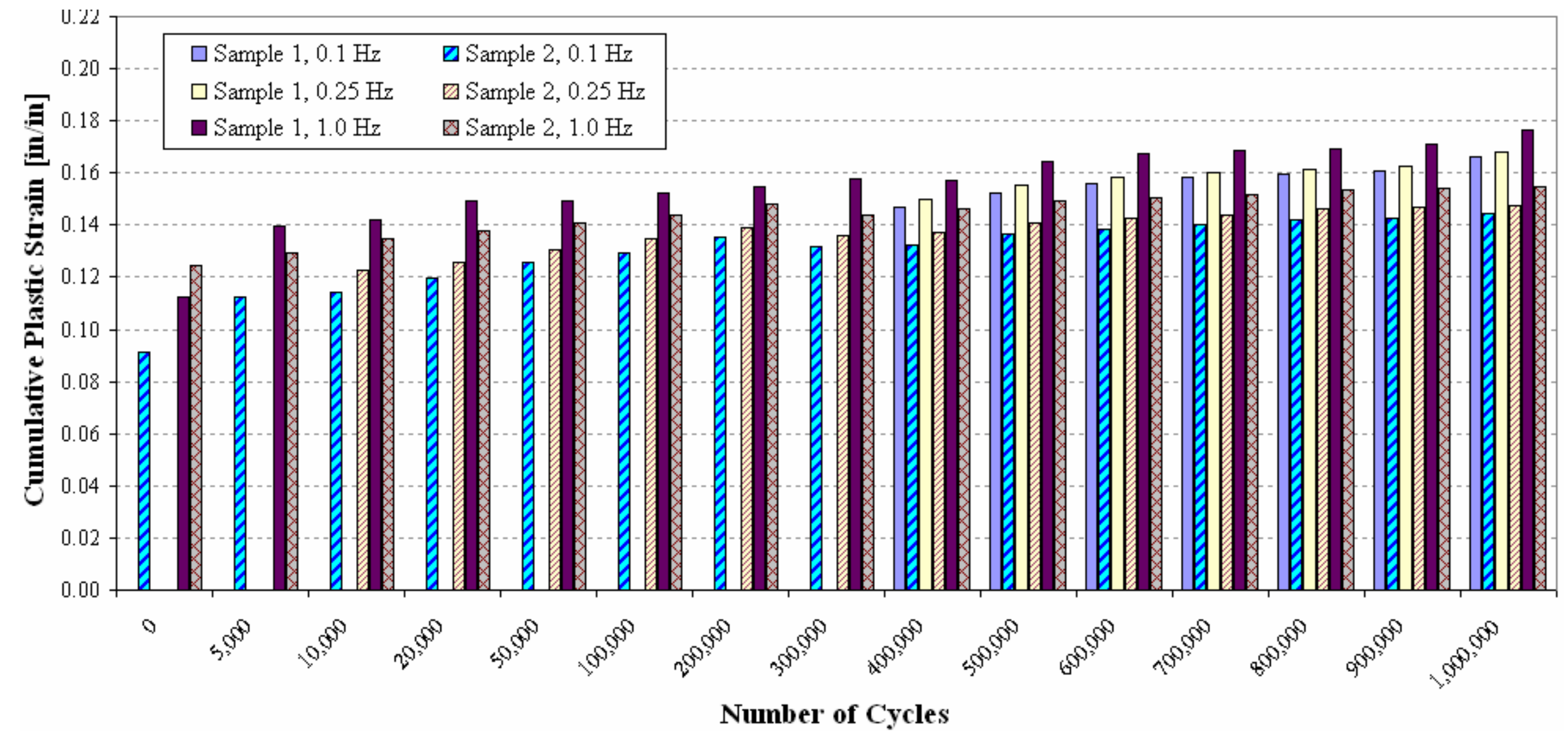

Figure 4.5: Cumulative Plastic Strain vs. Number of Cycles Both Samples Subjected to an Applied Cyclic Loading of 35 psi 


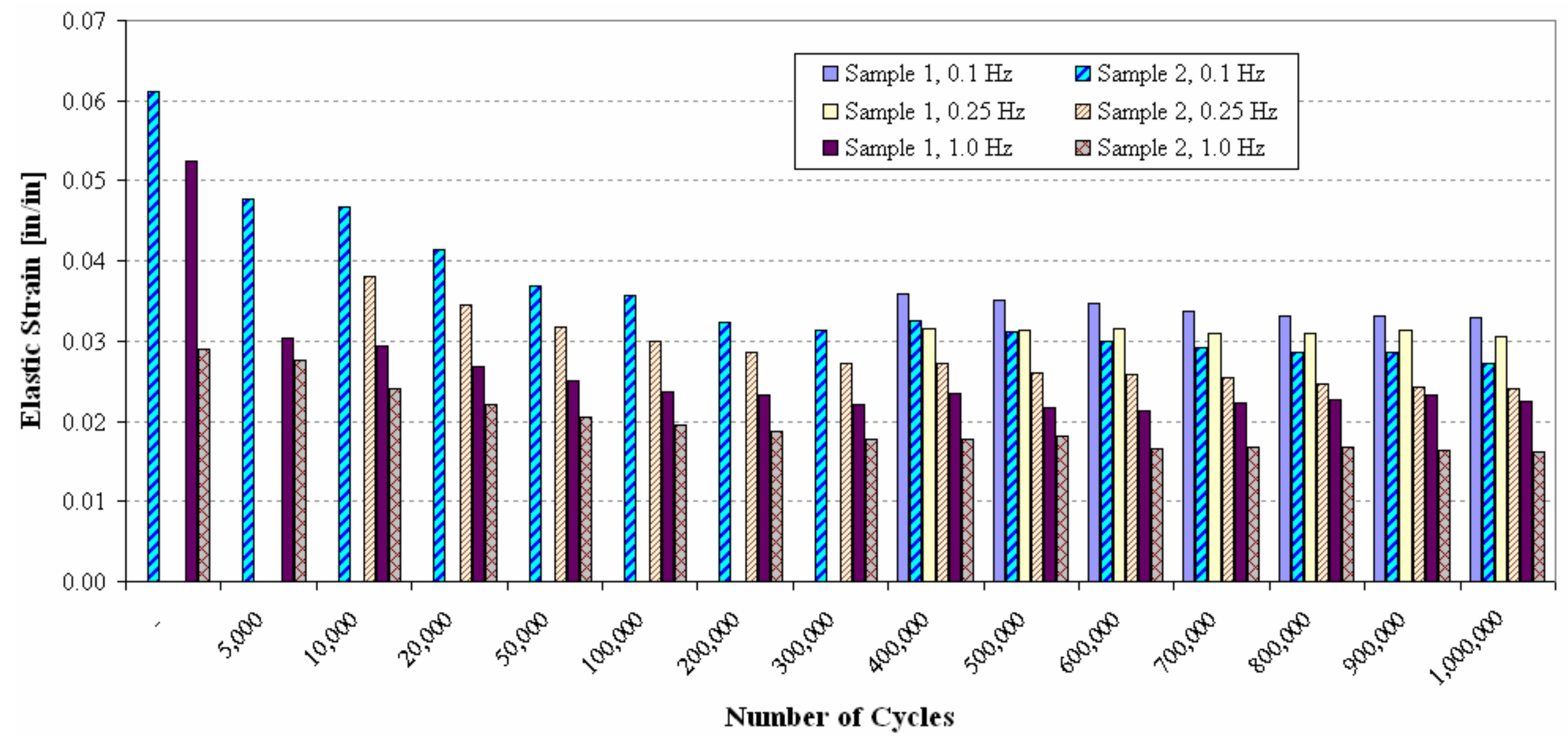

Figure 4.6: Elastic Strain vs. Number of Cycles

Both Samples Subjected to an Applied Cyclic Loading of 35 psi 


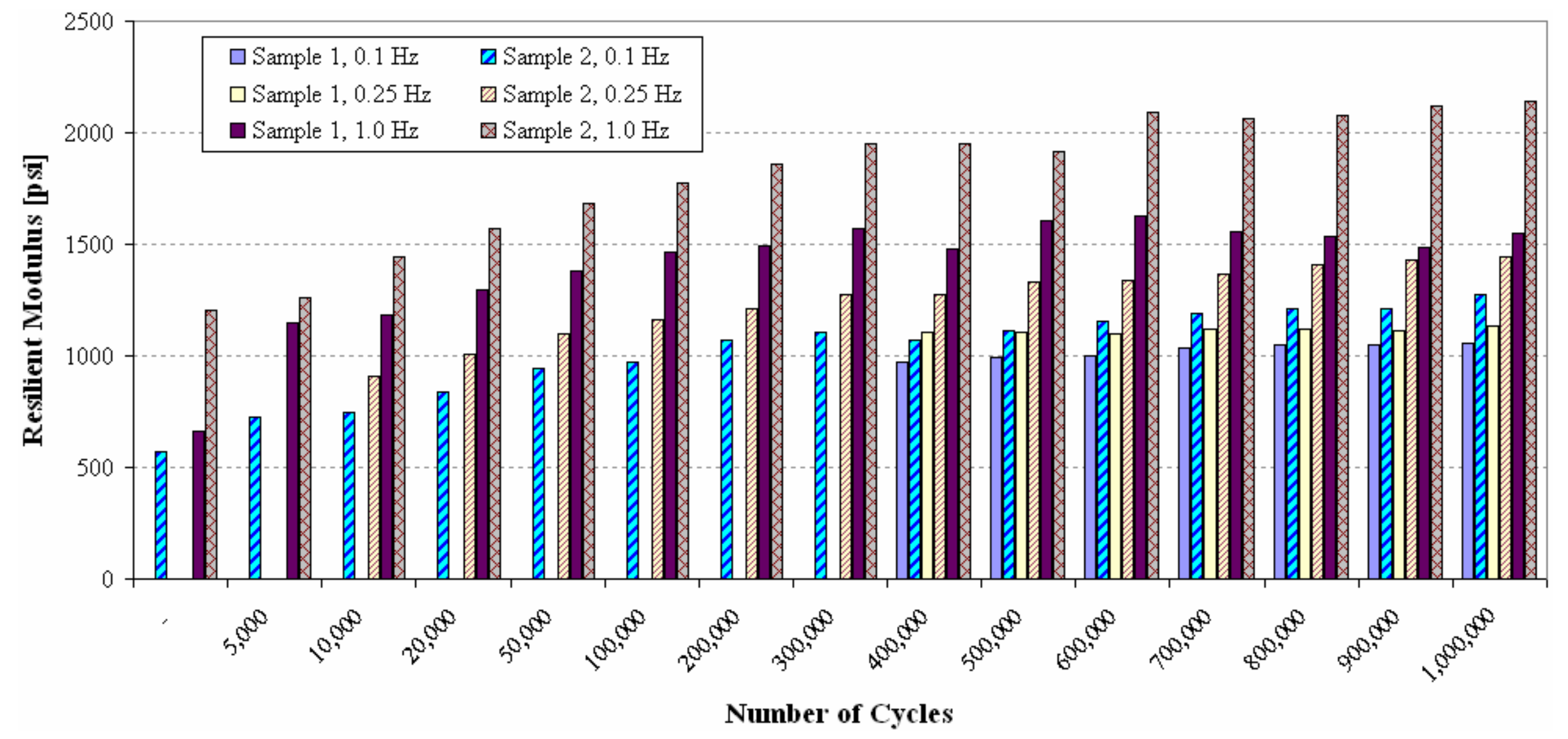

Figure 4.7: Roadrain Resilient Modulus vs. Number of Cycles Both Samples Subjected to an Applied Cyclic Loading of 35 psi 


\section{CHAPTER 5 \\ PLATE BEARING TESTS}

\subsection{Overview}

Two types of Plate Bearing Tests were run throughout the course of this study: Non-Repetitive Static Plate Bearing tests and Repetitive Static Plate Bearing tests. These were set up in a laboratory using two types of soils which were to simulate different types of in-situ slab support. The first type of subbase used was comprised of open-graded \#57 limestone, and the second used a soil which was excavated from a local construction site; for simplicity, these will be referred to throughout this and subsequent chapters as the limestone subbase and the A-2-4 soil subbase, respectively; the classification of the excavated soil as the AASHTO-defined A-2-4 soil will be discussed in the next section, Section 5.2. Both the Non-repetitive and the Repetitive tests were done using each soil type. In addition, some tests were done on the excavated soil for the purpose of classification and to obtain key mechanical properties. The test setups and procedures will be discussed in Section 5.3 of this chapter, and the results will be discussed in Section 5.4.

\subsection{Soil Classification}

In attempts of identifying some key properties of the unknown subbase used for the plate bearing tests, some laboratory experiments were conducted on the excavated soil. From the laboratory tests, the gradation, Atterburg limits, Angle of Friction, and cohesion of the soil were obtained. These tests were not repeated for the \#57 limestone aggregates; since the use of this general type of open-graded, granular aggregate is commonplace in highway and construction applications, so it has been studied in the past and its properties well documented.

\subsubsection{Soil Classification Tests}

Laboratory tests were conducted for classification of the excavated soil which was used as a subbase; these included a sieve analysis to determine gradation, and Liquid Limit and Plastic Limit tests to determine the soil's Atterburg Limits. Also, Direct Shear 
Tests were conducted to determine a value of cohesion, c, and an Angle of Friction, $\phi$, for the soil. All tests done for the purpose of soil classification are fairly basic tests in the world of soil mechanics, so their procedures and results will only be described only briefly throughout the remainder of this section.

\subsubsection{Initial Soil Preparation}

In preparation for all laboratory test for determining soil characteristics, all soil to be tested underwent a small period of preliminary preparation. First, a large amount of soil was oven dried for a period of 2 days in order to eliminate any moisture there within. Then, the oven dried soil was sent through a pulverizing machine in order to eliminate clumps that may be due to flocculation of clay particles. Once the moisture had been removed and the soil had been pulverized, it was portioned out for the various individual tests that will be described in the following sub-sections.

\subsubsection{Sieve Analysis Procedure}

The equipment and procedures used for the sieve analysis were in accordance with ASTM standard D 6913. The sieve sizes used for this test were designated, in order of decreasing opening diameter, as 4, 10, 20,40,60,100,140, 200, and PAN. These were first placed in a stack in the aforementioned order with the largest opening (\#4) sieve on top and the PAN on the bottom (see Figure 5.1). Approximately 500g of the prepared soil was placed in the top sieve and then a lid was secured, enclosing the soil inside. The stack was then placed in a mechanical sieve shaker for a period of approximately 5 minutes, and then removed and left to sit for another 5 minutes, thus allowing the dust to settle inside. At that point, the mass of soil contained in each sieve can be obtained for analysis.

\subsubsection{Plastic Limit Test Procedure}

The plastic limit is what we now call Atterburg's roll-out limit, and is representative of the moisture content at which clay can no longer be rolled into threads of approximately 1/8-inch diameter. ASTM D 4318 gives guidelines that were followed for determining the Plastic Limit. First, approximately $15 \mathrm{~g}$ of soil was mixed with 
enough water such that it could be rolled into a thread, and subsequently rolled to a diameter of about $1 / 8$ inch. The threads was then worked and kneaded, consequently removing water and somewhat drying it out. It was again rolled into a 1/8-inch diameter thread, and the process repeated until a point at which the soil crumbled under the forces necessary for rolling. At that point, the soil sample was placed in a closed container, the entire container with lid was weighed, the lid was removed and the container was placed in an oven for a period of 2 days to remove water from the soil, and then the container and lid were re-weighed such that the moisture content at the specimen at the point of crumbling could be determined. This was done for multiple samples and the results averaged.

\subsubsection{Liquid Limit Test Procedure}

The testing procedure for determining Atterburg's lower limit of fluidity, or what we now call the liquid limit, of a soil is described in ASTM D 4318. The equipment used for this test included a Soiltest liquid limit device and a grooving tool, as shown in Figure 5.2 , as well as some other standard soil laboratory equipment such as a spatula, scale, and small tin containers.

For this test, about $100 \mathrm{~g}$ of the dried, pulverized soil was first thoroughly mixed with water until it was at a point near the approximated liquid limit. At that point, a portion was placed in the dish on the liquid limit device and smoothed with a spatula to a thickness of approximately $1 \mathrm{~cm}$ at the deepest point. Then the grooving tool was used to form a groove from front to back down the center of the sample, as indicated by the dashed line in Figure 5.2. The crank on the liquid limit device was then turned at a rate such that the dish would raise and fall approximately twice every second. The crank was turned and the number of drops counted until a point at which the groove that was cut into the sample had closed along a length of approximately $1 / 2 \mathrm{inch}$. This was done multiple times until the same number of blows was obtained twice in a row for any given moisture content. At that point, a sample of the soil was placed in a container and the moisture content corresponding to that number of blows was determined in the same fashion as that of the Plastic Limit tests. The entire test procedure was repeated for four 
different moisture contents such that four corresponding numbers of blows would be obtained.

\subsubsection{Direct Shear Test Procedure}

The direct shear test was done in accordance with ASTM D 3080 using the direct shear setup shown in Figure 5.3. It can be seen from this figure that this device contains the following components: (1) a shear box containing the soil sample to be tested, (2) a loading arm for applying a known normal force to the sample, (3) a motorized device for applying and measuring a horizontal force to the sample, and (4) a deflectometer to measure the horizontal displacement of the sample at any time. The shear box is designed to allow horizontal movement of only a portion of the soil sample so to invoke a failure plane.

First, a sample of the dried, pulverized soil was placed into the shear box, and both were assembled into the appropriate location of the loading device. At that point, the vertical load was applied by placing the desired amount of weight onto the lever arm hanger. Since the soil was oven-dried and determined to be composed primarily of sand in the sieve analysis, consolidation of the sample under the normal force was virtually immediate. At that point, the horizontal force was applied at a steady rate (approximately $0.1 \mathrm{in} / \mathrm{min}$ ) while a computer read and recorded the horizontal force and the horizontal displacement of the soil at regular time intervals. This test was done two times each using the following normal loads: $5 \mathrm{~kg}, 10 \mathrm{~kg}, 15 \mathrm{~kg}$, and $20 \mathrm{~kg}$.

\subsubsection{Soil Classification Results}

From the sieve analysis, it was seen that the majority of particles (approximately $85 \%$ ) ranged in size from $0.1 \mathrm{~mm}$ to $1.0 \mathrm{~mm}$, indicating a high sand content for the soil; Figure 5.4 shows the gradation curve obtained from the sieve analysis. From this figure, the excavated soil was determined to be fairly well-graded, with an effective grain size, $\mathrm{D}_{10}$, of $0.073 \mathrm{~mm}$, a Uniformity Coefficient, $\mathrm{C}_{\mathrm{u}}$, of 5.5, and a Coefficient of Gradation, $\mathrm{C}_{\mathrm{c}}$, of 1.2. The definitions of these terms are as follows:

$$
C_{u}=\frac{D_{60}}{D_{10}} \quad \text { and } \quad C_{c}=\frac{D_{30}{ }^{2}}{D_{10} \times D_{60}},
$$


where $D_{10}, D_{30}$, and $D_{60}=$ Diameter of particle corresponding to 10,30 , and $60 \%$ finer, respectively.

Also, the Atterburg Limits were found via the Liquid Limit and Plastic Limit tests. The Plastic Limit, PL, of the soil was determined to be $18.0 \%$. A flow curve was developed from the Liquid Limit test data (see Figure 5.5), which gives a moisture content of $24.1 \%$ at the Liquid Limit, LL, for the soil, or in other words the moisture content corresponding to 25 blows. The determination of these factors allowed the Plasticity Index, PI, or the difference between LL and PL, to be calculated as $6.1 \%$.

In addition to the grading information and the Atterburg Limits of the soil, the Direct Shear Tests were necessary to classify the soil. For data reduction, the instantaneous contact area of the failure plane first needed to be calculated using the following relationship:

$$
A=A_{O}-w \cdot \Delta
$$

In this equation, $A$ represents the instantaneous area, $A_{0}$ is the initial area, w is the width of the specimen, and $\Delta$ is the gage reading of displacement at that time. Next, shear stress-shear strain curves with varying normal loads were constructed for each trial, as shown in Figure 5.6. These were used to obtain the shear stress which corresponds to $10 \%$ strain for each normal load. Finally, plotting the average of the two shear stresses at $10 \%$ strain versus the corresponding normal load for the test (see Figure 5.7) made it possible to determine a value for cohesion, c, and an Angle of Friction for the soil, $\phi$; the cohesion corresponds to the y-intercept of the straight line best-fit curve, and the angle of friction corresponds to the angle, in degrees, that that line makes when intersecting the $\mathrm{x}$ axis, or in other words $\phi=\tan ^{-1}$ (SLOPE). These relationships give a cohesion of $c=0$, and an angle of friction of $\phi=31^{\circ}$.

Using all the above information, the soil could then be classified. Using the ASTM method, or Unified Soil Classification System (USCS) specifications, the soil was identified as type CL $\neg$ ML, or "Gravelly Silty Clay with Sand." Also, AASHTO soil classification guidelines categorize the soil as type A-2-4, or "Silty or Clayley Gravel and Sand" (Das, 1998). It can be seen from Figure 5.8 that these soil types do not generally correlate in terms of typical mechanical properties, so no immediate conclusions about 
material stiffness can be drawn from the results of the soil classification procedures. Approximations of the Poisson's ratio can be made based upon the type of soil, though, by using Table 5.1; it can be seen from this table that for a silty and sandy soil such as the one tested that the Poisson's ratio would most likely fall between the values of 0.25 to 0.35 , depending upon the proportion of sand present.

\subsection{Plate Bearing Test Setup and Procedures}

The setup for the laboratory plate bearing tests was based upon the guidelines specified in ASTM D1195 and ASTM D1196 for the Repetitive and Non-repetitive Static Plate Testing of Soils, respectively. These guidelines are written with the intention of being used for the evaluation of thick in-situ pavement components and structures; these tests, however, adapted the guidelines for use with a laboratory setup including a thin subbase layer with or without a Roadrain fabric layer. All tests included a compacted, 6inch-deep subbase layer comprised either of \#57 Limestone aggregate or of the A-2-4 soil discussed in the previous section. The subbase was placed directly onto a rigid concrete floor, and enclosed on all sides by 1' x 1' x 10' concrete beams arranged in a rectangular fashion surrounding the loading frame that was used. For the tests in which a Roadrain layer was used, a 4' x 4' section was placed directly on top of the subbase. Figure 5.9 shows an overall view of the \#57 Limestone aggregate subbase layer and concrete beams with the Roadrain and instrumentation in place; the same equipment was used for all

plate bearing tests, with the only exceptions being the subbase type and the presence of Roadrain.

All tests pertaining to the \#57 Limestone aggregate subbase were completed (in the order shown in Table 5.1) before the tests involving the A-2-4 soil subbase were done (see Table 5.2). However, throughout the remainder of this and subsequent chapters the tests on both subbases pertaining to the Non-Repetitive Plate Bearing test procedure will be grouped together, as will all tests pertaining to the Repetitive Plate Bearing test procedure.

Figure 5.10 and Figure 5.11 show flow charts for the Non-Repetitive testing of the \#57 Limestone and A-2-4 soil subbases, respectively. It can be seen from these figures that after the test was run with the sheet of Roadrain, the subbase was disturbed 
and then re-compacted before being tested with another sheet of Roadrain; this was done as a check for the overall repeatability of the tests for each given subbase material. Also, Figures 5.12 and 5.13 show the flow charts for the Repetitive testing of the \#57 Limestone and A-2-4 soil subbases, respectively; the subbases were not disturbed or recompacted at any point throughout these tests. It should be noted for Figures 5.10 through 5.13, all boxes for tests marked "WITH Roadrain" indicate the implementation of a new, untested piece of Roadrain added to the designated state of the subbase, as the Roadrain layer was replaced for each set of tests.

\subsubsection{Equipment and Instrumentation}

As can be seen from Figure 5.9, a large loading frame and hydraulic jack were used to apply the forces necessary to conduct the plate bearing tests. The jack had a maximum capacity of 100 kips, and the jacking force was applied manually by a handoperated hydraulic pump. The jacking force was monitored by a $110 \mathrm{kip}$ load cell placed directly below the jack piston; the use of the load cell also allowed for the recording of vertical forces applied during testing.

The jacking force was transferred to the underlying layers via loading plates which were fabricated specifically for these tests according to the dimensions detailed in both ASTM D 1195 and ASTM D 1196. A set of five plates was used with diameters increasing in 6-inch increments, ranging from 6 to 30 inches. All plates were 1 in. thick and were composed of A36 steel. For testing, they were stacked in a pyramidal fashion, in order of decreasing diameter from bottom to top, as can be seen in Figures 5.14(a) and 5.14(b).

In addition to the load cell which was used, other forms of instrumentation were used to take measurements throughout the tests; in particular, two LVDTs and two dial gages were used to take vertical displacement measurements. These were arranged such that the two LVDTs would be offset $180^{\circ}$ from each other around the circumference of the plates, as would the dial gages. All displacement gages were set to measure deflections at a distance of approximately lin. from the outside of the largest plate; this was done by attaching the gages to one of two rigid bars which spanned the width of the soil subbase and rested on the concrete beams. Figure 5.15 shows a schematic overhead 
view of the test plates and instrumentation, and Figure 5.16 shows a schematic side view of the instrumentation as placed on the contained subbase.

As with the Vertical Compression tests, the Strainsmart 5000 Data Acquisition System was used to obtain both the deflection and applied loading data. In addition, deflection readings were taken and recorded using the dial gages at predetermined load values; these values acted simply to verify the more precise deflection data acquired using the LVDTs.

\subsubsection{Subbase Preparation}

In order to promote consistency among subbase properties for each test of either given subbase, efforts were made to provide uniform depth and compaction at the onset of testing and at selected points in between tests. To ensure a constant depth of 6 inches at the onset of testing for each subbase, marks were first made on the inside of the concrete containment beams at a height of 6 inches from the floor before any subbase materials were present. Then, the testing area was filled with the subbase material to a point slightly higher than the aforementioned marks and roughly leveled-off. At that

point, several passes of a vibrating plate tamper were used to compact the subbase while a screed across the soil ensured regular compaction and a level surface (see Figure 5.17). At certain points during compaction it was necessary to add or remove some soil in localized areas, so those particular sections were re-tamped with the plate tamper.

While the geometry of the testing area may have not made it possible to mechanically tamp some areas using the plate tamper, such as the corners around the loading frame, these areas were tamped as well as possible using hand tools. The areas in question are believed to be well outside of the zone of influence for such a shallow foundation, though, so this slight non-uniformity in compaction should not have influenced the overall behavior of the system.

\subsubsection{Non-Repetitive Static Plate Test Procedure}

As mentioned in a previous section, all laboratory Non-Repetitive Static Plate tests were devised based upon ASTM D1196 specifications for the in-situ evaluation of highway subgrade soils. The loading scenarios used were similar to those of the 
Sustained loading Vertical Compression tests in that incrementally larger loads were held at a constant value until the rate of deflection had declined significantly. At first a small load was applied and held constant until the rate of deflection became very small, and then the load was increased to a larger value. As suggested in ASTM D 1196, six loads (and corresponding deflections) were used to make the Load-Deflection relationships desired from this test. After the highest load had been applied and the resulting subbase deformation halted, the entire load was released and the eventual resulting rebound deflection was recorded.

The load scenarios used for all non-repetitive tests can be seen in the first rows of Tables 5.2 and 5.3. The same loads were used for both subbase types so the resulting load-deflection curves could be easily compared to one another. The numbers of times each loading pattern was applied for each non-repetitive test can be found on these tables in brackets after each respective loading scenario.

\subsubsection{Repetitive Static Plate Test Procedure}

The Repetitive Static Plate tests were done in consideration of ASTM D1195 specifications. For each test designation, the pre-determined load value was applied, held until the rate of deflection decreased substantially, was released until the rebound deflection stabilized, and then was reapplied and released in the same fashion multiple times; the process was similar to that of the Repetitive Load-Release tests described in Chapter 3 for the Vertical Compression tests. As mentioned before, the subbase soil was undisturbed during the times between tests.

The load values used for each repetitive test along with the order of testing for each subbase type and the number or repetitions of each test are given in the latter portions of Tables 5.2 and 5.3. Unlike for the non-repetitive tests, it can be seen that not only did variations in magnitude exist between similar tests for the two different subbase types (i.e. small load test without Roadrain for \#57 Limestone subbase vs. small load test without Roadrain for A-2-4 soil subbase), but load magnitude discrepancies also existed between similar loads for the small and medium load tests using the limestone subbase (i.e. small load test without Roadrain vs. small load test with Roadrain for \#57 Limestone subbase). This is because for the tests with the $\# 57$ limestone, the deflection-based 
guidelines of the ASTM guidelines for determining the appropriate loads were initially followed for these tests, but eventually it was decided that using similar load magnitudes, as was done for the A-2-4 soil subbase, would make for a better comparison between the support systems which did and did not incorporate a Roadrain layer.

\subsection{Experimental Results}

As was the case with the Vertical Compression Tests, numerous loaddisplacement relationships were established throughout the course of the Plate Bearing tests. The relationships given from the non-repetitive tests included load-displacement relationships for support systems with/without a Roadrain layer which were loaded in a fashion similar to that of the Sustained loading tests described in Chapter 3; this longterm loading scenario accounted for creep in measurements. Similarly, the relationships given from the repetitive tests included load-displacement relationships for support systems with/without a Roadrain layer which were loaded in a fashion similar to that of the Repetitive Load-Release tests described in Chapter 3; these shorter-term loading scenario did not account for creep in measurements, but focused primarily on the effects from multiple loadings on the support system. Also, the unloading curves resulting from all loading situations of all support systems were briefly investigated. The results from all Plate Bearing tests will be described and stress-displacement relationships will be given throughout this section; for simplicity, stress-strain relationships will not be given due to the different initial depths of supporting layers (6 in. for subbases without Roadrain vs. $6 \frac{1}{2}$ in. for subbases plus a Roadrain layer).

\subsubsection{Non-Repetitive Static Plate Bearing Test Data: Loading Curves}

For each Non-repetitive test, multiple curves were obtained which tracked the displacement histories of the supporting layers as a multi-step load was applied and then

released; a sample curve is shown in Figure 5.18, the curve representing the first loading of a compacted \#57 Limestone subbase plus a Roadrain layer. From these curves, stressdisplacement plots were made using an area of load application of $707 \mathrm{in}^{2}$, representing the area of contact for the largest plate. A plot containing all stress-displacement curves obtained from the Non-Repetitive Static Plate Bearing tests done on \#57 Limestone 
aggregate subbases can be seen in Figure 5.19, and those from tests done on A-2-4 soil subbases can be seen in Figure 5.20.

From Figures 5.19 and 5.20 it can be seen that all supporting systems generally underwent stiffening when comparing their first loading to subsequent trials, evident by the smaller deflections at the same loads, (the first and last trials of each supporting system are highlighted with thicker lines). This may be clearer when observing Figure 5.21 , which shows the deflection history of only one sample throughout multiple trials. This stiffening trend is consistent with the observations of Roadrain behavior as seen in Chapters 3 and 4, as well as with general soil behaviors when dealing with soil systems without Roadrain.

It can also be seen from both figures that the supporting systems which incorporate a re-compacted subbase as well as a Roadrain layer eventually behave very similar to those which incorporate Roadrain on top of a previously-tested subbase, even though the initial behaviors may be significantly different due to the different levels of subbase compaction. For this reason, the primary focus of all the Plate Bearing test analyses will be on the eventual, more repeatable stress-displacement relationships that occur after only a few trials. Therefore, using the final trial for each type of support system, characteristic curves were developed to describe the eventual stress-displacement relationship of each support system. The data points used to create these curves are the

points corresponding to the maximum deflections at the end of each sustained load. The resulting characteristic curves can be seen in Figures 5.22 and 5.23 for the tests using \#57 Limestone and A-2-4 soil subbases, respectively; these give equations for the eventual stress-displacement relationships of the limestone subbase, the average of the limestone subbases incorporating a Roadrain layer, the A-2-4 soil subbase, and the average of the A-2-4 soil subbases incorporating a Roadrain layer.

\subsubsection{Repetitive Static Plate Bearing Test Data: Loading Curves}

For each Repetitive Plate Bearing test that was run, the pavement support system tested was exposed to multiple cycles of loading and unloading. Figure 5.24 shows a sample load-deflection curve produced from the data that was taken continuously 
throughout the test. From this data, multiple load-displacement curves were obtained for each type of subbase tested with each load magnitude.

As was the case with the Repetitive Load-Release test data from the Vertical Compression testing described in Chapter 3, the plastic deformation present at the beginning of each load portion of the loading cycle was first removed to normalize the origin of the displacement for each trial. Figure 5.25 shows a comparison of (a) the original and (b) the normalized stress-displacement curves for the repetitive testing of an A-2-4 soil subbase below a Roadrain layer with an applied load of a 16 kips. It can be seen from this figure, particularly 5.25(b), that not only does the subbase/Roadrain system undergo stiffening as occurred in previous tests, but the support system also quickly approaches a point at which results become fairly repeatable with only small differences in displacements between successive load applications. This trend was present in all instances for the Repetitive Static Plate Bearing tests.

From the adjusted stress-displacement curves for all repetitive plate tests, characteristic curves were developed representing the eventual behavior of each supporting system. As was the case with the Non-Repetitive tests, the final trial for each support type was used as a basis of classification of this eventual behavior. Figures 5.26 and 5.27 show the characteristic curves obtained from the medium-load and large-load tests involving the \#57 Limestone aggregate and the A-2-4 soil subbases, respectively. Due to the much smaller range of stresses present in the small-load tests, these characteristic curves are all plotted together in Figure 5.28.

Figure 5.26 shows a good repeatability among the Repetitive Static Plate Bearing tests using the \#57 Limestone aggregate subbases. As can be seen from this figure, the applications of both the medium loads and large loads on the aggregate subbase alone invoke essentially the same stress-displacement relationship within the mutual stress range; this was the case for the support system which did incorporate a Roadrain layer, as well. The A-2-4 soil subbases, on the other hand, did not follow as closely to each other when tested without the Roadrain layer, as can be seen in Figure 5.27, but closer trends were present when the soil subbase was tested with Roadrain; the reasons for the discrepancies in the tests without Roadrain are not certain at this time. 


\subsubsection{Comparison of Loading Curves from Plate Bearing Tests}

Figures 5.29 and 5.30 show the stress-displacement plots of the various characteristic loading curves obtained from the Repetitive and Non-Repetitive Static Plate Bearing tests of the \#57 Limestone and the A-2-4 soil subbases, respectively. In these graphs, the curves from the Non-Repetitive Plate Bearing tests define the stressdisplacement of the support systems based on long-term loading which included creep, and the Repetitive tests define that based on shorter-term loadings which do not summon as drastic of a creep effect.

It can be seen from Figure 5.29 that in the tests involving a \#57 Limestone aggregate subbase, the Repetitive Plate Bearing tests produce stress-displacement curves similar to those of the Non-Repetitive tests. This is reasonable for the open-graded, granular subbase, since much of the compaction would be expected to occur almost instantaneously with very little creep effect. It is somewhat surprising, though, that no significant creep effect was discovered in the cases where a Roadrain layer was used, since this was seen in the Vertical Compression tests to experience time-dependent deformations. This creep effect, however, was seen to be small after multiple loadings and at small stress levels (refer to Figure 3.18), so the fact that no significant creep effect was seen could be attributed the normal range of experimental error, as well as to the nature of how the characteristic curves were developed (the characteristic curves are not necessarily along the path of experimental data at all points).

The curves of Figure 5.30, on the other hand, show that the A-2-4 soil does experience some degree of creep. This is more evident in the tests of the A-2-4 soil subbase with Roadrain than it is in the tests of the A-2-4 soil subbases alone. The tests of the soil subbase with the Roadrain layer behave as expected, with the Non-Repetitive tests producing more deflection at the same load due to the creep effect from the longterm loading. The results from the tests of the soil subbase alone do not have the expected trends that those tested with the Roadrain layer possessed though. The reasons for this could be the same as those discussed at the end of the preceding paragraph, but with greater effects than those of the \#57 Limestone subbase curves. It can still be seen from the tests of the soil subbase alone that if the trendline for the Non-Repetitive portion of testing is extended beyond the stress range in which it was tested, creep effects would 
be evident at higher stress ranges, even though the Repetitive test characteristic curves lack the consistency as those from the other support systems.

\subsubsection{Static Plate Bearing Test Data: Unloading Curves}

In addition to the loading curves, the unloading curves from all Repetitive and Non-Repetitive Static Plate Bearing tests were studied. These will not be described in great depth, though, since the unloading stress/rebound deflection relationships from these tests (1) at this point cannot give equations that characterize the overall rebound behavior of the support system, for the same reasons discussed in the results of Chapter 3, and (2) are dependent upon the subbase behavior as well, so additional analysis would need to be conducted in order to determine the separate stiffness contributions of the subbase and the Roadrain layer. For these reasons, only a sample of the data from the unloading of the Plate Bearing tests is shown in Figure 5.31; the rest will be documented in Appendix B, though. These curves generally had shapes which mimicked those of the Vertical Compression Tests, but with some inconsistencies; the increase in deviations from the typical patterns were most likely due to the sudden nature of the release of the load in the Plate Bearing tests, whereas the release was more gradual when controlled by the Baldwin loading machine used in the Vertical Compression tests.

\subsubsection{Summary of Results: Plate Bearing Tests}

From the Plate Bearing tests, stress-displacement relationships were established to characterize the behavior of various support systems after being exposed to numerous loadings. Characteristic stress-displacement curves were developed for the loading portion of all tests, both repetitive and non-repetitive in nature, which were done both in the presence of a Roadrain layer and without, using one of two different types of soil subbases. The characteristic curves, along with the stress range for which they were tested, are summarized in Tables 5.4 and 5.5 for tests conducted on the \#57 Limestone subbase and the A-2-4 soil subbase, respectively. 
Table 5.1: Suggested Values for Poisson's Ratio of Various Types of Soils [Source: Das, 1999]

\begin{tabular}{|l|c|}
\hline \multicolumn{1}{|c|}{ Soil Type } & $\begin{array}{c}\text { Poisson's Ratio, } \\
\boldsymbol{U}\end{array}$ \\
\hline Coarse Sand & $0.15-0.2$ \\
\hline Medium Loose Sand & $0.20-0.25$ \\
\hline Fine Sand & $0.25-0.30$ \\
\hline Sandy Silt and Silt & $0.30-0.35$ \\
\hline Saturated Clay (Undrained) & 0.5 \\
\hline Saturated Clay - Lightly Overconsolidated (Drained) & $0.2-0.4$ \\
\hline
\end{tabular}


Table 5.2: Loading Scenarios Used for All Plate Bearing Tests Using \#57 Limestone Subbase, In Order of Completion

\begin{tabular}{|c|c|c|}
\hline Test Designation & Roadrain Used? & Load Pattern Applied [lbs] \\
\hline Non-Repetitive & NO & $0 \rightarrow 350 \rightarrow 1850 \rightarrow 3350 \rightarrow 4850 \rightarrow 6350 \rightarrow 7850 \rightarrow 9350 \rightarrow 0[\mathrm{x} 3]$ \\
\hline Non-Repetitive & YES & $0 \rightarrow 350 \rightarrow 1850 \rightarrow 3350 \rightarrow 4850 \rightarrow 6350 \rightarrow 7850 \rightarrow 9350 \rightarrow 0[\mathrm{x} 3]$ \\
\hline Non-Repetitive, Recompacted & YES & $0 \rightarrow 350 \rightarrow 1850 \rightarrow 3350 \rightarrow 4850 \rightarrow 6350 \rightarrow 7850 \rightarrow 9350 \rightarrow 0[\mathrm{x} 4]$ \\
\hline Repetitive, Small Load & NO & $0 \rightarrow 2100 \rightarrow 0[\mathrm{x} 6]$ \\
\hline Repetitive, Medium Load & NO & $0 \rightarrow 24,600 \rightarrow 0[\mathrm{x} 6]$ \\
\hline Repetitive, Large Load & NO & $0 \rightarrow 50,000 \rightarrow 0[\mathrm{x} 4]$ \\
\hline Repetitive, Small Load & YES & $0 \rightarrow 450 \rightarrow 0[\mathrm{x} 6]$ \\
\hline Repetitive, Medium Load & YES & $0 \rightarrow 17,000 \rightarrow 0[\mathrm{x} 6]$ \\
\hline Repetitive, Large Load & YES & $0 \rightarrow 50,000 \rightarrow 0[\mathrm{x} 6]$ \\
\hline
\end{tabular}


Table 5.3: Loading Scenarios Used for All Plate Bearing Tests Using A-2-4 Soil Subbase, In Order of Completion

\begin{tabular}{|c|c|c|}
\hline Test Designation & Roadrain Used? & Load Pattern Applied [lbs] \\
\hline Non-Repetitive & NO & $0 \rightarrow 350 \rightarrow 1850 \rightarrow 3350 \rightarrow 4850 \rightarrow 6350 \rightarrow 7850 \rightarrow 9350 \rightarrow 0[\mathrm{x} 4]$ \\
\hline Non-Repetitive & YES & $0 \rightarrow 350 \rightarrow 1850 \rightarrow 3350 \rightarrow 4850 \rightarrow 6350 \rightarrow 7850 \rightarrow 9350 \rightarrow 0[\mathrm{x} 5]$ \\
\hline Non-Repetitive, Recompacted & YES & $0 \rightarrow 350 \rightarrow 1850 \rightarrow 3350 \rightarrow 4850 \rightarrow 6350 \rightarrow 7850 \rightarrow 9350 \rightarrow 0[\mathrm{x} 3]$ \\
\hline Repetitive, Small Load & NO & $0 \rightarrow 650 \rightarrow 0[\mathrm{x} 6]$ \\
\hline Repetitive, Small Load & YES & $0 \rightarrow 650 \rightarrow 0[\mathrm{x} 6]$ \\
\hline Repetitive, Medium Load & NO & $0 \rightarrow 16,000 \rightarrow 0[\mathrm{x} 6]$ \\
\hline Repetitive, Medium Load & YES & $0 \rightarrow 16,000 \rightarrow 0[\mathrm{x} 6]$ \\
\hline Repetitive, Large Load & NO & $0 \rightarrow 35,000 \rightarrow 0[\mathrm{x} 5]$ \\
\hline Repetitive, Large Load & YES & $0 \rightarrow 35,000 \rightarrow 0[\mathrm{x} 6]$ \\
\hline
\end{tabular}


Table 5.4: Characteristic Curves Obtained from Multiple Repetitive and Non-Repetitive Static Plate Bearing Tests Conducted on \#57 Limestone Subbases

\begin{tabular}{|c|c|c|c|c|}
\hline Test Designation & Roadrain Used? & Characteristic Equation & Load Duration & $\begin{array}{c}\text { Stress Range } \\
\text { (as Tested) }\end{array}$ \\
\hline Non-Repetitive & $\mathrm{NO}$ & $\sigma=2,124.8 u^{2}+110.0 u$ & Long-Term & Up to $13 p s i$ \\
\hline Non-Repetitive & YES & $\sigma=613.2 \mathrm{u}^{2}+53.6 \mathrm{u}$ & Long-Term & Up to $13 p s i$ \\
\hline Small Load, Repetitive & NO & $\sigma=2,179.2 u^{2}+131.1 u$ & Short-Term & Up to $3 p s i$ \\
\hline Small Load, Repetitive & YES & $\sigma=112.2 \mathrm{u}^{2}+25.8 \mathrm{u}$ & Short-Term & Up to $0.7 \mathrm{psi}$ \\
\hline Mid Load, Repetitive & NO & $\begin{aligned} \sigma= & (1.68 \mathrm{e} 6) \mathrm{u}^{4}-(2.27 \mathrm{e} 5) \mathrm{u}^{3} \\
& +(1.12 \mathrm{e} 4) \mathrm{u}^{2}-30 \mathrm{u}\end{aligned}$ & Short-Term & Up to $35 \mathrm{psi}$ \\
\hline Mid Load, Repetitive & YES & $\begin{aligned} \sigma= & (2.46 \mathrm{e} 5) \mathrm{u}^{4}-(5.21 \mathrm{e} 4) \mathrm{u}^{3} \\
& +(4.2 \mathrm{e} 3) \mathrm{u}^{2}-43 \mathrm{u}\end{aligned}$ & Short-Term & Up to $24 p s i$ \\
\hline Large Load, Repetitive & NO & $\begin{aligned} \sigma= & (3.98 \mathrm{e} 6) \mathrm{u}^{4}-(6.05 \mathrm{e} 5) \mathrm{u}^{3} \\
& +(3.04 \mathrm{e} 3) \mathrm{u}^{2}-305 \mathrm{u}\end{aligned}$ & Short-Term & Up to $70 \mathrm{psi}$ \\
\hline Large Load, Repetitive & YES & $\begin{aligned} \sigma= & (2.72 \mathrm{e} 5) \mathrm{u}^{4}-(5.55 \mathrm{e} 4) \mathrm{u}^{3} \\
& +(4.1 \mathrm{e} 3) \mathrm{u}^{2}-32 \mathrm{u}\end{aligned}$ & Short-Term & Up to $70 \mathrm{psi}$ \\
\hline
\end{tabular}


Table 5.5: Characteristic Curves Obtained from Multiple Repetitive and Non-Repetitive Static Plate Bearing Tests Conducted on A-2-4 Soil Subbases

\begin{tabular}{|c|c|c|c|c|}
\hline Test Designation & Roadrain Used? & Characteristic Equation & Load Duration & $\begin{array}{c}\text { Stress Range } \\
\text { (as Tested) }\end{array}$ \\
\hline Non-Repetitive & NO & $\sigma=286.3 \mathrm{u}^{2}+136.0 \mathrm{u}$ & Long-Term \\
\hline Non-Repetitive & YES & $\sigma=687.5 \mathrm{u}^{2}$ & Long-Term $13 p s i$ \\
\hline Small Load, Repetitive & NO & $\sigma=922.9 \mathrm{u}^{2}+40.5 \mathrm{u}$ & Short-Term & Up to 13psi $1 \mathrm{psi}$ \\
\hline Small Load, Repetitive & YES & $\sigma=357.3 \mathrm{u}^{2}+13.2 \mathrm{u}$ & Short-Term & Up to 1psi \\
\hline Mid Load, Repetitive & NO & $\sigma=-124,437 \mathrm{u}^{4}+3,539 \mathrm{u}^{2}$ & Short-Term & Up to 25psi \\
\hline Mid Load, Repetitive & YES & $\sigma=30,757 \mathrm{u}^{4}+599.9 \mathrm{u}^{2}$ & Short-Term & Up to 25psi \\
\hline Large Load, Repetitive & NO & $\sigma=155,785 \mathrm{u}^{4}+688.3 \mathrm{u}^{2}$ & Short-Term & Up to 50psi \\
\hline Large Load, Repetitive & YES & $\sigma=21,195 \mathrm{u}^{4}+666.5 \mathrm{u}^{2}$ & Short-Term & Up to 50psi \\
\hline
\end{tabular}




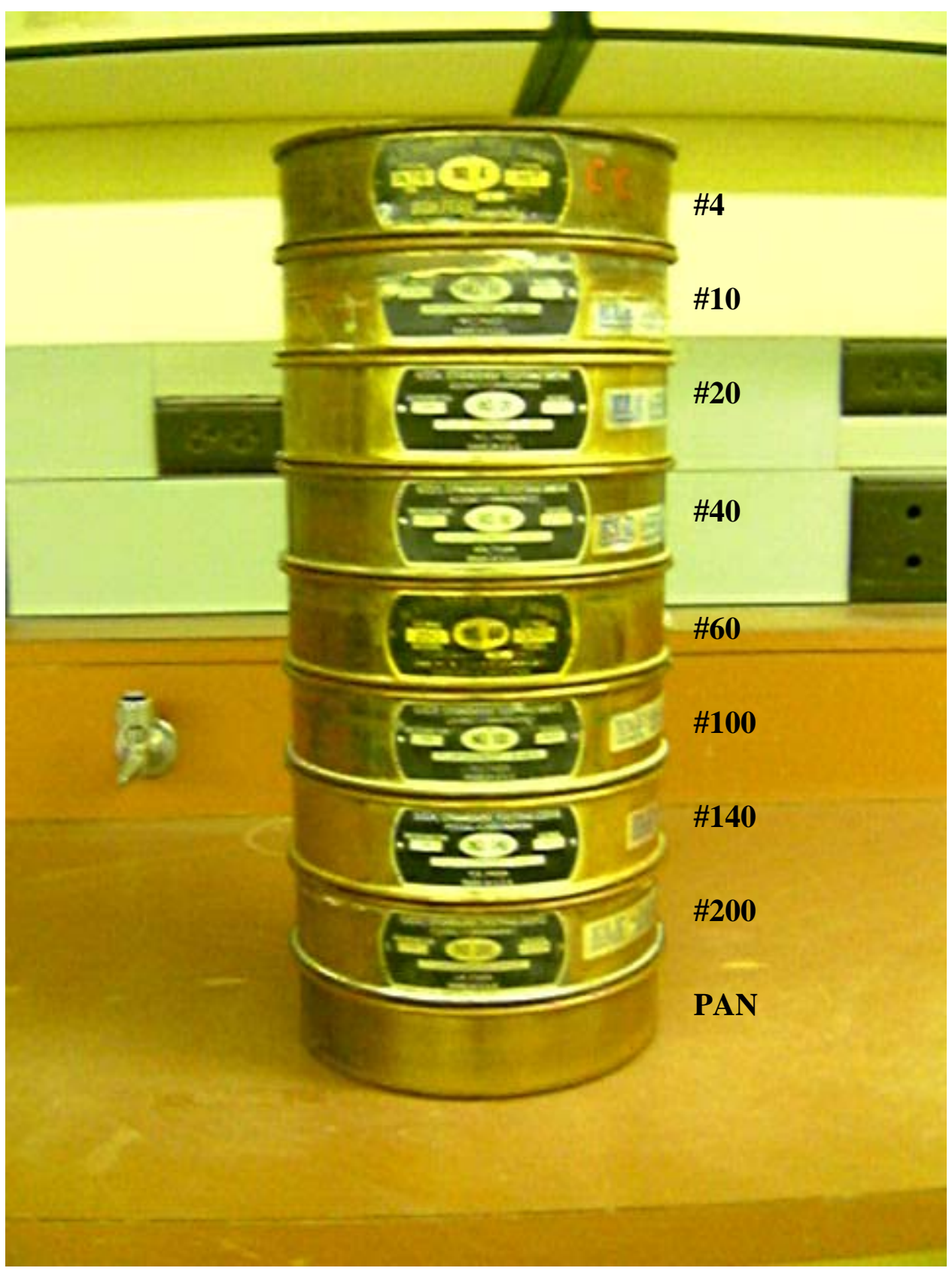

Figure 5.1: Sieves Used for Sieve Analysis of Excavated Soil (U.S. Standard Sieve Numbers) 


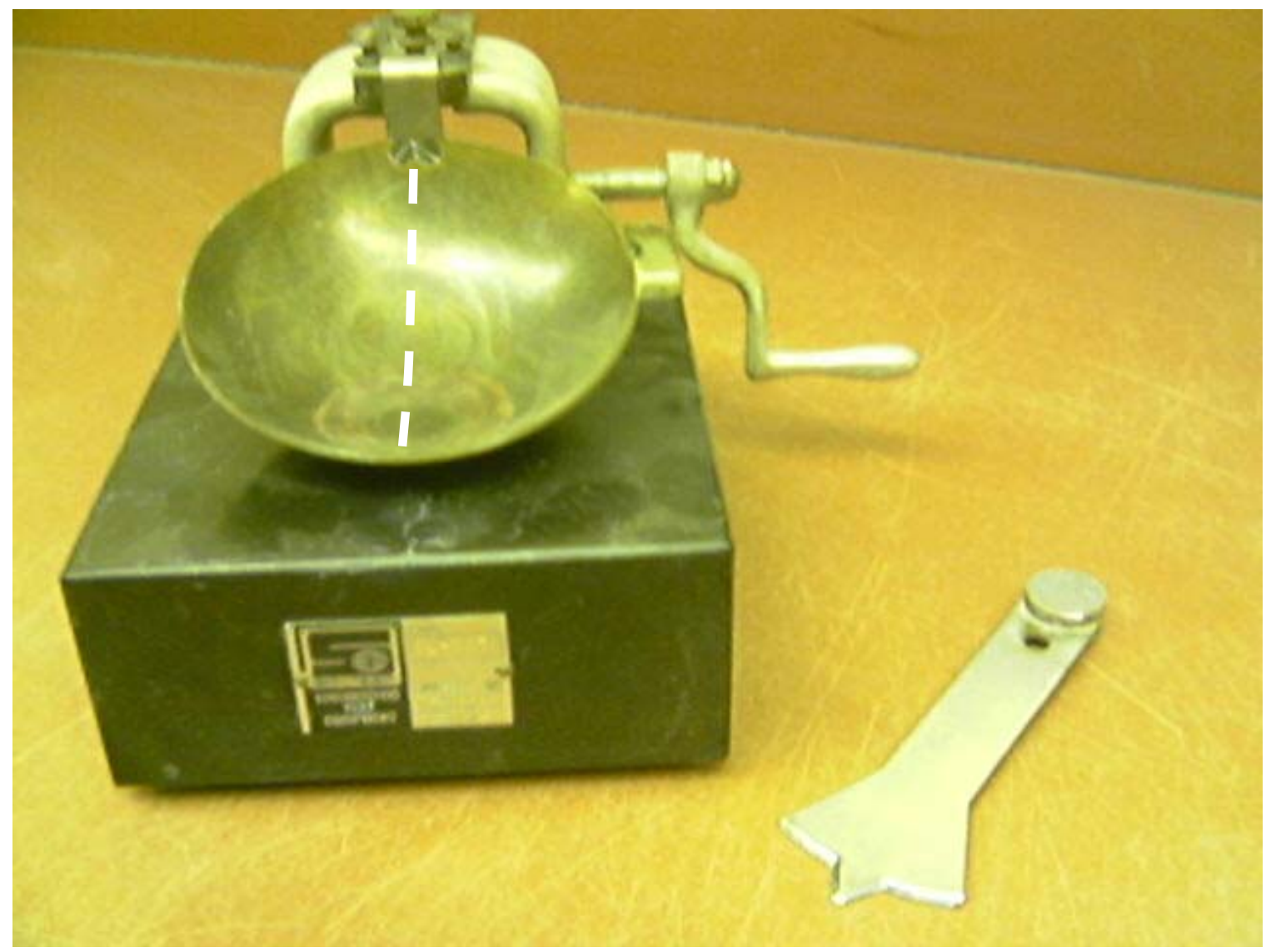

Figure 5.2: Liquid Limit Device (Left) and Grooving Tool (Right) Used for Liquid Limit Test, and Approximate Orientation of Groove as Formed in Sample Using the Grooving Tool 


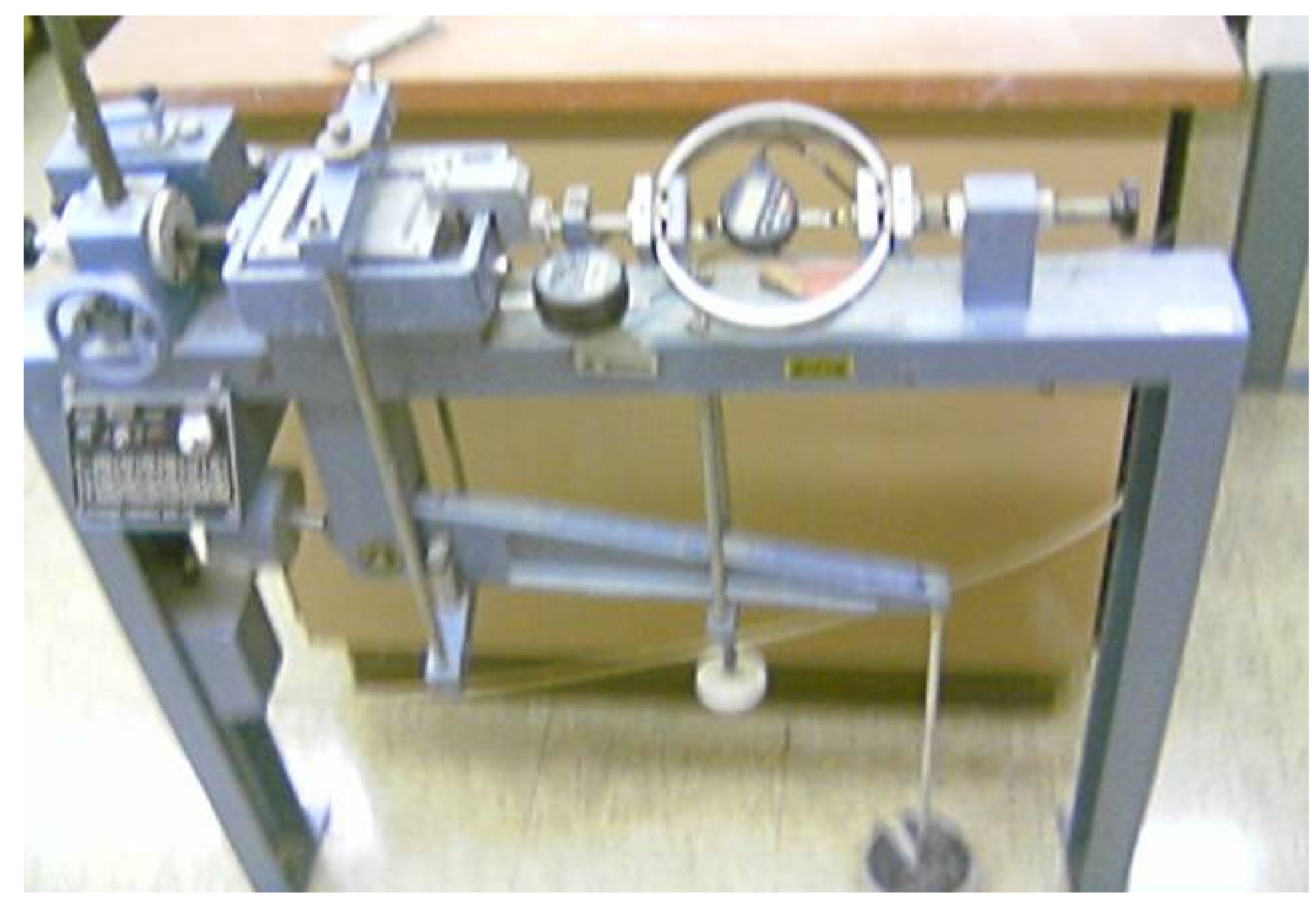

Figure 5.3: Direct Shear Test Setup Used for Soil Classification for Excavated Soil of Plate Bearing Tests 


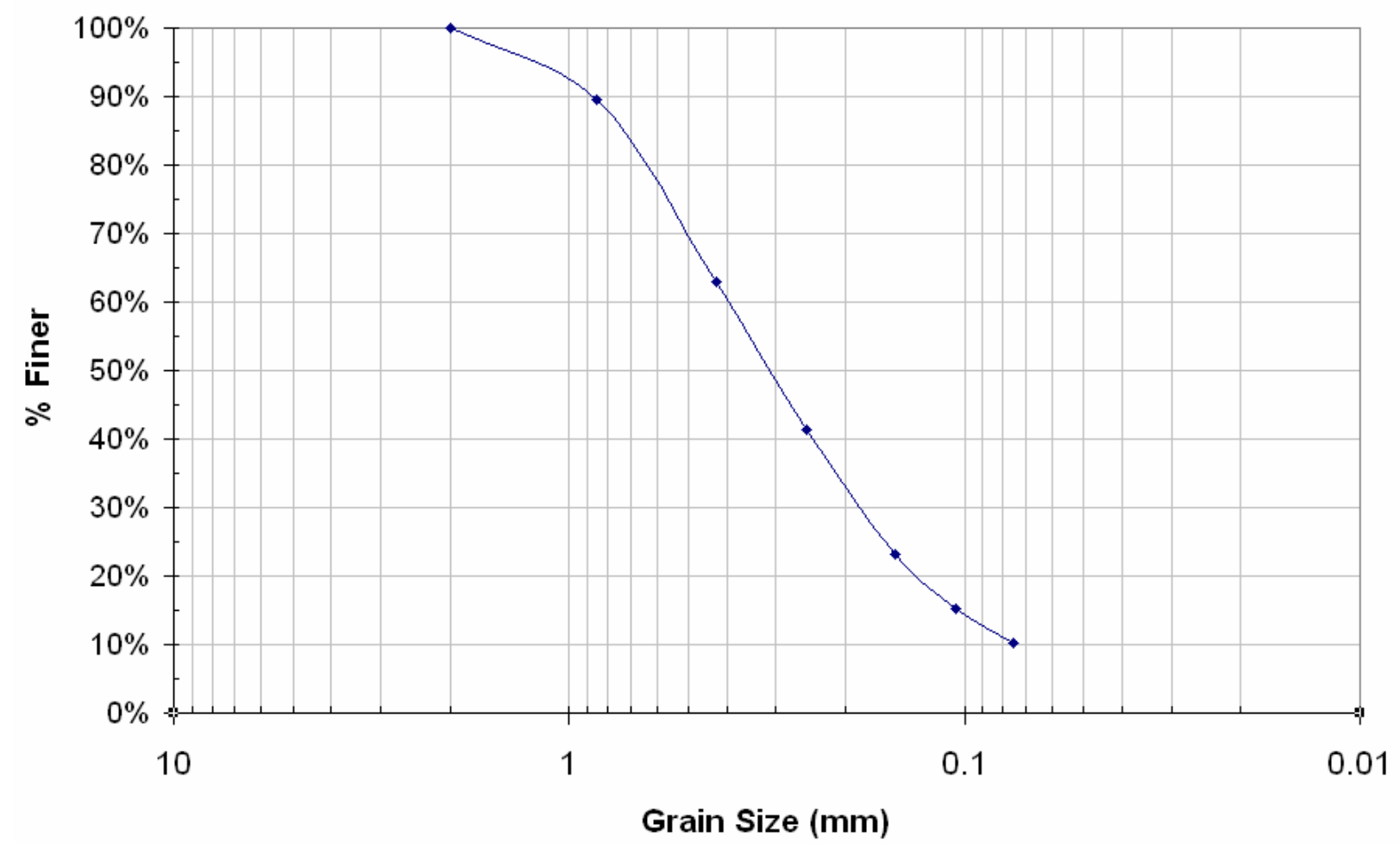

Figure 5.4: Grain Sizes Obtained from the Sieve Analysis of Excavated Soil Used as a Subbase for Plate Bearing Tests

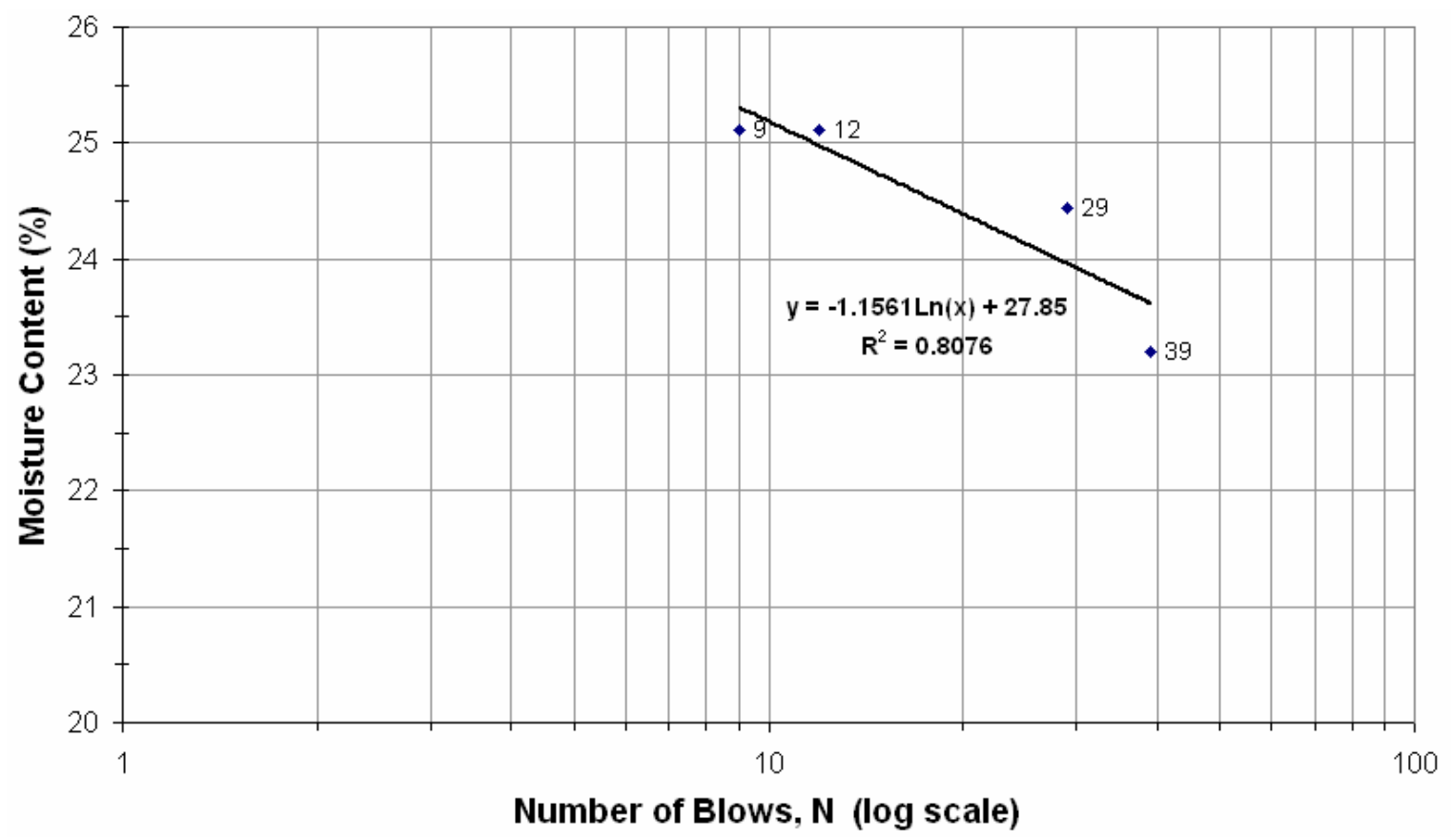

Figure 5.5: Flow Curve for Liquid Limit Determination of Excavated Soil Used as a Subbase for Plate Bearing Tests 


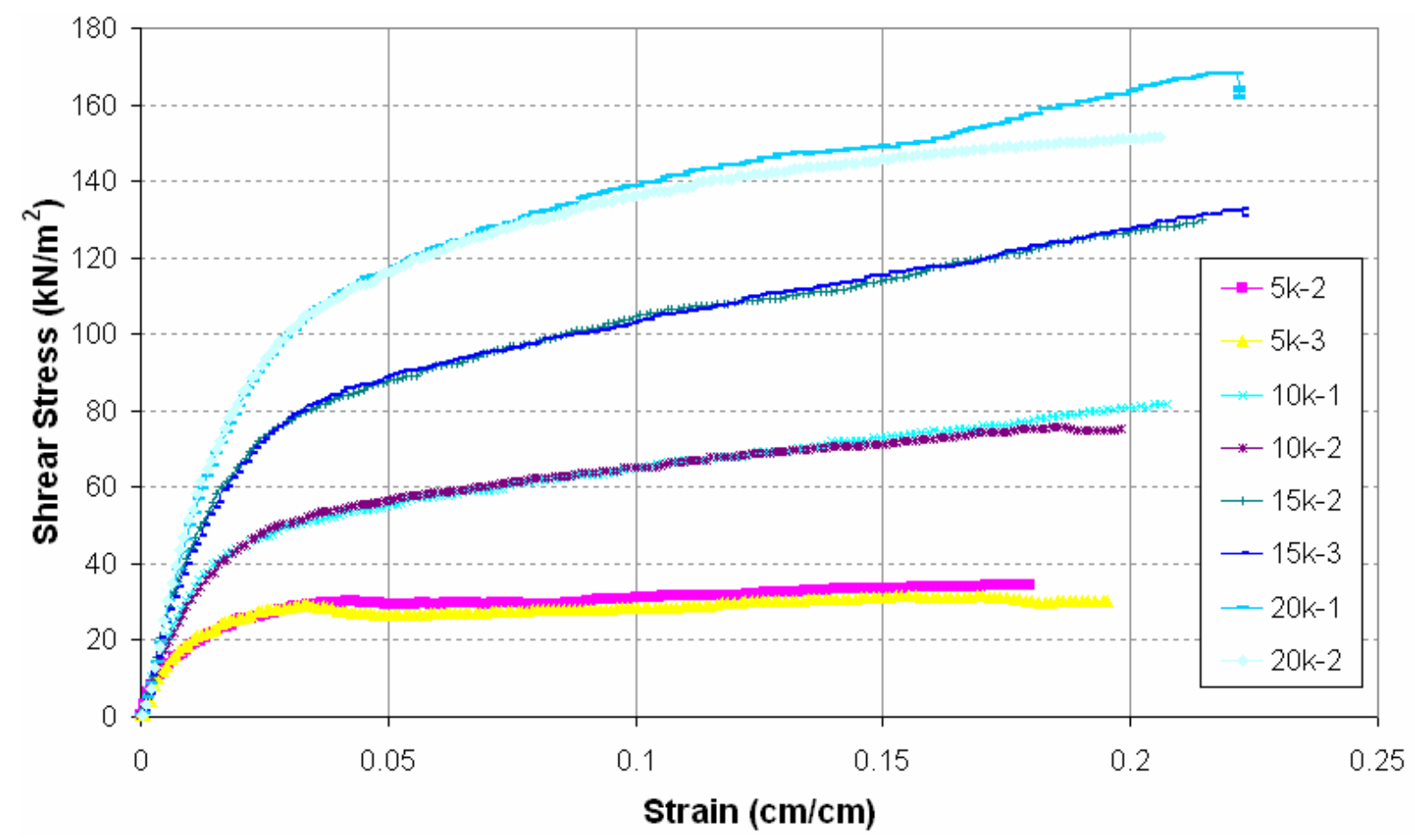

Figure 5.6: Shear Stress vs. Shear Strain Curve from Direct Shear Testing of Excavated Soil Used as a Subbase for Plate Bearing Tests

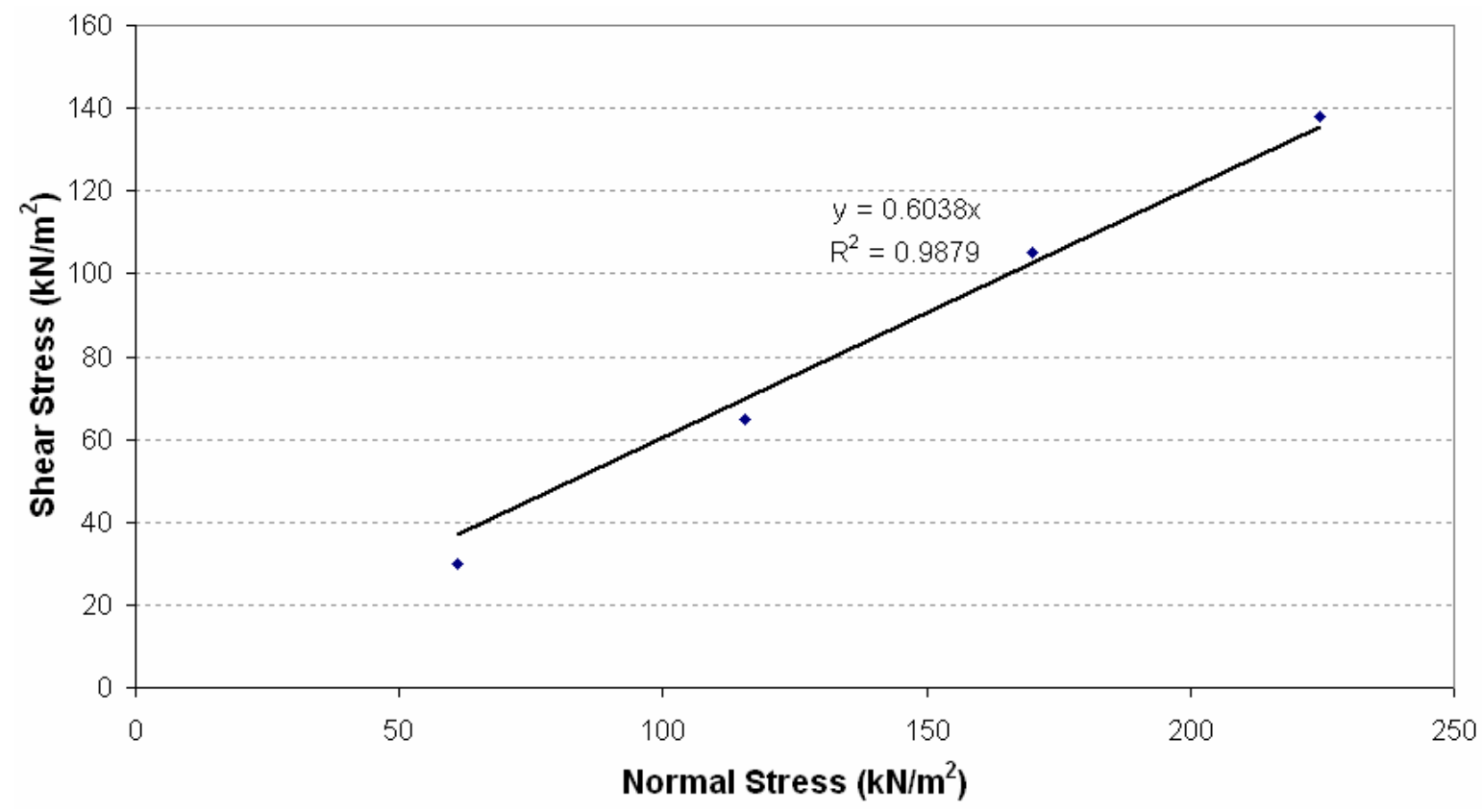

Figure 5.7: Shear Stress (@10\% Strain) vs. Normal Stress from Direct Shear Testing of Excavated Soil Used as a Subbase for Plate Bearing Tests 


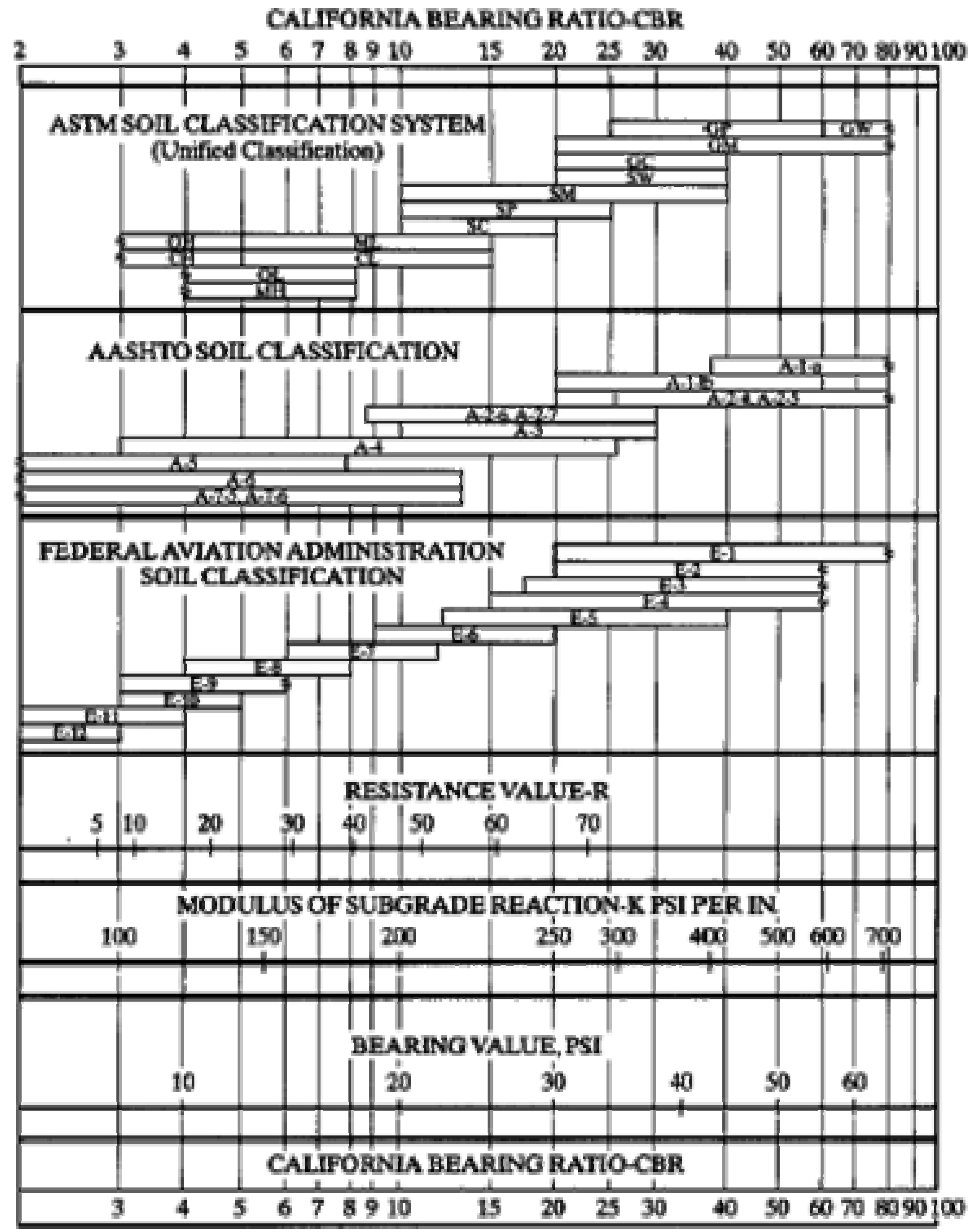

Figure 5.8: Approximate Interrelationships of Soil Classifications and Bearing Values [Source: PCA, 1992] 


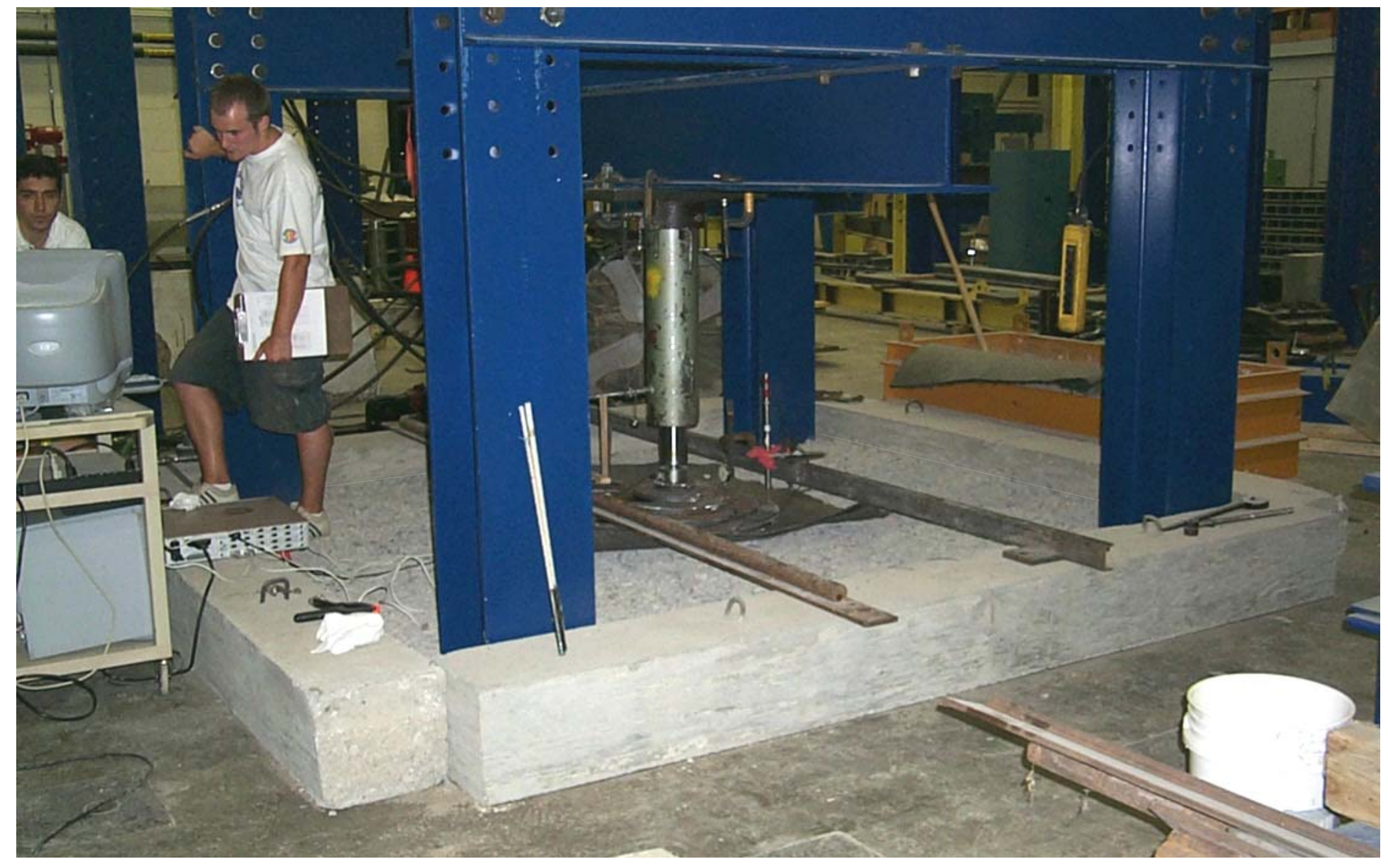

Figure 5.9: Overall View of Plate Bearing Test Setup 


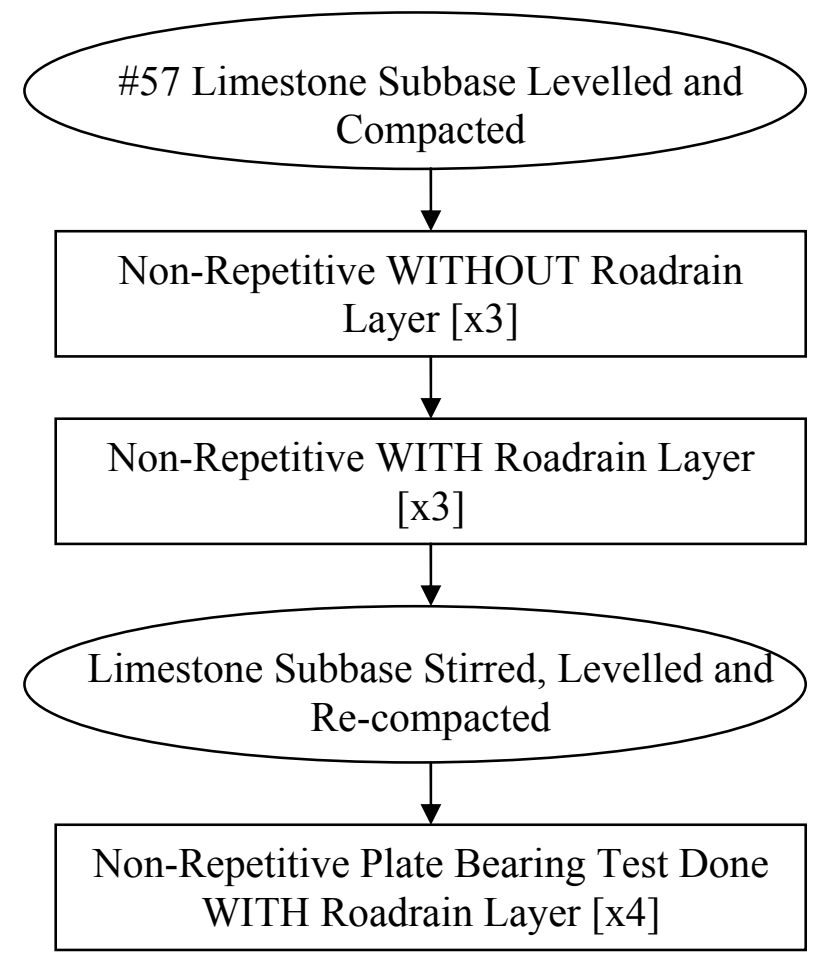

Figure 5.10: Flow Chart for Subbase Preparation and Testing History of Non-Repetitive Tests Utilizing \#57 Limestone Aggregate Subbases

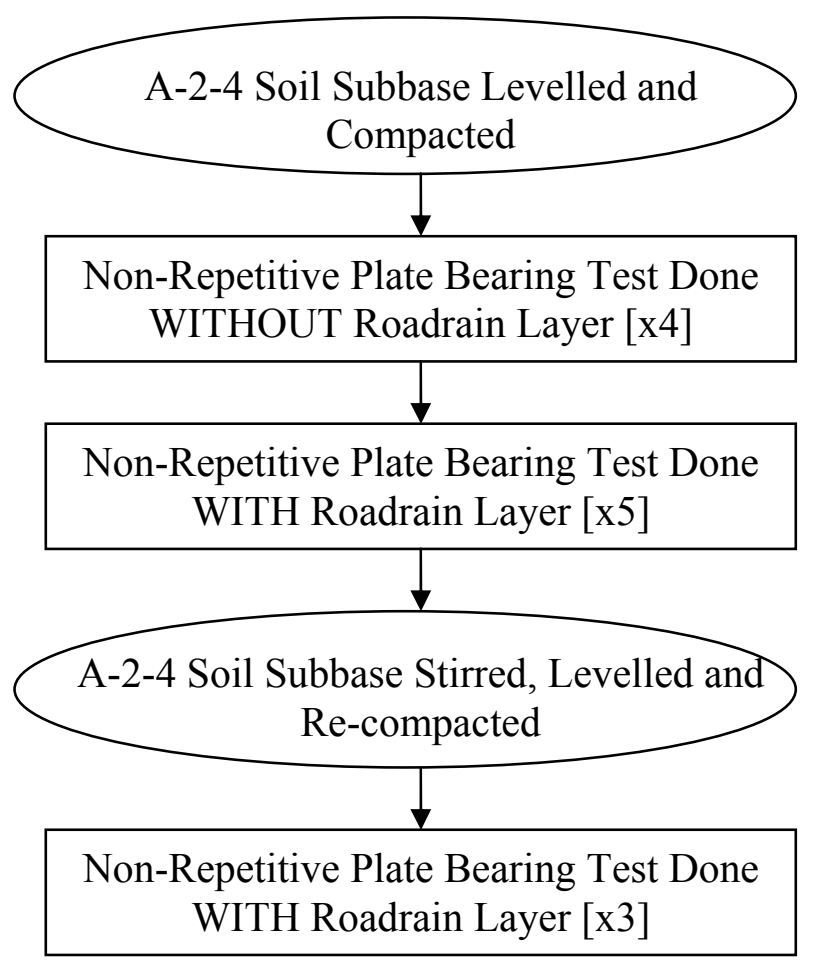

Figure 5.11: Flow Chart for Subbase Preparation and Testing History of Non-Repetitive Tests Utilizing A-2-4 Soil Subbases 


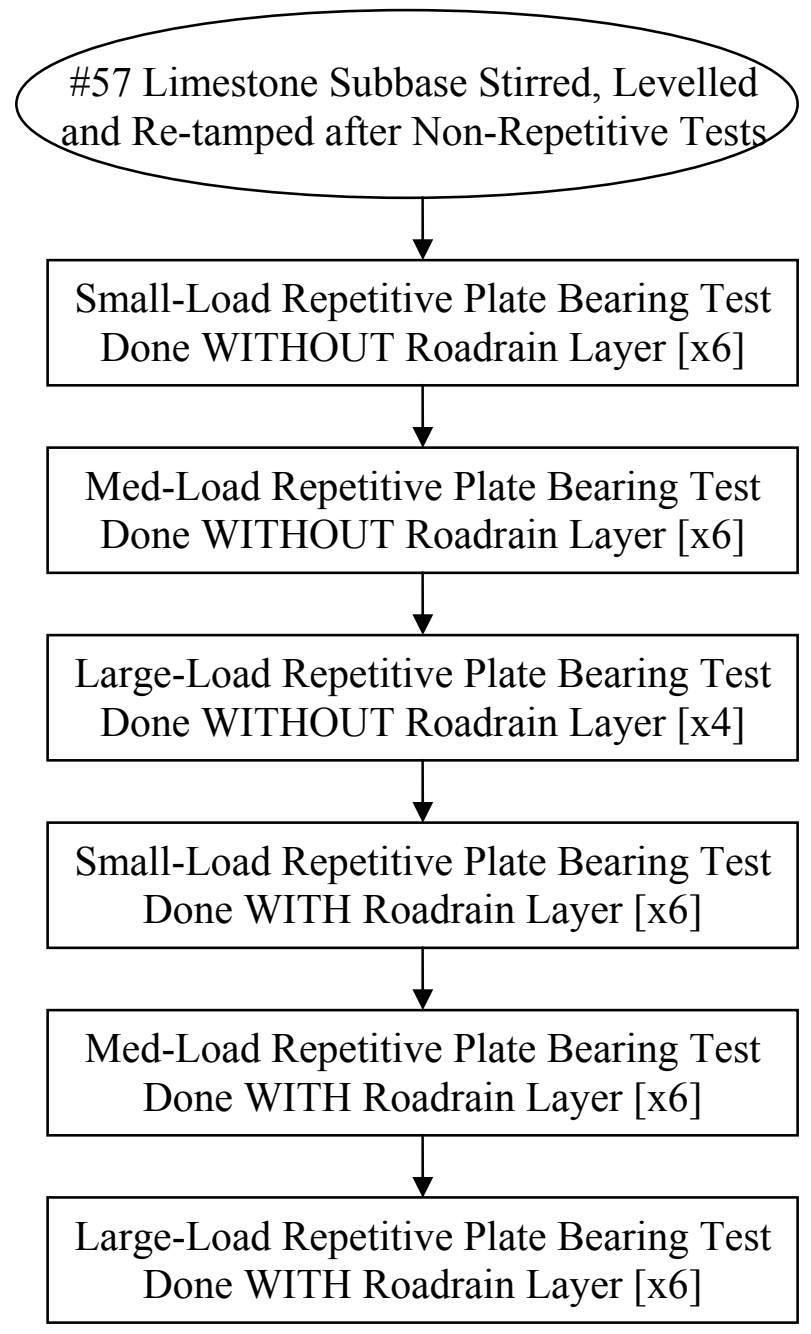

Figure 5.12: Flow Chart for Subbase Preparation and Testing History of Repetitive Tests Utilizing \#57 Limestone Aggregate Subbases 


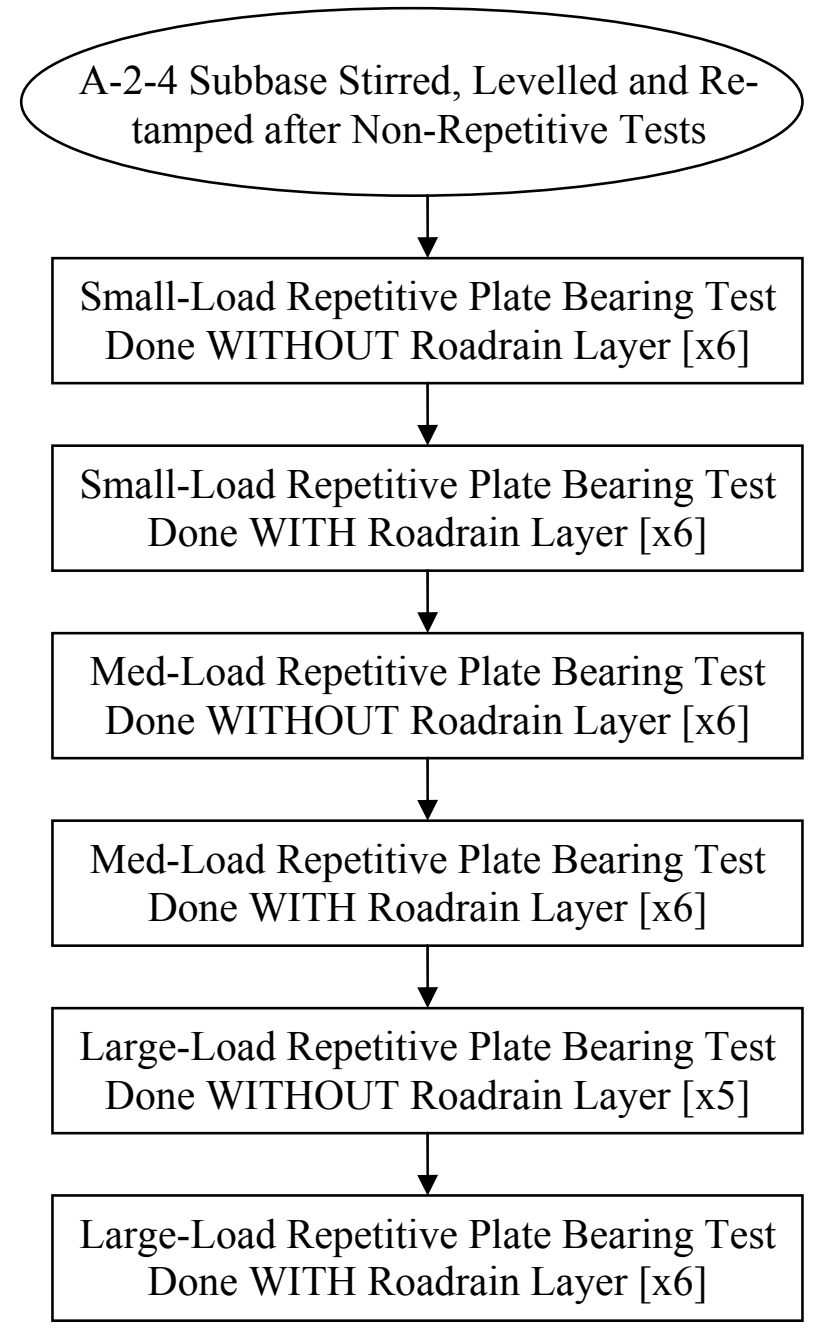

Figure 5.13: Flow Chart for Subbase Preparation and Testing History of Repetitive Tests Utilizing A-2-4 Subbases 


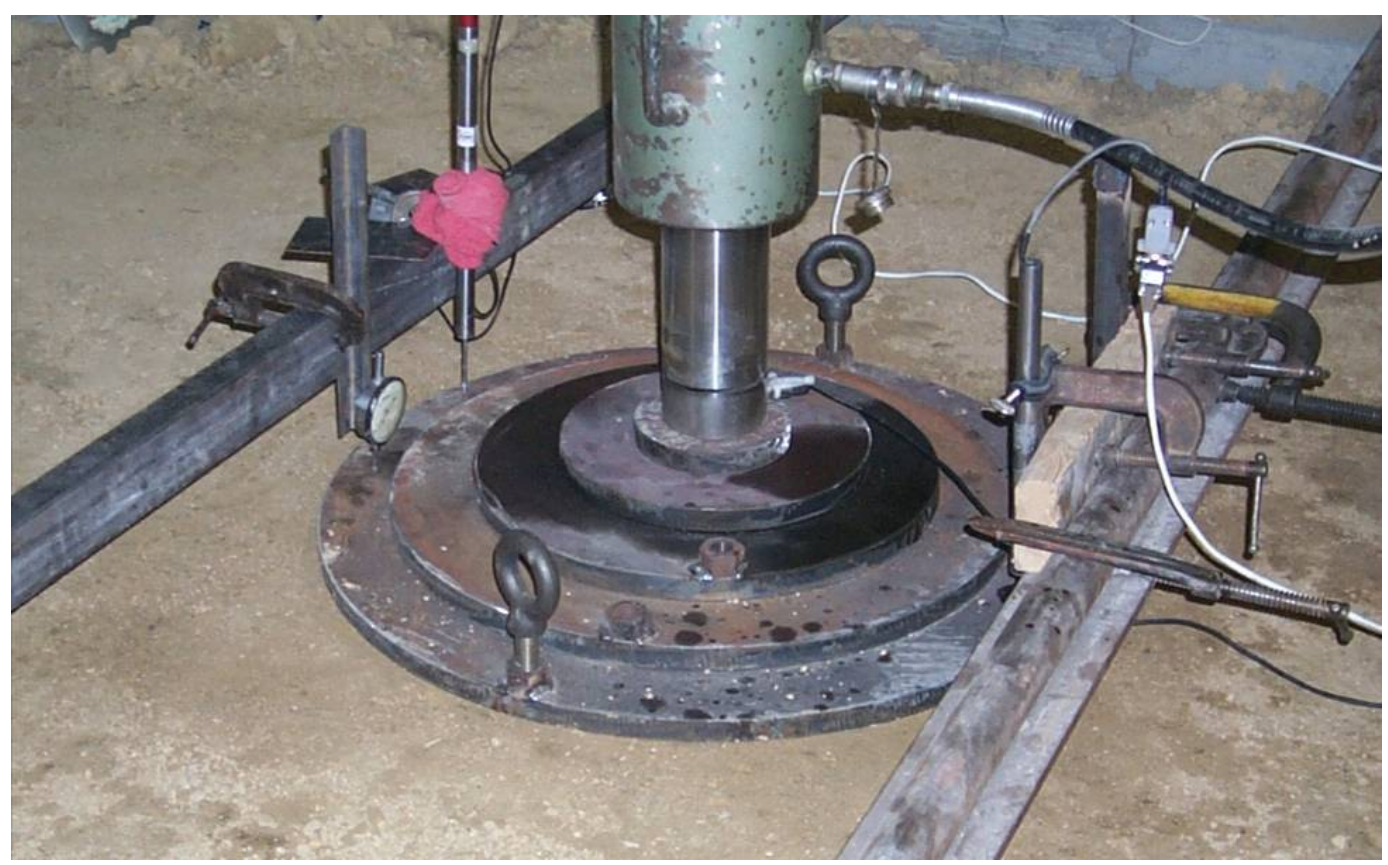

(a)

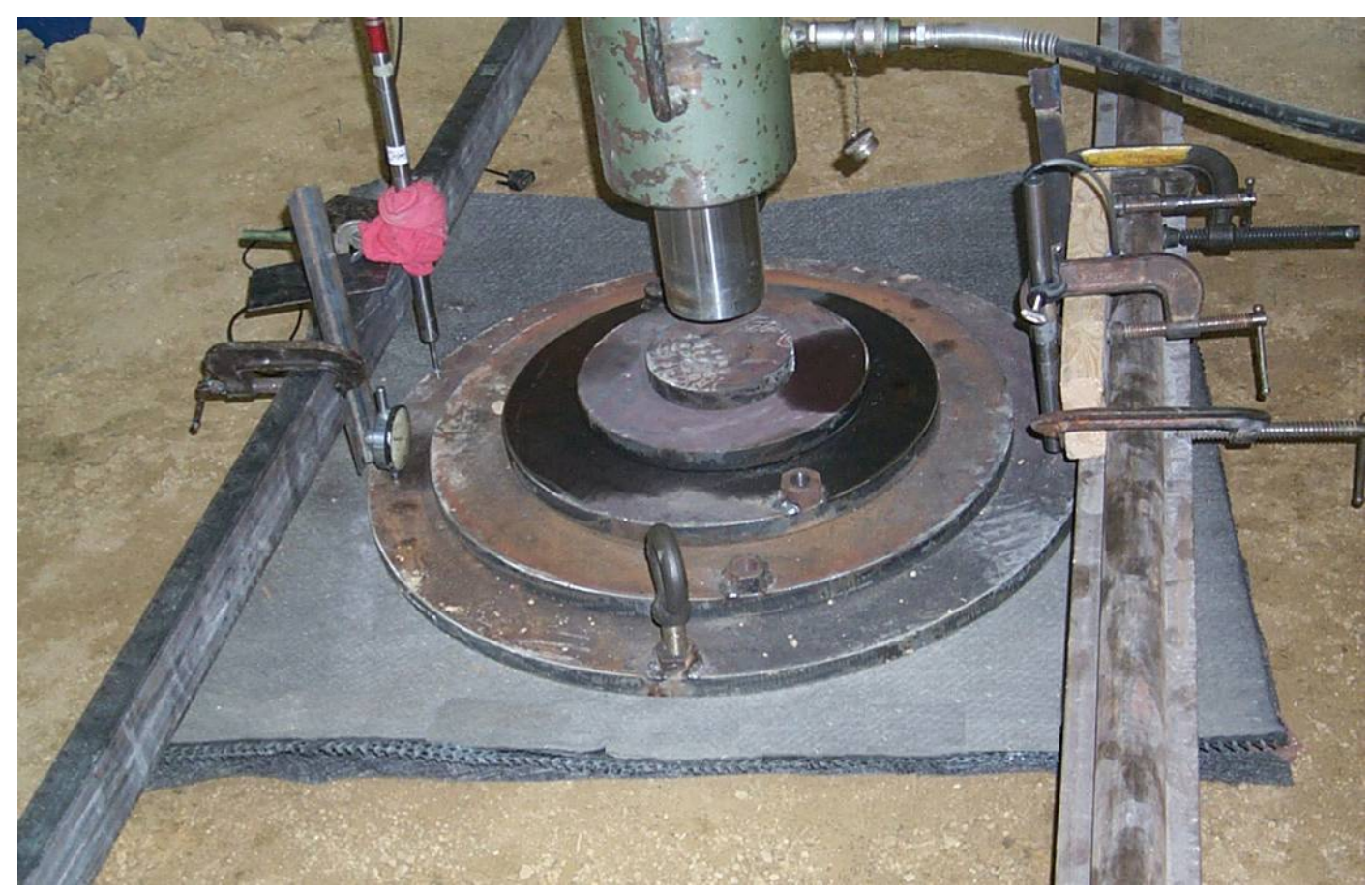

(b)

Figure 5.14: Picture of Sample, Loading Plates and Instrumentation Setup for Test of an A-2-4 Soil Subbase (a) without Roadrain, and (b) with Roadrain (shown w/o Load Cell) 


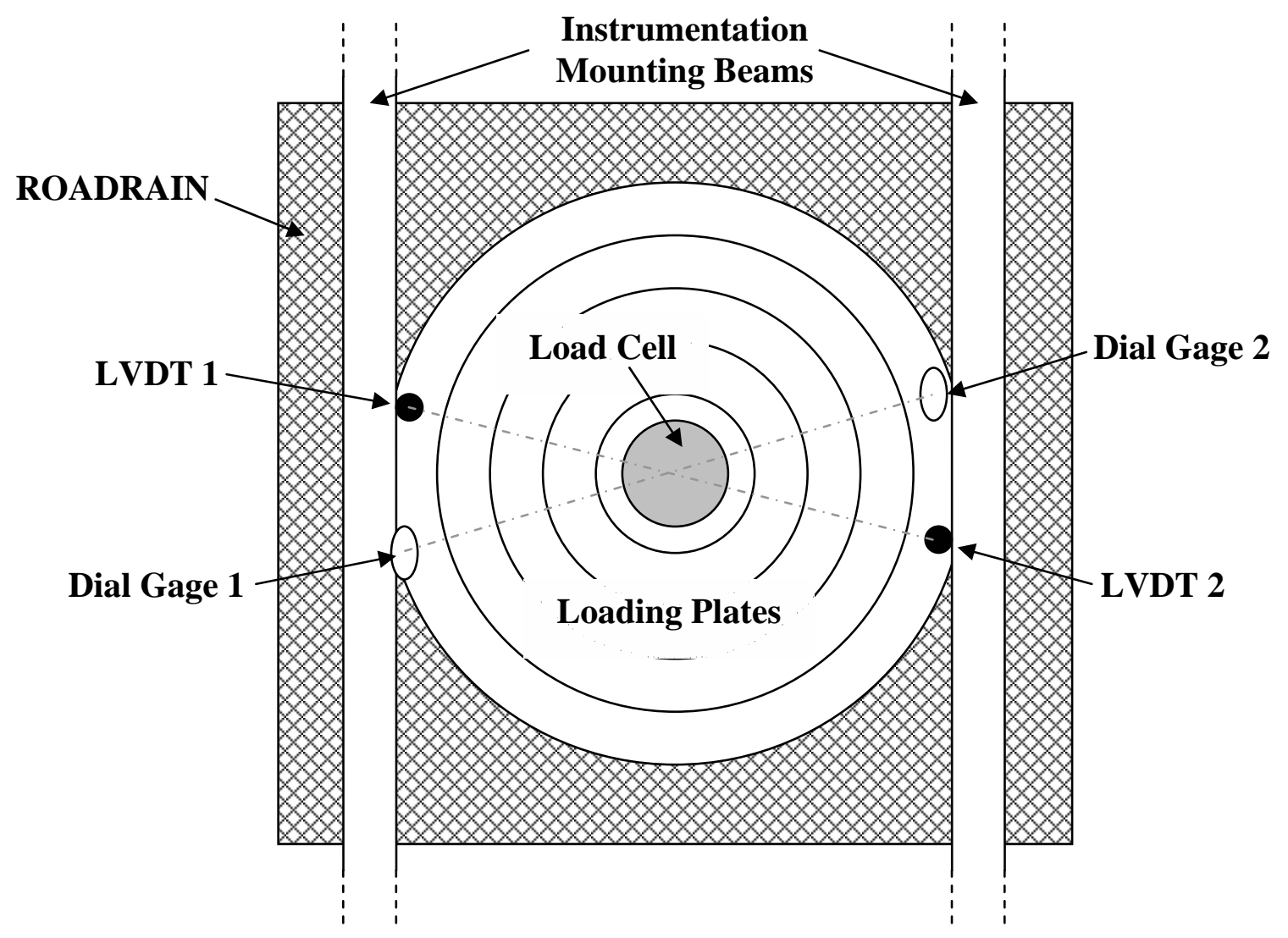

Figure 5.15: Schematic Overhead View of Instrumentation Placement (Not to Scale)

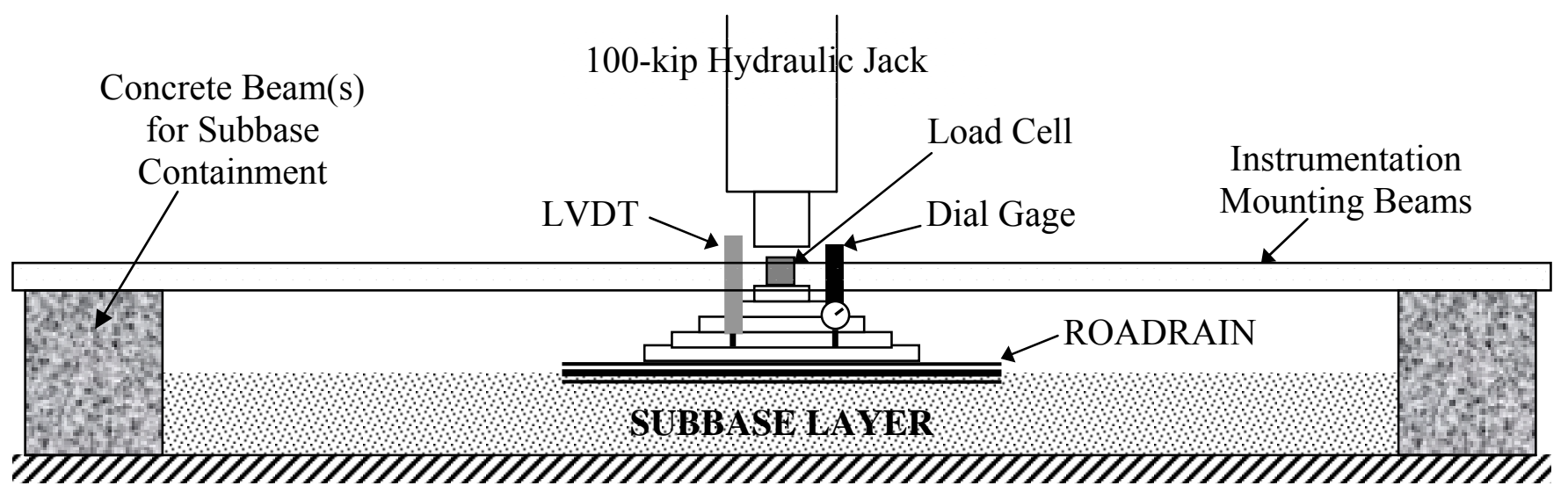

Figure 5.16: Schematic Side View of Instrumentation Placement (Not to Scale) 


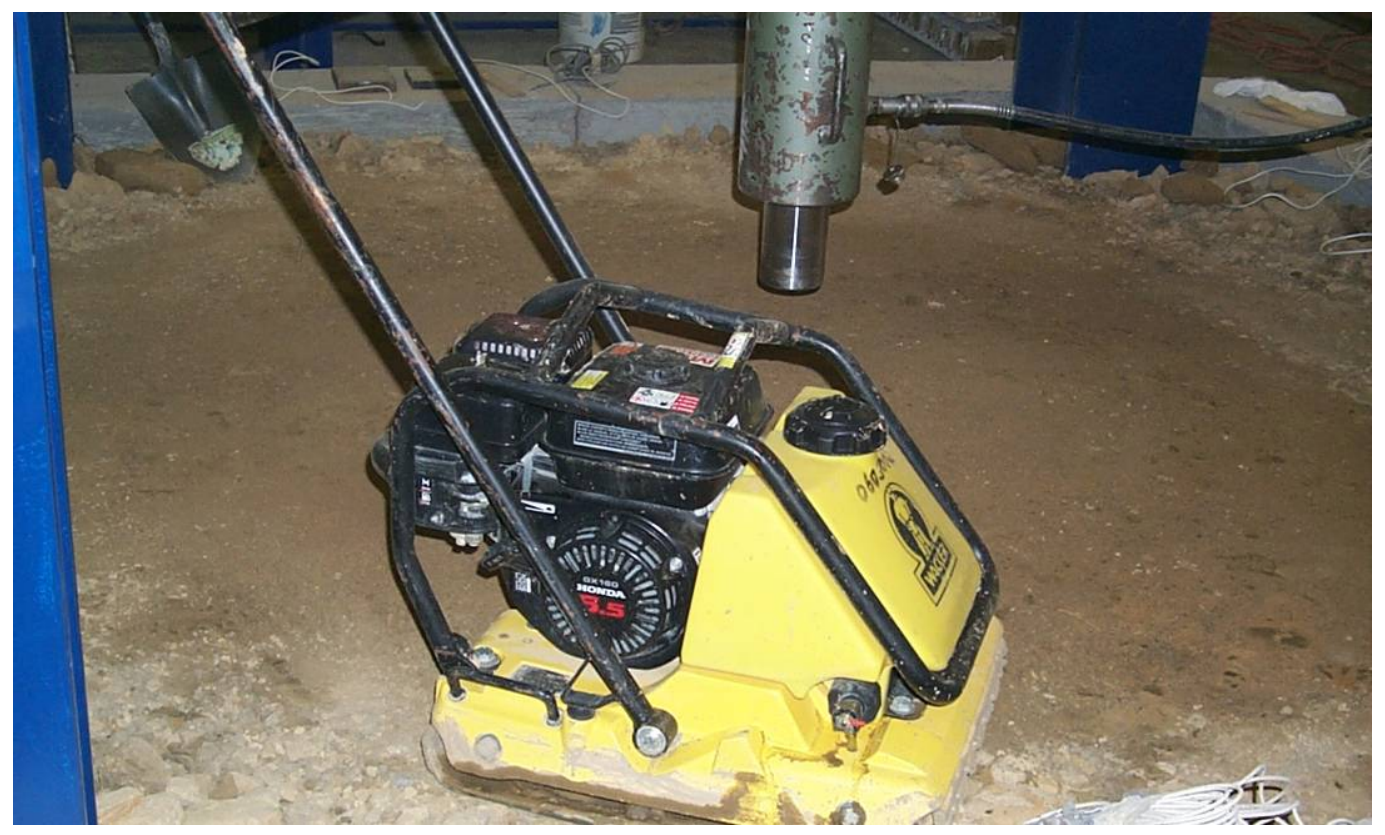

Figure 5.17: Plate Tamper Used for Subbase Compaction, Plate Bearing Tests 


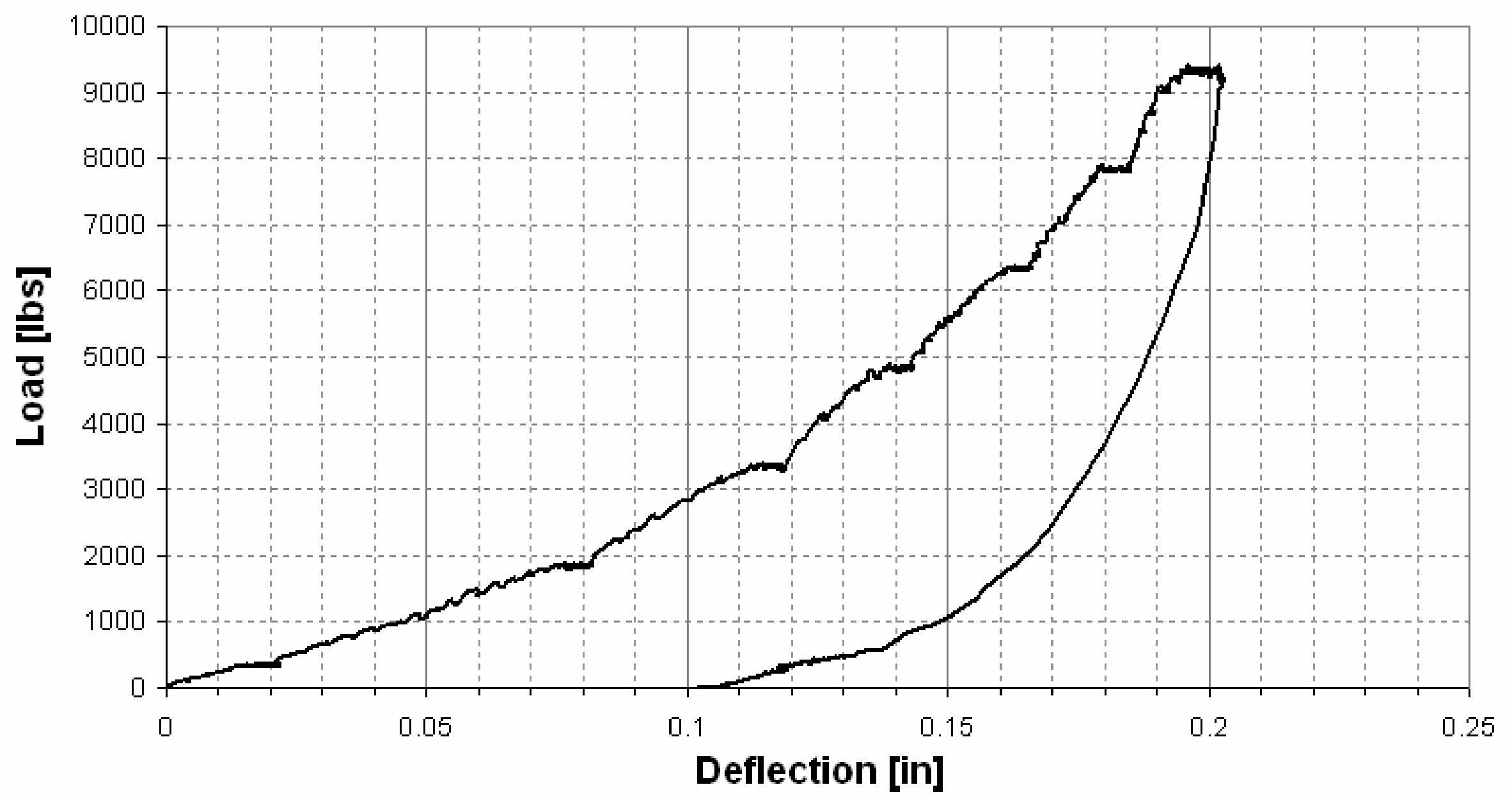

Figure 5.18: Sample Load-Deflection Curve from Non-Repetitive Plate Bearing Tests

Obtained from the First Loading of a Compacted \#57 Limestone Aggregate Subbase below a Previously Untested Roadrain Layer 


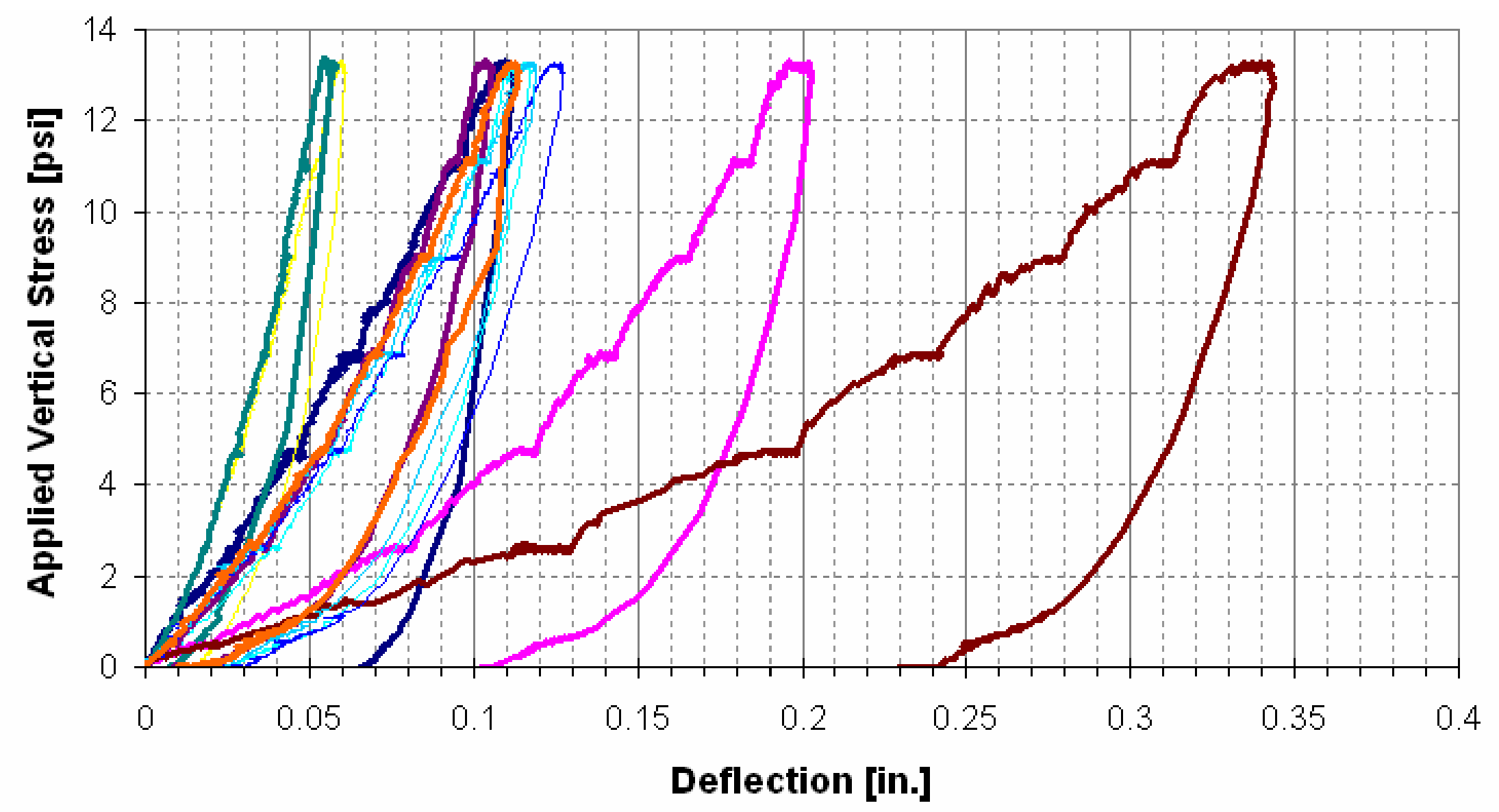

$\begin{array}{lll}\text { - AGG alone 1 } & \text { AGG alone 2 } & \text { - AGG alone } 3 \\ \text { - \#57Agg w/Roadrain 1 } & \text { - \#57Agg w/Roadrain 2 } & \text { - \#57Agg w/Roadrain 3 } \\ \text { - Recompacted Agg w/Roadrain 1 } & \text { - Recompacted Agg w/Roadrain 2 } & \text { - Recompacted Agg w/Roadrain 3 } \\ \text { Recompacted Agg w/Roadrain 4 } & & \end{array}$

Figure 5.19: Stress-Deflection Curves from All Non-Repetitive Plate Bearing Tests Using \#57 Limestone Aggregate Subbases 


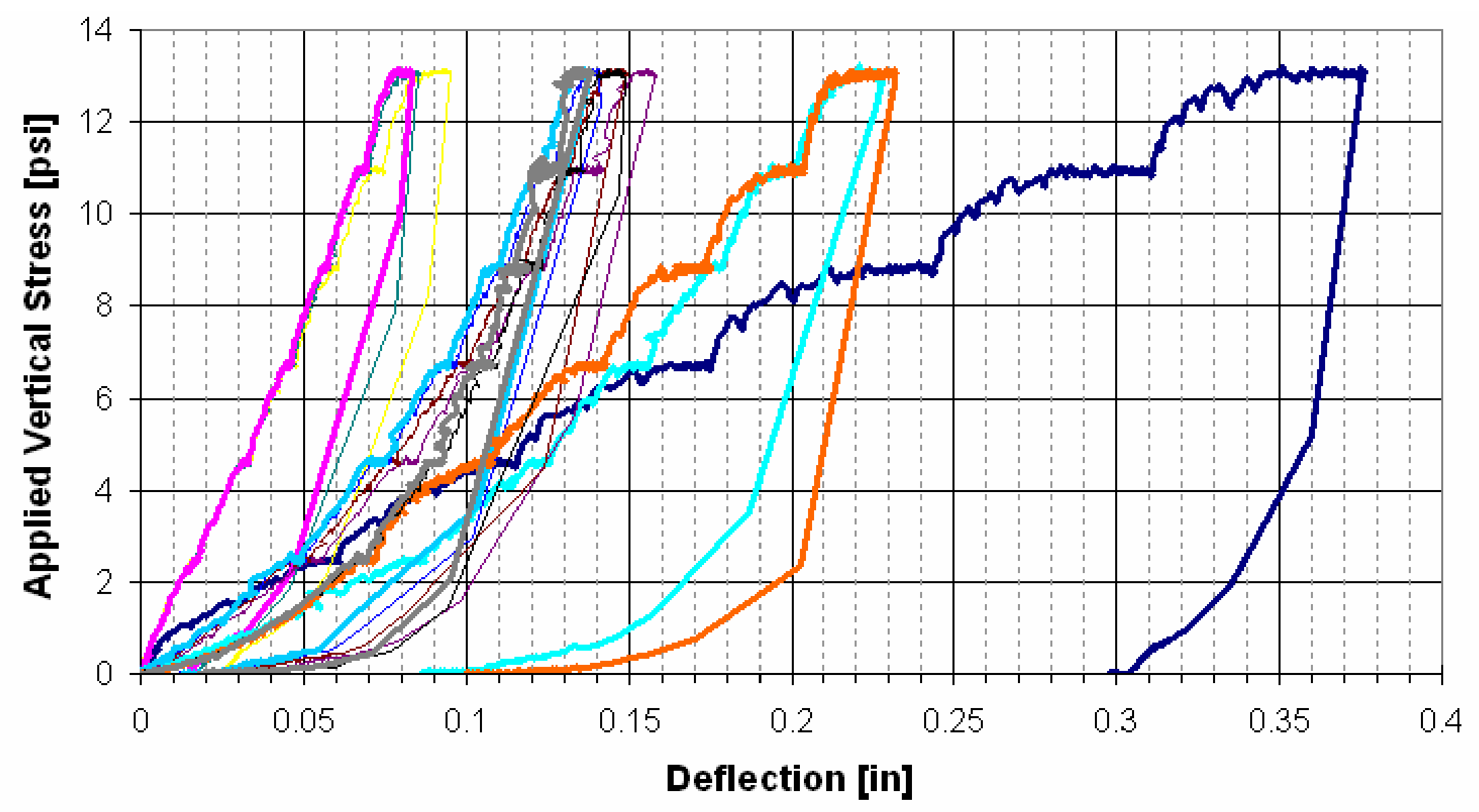

-A-2-4 Soil Alone 1

-A-2-4 Soil Alone 4 A-2-4 Soil Alone 2

- A-2-4 Soil w/Roadrain 1

A-2-4 Soil Alone 3

A-2-4 Soil w/Roadrain 3

- A-2-4 Soil w/Roadrain 4

- A-2-4 Soil w/Roadrain 2

- Recompacted A-2-4 w/Roadrain 1

- Recompacted A-2-4 w/Roadrain 2

-A-2-4 Soil w/Roadrain 5

- Recompacted A-2-4 W/Roadrain 3

Figure 5.20: Stress-Deflection Curves from All Non-Repetitive Plate Bearing Tests Using A-2-4 Soil Subbases 


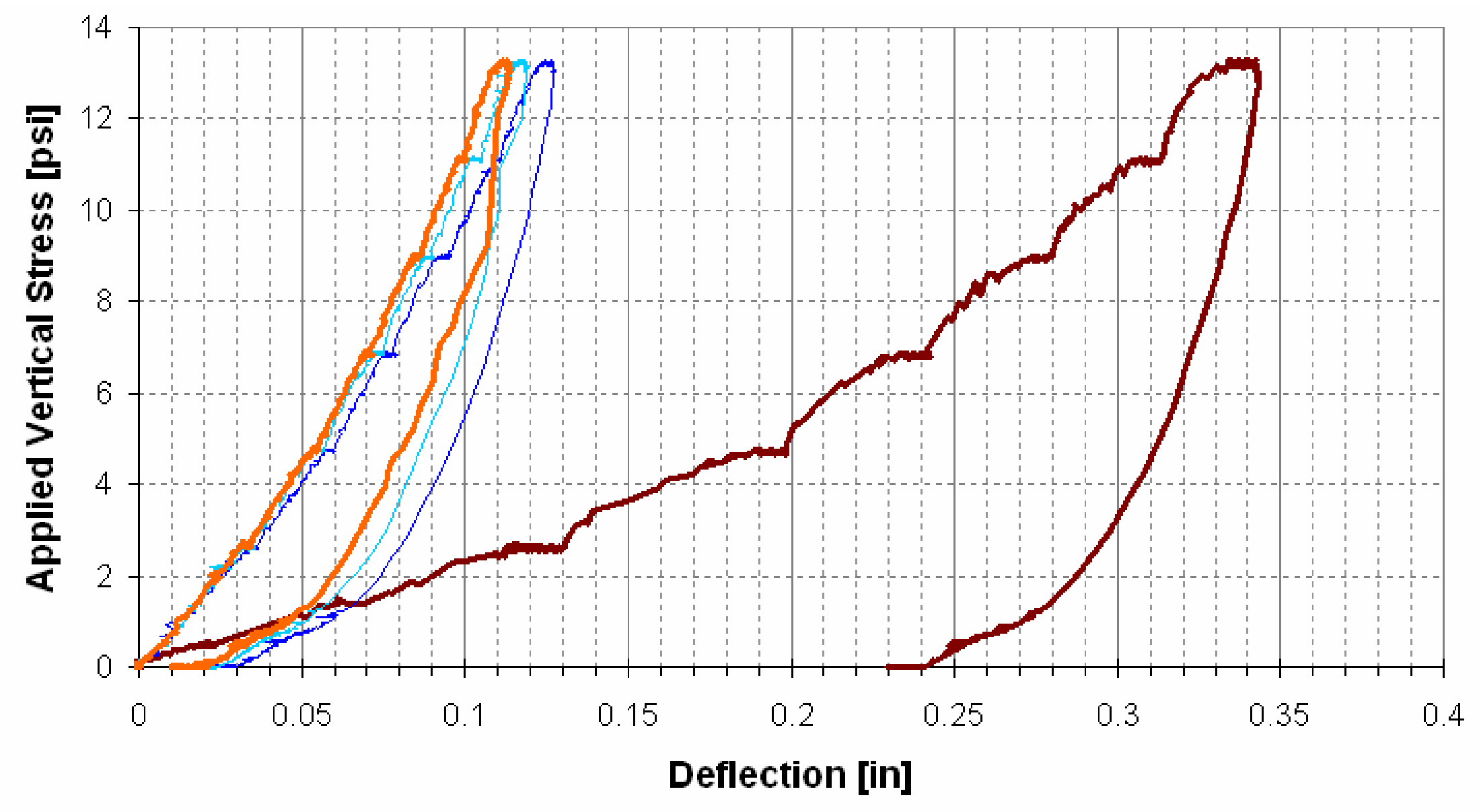

\begin{tabular}{|ll|}
\hline - Recompacted \#57Agg w/Roadrain 1 & - Recompacted \#57Agg w/Roadrain 2 \\
- Recompacted \#57Agg w/Roadrain 3 & -Recompacted \#57Agg w/Roadrain 4 \\
\hline
\end{tabular}

Figure 5.21: Stress-Deflection Curves from All Trials of the Non-Repetitive Plate Bearing Tests of a Recompacted \#57 Limestone Aggregate Subbase plus a Roadrain Layer 


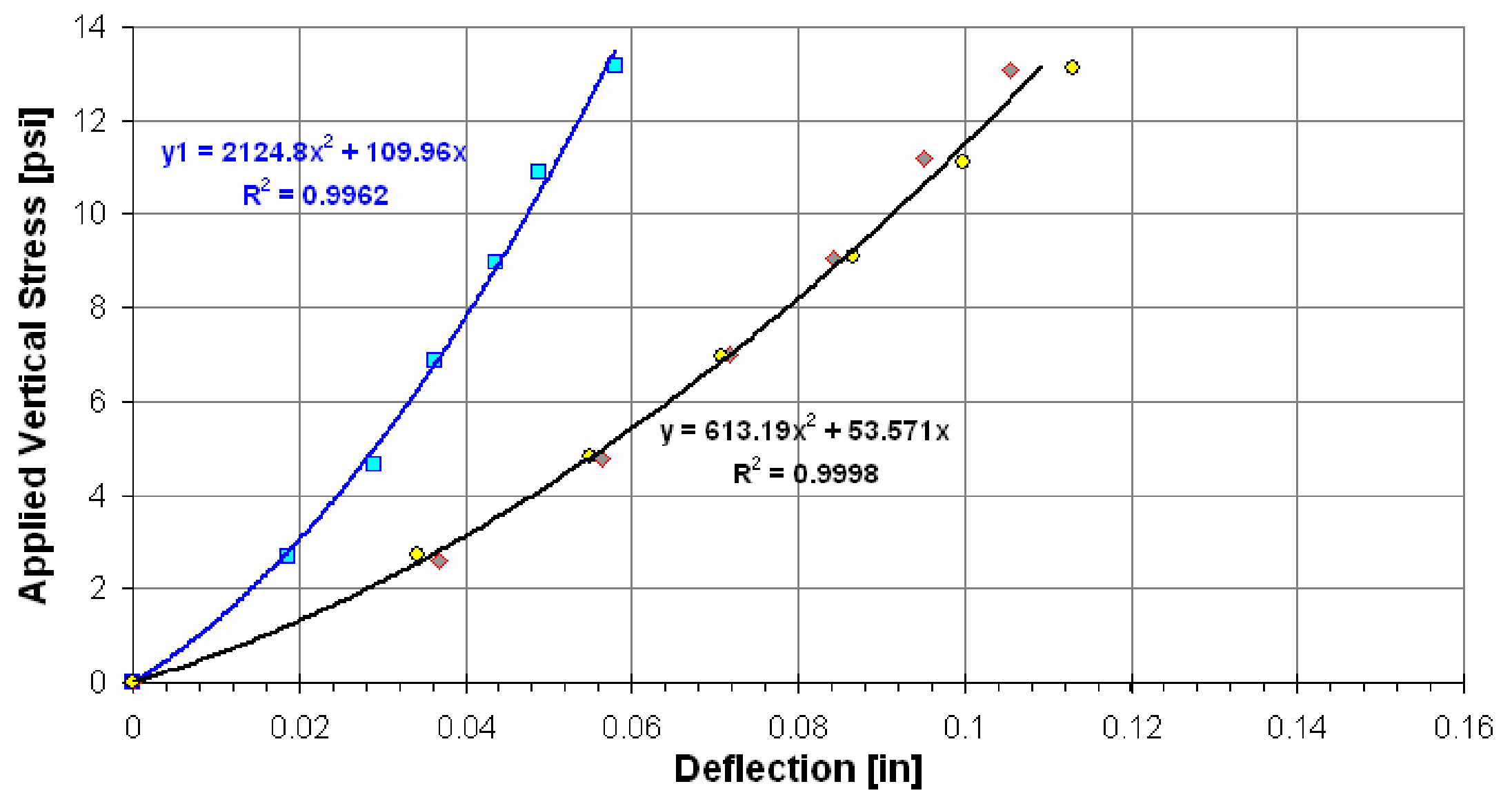

口 AGG. ALONE 3

औ57Agg w/Roadrain 3

- Recompacted Agg w/Roadrain 4

- Poly. (Average both Agg+Roadrain Tests)

Figure 5.22: Stress-Deflection Trendlines from Final Trials of All Non-Repetitive Plate Bearing Tests Using \#57 Limestone Aggregate Subbases 


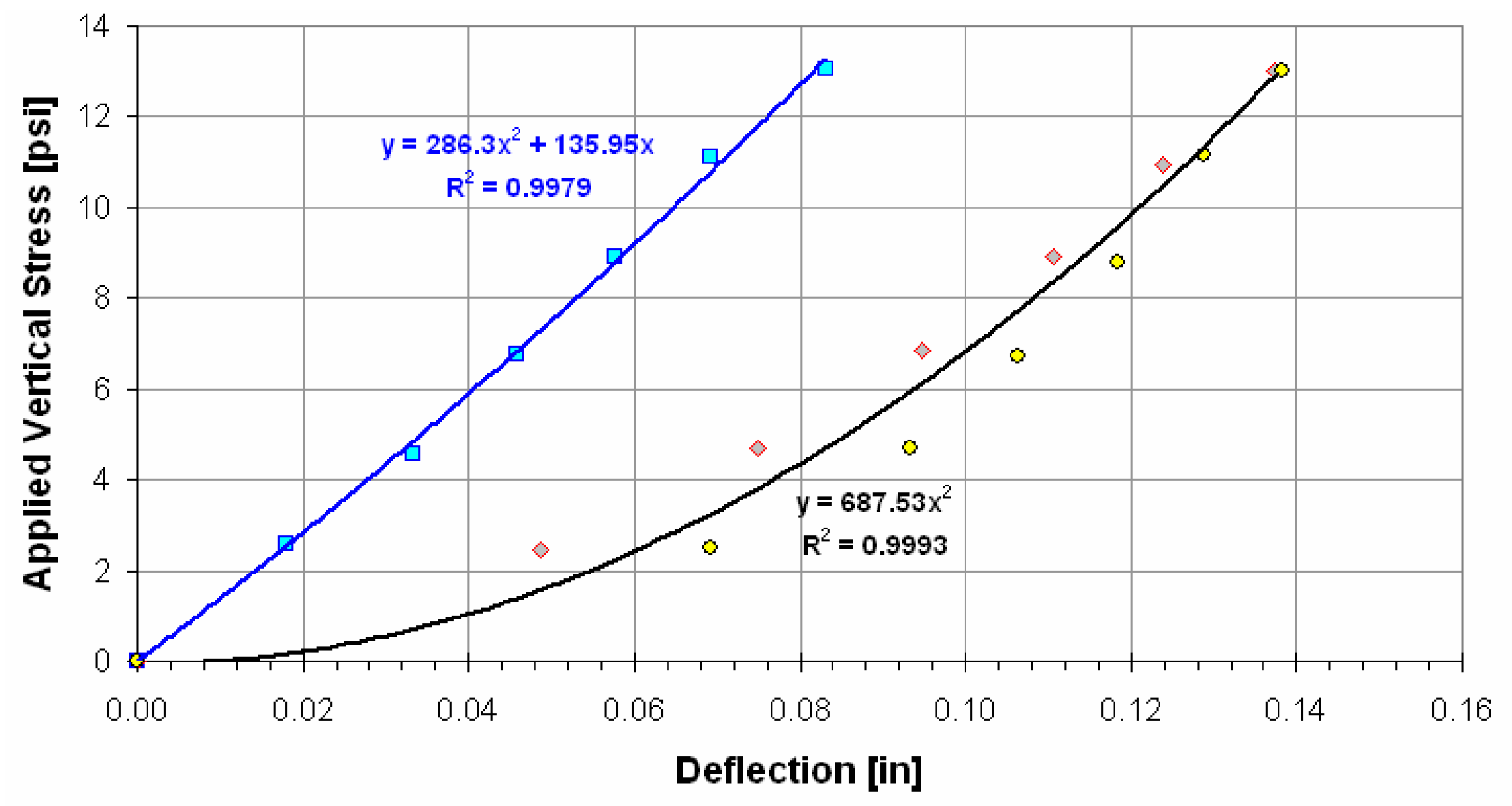

A-2-4 Soil Alone 4

- Recompacted A-2-4 w/Roadrain 3

$\diamond$ A-2-4 Soil w/Roadrain 5

- Poly. (Avg both A-2-4+Roadrain tests)

Figure 5.23: Stress-Deflection Trendlines from Final Trials of All Non-Repetitive Plate Bearing Tests Using A-2-4 Soil Subbases 


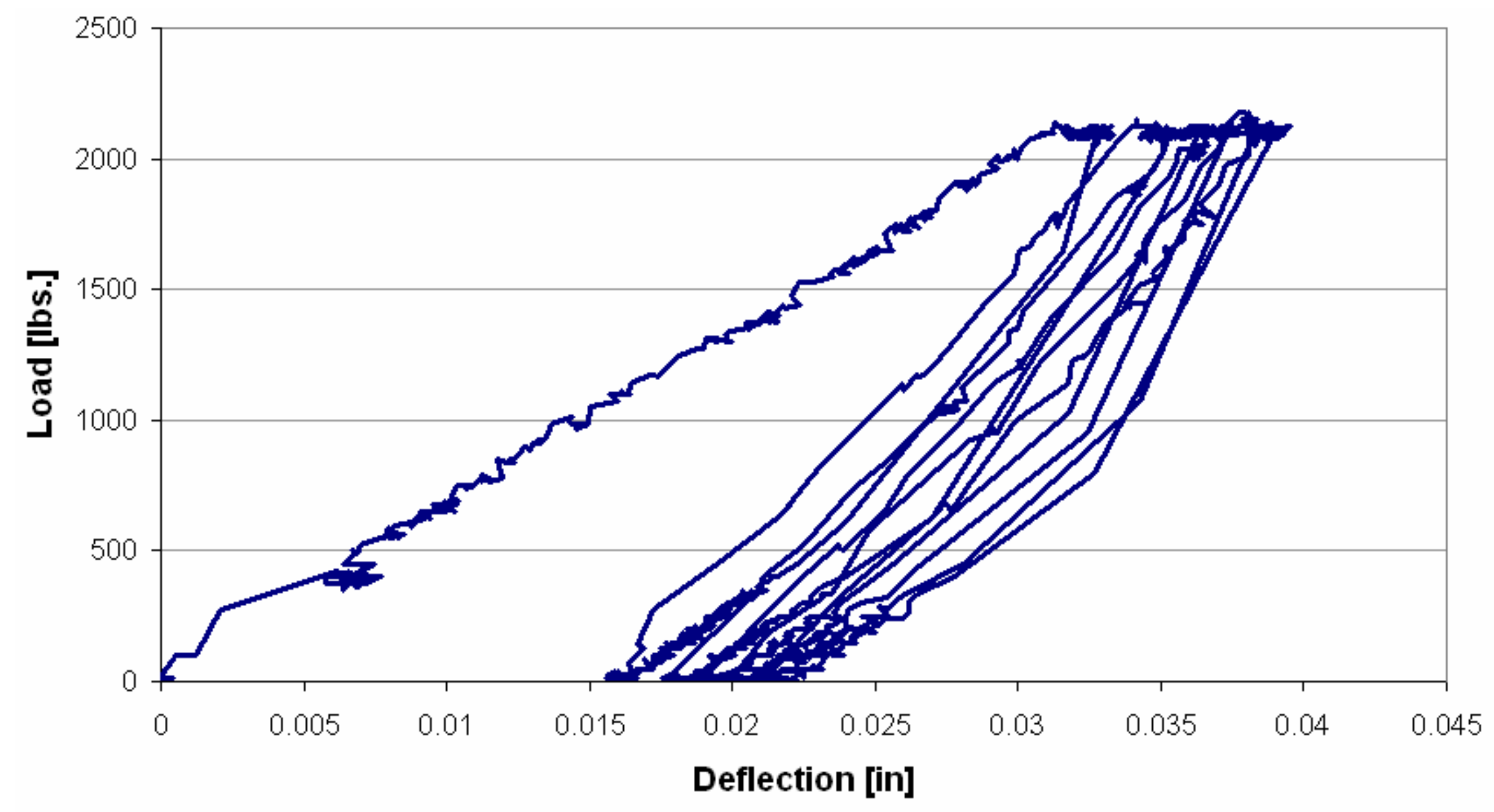

Figure 5.24: Load-Displacement History of \#57 Limestone Aggregate Subbase (Without Roadrain Layer) When Exposed to A Repetitive Static Plate Loading of 2,100 lbs. 




(a)

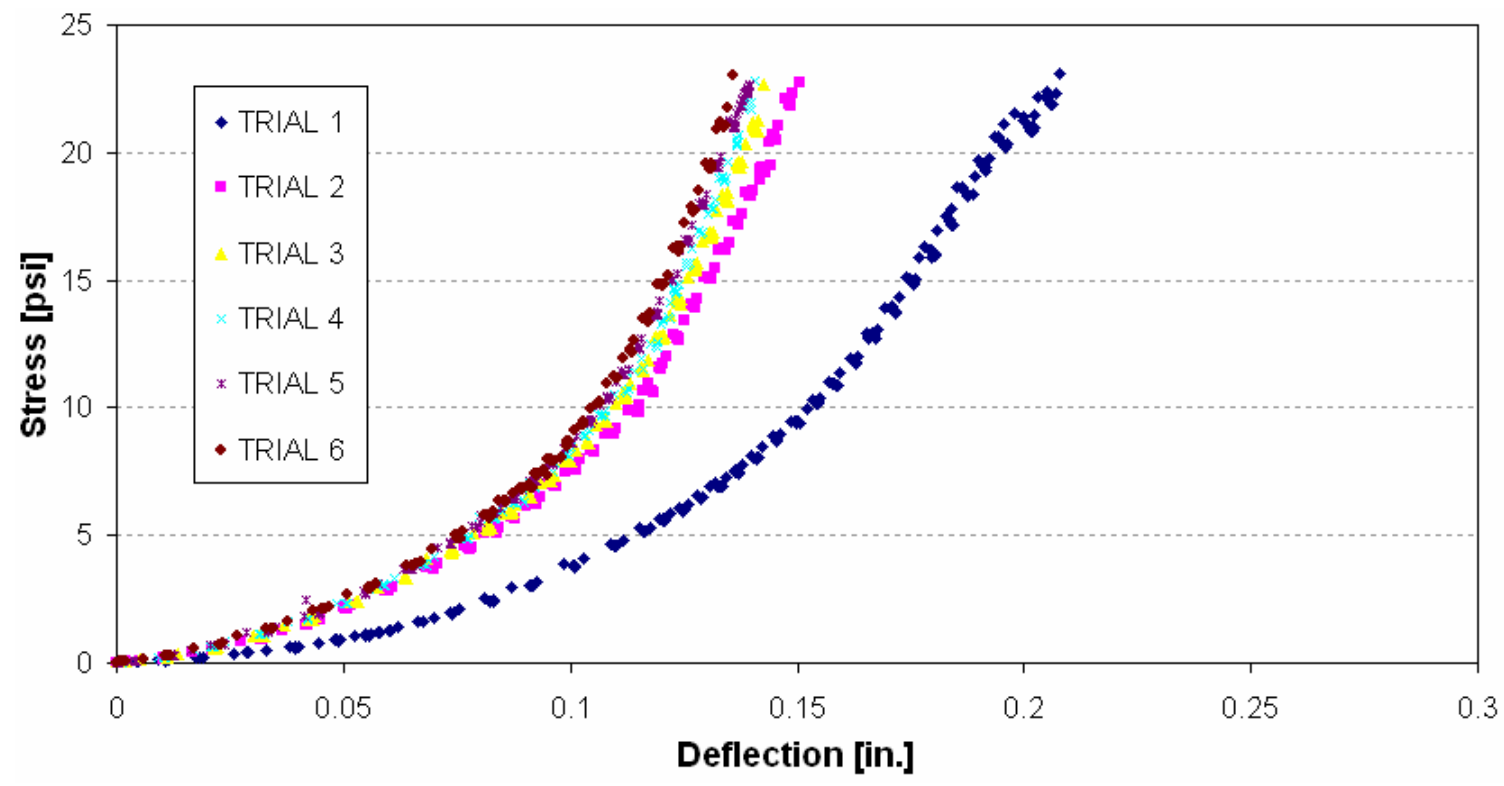

(b)

Figure 5.25: Normalized Stress-Displacement History of an A-2-4 Soil Subbase plus Roadrain Layer When Exposed to a Repetitive Static Plate Loading of 16,000 lbs (a) Initial Curve, and (b) Normalized Curve after Removal of Plastic Deformation. 


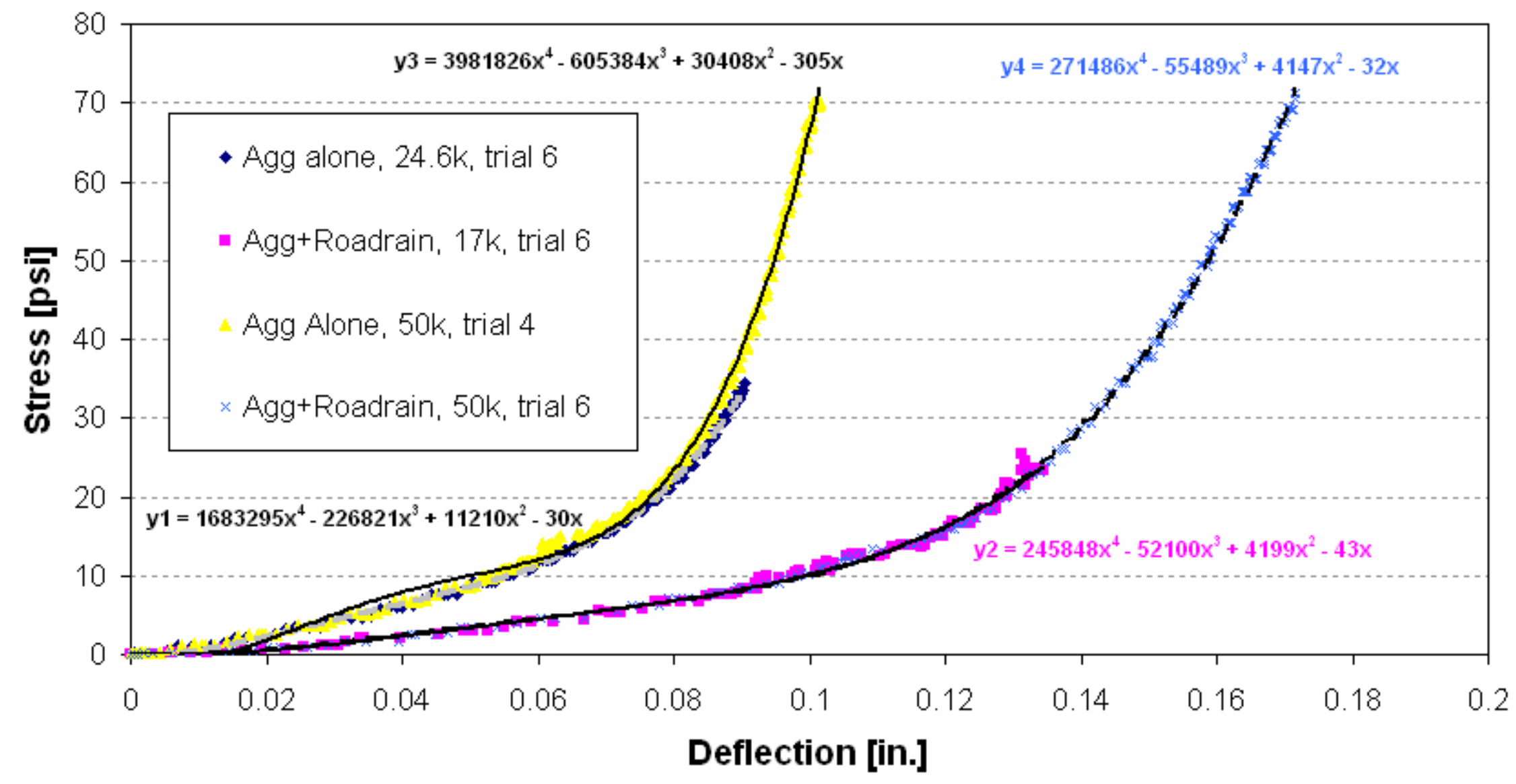

Figure 5.26: Characteristic Curves Obtained from Repetitive Plate Bearing Tests of All Support Systems Using \#57 Limestone Aggregate Subbases Subjected to Multiple "Medium" Load and "Large" Load Applications 


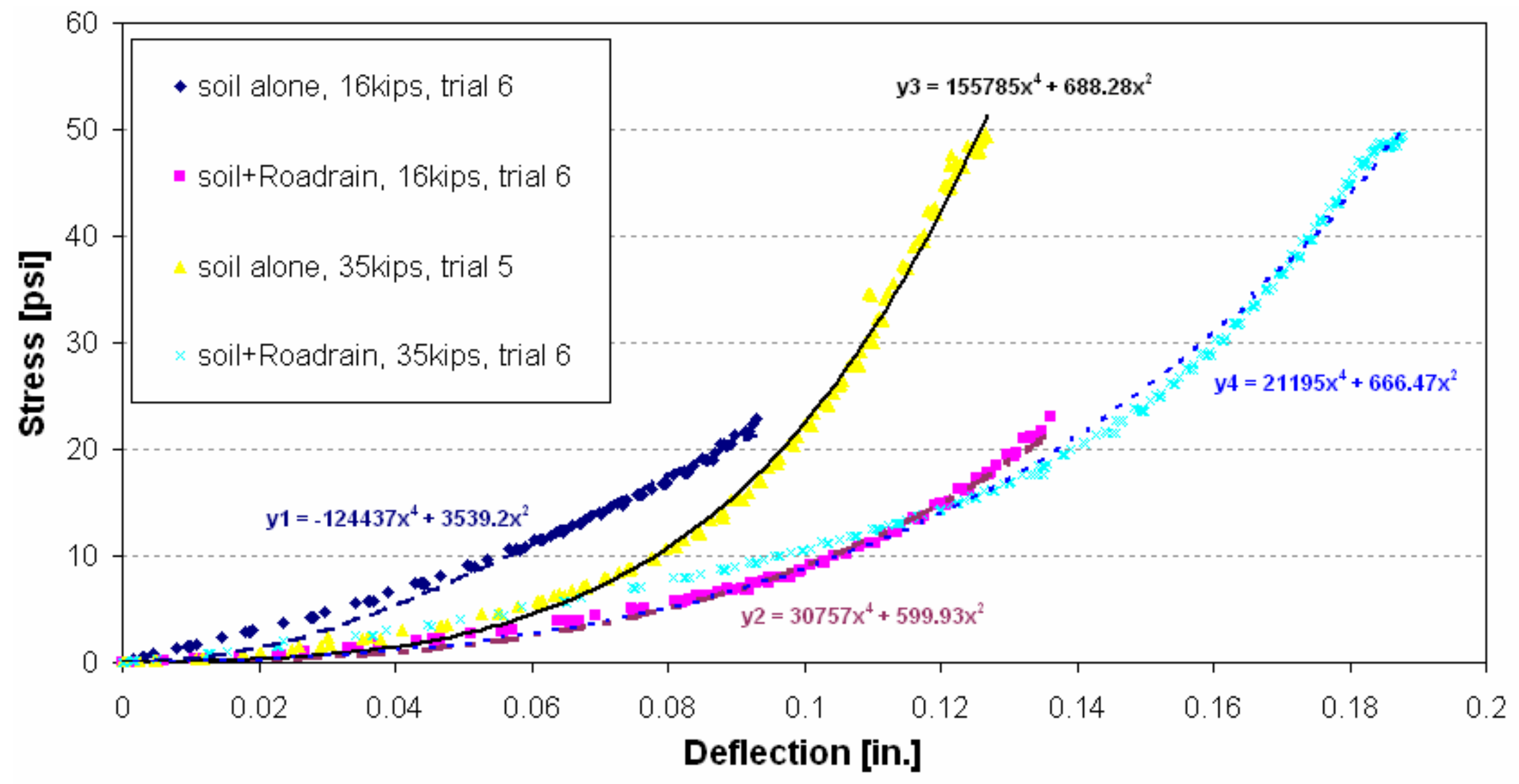

Figure 5.27: Characteristic Curves Obtained from Repetitive Plate Bearing Tests of All Support Systems Using A-2-4 Soil Subbases Subjected to Multiple "Medium" Load and "Large" Load Applications 


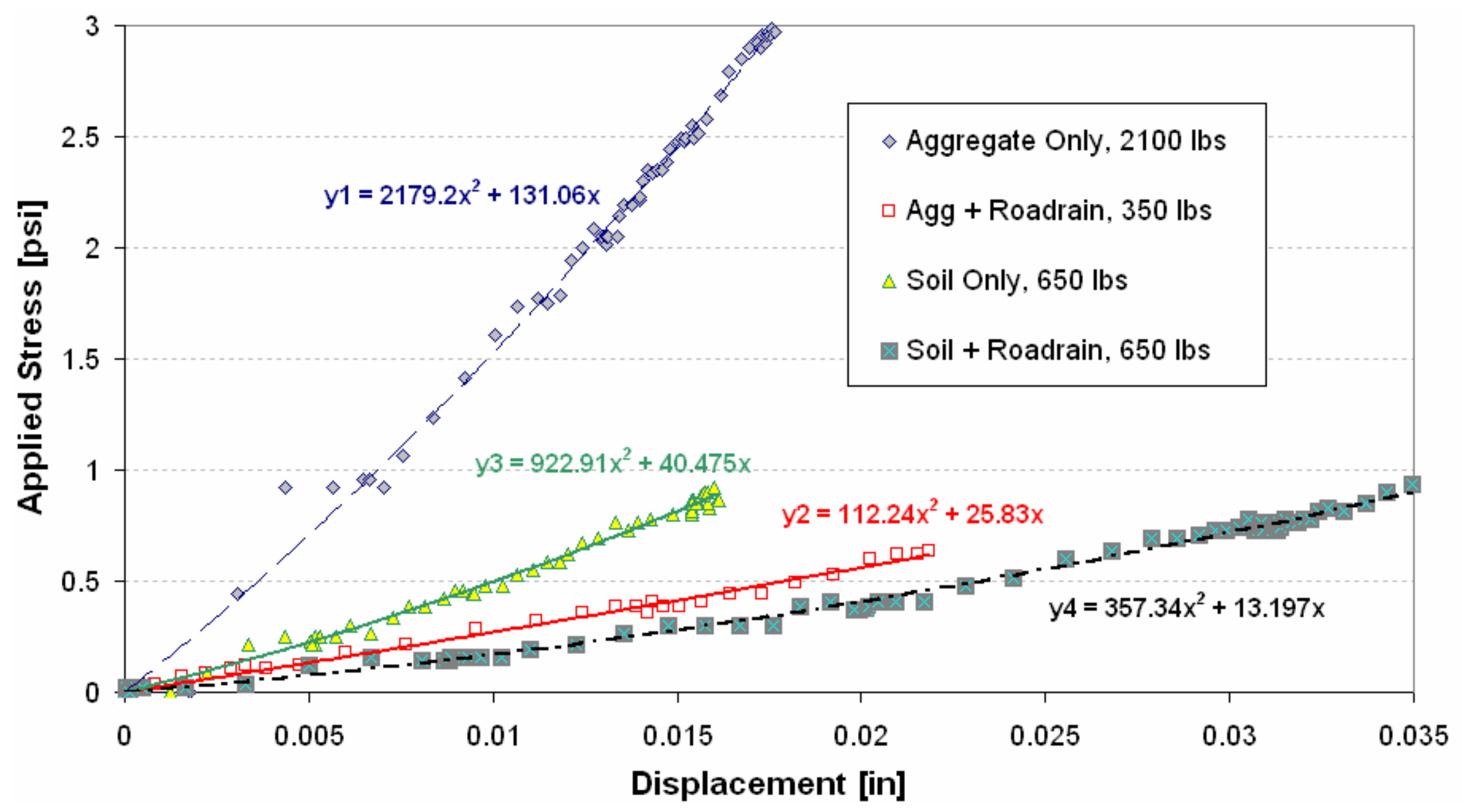

Figure 5.28: Characteristic Curves Obtained from Repetitive Plate Bearing Tests of All Support Systems Subjected to Multiple "Small" Load Applications 




Figure 5.29: Comparison of All Characteristic Plate Bearing Test Loading Curves Obtained with \#57 Limestone Aggregate Subbase 


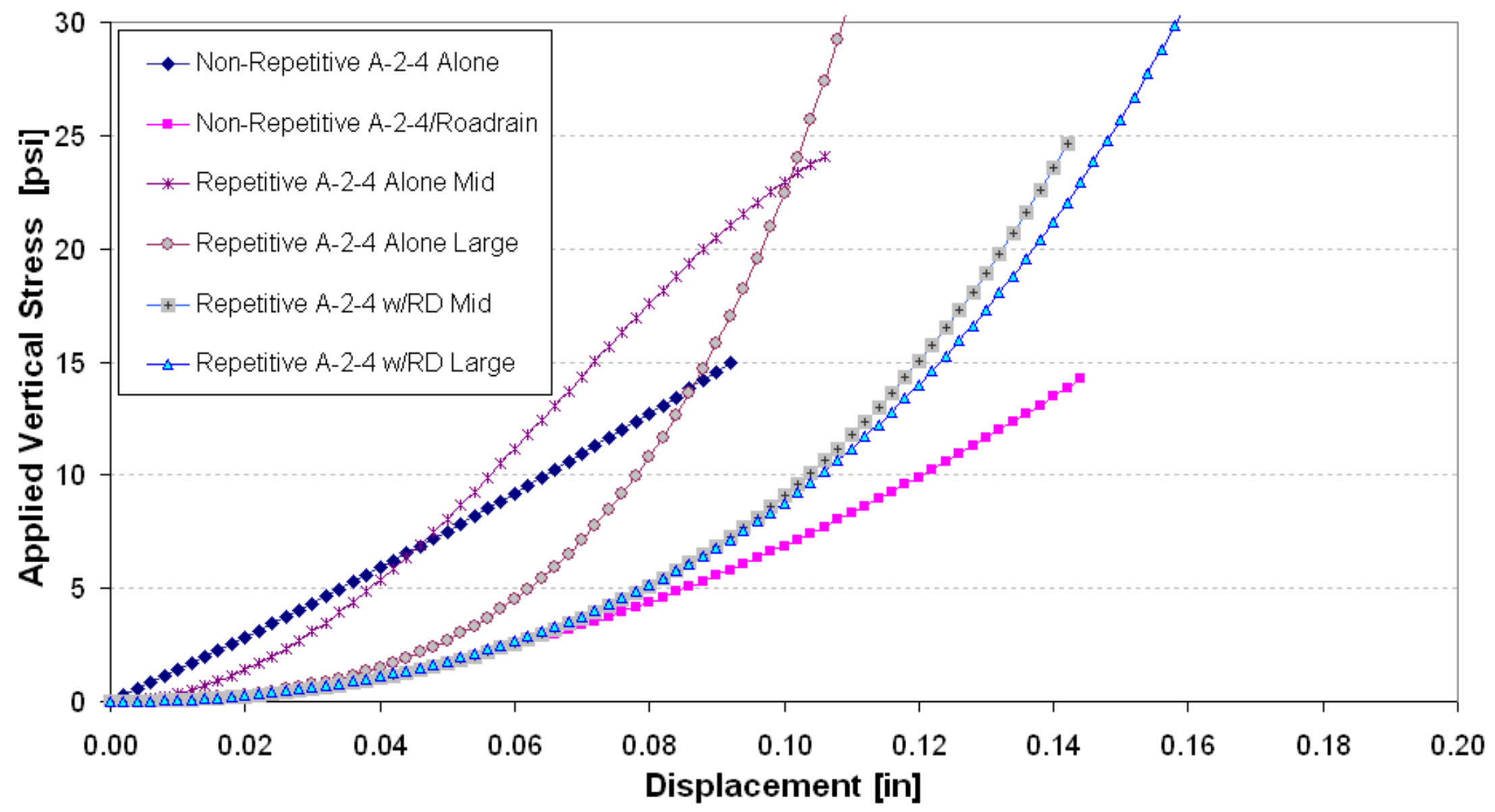

Figure 5.30: Comparison of All Characteristic Plate Bearing Test Loading Curves Obtained with A-2-4 Soil Subbase 




Figure 5.31: Sample of Unloading Curves from the Static Plate Bearing Tests; Obtained from the Non-Repetitive Loading of a \#57 Limestone Aggregate Subbase plus a Roadrain Layer 


\section{CHAPTER 6 \\ PREDICTION OF SUPPORT STIFFNESS}

In order for design engineers to make use of the stiffness characteristics of Roadrain that were determined in the preceding chapters, they must have a means of incorporating this knowledge into an overall pavement design. As mentioned in Chapter 2, both AASHTO and PCA design methods utilize a liquid foundation model, or Winkler foundation, to define the overall support of a PCC pavement provided by the underlying layers. This chapter will briefly propose a simple theoretical design procedure for adjusting the aforementioned support stiffness to a value that can be used by engineers to represent the support when incorporating a Roadrain layer into their pavement system. It will also use the data obtained from the Non-Repetitive Static Plate Bearing Tests discussed in Chapter 5 as a basis of evaluating this analysis technique. FE models are also created for the purpose of determining soil properties from the plate bearing tests and for comparison to the theoretical results.

\subsection{Basic Principle}

For the sake of simplicity of calculation, the addition of the Roadrain layer will be treated as the addition of a one-dimensional spring element, as was the case with the vertical compression tests. Therefore, the deflection of the entire support system under a given loading will be assumed to be equal to what the deflection of the soil layers would be without the addition of the Roadrain layer plus the deflection of the Roadrain layer itself under the given load.

While using a one-dimensional model may not be the standard procedure for incorporating layers into a soil system, as two-dimensional models are typically used, this assumption is not believed to dramatically affect the calculation of the overall system deflection. Two-dimensional models are generally used because the addition of a soil layer would redistribute the load over a larger area, consequently changing the stress distribution at the interface of the underlying layer and ultimately changing its deflection. However, this affect from the addition of a Roadrain layer is believed to be inconsequential for the following reason: the thin layer $(1 / 2$ inch $)$ of Roadrain lacks the 
rigidity in bending and the ability to transfer large amounts of shear necessary to dramatically increase the area on which the applied vertical force is acting. Therefore, the assumptions that the loading area will not be altered, and that the soil stress between layers is the same as at that which would occur directly below the loading plates are assumed to have only nominal affects on elastic settlement calculations.

This idea is supported by visual inspection of the materials after the conclusion of tests involving Roadrain. It could be seen that in addition to the distinct impression of the loading plates on the Roadrain layer the size of the plates, clear impressions only slightly larger than the largest plate were seen both on the bottom of the Roadrain (see Figure 6.1) and in the soil subgrade. This indicates that the area of loading on the soil was approximately the same size both with and without the Roadrain, leading one to believe that the soil layer would experience approximately the same stresses and deflections whether or not the Roadrain was present.

\subsection{General Procedure}

To determine the stiffness of a soil system which uses a Roadrain layer on top, a procedure was devised to theoretically predict the results of a plate bearing test for the system. This was based on the theory discussed in the previous section, and the steps to do so are as follows:

1. Determine the overall $\mathbf{k}$-value of the underlying layers. This can be done experimentally, or by approximating material properties and using one of the methods mentioned in Chapter 2 or another method such as Finite Element Analysis.

2. Using the stiffness from Step 1 , determine the elastic settlement, $S_{e}$, of the soil layers when subjected to a given increase in loading, $\Delta \sigma$. This is simply the range of the applied stress divided by the k-value from Step 1 .

3. For the given stress range, determine a straight-line approximation of the Roadrain's non-linear stress-strain relationship (most likely the slope of a chord going from the points corresponding to the upper and lower limits of the stress to be applied). To predict the results of Non-Repetitive Plate Bearing tests, the results from the sustained loading compression tests would probably be more appropriate due to the similar nature of loading, whereas the load-release compressive test results would be more fitting for the Repetitive Plate Bearing tests.

4. If desired, adjust the stiffness from Step 3 to reflect long-term effects using the results from the cyclic fatigue tests of Chapter 4 . This may include an increase of the modulus and/or a reduction of the thickness of the Roadrain layer. 
5. Using the new Roadrain modulus and thickness, determine the deflection of the Roadrain under the applied load. This would be:

$$
\Delta \mathrm{t}=\left(\mathrm{E} * \mathrm{t}_{\mathrm{o}}\right) / \Delta \sigma
$$

6. Calculate the total settlement for the system, $S_{e, t o t a l}$.

$$
\mathrm{S}_{\mathrm{e}, \text { total }}=\mathrm{S}_{\mathrm{e}}+\Delta \mathrm{t}
$$

7. Calculate the overall system stiffness, $\boldsymbol{k}_{\text {total }}$ :

$$
\mathrm{k}_{\text {total }}=\Delta \sigma / \mathrm{S}_{\mathrm{e}, \text { total }}
$$

\subsection{FE Analyses for Determining Soil Properties}

To ensure that the theoretical model gives the best representation of the overall behaviors seen in the laboratory during the plate bearing tests, the material properties were first determined using FE modeling (FEM). In particular, two FE analyses were done to verify the values used to represent the Young's Moduli, E, and the Poisson's ratios, $v$, for both the \#57 limestone and the A-2-4 soil subbases. These models will be described throughout this section.

\subsubsection{General FEM Characteristics}

Using Abaqus software, axisymmetric models were created to duplicate the loaddeflection data from the Non-Repetitive Static Plate Bearing Tests conducted in the absence of Roadrain. The models for both soil types used the same basic components and outer geometries, which are shown in Figure 6.2. As can be seen, the basic components in each model are the soil subbase, the five A36 steel loading plates, and a high-strength steel piston to distribute the load to the top plate. All components of the models were divided into square, axisymmetric solid elements which had dimensions of 0.5in. $x$ 0.5in..

Boundary conditions were imposed on the bottom and outer edges of the soil subbase components to simulate the boundaries of the actual experimental setup. On the outer edge, displacement was allowed in only the vertical direction and no rotations were permitted; on the bottom, all displacements and rotations were restricted. Material interfaces between components were defined such that all contact surfaces were considered "hard" in the normal direction so no nodal penetration of adjacent surfaces 
was permitted; in the tangential direction, there was assumed to be no frictional resistance to movement between layers.

\subsubsection{Material Properties Used for the Analyses}

In order to properly define the materials for the Abaqus model, the Young's modulus, E, and the Poisson's ratio, $v$, had to be defined for each material. The behavior of the components which were composed of either the A36 structural steel or the highstrength was taken as perfectly linear-elastic in the relatively low range of applied stresses. For the A36 steel used for the plates, the Young's modulus was defined as $29 \times 10^{6} \mathrm{psi}$, and the Poisson's ratio was defined as 0.3 . The exact high-strength steel characteristics were considered to be arbitrary, so long as they allowed for the necessary distribution of force to the top plate; since the metal used for the actual piston is known to be considerably stronger than the A36 structural steel used for the plates, the values used were $60 \times 10^{6} \mathrm{psi}$ and 0.3 for the material's Young's modulus and Poisson's ratio, respectively.

It is known that most soils exhibit non-linear stress-strain relationships (ASCE), as can be seen from the results of the plate bearing tests conducted on the \#57 Limestone and the A-2-4 soil subbases without a Roadrain layer; these results were discussed in Chapter 5, and the non-linearity was illustrated by Figures 5.22 and 5.23. Fortunately, many FEA programs have the capability of analyzing models containing material nonlinearities. However, the exact values of $E$ and $v$ were not known for either of the soil types, since uniaxial or triaxial compression tests were not performed; the nearest thing to a uniaxial or triaxial compression test that was conducted would be the plate bearing tests performed on the soil subbases alone, which included an unknown level of confinement. Therefore, a trial-and-error procedure was followed where the stress-strain relationships of Figures 5.22 and 5.23 were adjusted in the FE model until the deflections obtained were reasonably close to those from the corresponding laboratory experiment. It was desired to keep the same general shapes of the curves, so the adjustments were made simply by multiplying the stress applied from the loading plates by a certain factor in order to increase or reduce the actual average stress level in the soil; meanwhile the Poisson's ratio values were kept reasonably close to the expected values which were 
listed in Table 5.1. This was done until an acceptable combination was obtained which gave displacements comparable to those observed in the laboratory tests.

\subsubsection{Results from Material Property Verification Models}

The deflections of the non-repetitive plate bearing tests were simulated using FE techniques. The plots of the stress-displacement relationships obtained from the laboratory tests of both subbases without Roadrain compared to the results from the corresponding FE simulations, can be seen in Figures 6.3(a) and (b). For the \#57 limestone subbase, the stress was reduced by a factor of 0.85 , altering the stress-strain relationship of Figure 5.22. The corresponding Poisson's ratio used in the FE model for the \#57 limestone subbase was 0.18 . The resulting load-displacement curve obtained for the limestone base is shown in Figure 6.3(a). The stress reduction factor used for the applied stress with the A24 soil subbase was 0.75 , and a Poisson's ratio of 0.23 was used. This gave a good agreement between FE and observed results at each of the load levels, as can be seen in Figure 6.3 (b). The stress-strain relationships and Poisson's ratio values used for all materials in these analyses are shown in Table 6.1.

In addition to the deflections at a location of $1 \mathrm{in}$. from the outside of the top of largest plate, deflections were noted at the center of the top surface of the same plate. These locations are denoted in Figure 6.4 as points A and B, respectively. The differences in deflection Points $\mathrm{A}$ and Point $\mathrm{B}$, $\mathrm{u}_{\mathrm{B}-\mathrm{A}}$, were used to make comparisons between the inner and outer deflections could be made in order to quantify the actual rigidity of the plate system used. The $\mathrm{u}_{\mathrm{B}-\mathrm{A}}$ values increased at the same rate for both $\mathrm{FE}$ analyses as the designated load values increased. Coincidentally, the two subbase types experienced approximately the same differential settlement at each load value, as indicated by the nearly identical values of $\mathrm{u}_{\mathrm{B}-\mathrm{A}}$ for the two soil types; this is not necessarily believed to be the case for all soil types though, as it may vary due to large differences in soil properties.

Since the analyses were axisymmetric, all components were cylindrical in shape, which accurately describes the actual geometries of the plates and piston, but the actual soil bed had a more complex outer geometry than the assumed circular footprint. This was not believed to have a great influence, though, since the stress levels were seen to 
diminish quickly beyond the outer edge of the bottom plate, as can be seen by the sample stress distribution shown in Figure 6.5. Since the actual dimensions extended at least 4 feet from the center of loading, well beyond the primary area of influence from the applied load, small differences in the already minute stress levels due to variations in geometry at the outer perimeter would play very little role in the overall system behavior.

\subsection{Sample Theoretical Calculations}

In order to validate the methodology discussed in Section 6.2, theoretical calculations were made using this technique based upon the Non-Repetitive Static Plate Bearing Tests which incorporated Roadrain that were described in Chapter 5. The results of these were then compared to FE Models and the experimental results obtained from the laboratory tests.

\subsubsection{Theoretical Methodology and Results}

The procedure used for the theoretical calculations was that described in Section 6.2. Egorov's equations given in Section 2.7 were used to calculate the elastic settlement 6-inch-thick soil subbases using the soil properties shown in Table 6.1. This was done for both types of subbase using the equations for both flexible and rigid uniformly-loaded plates; the constants that were used, $\alpha_{1}$ and $\alpha_{2}$, were obtained from Figure 6.6. The stress in the Roadrain layer was assumed to be uniform below the area of loading such that the deflections could easily be obtained. This was done using both the initial and eventual sustained loading equations from Table 3.6 to represent the stress-strain relationships for Roadrain, and an initial thickness of $0.5 \mathrm{in}$. for the layer. The resulting deflections at the center of loading for the \#57 Limestone and A-2-4 soil subbases are shown in Tables $6.3(\mathrm{a})$ and (b), respectively.

From these calculated deflections, the overall stiffness of the support systems could be calculated. Figures 6.7(a) and 6.7(b) show the resulting $k$-values for the $\# 57$ and the A-2-4 systems with and without Roadrain. In these figures, the $k$-values were calculated as if the range of applied stress varied from 0 psi to the indicated value. 


\subsubsection{Comparison of FE and Theoretical Results}

Before the evaluation of the assumptions made with respect to the incorporation of a Roadrain layer with respect to the overall settlement of a support system, the results from the elastic settlements calculated using Egorov's equations were first compared to the FE models of soil alone described in Section 6.3. A comparison of the results is displayed in Figures 6.8(a) and 6.8(b) for settlement below the center of the two subbase types. As is expected, the deflections at the center of the flexible plates are seen to be larger in both cases than those of the rigid plate. From Figure 6.8(b) it is seen that the theoretical rigid plate calculation follows fairly closely to the FEM results for the A-2-4 soil. The results from the \#57 limestone subbase did not follow as closely though, as can be seen from Figure 6.8(a); this is not surprising when considering that the FEM results did not precisely follow the same trend as the experimental results, as was seen in Figure $6.3(\mathrm{a})$.

In addition to the results described above, the FE model for A-2-4 soil subbase described in Section 6.3 was expanded to include a Roadrain layer located directly above the subbase and below the largest plate, as shown in Figure 6.9. The outer dimensions of

all common components remained unchanged, but the square element meshes used for all components were more refined than those without Roadrain. For the piston, plates, and subbase components, the dimensions of the elements used were reduced to 0.25 in. $x 0.25$ in. The Roadrain layer itself had element sizes of 0.1in. x 0.1in.. The same soil properties as those shown in Table 6.1 were used for the analysis. The same stress-strain relationships as those from the theoretical calculations, those shown in Table 3.6 from the Sustained loading Vertical Compression tests, were used to represent the Roadrain stiffness. The Poisson's ratio of the Roadrain is not known for sure, but due to the noncontinuous nature of the geonet core it is believed that lateral deflections would be small in comparison to the vertical deflections, resulting in a very small Poisson's ratio for the material; for these FE analyses, it was assumed to be 0.18 .

Figure 6.10 shows a comparison of the deflections from the FE analysis results to the results from the theoretical calculations when using the initial and eventual Roadrain stiffnesses, respectively. It can be seen that for either Roadrain stress-strain relationship (i.e. initial or eventual), the FE deflections fall between those of the corresponding 
theoretical rigid and flexible plate deflections. This is reasonable, since the actual system is neither perfectly rigid nor a flexible loading area subjected to a uniform load, and indicates a good agreement between FE and theoretical methods for determining this type of elastic settlement.

Also from the FEM results of the models incorporating Roadrain it was seen that significantly less plate bending occurs in the presence of the softer layer. This can be seen when comparing the $\mathrm{u}_{\mathrm{B}-\mathrm{A}}$ values shown in Table 6.4 to those of Table 6.2. It can be seen that in this stress range, the presence of the Roadrain layer reduces differential settlement by about $20 \%$ to $25 \%$ from that without the Roadrain under the same applied loading.

\subsubsection{Comparison of Theoretical and Experimental Results}

In order to compare the theoretical settlements, which occur at the center of the bottom plate, to the experimental results, which were measured near the outer edge of the same plate, adjustments were first made to the observed experimental deflections. In the cases of the soil subbases alone, the values from Table 6.2 for $\mathrm{R}_{\mathrm{B}-\mathrm{A}}$ that were obtained from the FE analyses described in Section 6.3 were simply added to the outer deflections to approximate those at the center of the plate. In the cases where Roadrain was used, a similar procedure was done using the values from Table 6.4 for $\mathrm{u}_{\mathrm{B}-\mathrm{A}}$. However, since no FE Analyses using \#57 soil subbases with Roadrain were done, the values for the A-2-4 soil subbases were added to approximate the center deflections for both types of soil.

The deflections from the soil subbases alone, both theoretical and observed, as well as those of the subbases plus a Roadrain layer, are plotted in Figures 6.11 and 6.12 for the \#57 Limestone and the A-2-4 soil subbases, respectively. The deflections calculated for both of the subbases alone were reasonably close to the experimental results. The inclusion of the Roadrain layer did cause some discrepancies between the results, though. Upon inspecting these two figures, it would appear that the theoretical calculations did not properly account for the additional deflection resulting from the addition of Roadrain, since the calculated deflections are significantly lower than the experimental results. This is even the case when reducing the Roadrain stiffness to that 
which was derived from the initial sustained-loading vertical compression tests which were supposed to represent the low stiffness of Roadrain the first time ever being loaded.

The explanation for the bulk of this discrepancy can be explained when considering the experimental setup for the plate bearing tests. For these tests, the section of Roadrain was placed directly below the largest plate; for the vertical compression tests from which the Roadrain properties were derived, though, the Roadrain was bonded on one side to concrete, allowing cement paste to penetrate into the fabric, as would be the case in the field if this were placed directly beneath the PCC pavement. The presence of cement paste in the geocomposite would certainly increase the stiffness of the weaker fabric layers, thereby increasing the overall material stiffness. Consequently, smaller deflections would occur than would be the case of a fresh sheet of Roadrain which had not been exposed to the curing cement paste. Therefore one would expect the weaker Roadrain layer that was used for the experiment to undergo larger deflections than the layer used for the theoretical calculations. The degree to which this affects the resulting deflection would have to be investigated further. 
Table 6.1: Final Material Properties Used in the FE Analyses Representing the Non-Repetitive Static Plate Loadings of Subbases without Roadrain

\begin{tabular}{|c|c|c|}
\hline Component & Stress-Strain Relationship & Poisson's Ratio \\
\hline Piston & $\sigma[\mathrm{psi}]=\left(60 \times 10^{6}\right) \varepsilon$ & 0.3 \\
\hline Plates & $\sigma[\mathrm{psi}]=\left(29 \times 10^{6}\right) \varepsilon$ & 0.3 \\
\hline \#57 Limestone Subbase & $\sigma[\mathrm{psi}]=\mathbf{8 9 , 9 9 2} \varepsilon^{2}+776.2 \varepsilon$ & $\mathbf{0 . 1 8}$ \\
\hline A-2-4 Soil Subbase & $\sigma$ [psi] $=13,742 \varepsilon^{2}+1,087.6 \varepsilon$ & 0.23 \\
\hline
\end{tabular}

Table 6.2: Comparison of Central and Outer Deflections as Obtained from the FE Analyses of Subbases without Roadrain

\begin{tabular}{|c|c|c|}
\hline $\begin{array}{c}\text { Applied Load } \\
\text { [lbs] }\end{array}$ & $\begin{array}{c}\text { \#57 Limestone S.B. } \\
\mathbf{u}_{\mathrm{B}-\mathrm{A}}\end{array}$ & $\begin{array}{c}\text { A-2-4 Soil S.B. } \\
\mathbf{u}_{\mathrm{B}-\mathrm{A}}\end{array}$ \\
\hline $\mathbf{2 5 0}$ & 0.00025 & 0.00025 \\
\hline $\mathbf{1 7 5 0}$ & 0.00167 & 0.00172 \\
\hline $\mathbf{3 2 5 0}$ & 0.00320 & 0.00320 \\
\hline $\mathbf{4 7 5 0}$ & 0.00467 & 0.00468 \\
\hline $\mathbf{6 2 5 0}$ & 0.00612 & 0.00614 \\
\hline $\mathbf{7 7 5 0}$ & 0.00758 & 0.00761 \\
\hline $\mathbf{9 2 5 0}$ & 0.00905 & 0.00907 \\
\hline
\end{tabular}


Table 6.3: Calculated Elastic Settlement [in Inches] at Center of Loading Plate for Subbases Systems with and without Roadrain: (a) \#57 Limestone Subbase, and

(b) A-2-4 Soil Subbase

\begin{tabular}{|c|c|c|c|c|c|c|}
\hline \multirow{2}{*}{$\begin{array}{l}\text { Applied } \\
\text { Load [lbs] }\end{array}$} & \multicolumn{2}{|c|}{$\begin{array}{l}\text { \#57 Limestone } \\
\text { Subbase Alone }\end{array}$} & \multicolumn{4}{|c|}{$\begin{array}{l}\text { \#57 Limestone Subbase plus Roadrain } \\
\left.\text { (Roadrain: } \sigma=2,800 \varepsilon^{2}\right) \mid\left(\text { Roadrain: } \sigma=10,000 \varepsilon^{2}\right)\end{array}$} \\
\hline & Flexible Plate & Rigid Plate & Flexible Plate & Rigid Plate & Flexible Plate & Rigid Plate \\
\hline 250 & 0.00362 & 0.00318 & 0.00924 & 0.008797 & 0.006591 & 0.006150 \\
\hline 1750 & 0.02020 & 0.01774 & 0.03506 & 0.032603 & 0.028063 & 0.025601 \\
\hline 3250 & 0.03258 & 0.02861 & 0.05284 & 0.048870 & 0.043298 & 0.039328 \\
\hline 4750 & 0.04291 & 0.03768 & 0.06741 & 0.062176 & 0.055869 & 0.050640 \\
\hline 6250 & 0.05196 & 0.04563 & 0.08006 & 0.073727 & 0.066827 & 0.060494 \\
\hline 7750 & 0.06012 & 0.05279 & 0.09141 & 0.084079 & 0.076670 & 0.069344 \\
\hline 9250 & 0.06760 & 0.05936 & 0.10178 & 0.093543 & 0.085683 & 0.077445 \\
\hline
\end{tabular}

(a)

\begin{tabular}{|c|c|c|c|c|c|c|}
\hline \multirow{2}{*}{$\begin{array}{c}\text { Applied } \\
\text { Load [lbs] }\end{array}$} & \multicolumn{2}{|c|}{$\begin{array}{c}\text { A-2-4 Soil } \\
\text { Subbase Alone }\end{array}$} & \multicolumn{3}{c|}{ A-2-4 Soil Subbase plus Roadrain } \\
(Roadrain: $\sigma=2,800 \varepsilon^{2}$ ) & \multicolumn{2}{c|}{ (Roadrain: $\sigma=10,000 \varepsilon^{2}$ ) } \\
& Flexible Plate & Rigid Plate & Flexible Plate & Rigid Plate & Flexible Plate & Rigid Plate \\
\hline $\mathbf{2 5 0}$ & 0.00362 & 0.00318 & 0.00924 & 0.008797 & 0.006591 & 0.006150 \\
\hline $\mathbf{1 7 5 0}$ & 0.02020 & 0.01774 & 0.03506 & 0.032603 & 0.028063 & 0.025601 \\
\hline $\mathbf{3 2 5 0}$ & 0.03258 & 0.02861 & 0.05284 & 0.048870 & 0.043298 & 0.039328 \\
\hline $\mathbf{4 7 5 0}$ & 0.04291 & 0.03768 & 0.06741 & 0.062176 & 0.055869 & 0.050640 \\
\hline $\mathbf{6 2 5 0}$ & 0.05196 & 0.04563 & 0.08006 & 0.073727 & 0.066827 & 0.060494 \\
\hline $\mathbf{7 7 5 0}$ & 0.06012 & 0.05279 & 0.09141 & 0.084079 & 0.076670 & 0.069344 \\
\hline $\mathbf{9 2 5 0}$ & 0.06760 & 0.05936 & 0.10178 & 0.093543 & 0.085683 & 0.077445 \\
\hline
\end{tabular}

(b) 
Table 6.4: Comparison of Central and Outer Deflections as Obtained from the FE Analyses of A-2-4 Soil Subbases with Roadrain

\begin{tabular}{|c|c|c|}
\hline $\begin{array}{c}\text { Applied Load } \\
\text { [lbs] }\end{array}$ & \multicolumn{2}{|c|}{$\mathbf{u}_{\mathrm{B}-\mathrm{A}}$} \\
\hline $\mathbf{2 5 0}$ & 0.0002 & 0.0002 \\
\hline $\mathbf{1 7 5 0}$ & 0.0013 & 0.0013 \\
\hline $\mathbf{3 2 5 0}$ & 0.0025 & 0.0024 \\
\hline $\mathbf{4 7 5 0}$ & 0.0036 & 0.0037 \\
\hline $\mathbf{6 2 5 0}$ & 0.0048 & 0.0048 \\
\hline $\mathbf{7 7 5 0}$ & 0.0060 & 0.0060 \\
\hline $\mathbf{9 2 5 0}$ & 0.0073 & 0.0072 \\
\hline
\end{tabular}




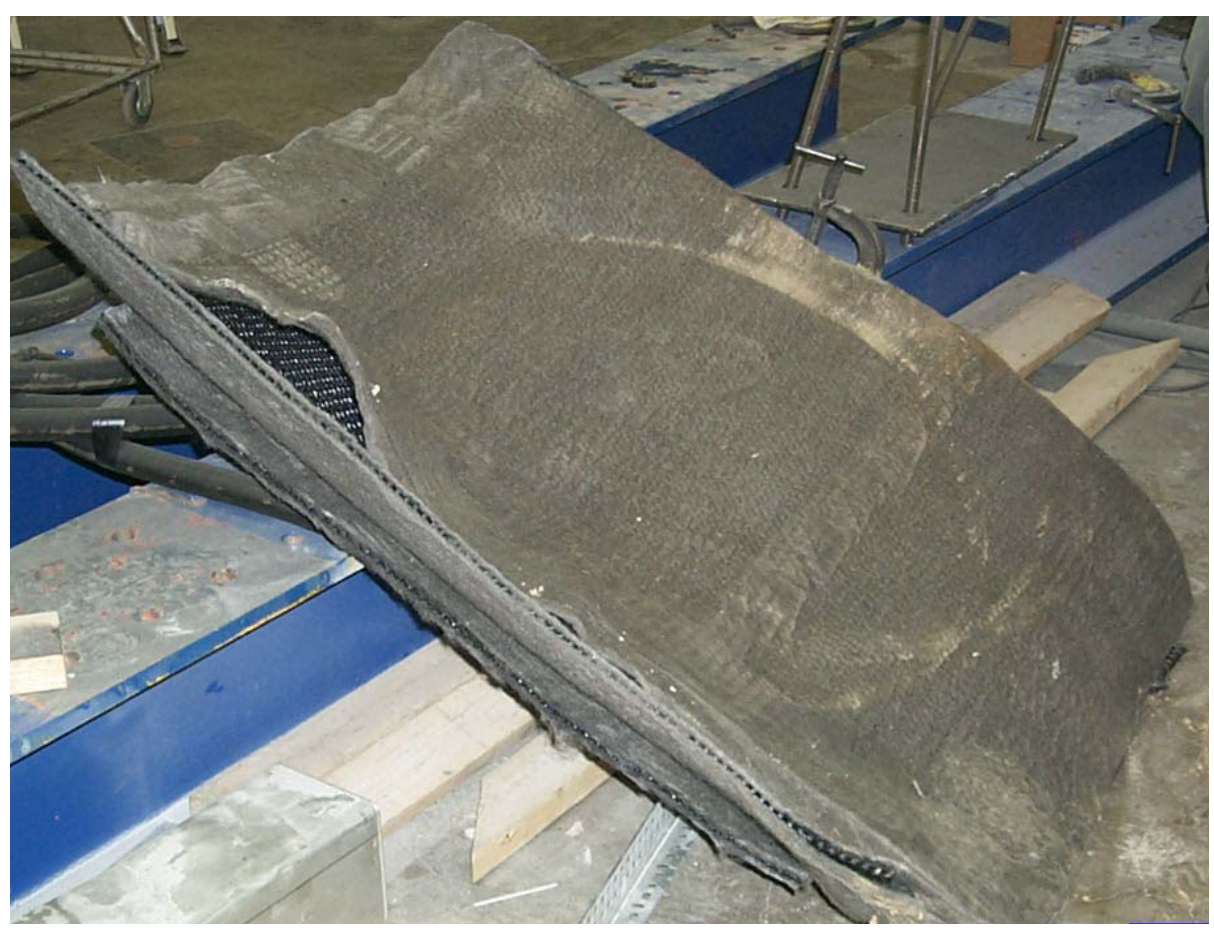

Figure 6.1: Picture of Distinct Imprint on Bottom Side of Roadrain Layer at the Conclusion of a Plate Bearing Test with A-2-4 Soil Subbase

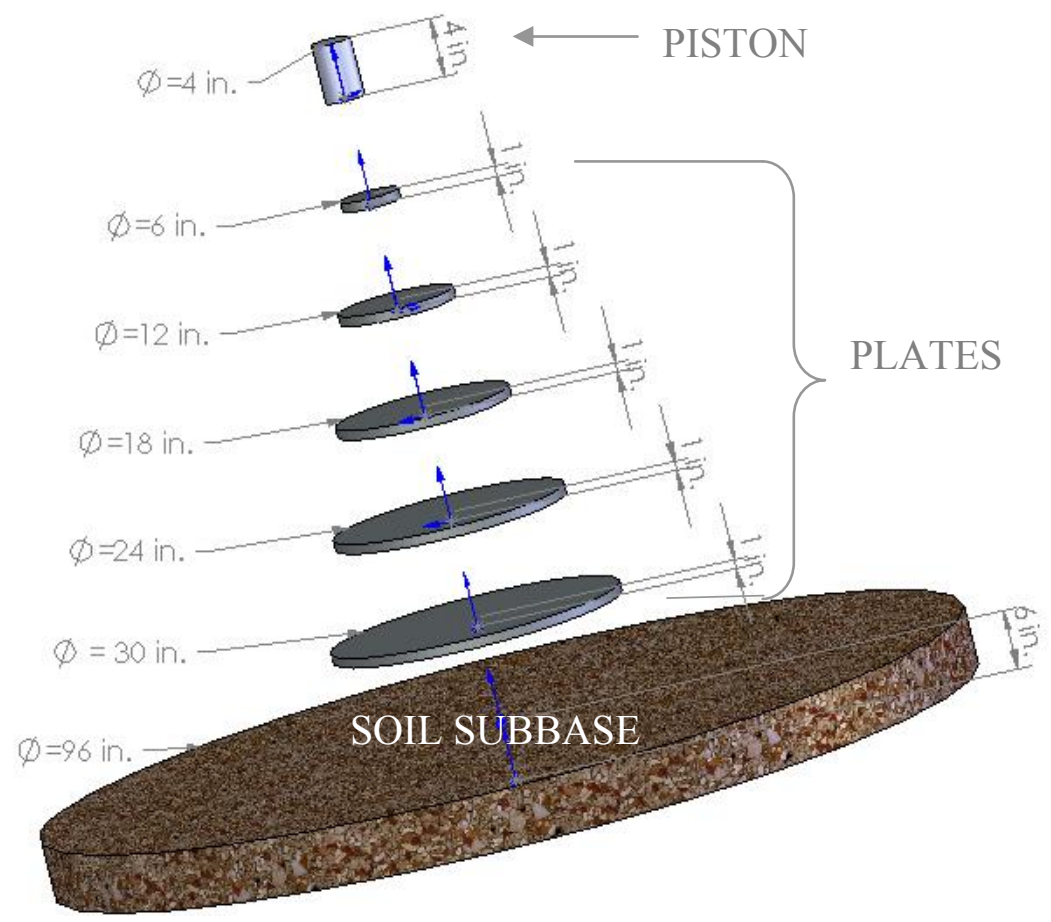

Figure 6.2: Exploded View of Component Geometries used for Axisymmetric FE Modeling of Non-Repetitive Plate Bearing Tests of Soil Subbases without Roadrain 


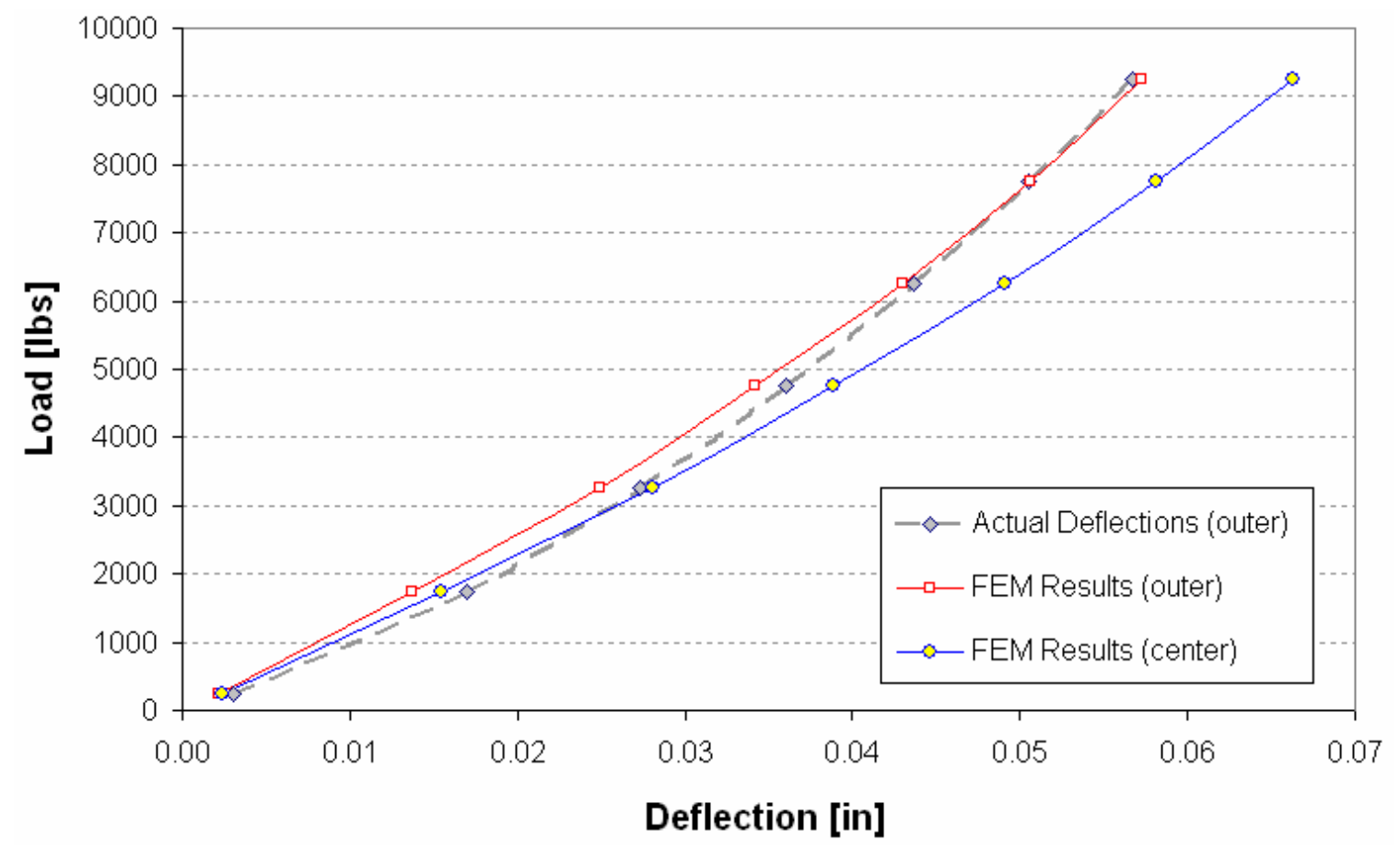

(a)

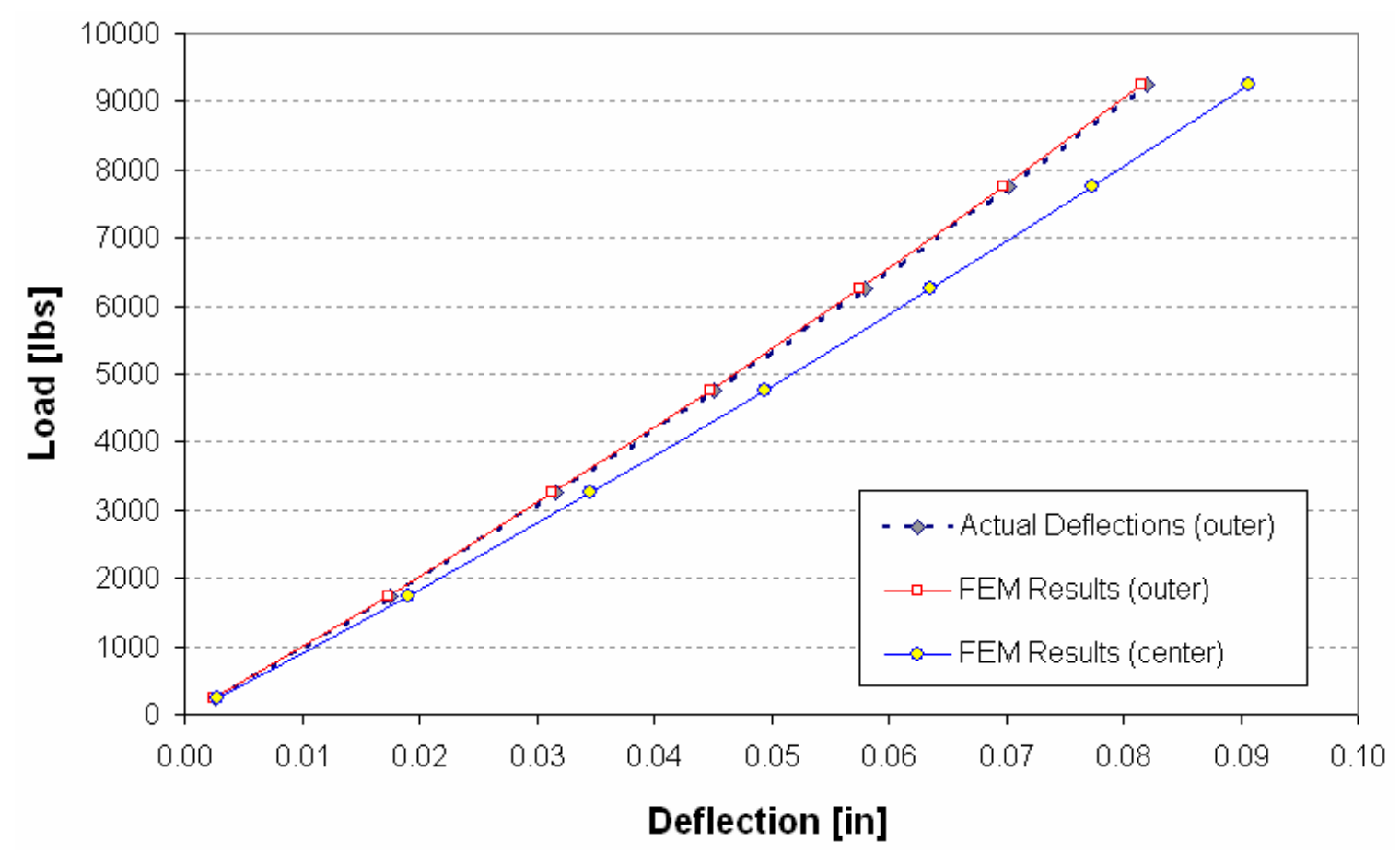

(b)

Figure 6.3: Comparisons of FEA Results to Experimental Data from Non-Repetitive Plate Bearing Tests of Soil Subbases without Roadrain. (a) \#57 Limestone Subbase, and (b) A-2-4 Soil Subbase. 


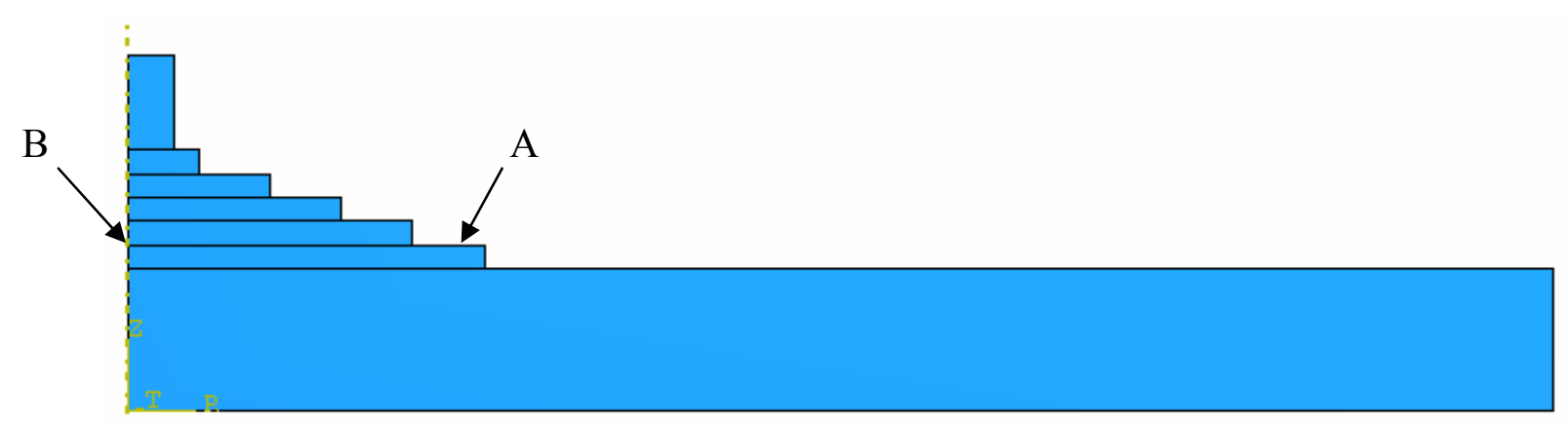

Figure 6.4: Locations of Deflection Readings for FE Analysis of Plate Bearing Tests
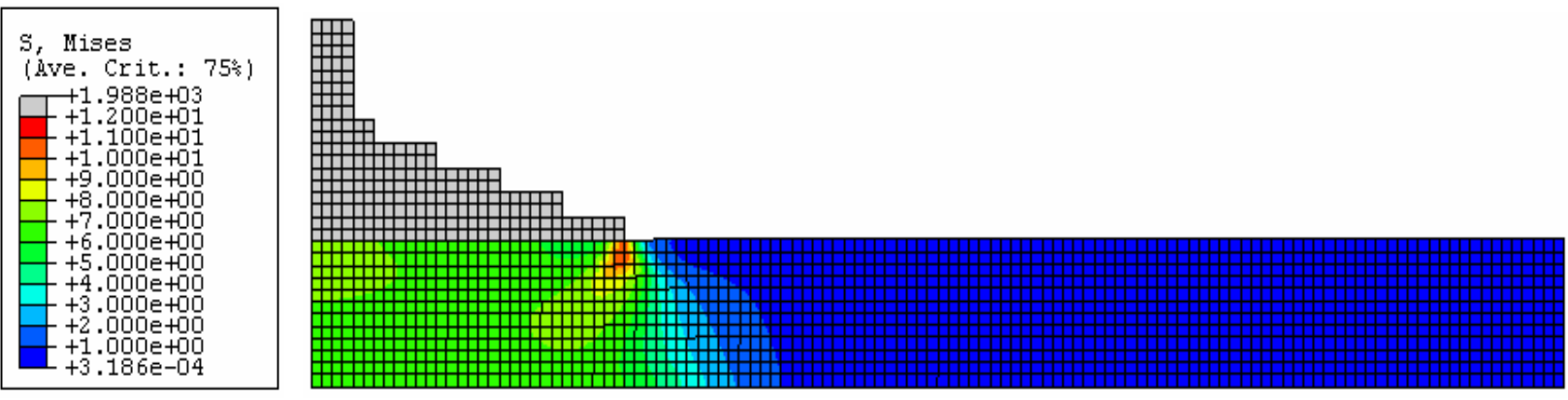

Figure 6.5: Sample Stress Distribution: Load=92501bs. on A-2-4 Soil Subbase Alone

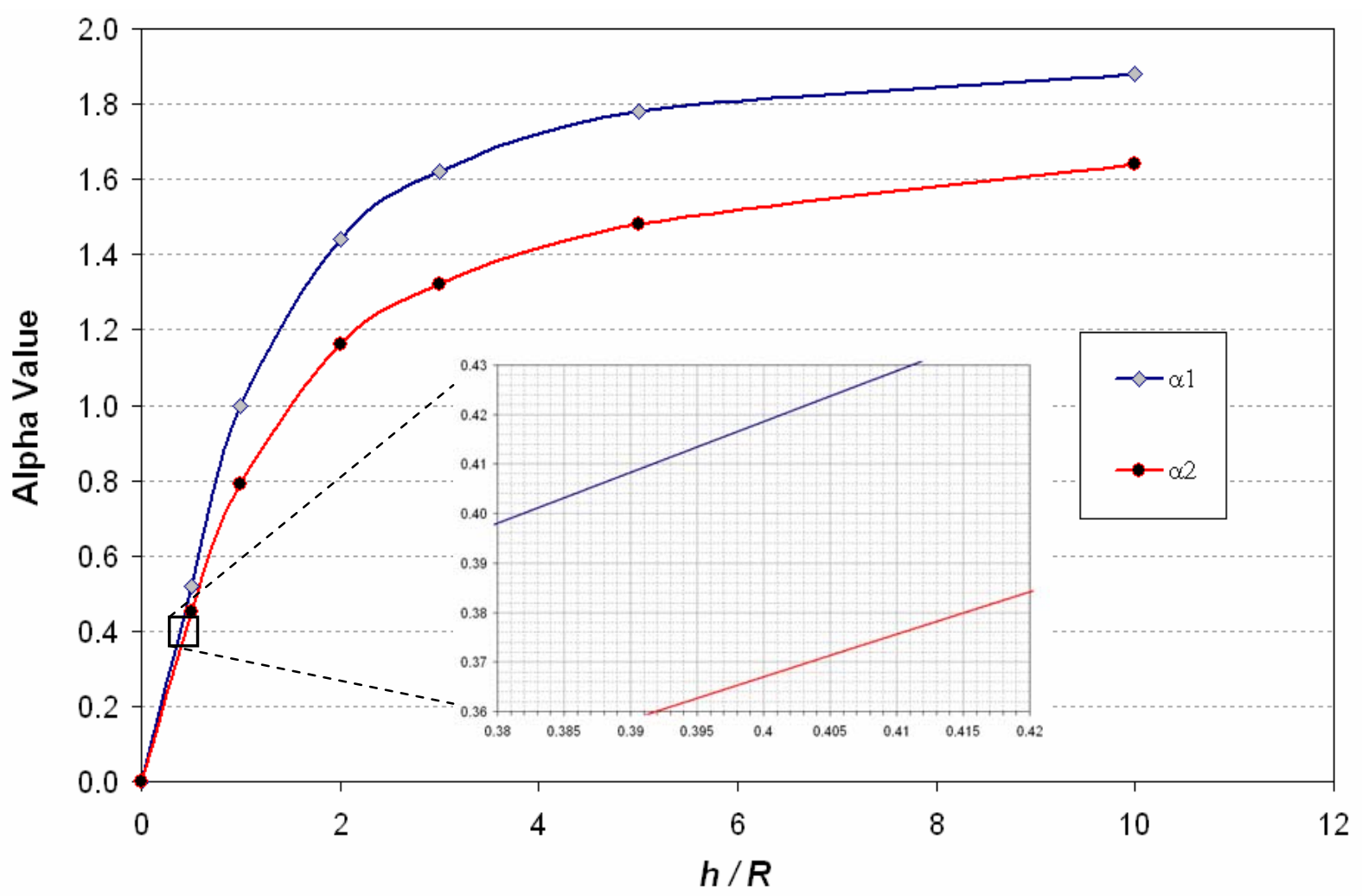

Figure 6.6: Egorov's Constants Used for Theoretical Calculations of Elastic Settlement 


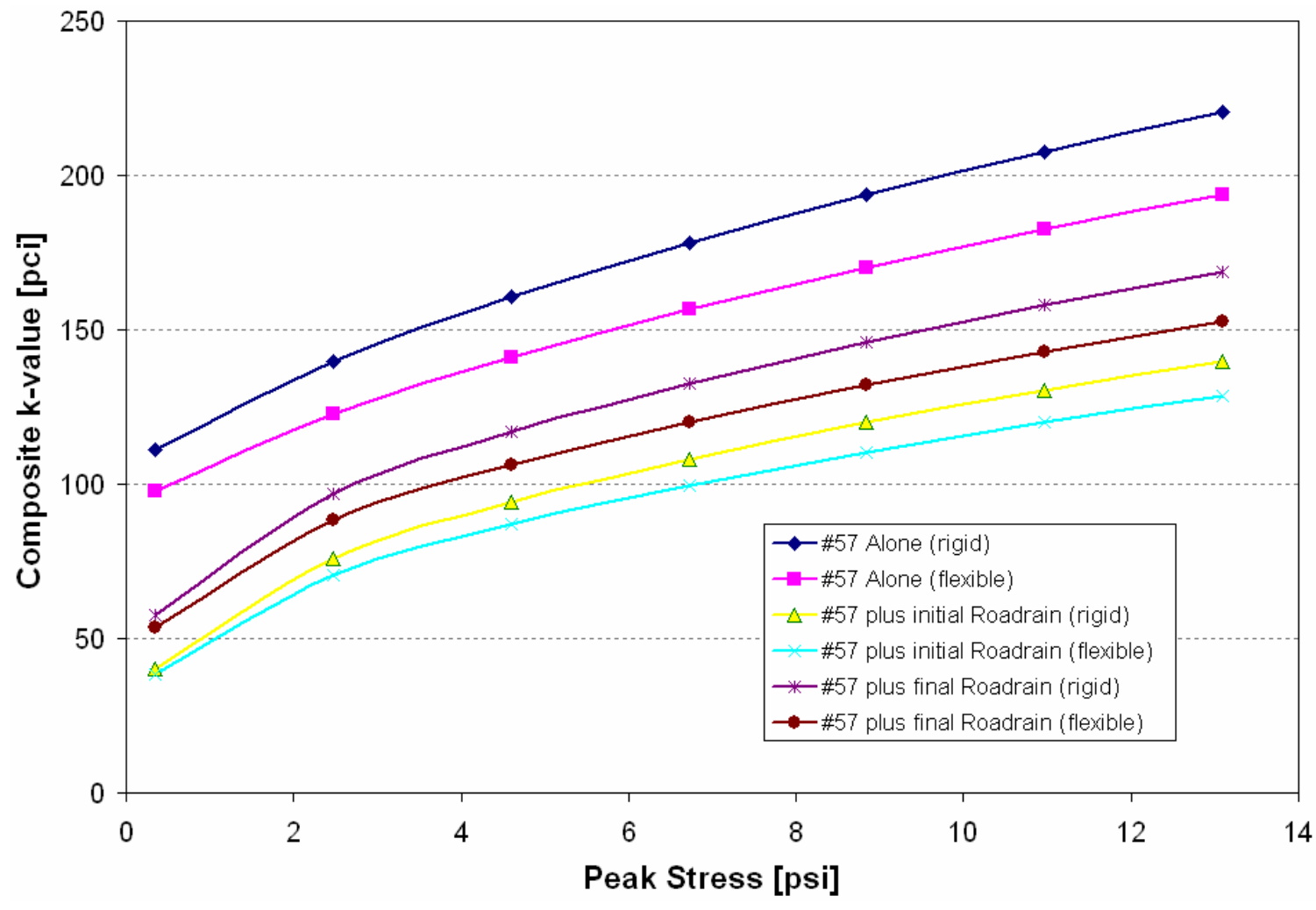

(a)

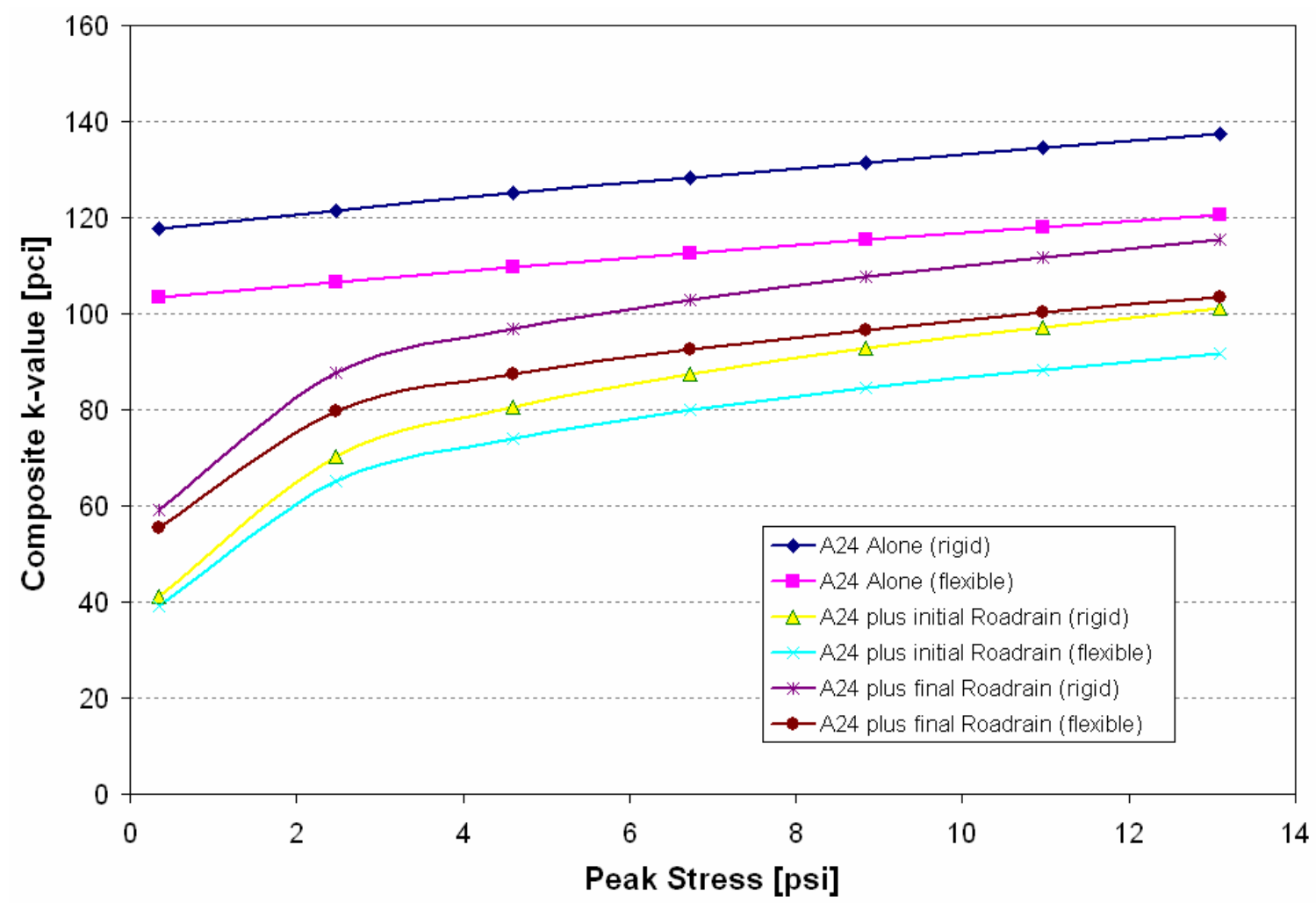

(b)

Figure 6.7: Calculated Theoretical Composite Stiffnesses Assuming Load Range from 0psi to Peak as Indicated. (a) \#57 Limestone and (b) A-2-4 Soil Subbases. 


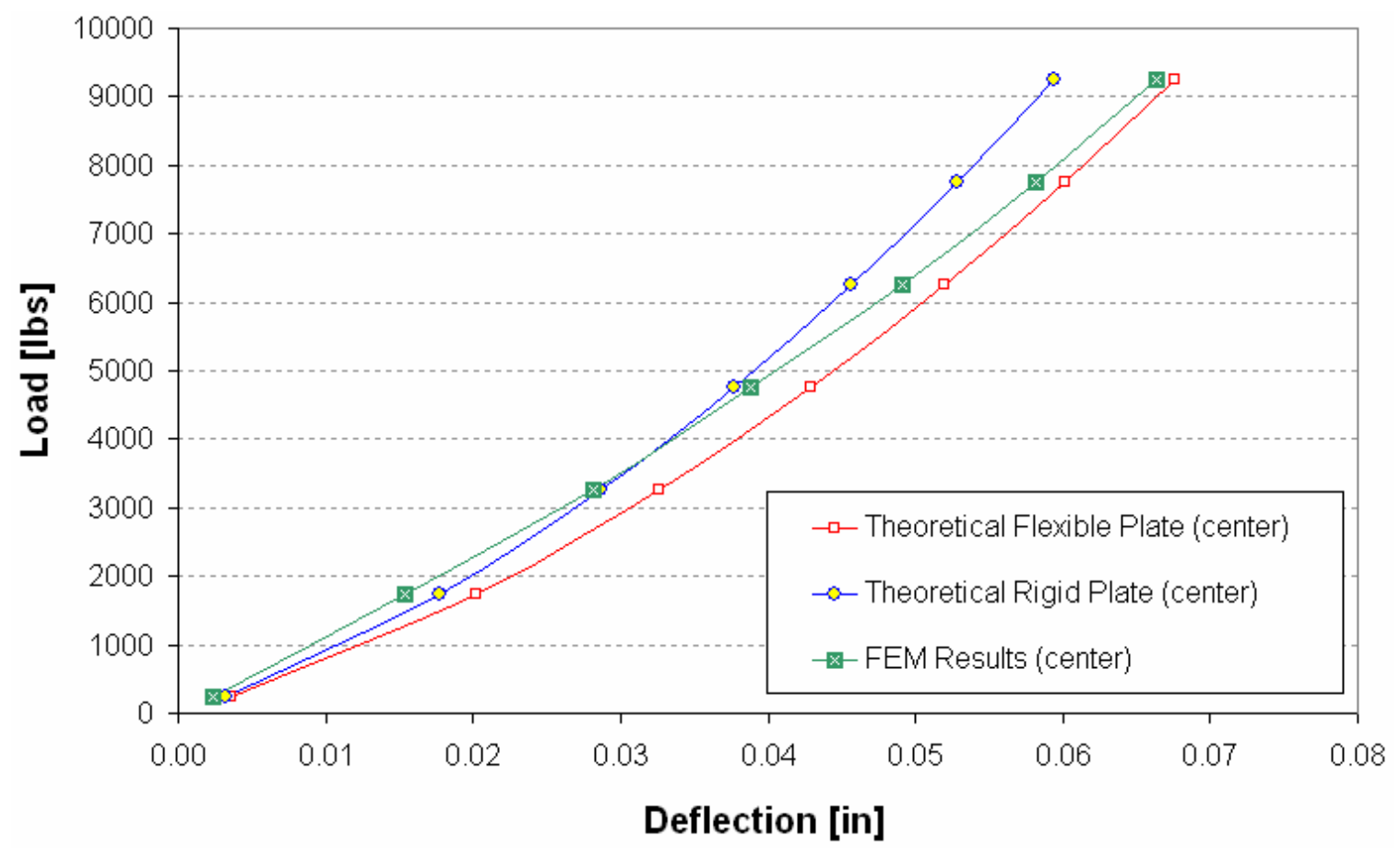

(a)

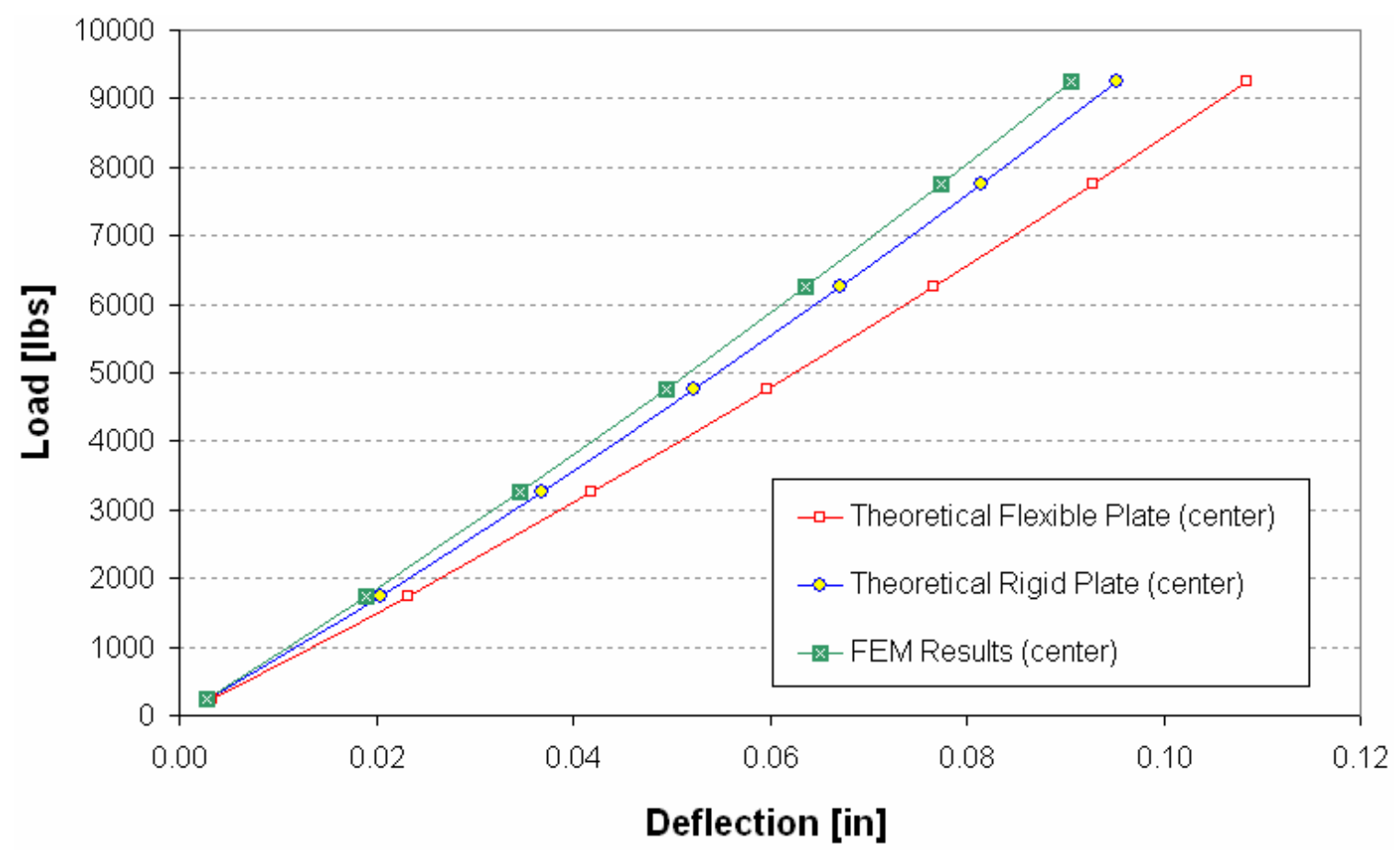

(b)

Figure 6.8: Comparisons of Theoretical Deflections to FEA Results from Simulation of Non-Repetitive Plate Bearing Tests of Soil Subbases without Roadrain. (a) \#57 Limestone Subbase, and (b) A-2-4 Soil Subbase. 


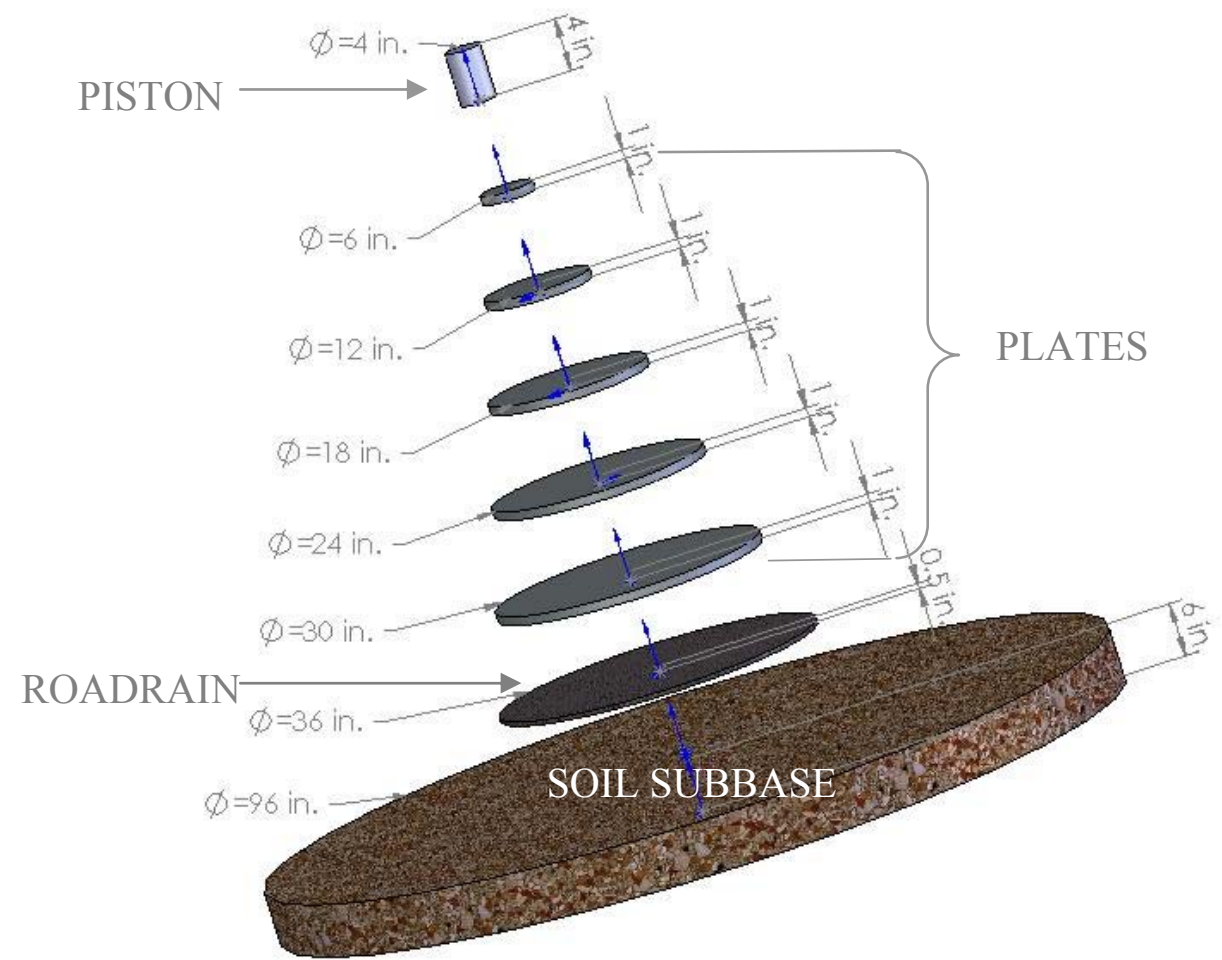

Figure 6.9: Exploded View of Component Geometries used for Axisymmetric FE Modeling of Non-Repetitive Plate Bearing Tests of A-2-4 Soil Subbase with Roadrain

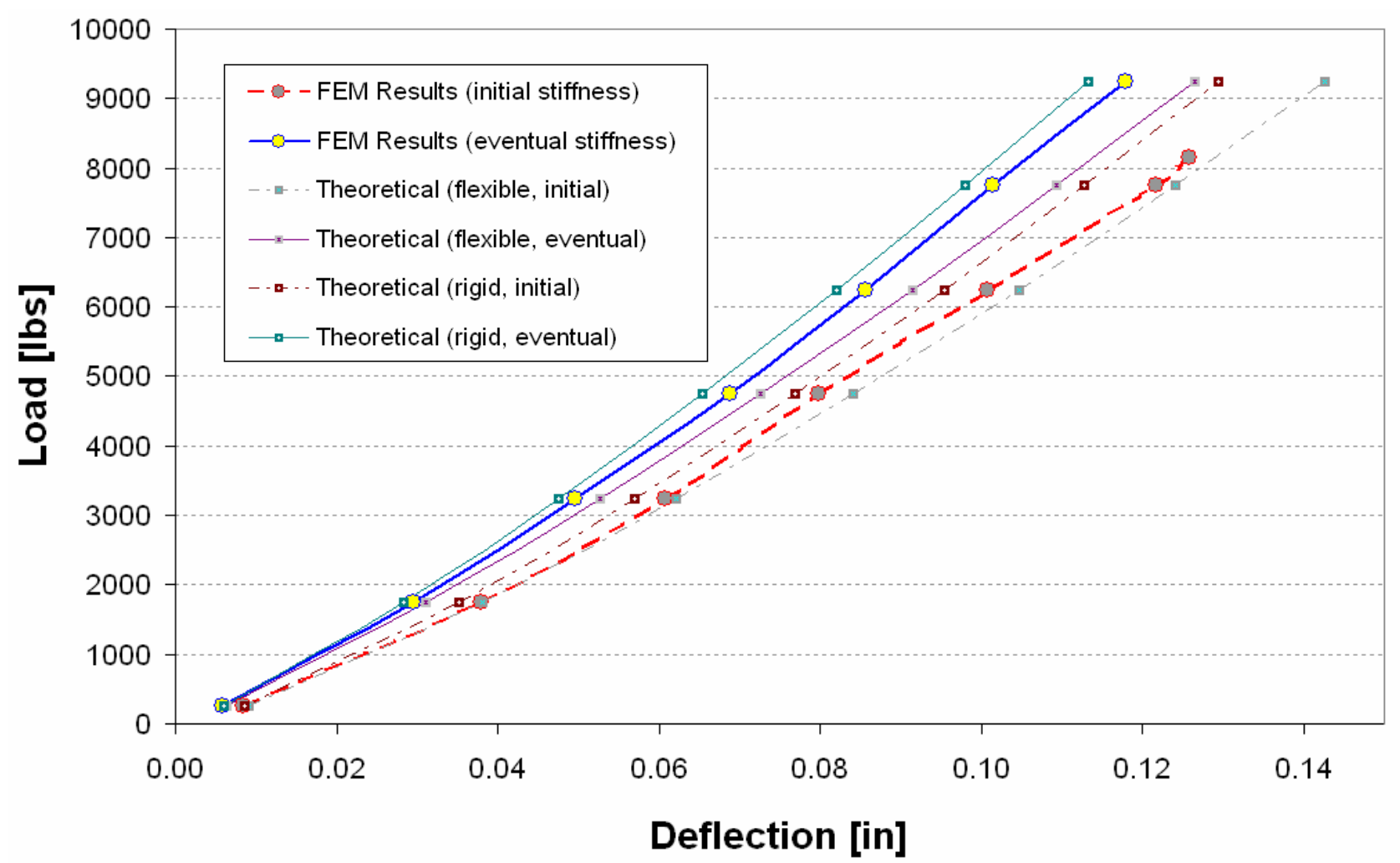

Figure 6.10: Comparisons of Theoretical Deflections with FEA Displacements at the Center of the Largest Loading Plate for Non-Repetitive Plate Bearing Tests of A-2-4 Subbases with Roadrain Using Two Different Roadrain Stiffnesses 


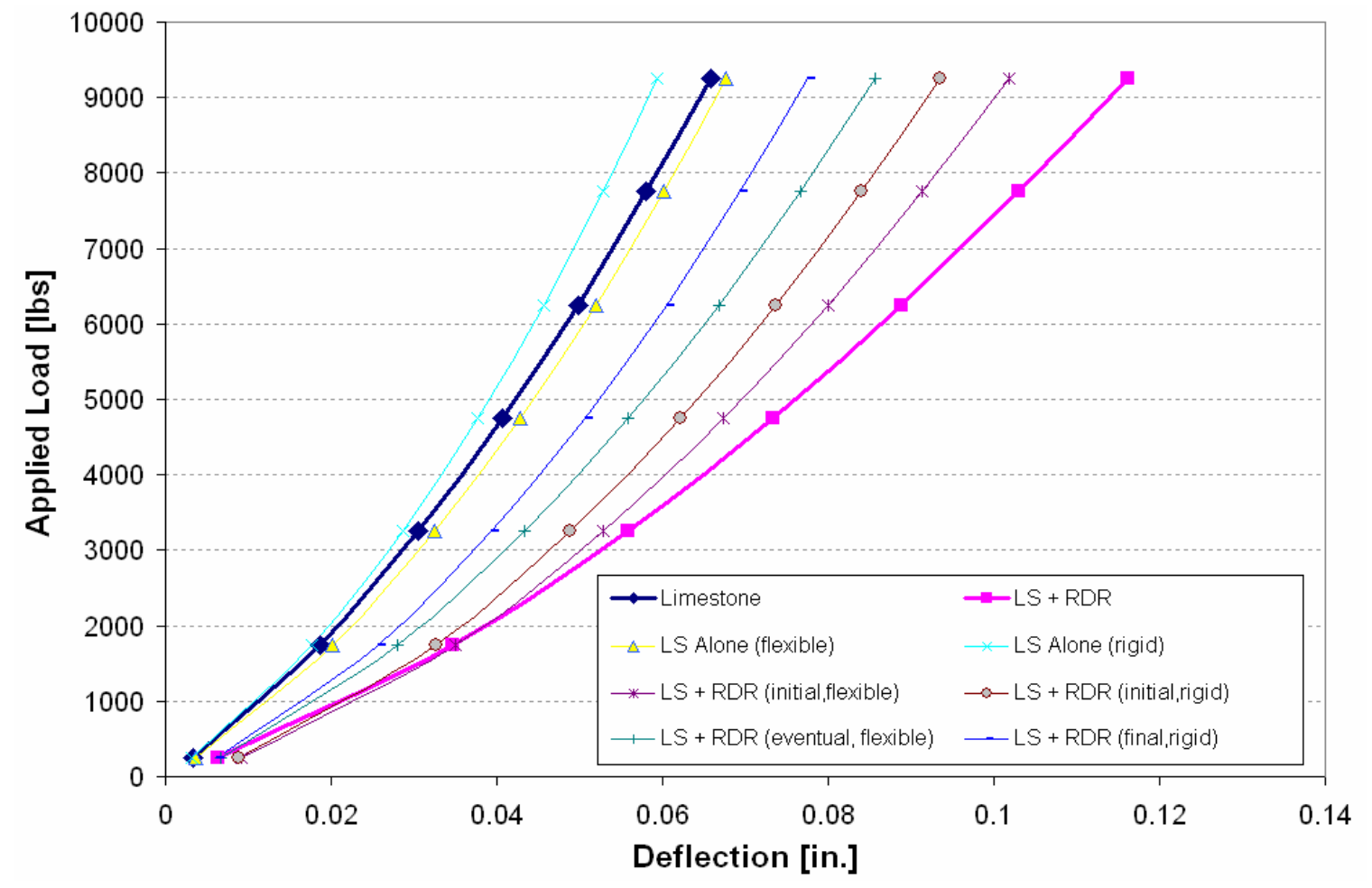

Figure 6.11: Comparisons of Theoretical Deflections with Experimental Displacements at the Center of the Largest Loading Plate for Non-Repetitive Plate Bearing Tests of \#57

Limestone Subbases with and without Roadrain

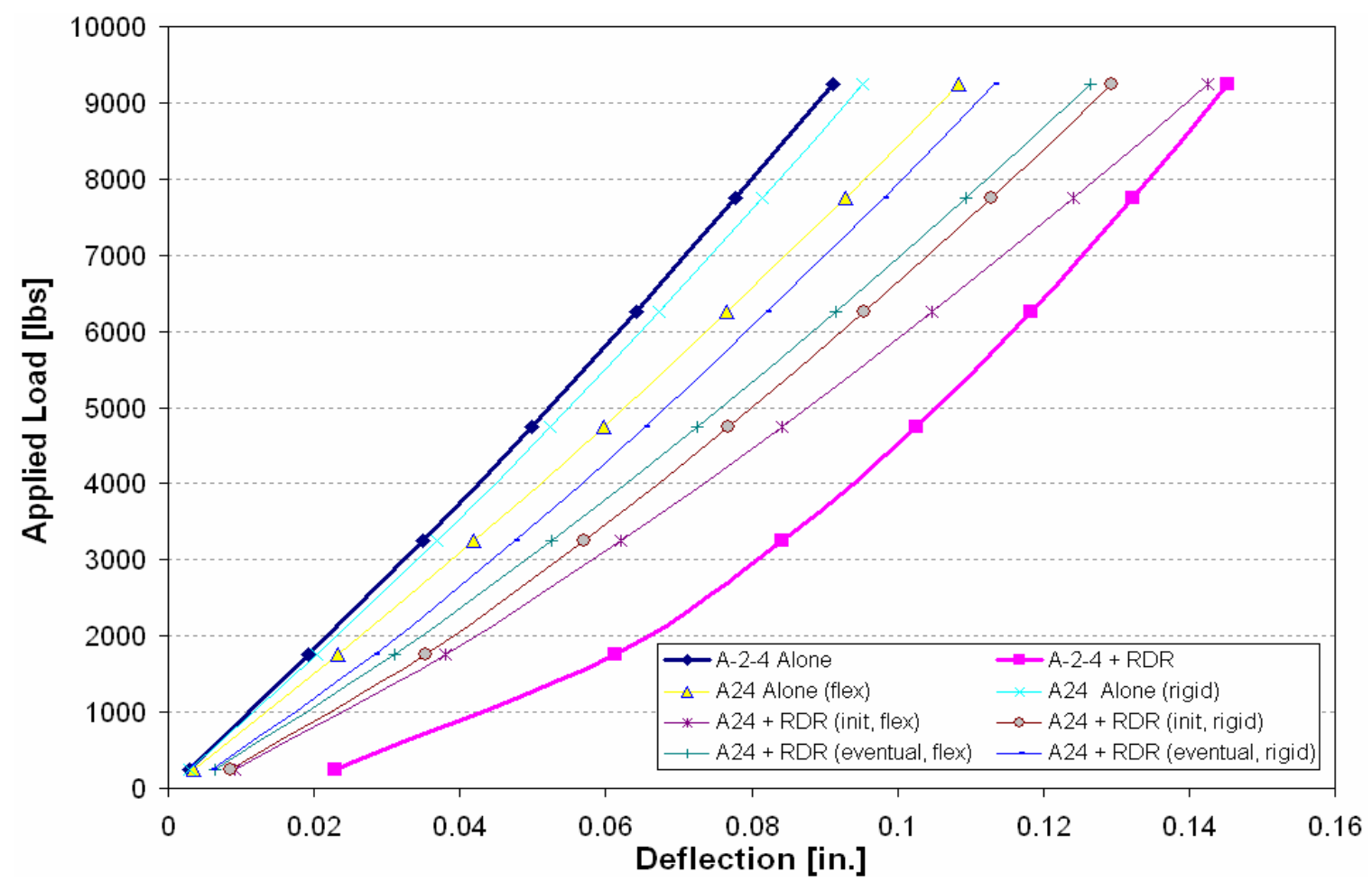

Figure 6.12: Comparisons of Theoretical Deflections with Experimental Displacements at the Center of the Largest Loading Plate for Non-Repetitive Plate Bearing Tests of A-2-4 Soil Subbases with and without Roadrain 


\section{CHAPTER 7 \\ CONCLUSIONS AND APPLICATIONS}

\subsection{Conclusions}

The results of this study would provide engineers with many of the theoretical tools necessary for implementing a drainage layer such as Roadrain into a PCC highway system. First of all, the establishment of various stress-strain relationships for the material when subjected to uniaxial compressive loading have been shown to be used both in theoretical and FE analyses. While the numerous unloading curves have not been discussed in great detail, these have the potential to be used in more complete analyses which incorporate the unloading behavior of this material. Also, the cyclic fatigue tests give some indication of how the material would behave throughout its lifetime in the field after numerous applications of traffic loading. Finally, the theoretical analysis technique used was shown through FE simulation to give reasonable results, so this simple procedure can be followed to estimate the results of the plate bearing tests in which Roadrain is placed directly on top of a soil support system. Conclusions drawn from these tests will be discussed in more detail throughout this section.

From the results of the vertical compression tests, much was learned about the vertical stiffness characteristics of the Roadrain geocomposite drainage layer when bonded to a concrete layer. These findings can be summarized as follows:

- As is the case with most soils, Roadrain undergoes an increase in stiffness with the re-application of a static load with a given magnitude. The rate of this increase is largest within the first few applications, after which the relative change in stiffness between load applications becomes small.

- The Roadrain deflection under an applied load is somewhat visco-elastic in that it has a time-dependent creep component. This contributes to the non-linearity of the stress-strain relationship of the Roadrain.

- Various stress-strain relationships for Roadrain were determined via uniaxial compression tests. Loading schemes were developed to account for the changes in these relationships due to the aforementioned effects from load repetition and duration. These were summarized in Table 3.6. 
- Overall, the Roadrain layer has a weak compressive stiffness in comparison with most other construction materials, especially at low stress levels, although it does increase in stiffness when subjected to higher stresses.

The other tests (cyclic fatigue and plate bearing) involved the use of Roadrain which was not bonded to a concrete layer. The findings of these are as follows:

- The distributions of the elastic and plastic strains during the cyclic fatigue tests varied depending upon the rate of application of loading. Generally, the elastic strain decreased with the rate of load application, and the plastic strain increased. Consequently, the resilient modulus increased with a loading rate increase, since it is inversely proportional to the elastic strain.

- Throughout the course of 1 million cycles of application of a 35 psi loading, Roadrain was seen to undergo a resilient modulus increase of more than $100 \%$. Also, the accumulated plastic deformation rose, on average, approximately $0.06 \mathrm{in}$. to levels approaching a maximum of almost 0.18 in.. The majority of these changes occurred within the first 500,000 cycles of load application, with less than a 7\% change in either characteristic occurring between 500,000 to $1,000,000$ cycles.

- The fact that the Roadrain was not bonded to concrete on one side is believed to have an effect on the stiffness characteristics. This is evident with the relatively lower initial stiffness from the cyclic fatigue test than what would be calculated from the vertical compression results. Also, from the plate bearing tests it was seen that the non-bonded Roadrain experienced higher deflections than what would be expected of bonded Roadrain at the same stress levels.

In addition to the vertical stiffness characterization, an analysis technique was proposed for predicting the results of the plate bearing tests, and thereby calculating the $k$-value for the soil. This technique was implemented using data from the non-repetitive plate bearing tests, as well as the Roadrain stiffnesses of Chapter 3. The results were then compared to the experimental data as well as Finite Element simulations of the tests scenarios. A good correlation was found between the FEA results and the theoretical 
equations, indicating that the theoretical calculations will give reasonable results provided that the material properties are properly defined. There were discrepancies between the theoretical and experimental results, however, when using the Roadrain stiffness equations from Chapter 3. The primary contributor to these discrepancies is believed to be the difference in stiffness between Roadrain layers which were not exposed to curing concrete and the Roadrain layers which were subjected to cement paste as concrete cured on the top layer of fabric. Also, the fact that the concrete would form to the texture of the Roadrain would provide more load distribution directly to the stronger ribs of the geonet core than the steel plates used for the Plate Bearing tests.

Other possible effects from the use of a Roadrain layer in PCC support systems would be:

- The increased drainage would increase the stiffness of the underlying soils in comparison to an undrained soil, as an increase in moisture content typically decreases the bearing capacity of a soil. This would most likely have the greatest effect during the spring thaw season when pavement support is usually significantly weaker due to the excessive water content.

- It was seen from the FE simulation of the plate bearing tests of an A-2-4 soil subbase plus a Roadrain layer that the differential settlement of the plate was reduced with the incorporation of the softer Roadrain layer as opposed to that when no Roadrain was present. This may indicate that the presence of this layer would in fact provide a cushioning effect, and therefore would help to provide a more even distribution of highway loads and may lead to a reduction of the slab stresses due to curling.

\subsection{Recommendations}

During all tests that were conducted, temperatures ranged from approximately $70^{\circ} \mathrm{F}$ to $80^{\circ} \mathrm{F}$. However, in the field Roadrain will be subjected to a much larger range of temperatures, depending on the geographical location; altering the temperature of the Roadrain would certainly change the stress-strain relationships developed in Chapter 3, and would most likely change the fatigue characteristics seen in Chapter 4. Particularly, at lower temperatures the material would most likely exhibit a higher overall stiffness, 
but at low temperatures the polymer may become brittle and more permanent damage may occur; at very high temperatures the material would probably have a slightly lower stiffness. The extent to which temperatures at the outer limits of what would be experienced in the field would affect the Roadrain's behavior is unknown, and would need to be investigated further. Also, in addition to temperature effects other environmental factors should be considered which may affect Roadrain's long-term behavior. For example, some chemicals or deicing salts may cause the eventual oxidation and/or structural breakdown of the plastic Geonet core. Also, the susceptibility of the Roadrain to alkaline attack and changes in properties due to freeze-thaw cycles should be considered.

There were some assumptions made which simplified the theoretical analysis that should be investigated more thoroughly. Specifically, it was assumed that (1) shear transfer in the Roadrain layer is negligible, so its presence would not change the area of loading at the subbase interface, and (2) any friction between the bottom of the Roadrain layer and the subbase would not significantly alter the stresses at the top of the subbase. While these effects are not believed to be significant when dealing with the case of a plate bearing test conducted using a Roadrain layer of Finite length, these constraints may play a larger role when dealing with the more continuous system of a PCC highway pavement system. It is believed that accounting for these effects would cause an overall reduction in the deflection of the support system, though, so using the current technique would give a conservative estimate of the overall stiffness.

Another consideration for using a Roadrain layer would be the resulting change in shear transfer capabilities of the pavement at the locations of joints. A relatively small initial load transfer capability is expected at the joints due to the low initial stiffness of the Roadrain; however, a long-term effect of decreased erosion from pumping, if present, may compensate for this effect by increasing the sustainability of the initial level of load transfer. Also, the environmental factors mentioned above which may eventually change the Roadrain's properties would also affect the load transfer efficiency of the pavement structure. The load transfer efficiency changes are believed to have more potential for being evident in the cases of JPCPs than in CRCPs, since JPCPs generally have larger average crack widths. These effects should be thoroughly investigated. 


\section{REFERENCES}

AASHTO Guide for Design of Pavement Structures, 1993. American Association of State Highway and Transportation Officials, Washington, DC.

ABAQUS, 2004. Users Manual, Version 6.4.3.

ACPA, 1984. Construction and Rehabilitation of Concrete Pavement, Manual and Training Course, Contract No. DTFH-61-81-C-00051.

ACPA, 1995. Subgrades \& Subbases for Concrete Pavements; Publication TB011.02P.

Algor, 1990. ViziCad Plus, Modeling for Finite Element Analysis; Algor Interactive Systems, Inc, Pittsburgh, PA.

ASCE, 1994. Settlement Analysis, Technical Engineering and Design Guides as Adapted from the US Army Corps of Engineers, No. 9; ASCE Press, New York, NY.

Asphalt Institute, 1981. Thickness Design, Asphalt Pavements for Highways \& Streets; Asphalt Institute Manual Series No. 1 (MS-1).

ASTM C469, Standard Test Method for Static Modulus of Elasticity and Poisson's Ratio of Concrete in Compression, American Society for Testing and Materials.

ASTM D1195, Standard Method for Repetitive Static Plate Load Tests of Soils and Flexible Pavement Components, for Use in Evaluation and Design of Airport and Highway Pavements, American Society for Testing and Materials.

ASTM D1196, Standard Method for Nonrepetitive Static Plate Load Tests of Soils and Flexible Pavement Components, for Use in Evaluation and Design of Airport and Highway Pavements, American Society for Testing and Materials.

ASTM D3080, Standard Test Method for Direct Shear Test of Soils Under Consolidated Drained Conditions, American Society for Testing and Materials.

ASTM D4318, Standard Test Method for Liquid Limit, Plastic Limit, and Plasticity Index of Soils, American Society for Testing and Materials.

ASTM D6913, Standard Test Methods for Particle-Size Distribution (Gradation) of Soils Using Sieve Analysis, American Society for Testing and Materials.

Bowles, J. E., 1974. Analytical and Computer Methods in Foundation Engineering; McGraw-Hill, New York, NY. 
Chen, H. L., T. H. Schell, and J. G. Sweet, 2002. "Field Study of the Early-Age Behavior of Jointed Plain Concrete Pavements," in Concrete: Material Science to Application, A Tribute to Surendra P. Shah, ACI Special Publication SP206-15.

Childs, L. D. and P. J. Nussbaum, 1962. "Pressures at Foundation Soil Interfaces Under Loaded Concrete and Soil-Cement Highway Slabs," Proceedings of the American Society for Testing and Materials, Vol. 63, pp 1243-1263.

Consedine, R. L. (editor), "Concrete Roads Claim Advantages," Aggregates and Roadbuilding Magazine; http://www.rocktoroad.com/concreteroads.html, Westmount, Quebec.

Cook, J. B., 2004. Frictional Resistance of Concrete on a Geocomposite Material, Thesis, West Virginia University.

Das, B. M., 1998. Principles of Geotechnical Engineering; $4^{\text {th }}$ edition, PWS Publishing, Boston, MA.

Das, B. M., 1999. Shallow Foundations, Bearing Capacity and Settlement; CRC Press, Boca Raton, FL.

ELE International, 1999. ELE Construction Materials Testing Equipment Catalog, $5^{\text {th }}$ edition; ELE International, Lake Bluff.

Farny, J. A., 2001. Concrete Floors on Ground, EB075, Portland Cement Association.

Federal Highway Administration (FHWA). 1992. Report on the 1992 U.S. Tour of European Concrete Highways. FHWA-SA-93-012. Federal Highway Administration, Washington, D.C.

FM 5-527. Florida Method of Test for Nonrepetitive Static Plate Load Test of Soils and Flexible Pavement Components.

Gharaibeh, N. G., M. I. Darter, and L. B. Heckel. 1999. Field Performance of CRCP in Illinois. Preprint No. 990731. Seventy-Eighth Annual Meeting of the Transportation Research Board, Washington, DC.

Grater, S. F. and B. F. McCullough, 1994. Analysis of Jointed Concrete Pavement; Report No. FHWA/TX-95+1244-10.

Huang, Y. H., 2004. Pavement Analysis and Design; Pearson Prentice Hall, Upper Saddle River, NJ.

Masada, T., and S. M. Sargand, 2001. Laboratory Characterization of Materials \& Data Management for Ohio-SHRP Projects (US 23); Report No FHWA/OH-2001/07. 
Mindess, S., and J. F. Young, 1981. Concrete; Prentice-Hall, Englewood Cliffs, NJ.

PCA, 1984. Thickness Design for Concrete Highway and Street Pavements; Portland Cement Association.

PCA, 1992. PCA Soil Primer; Portland Cement Association.

Ping, W.V. and L. Ge. 1997. "Field Verification of Laboratory Resilient Modulus Measurements on Subgrade Soils," in Transportation Research Record 1577, TRB, National Research Council, Washington, D.C., pp. 53-61.

Westergaard, H. M., April 1926. "Stresses in Concrete Pavements Computed by Theoretical Analysis," Public Roads, Vol. 7, No. 2, pages 25 to 35.

Yang, N. C., 1972. Design of Functional Pavements; McGraw-Hill, New York. 


\section{APPENDIX A}

Raw Data Collected from the Repetitive Load-Release Vertical Compression Tests 




Figure A.1: Raw Data from Repetitive Load-Release Vertical Compression Test of Cylinder C2WC04 \#1

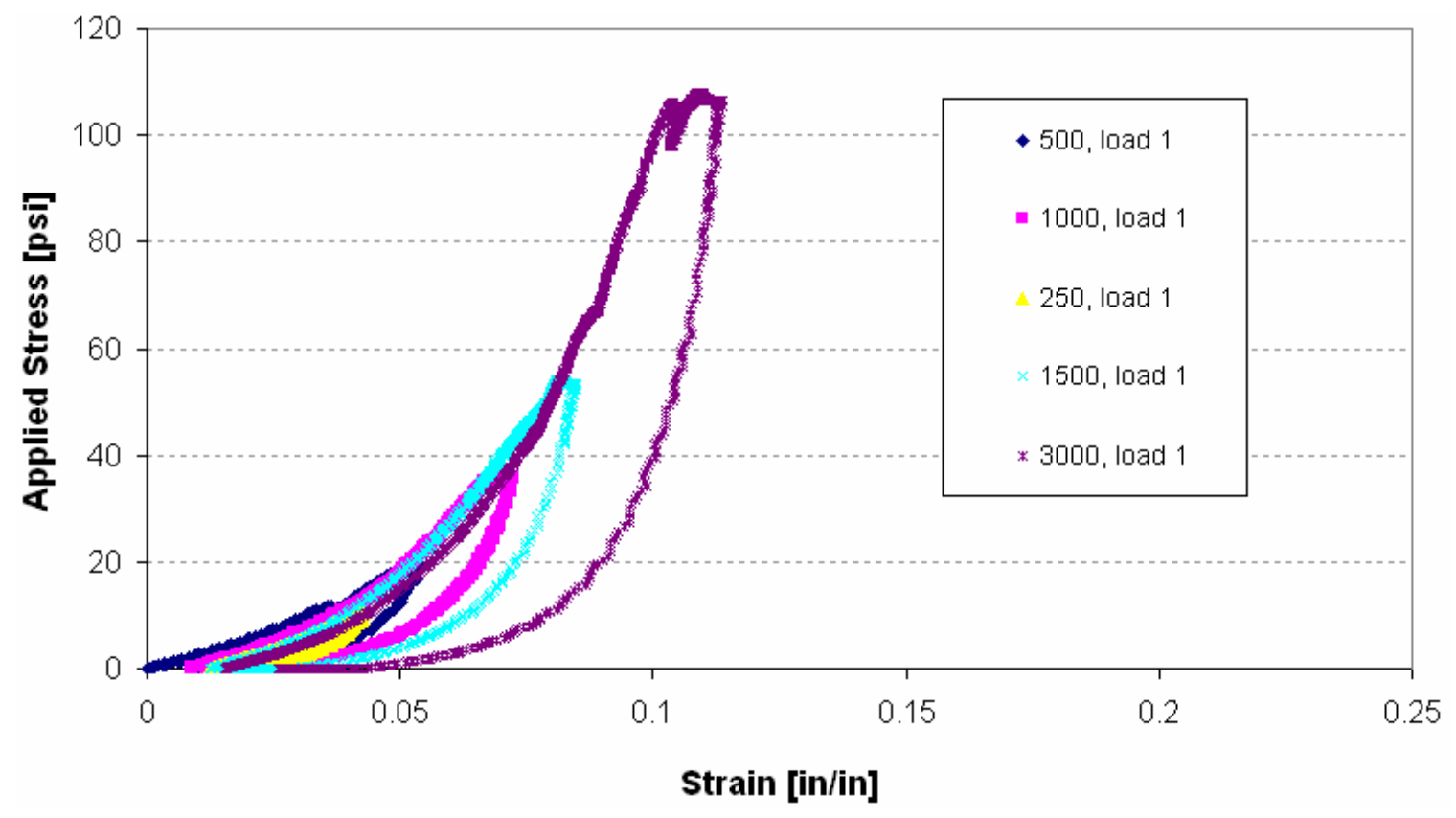

Figure A.2: Raw Data from Repetitive Load-Release Vertical Compression Test of Cylinder C2WC04 \#1b 


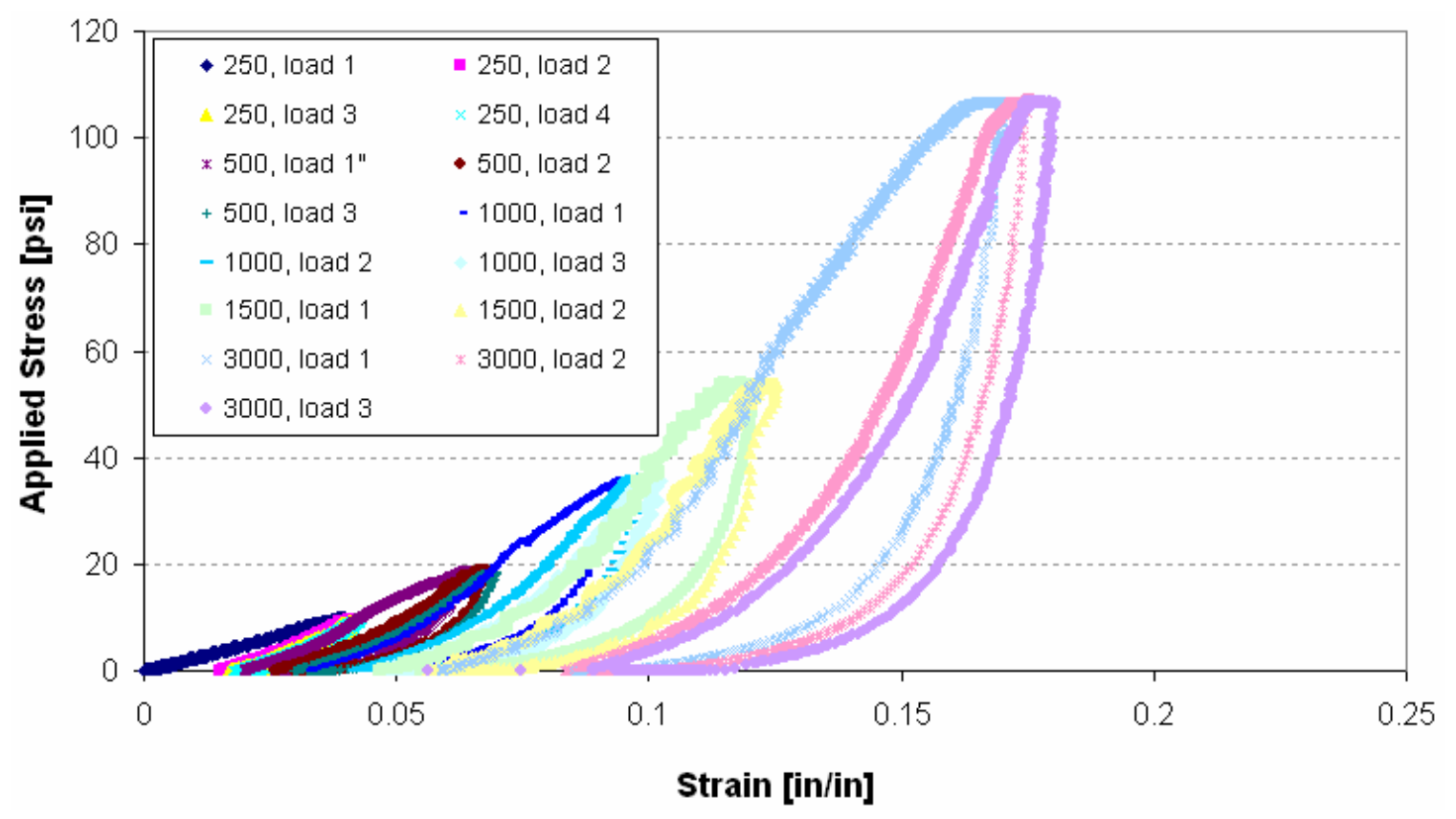

Figure A.3: Raw Data from Repetitive Load-Release Vertical Compression Test of Cylinder C2WC04 \#3

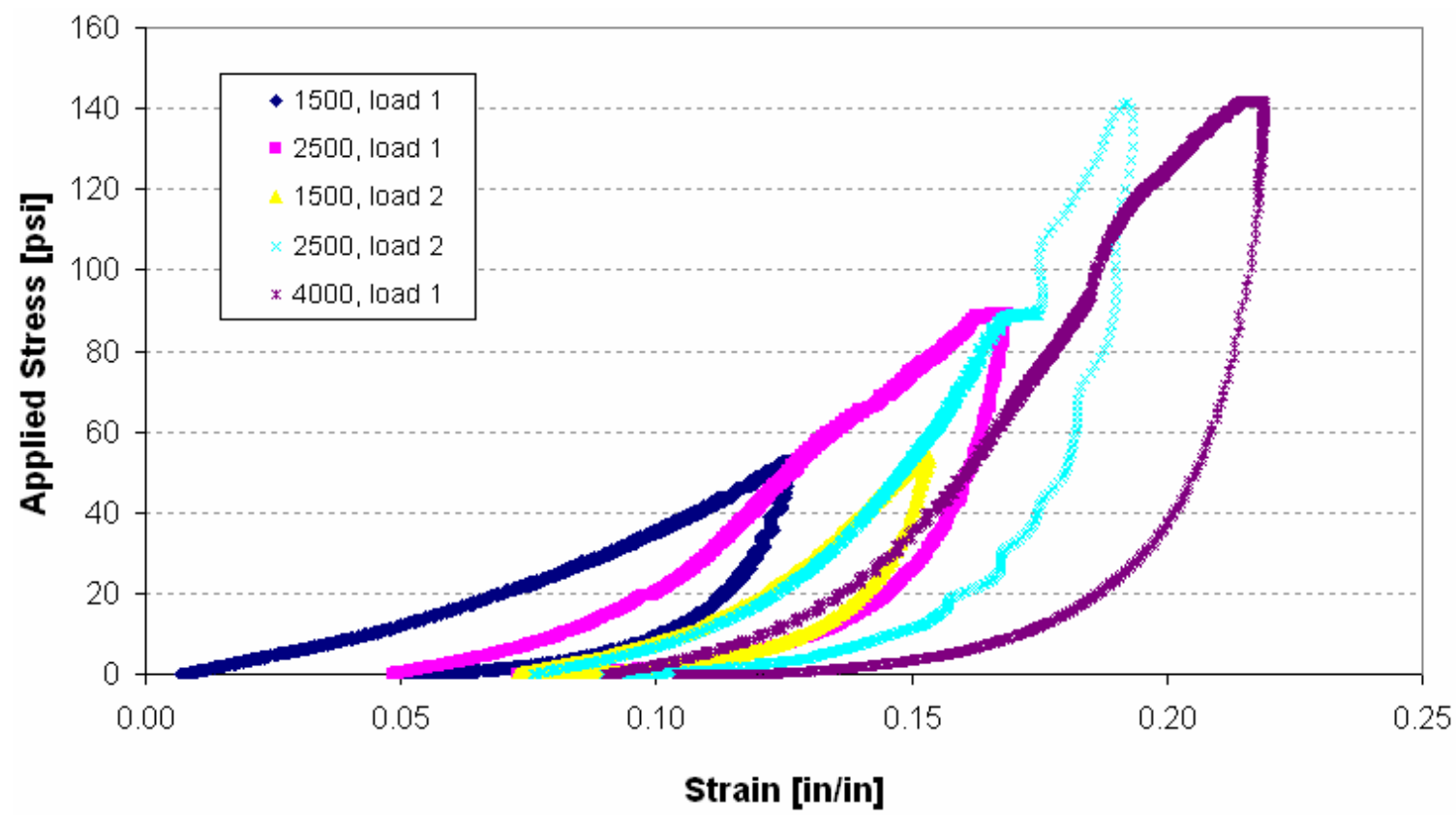

Figure A.4: Raw Data from Repetitive Load-Release Vertical Compression Test of Cylinder C2WC057 \#2 


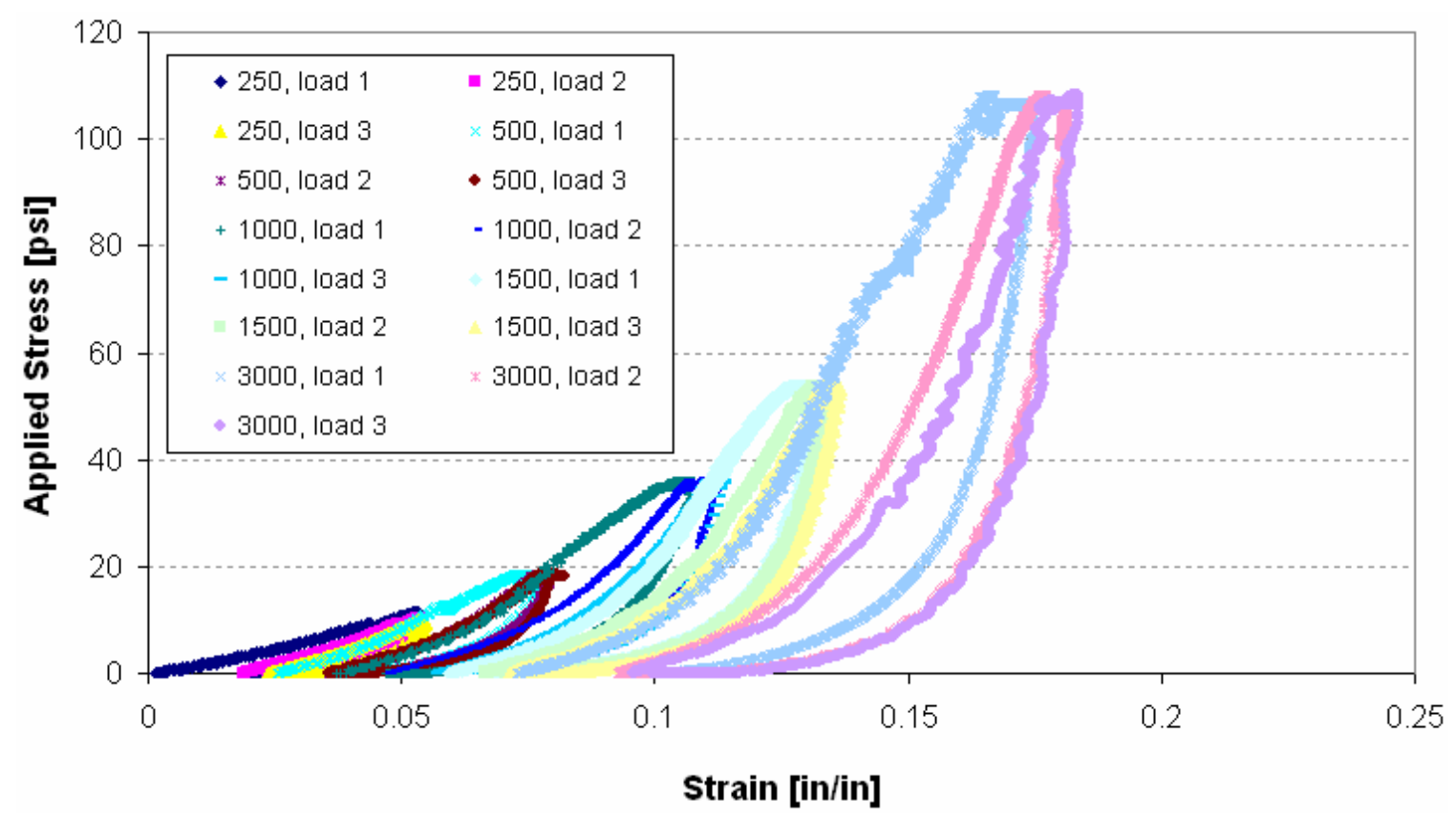

Figure A.4: Raw Data from Repetitive Load-Release Vertical Compression Test of Cylinder C2WC057 \#3 


\section{APPENDIX B}

Raw Data from Repetitive Plate Bearing Tests and Release Curves from All Plate Bearing Tests 


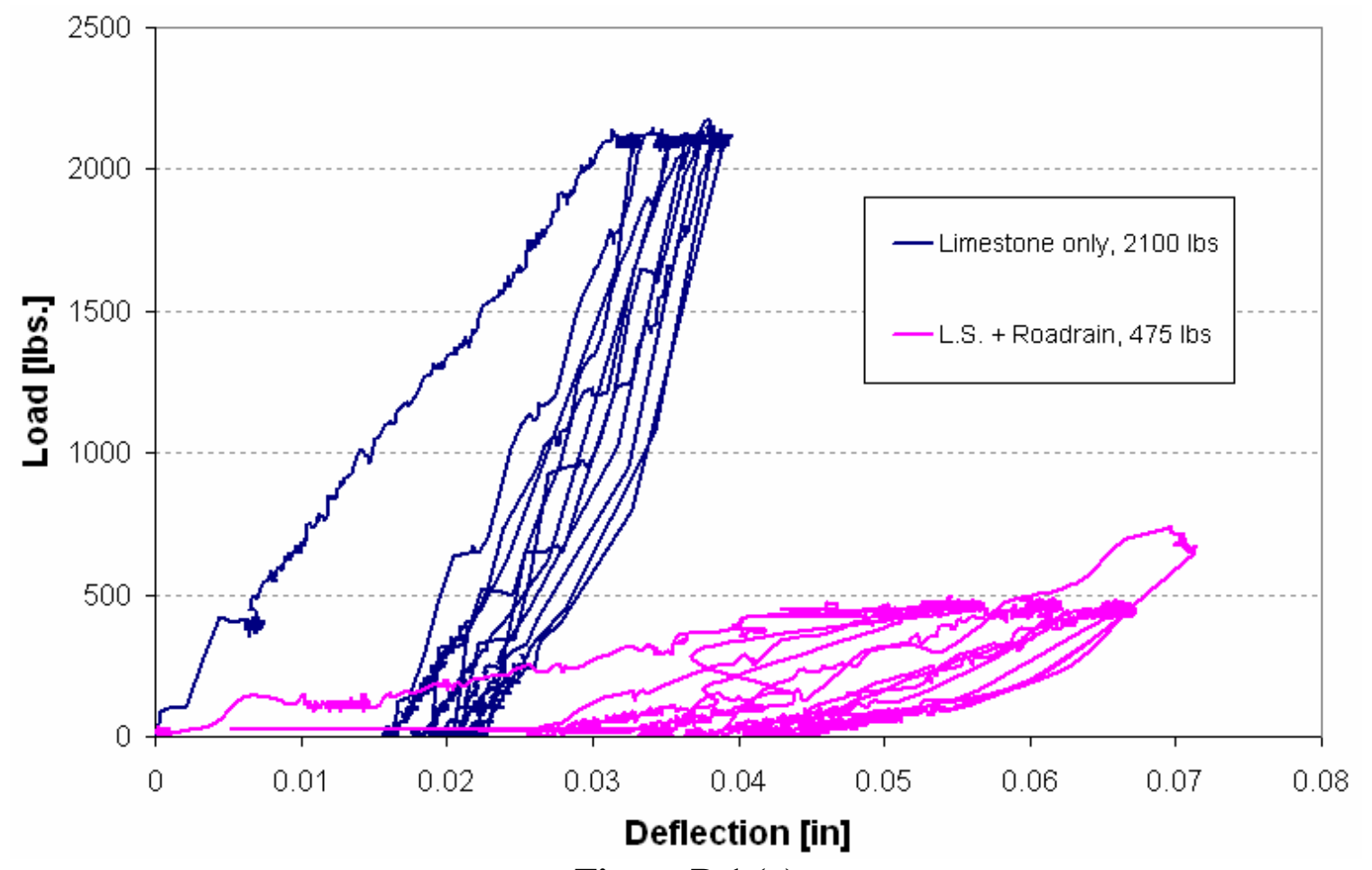

Figure B.1 (a)

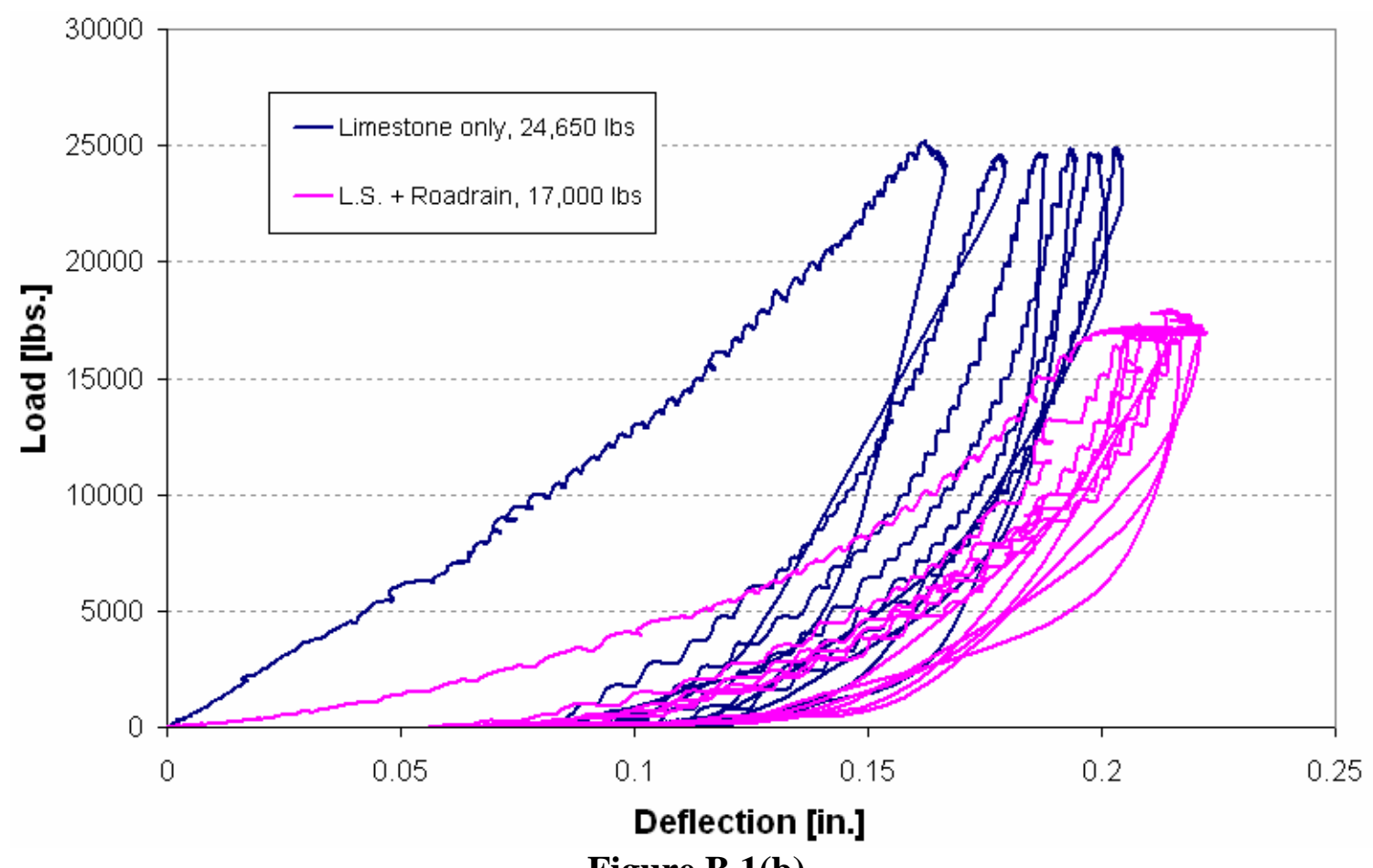

Figure B.1(b) 




(c)

Figure B.1: Raw Data Collected for the Repetitive Static Plate Bearing Tests of \#57 Subbases Both with and without a Roadrain Layer. Tested at (a) Small, (b) Medium, and (c) Large Load Magnitudes 


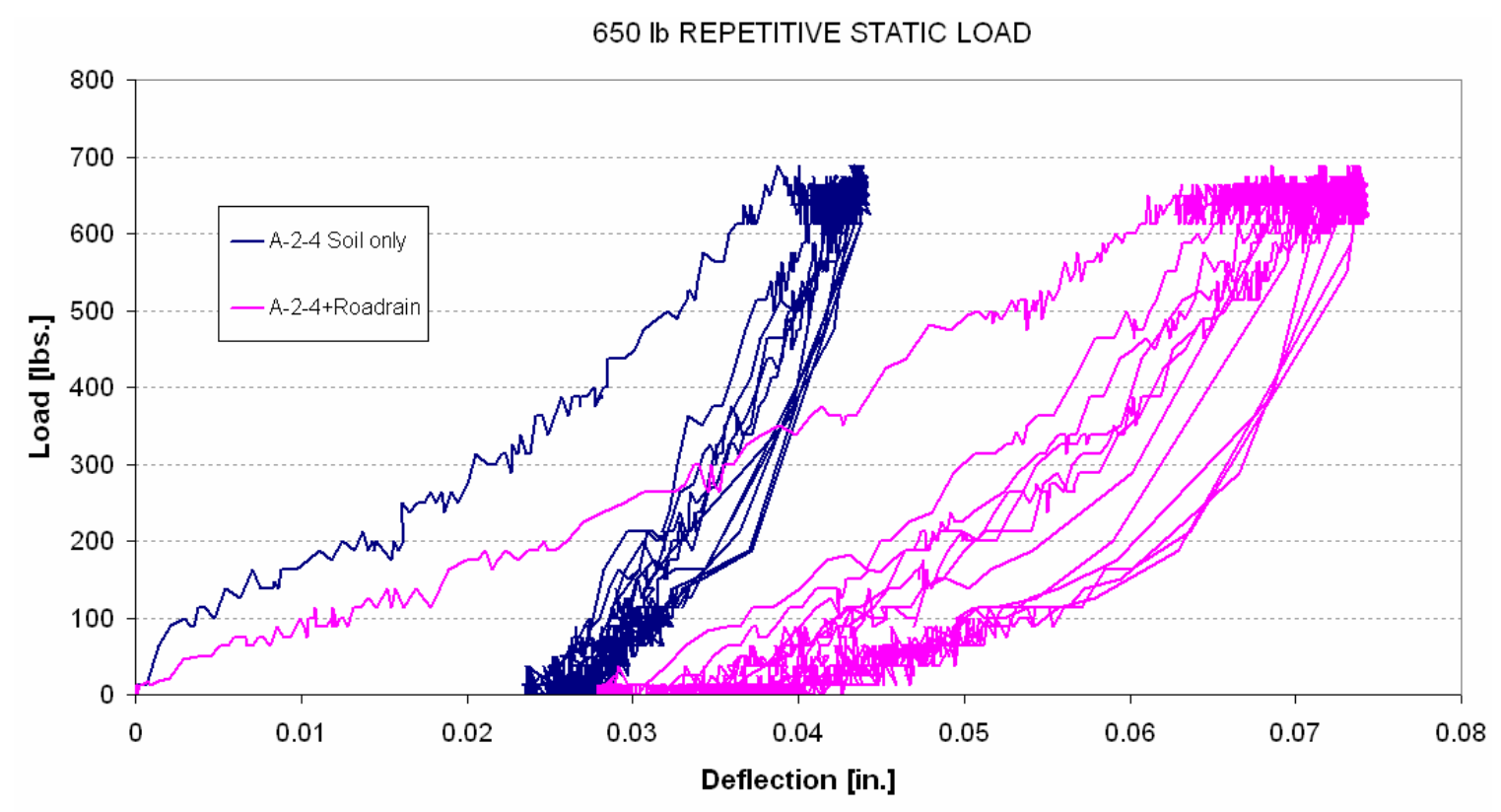

Figure B.2(a)

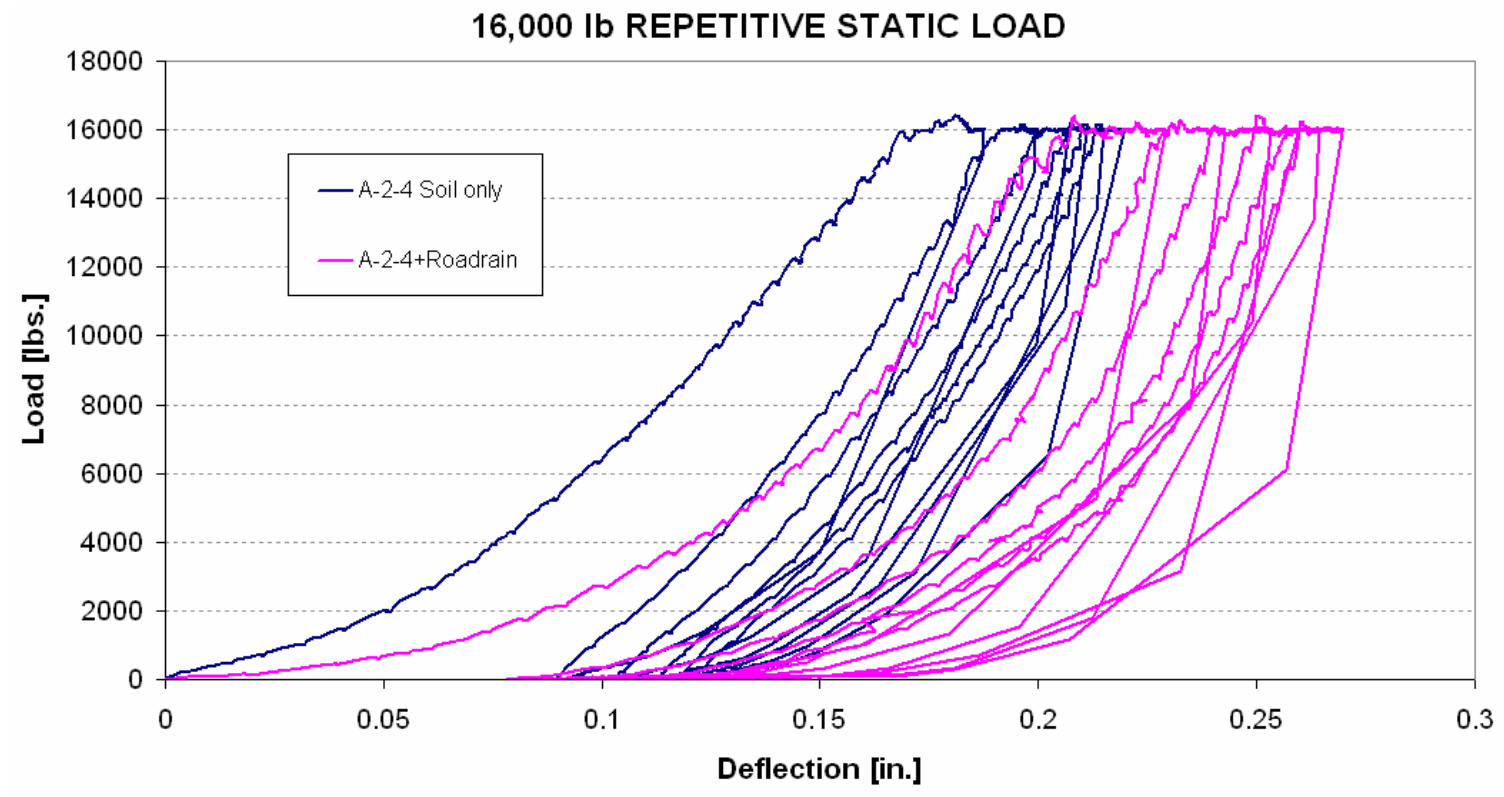

Figure B.2(b) 
35,000 lb REPETITIVE STATIC LOAD

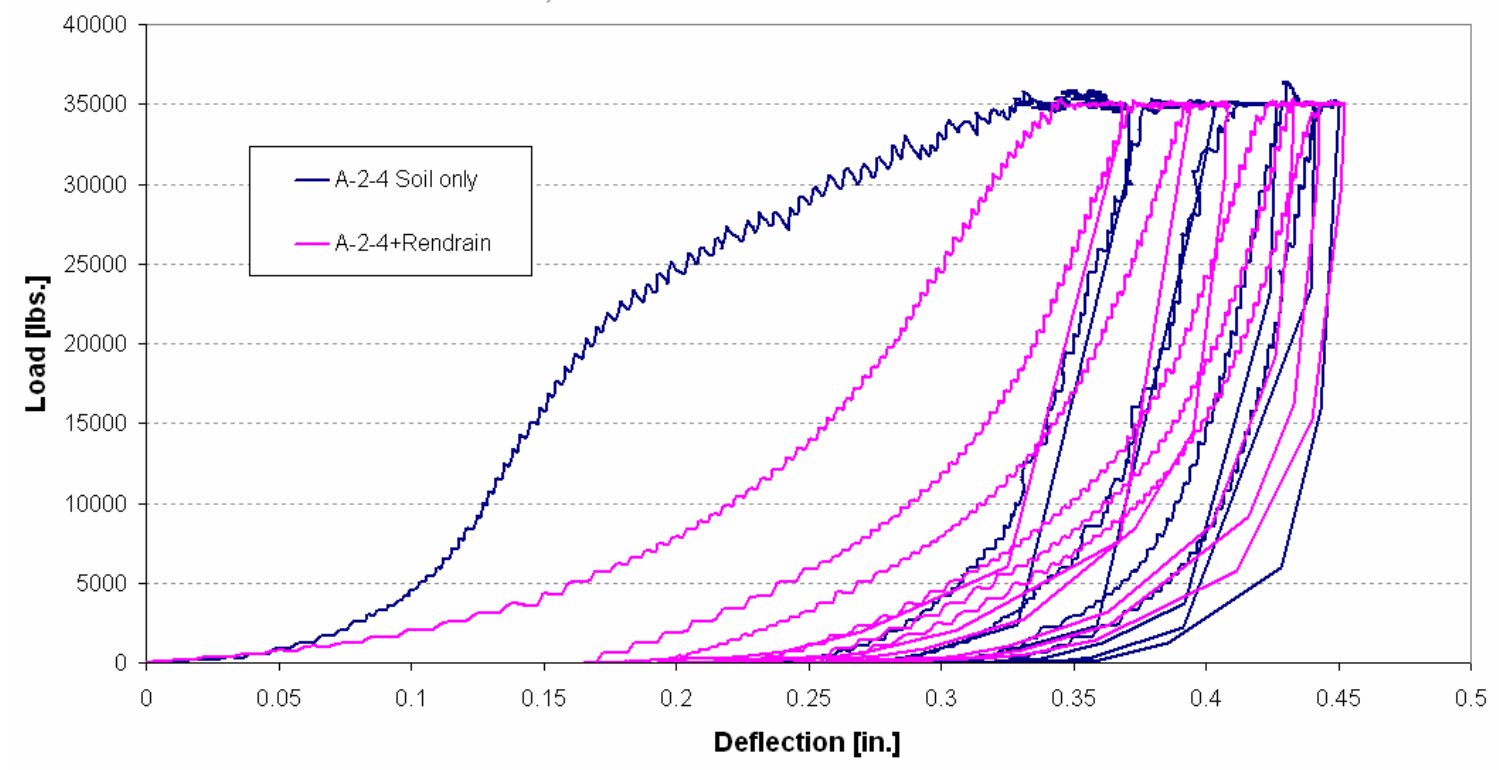

(c)

Figure B.2: Raw Data Collected for the Repetitive Static Plate Bearing Tests of A-2-4 Soil Subbases Both with and without a Roadrain Layer. Tested at (a) Small, (b) Medium, and (c) Large Load Magnitudes 


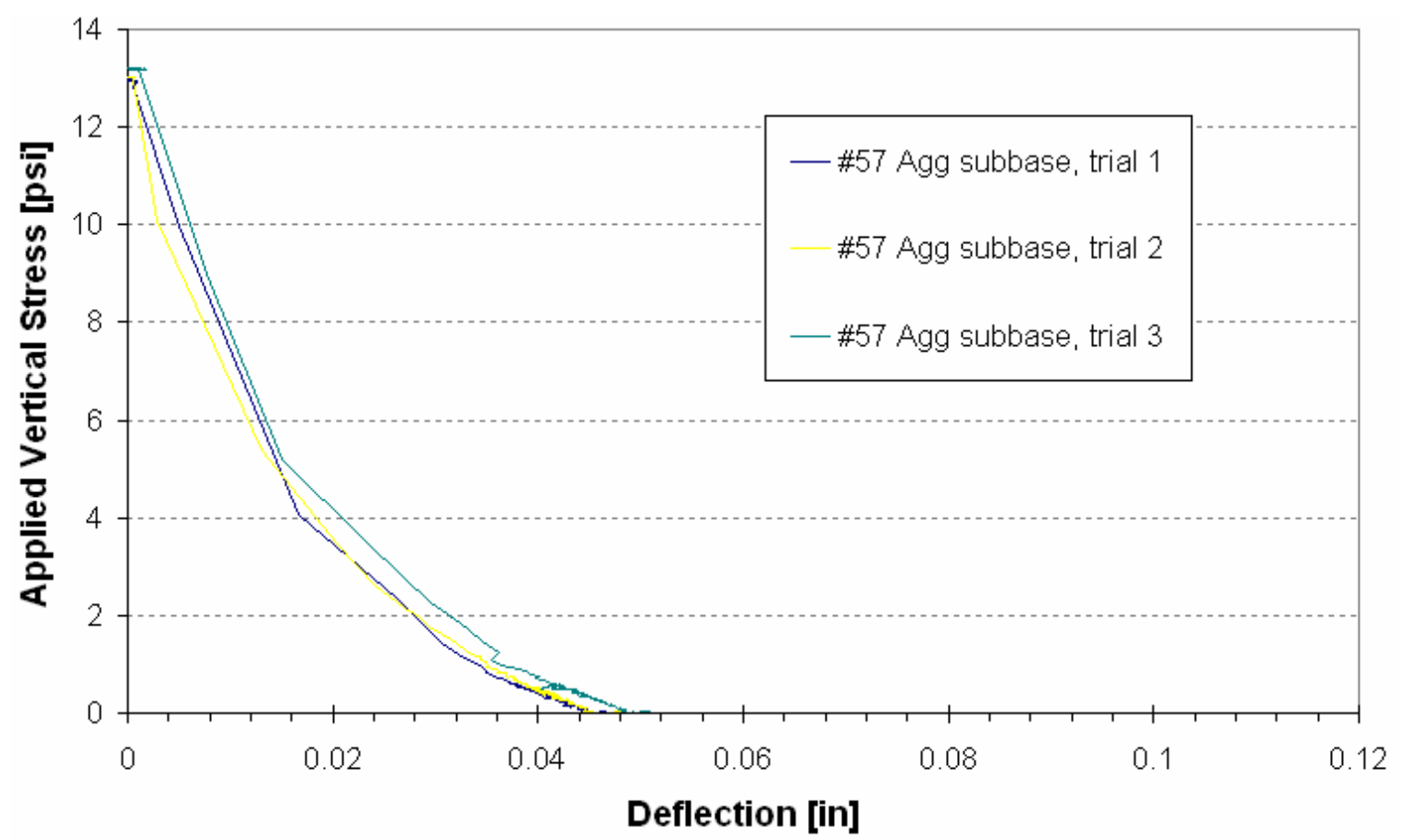

Figure B.3(a)

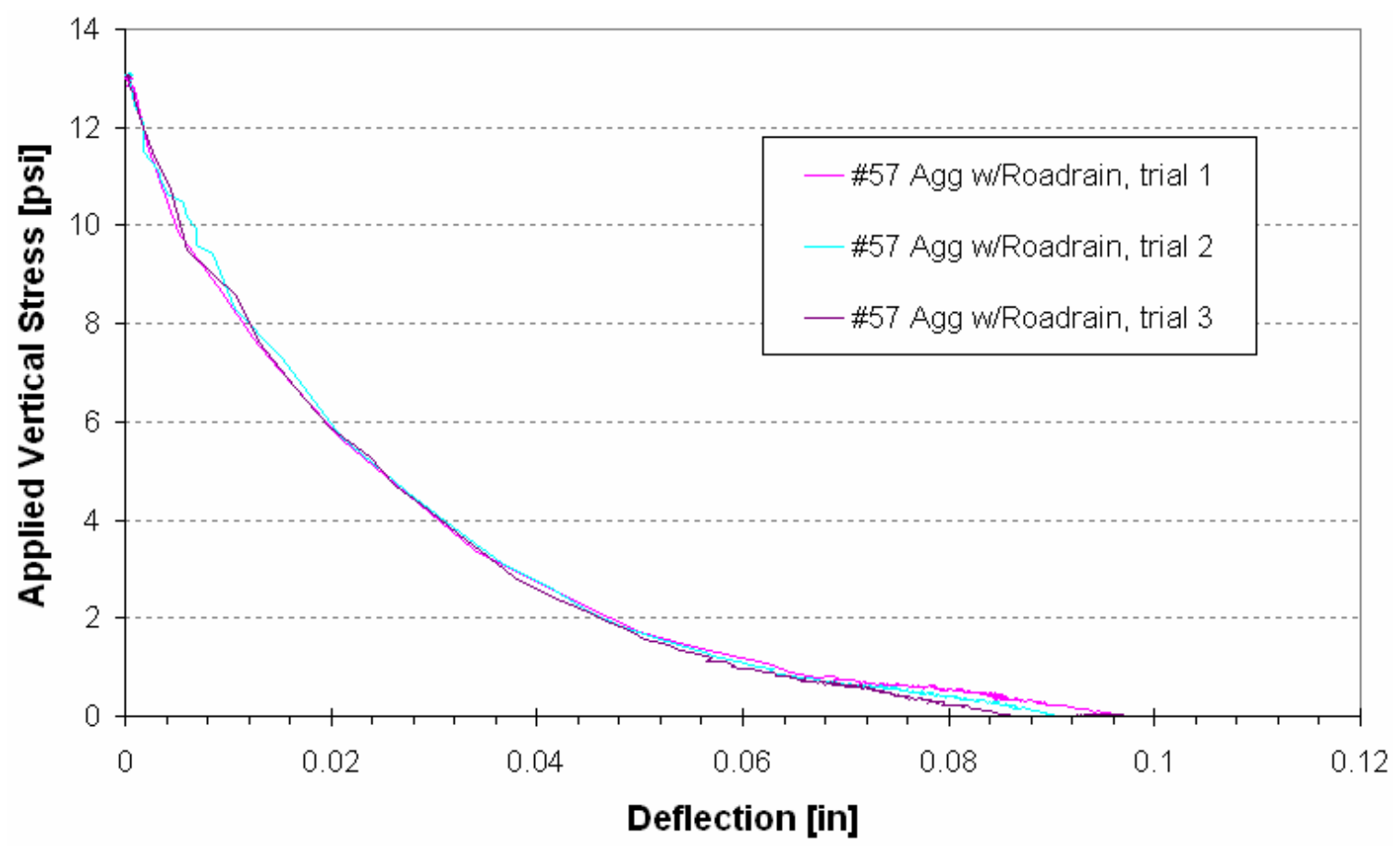

Figure B.3(b) 


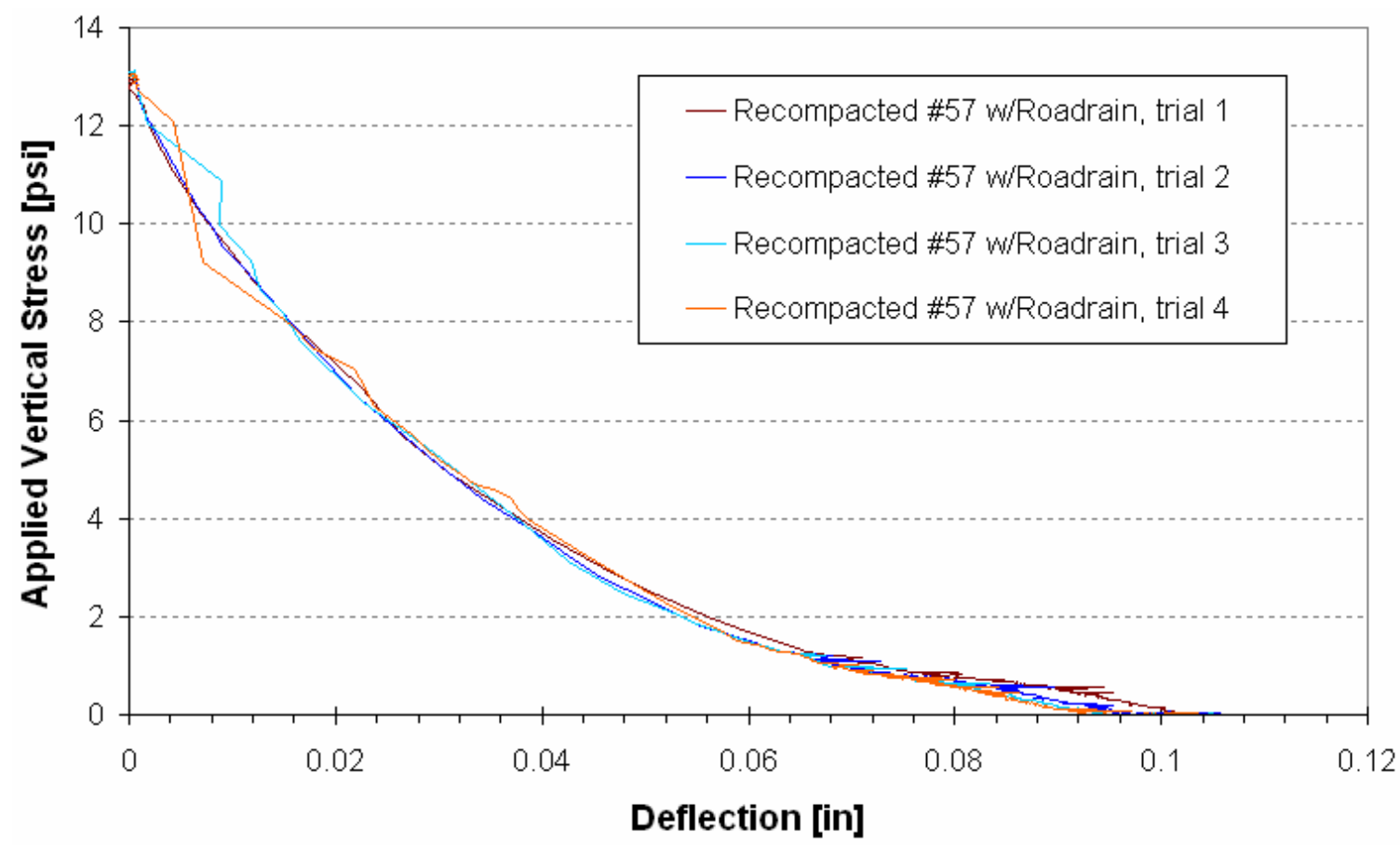

(c)

Figure B.3: Rebound Curves for all Non-Repetitive Static Plate Bearing Tests Using a \#57 Limestone Aggregate Subbase: (a) Subbase Alone, (b) Subbase Plus Roadrain, and (c) Recompacted Subbase Plus Roadrain. 


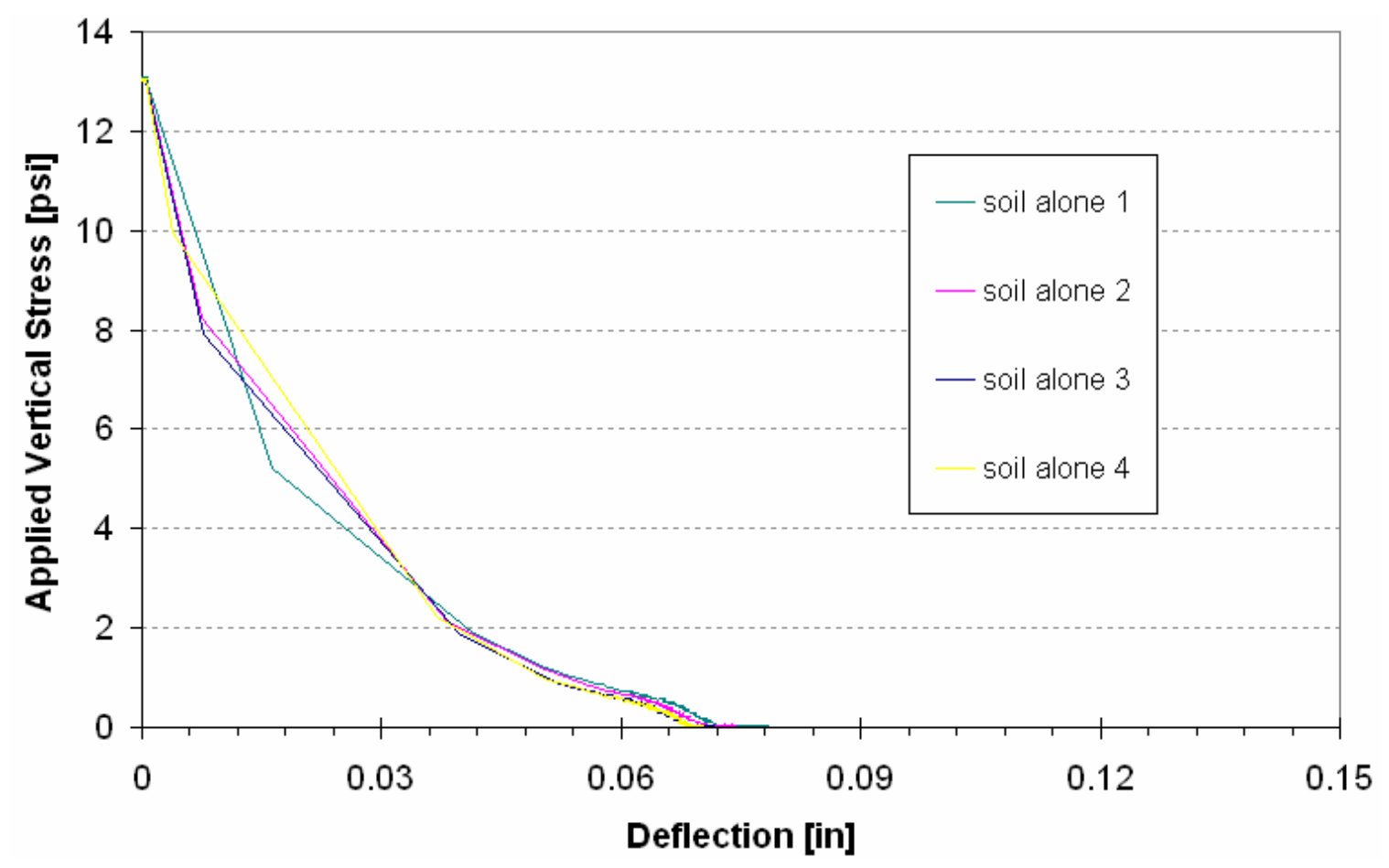

Figure B.4(a)

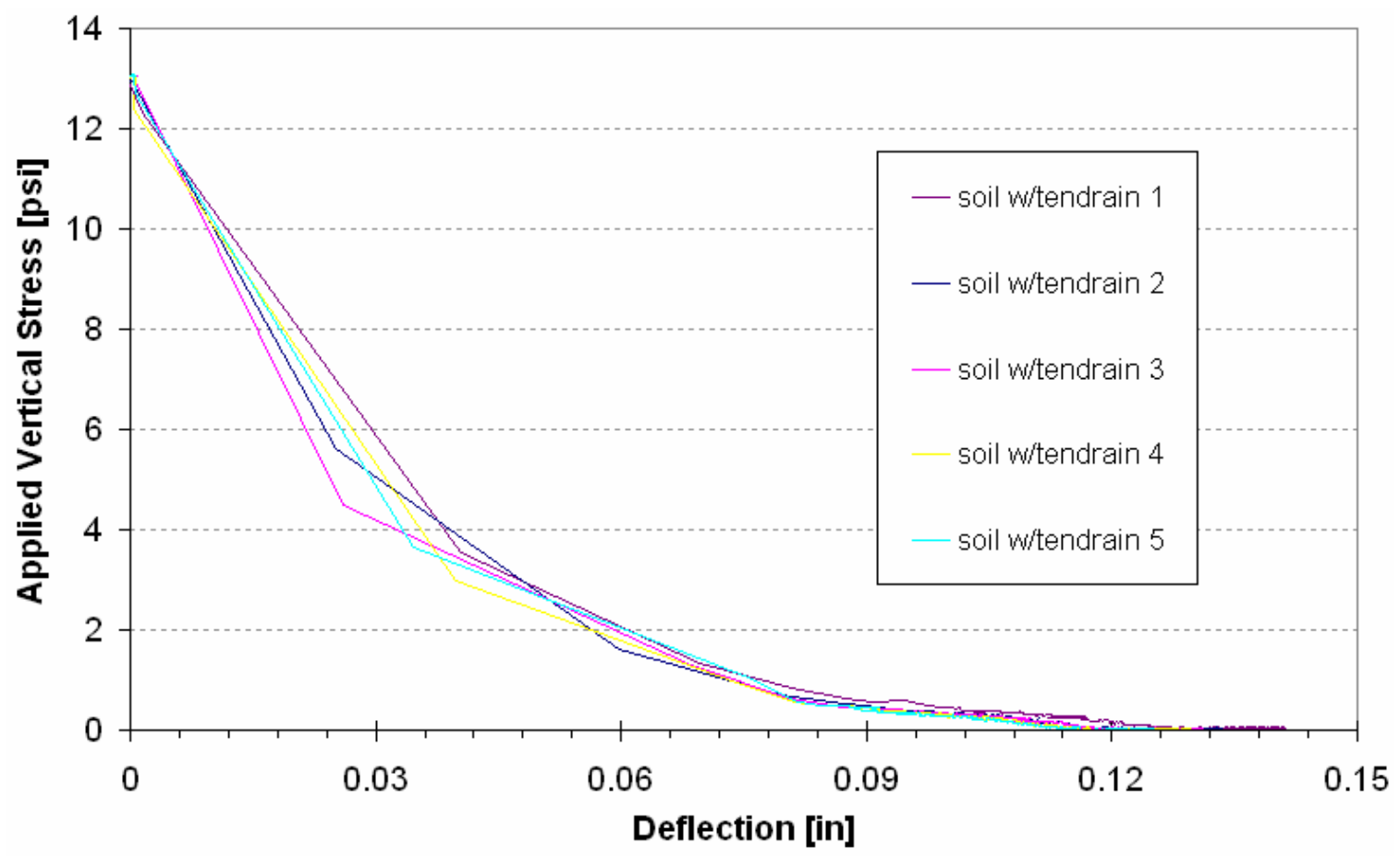

Figure B.4(b) 


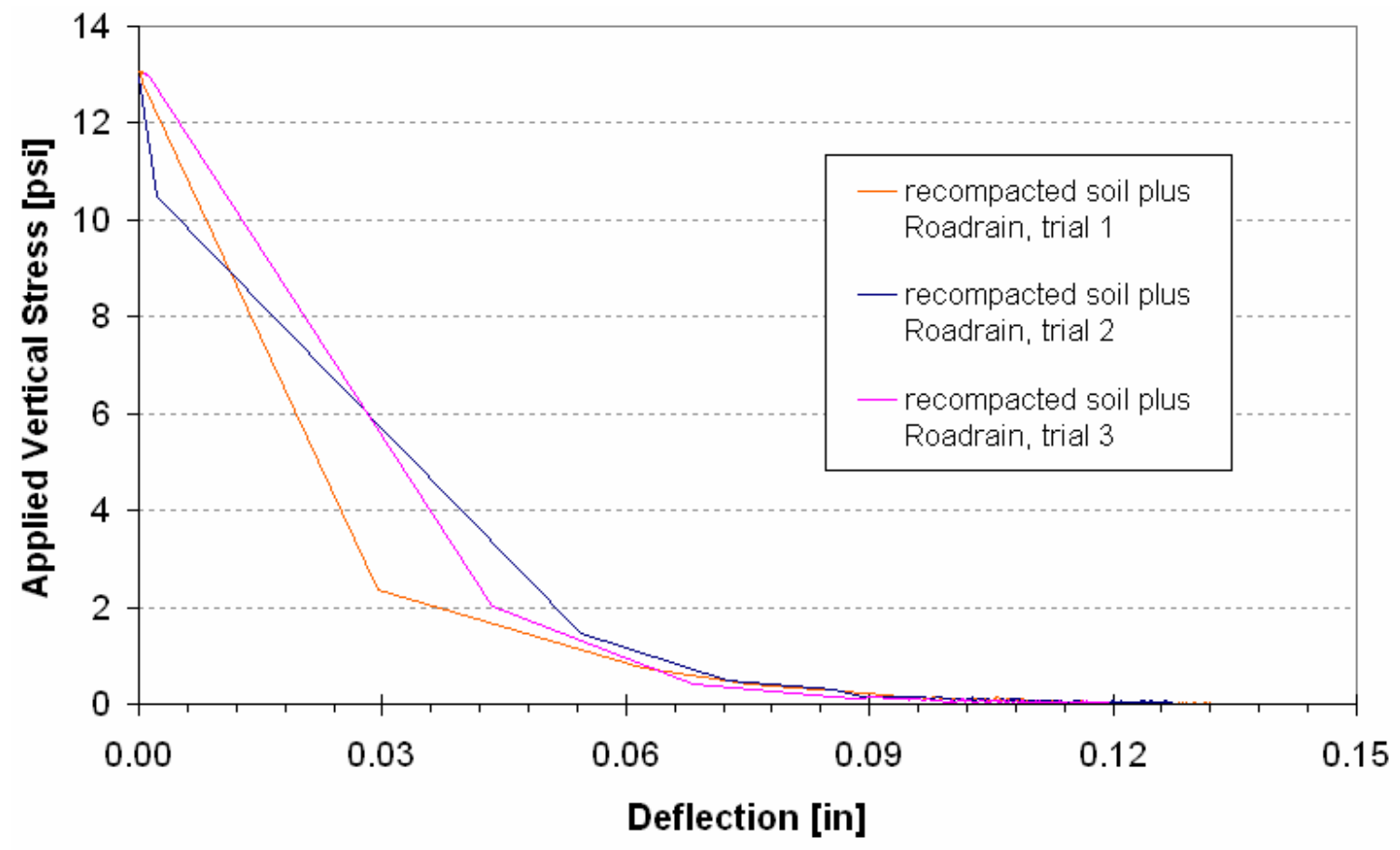

(c)

Figure B.4: Rebound Curves for all Non-Repetitive Static Plate Bearing Tests Using an A-2-4 Soil Subbase: (a) Subbase Alone, (b) Subbase Plus Roadrain, and (c) Recompacted Subbase Plus Roadrain. 
LIMESTONE SUBBASE, $24,650 \mathrm{lb}$. MAX APPLIED LOAD

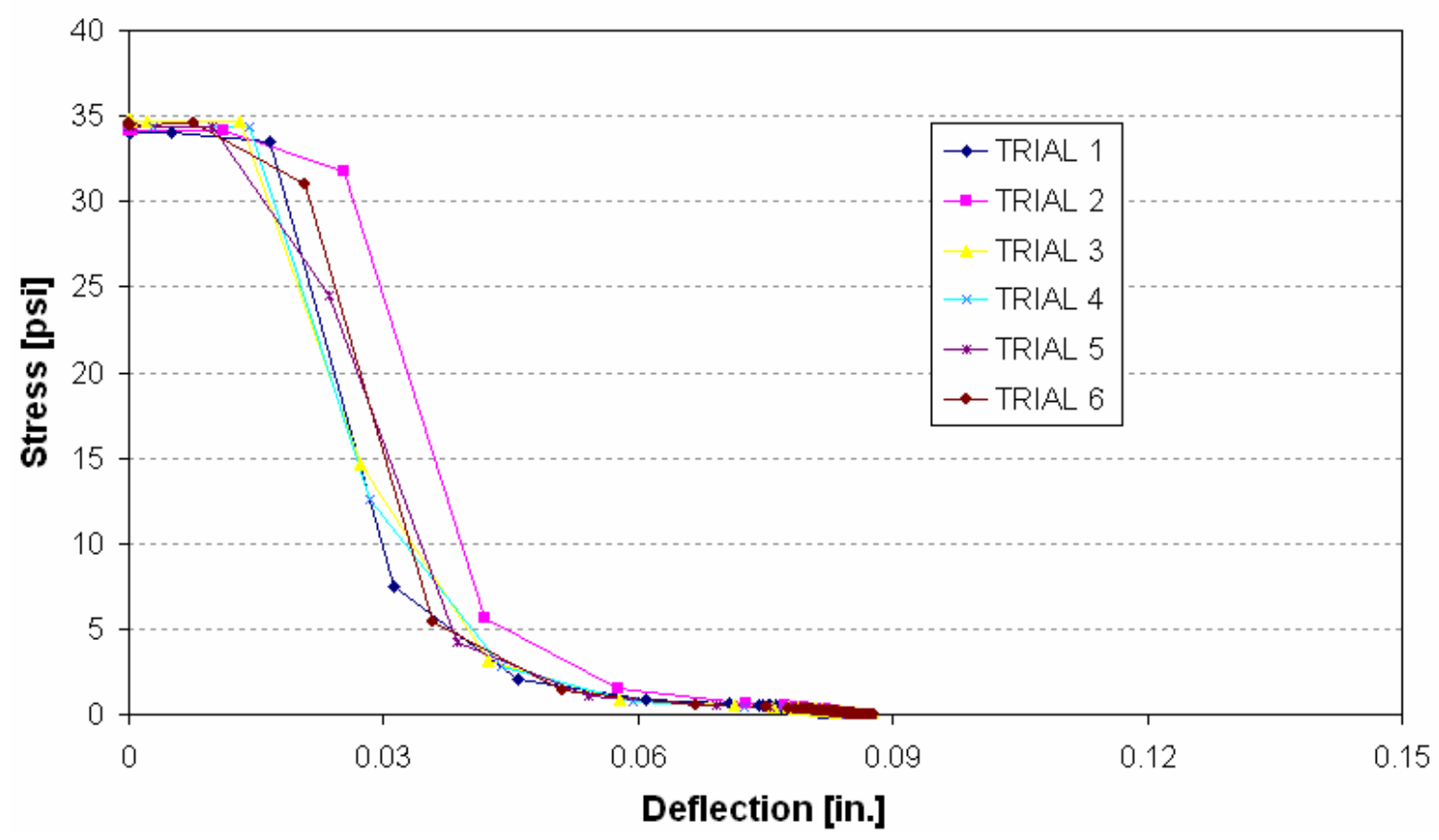

(a)

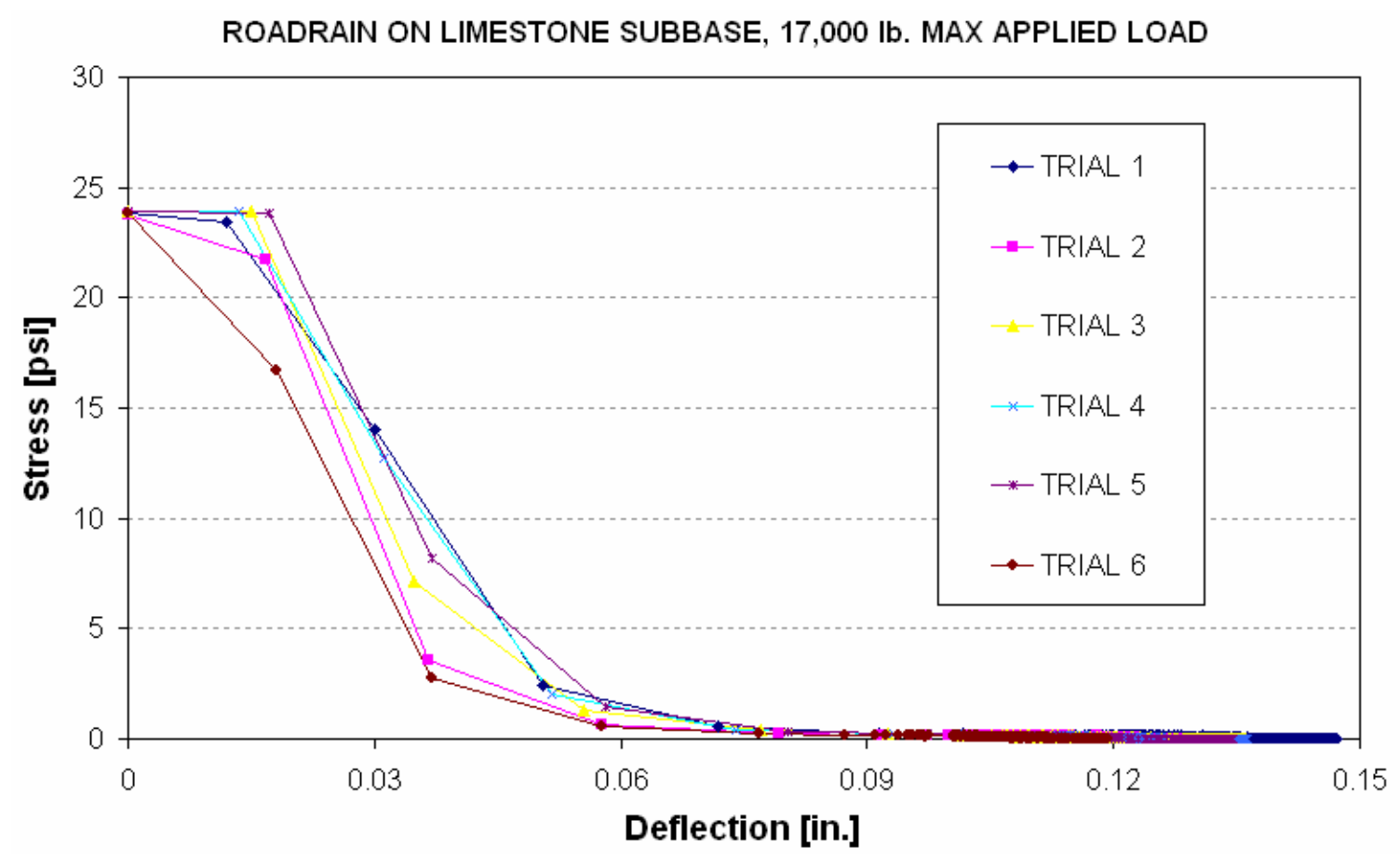

(b)

Figure B.5: Rebound Curves for Mid-Load Repetitive Static Plate Bearing Tests Using \#57 Limestone Aggregate Subbase: (a) Subbase Alone, (b) Subbase Plus Roadrain 
LIMESTONE SUBBASE, $50,000 \mathrm{lb}$. MAX APPLIED LOAD

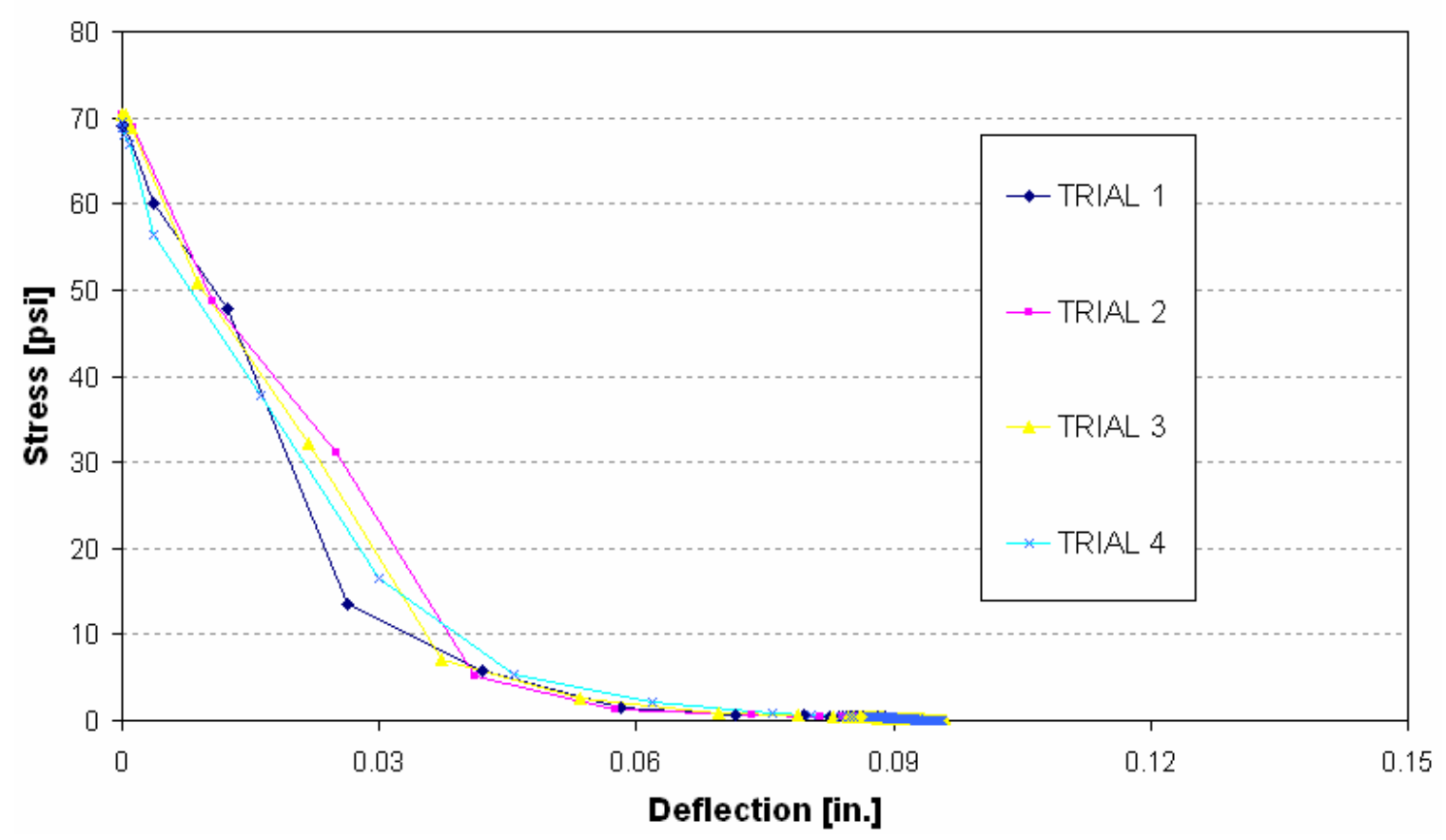

(a)

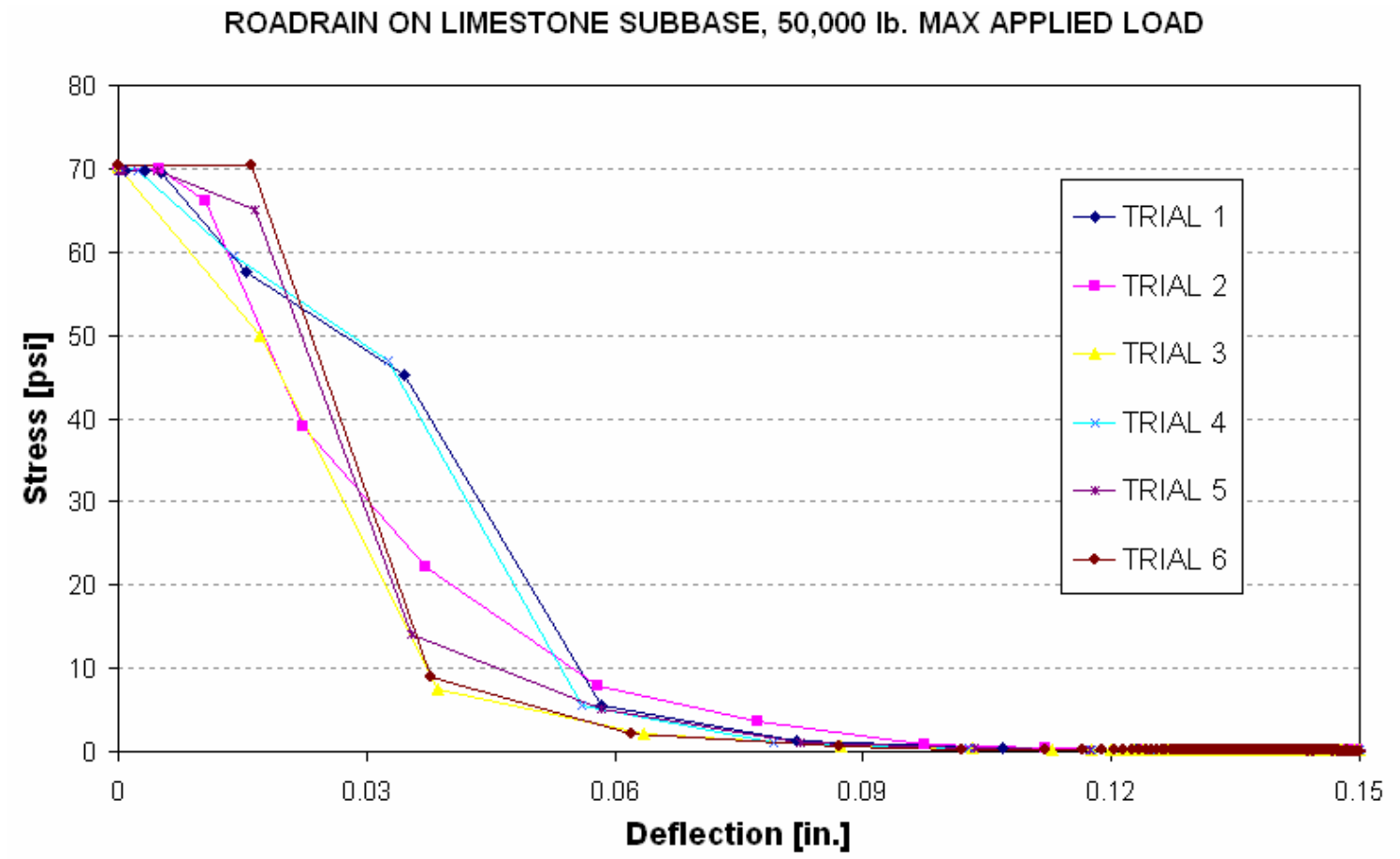

(b)

Figure B.6: Rebound Curves for Large-Load Repetitive Static Plate Bearing Tests Using \#57 Limestone Aggregate Subbase: (a) Subbase Alone, (b) Subbase Plus Roadrain 


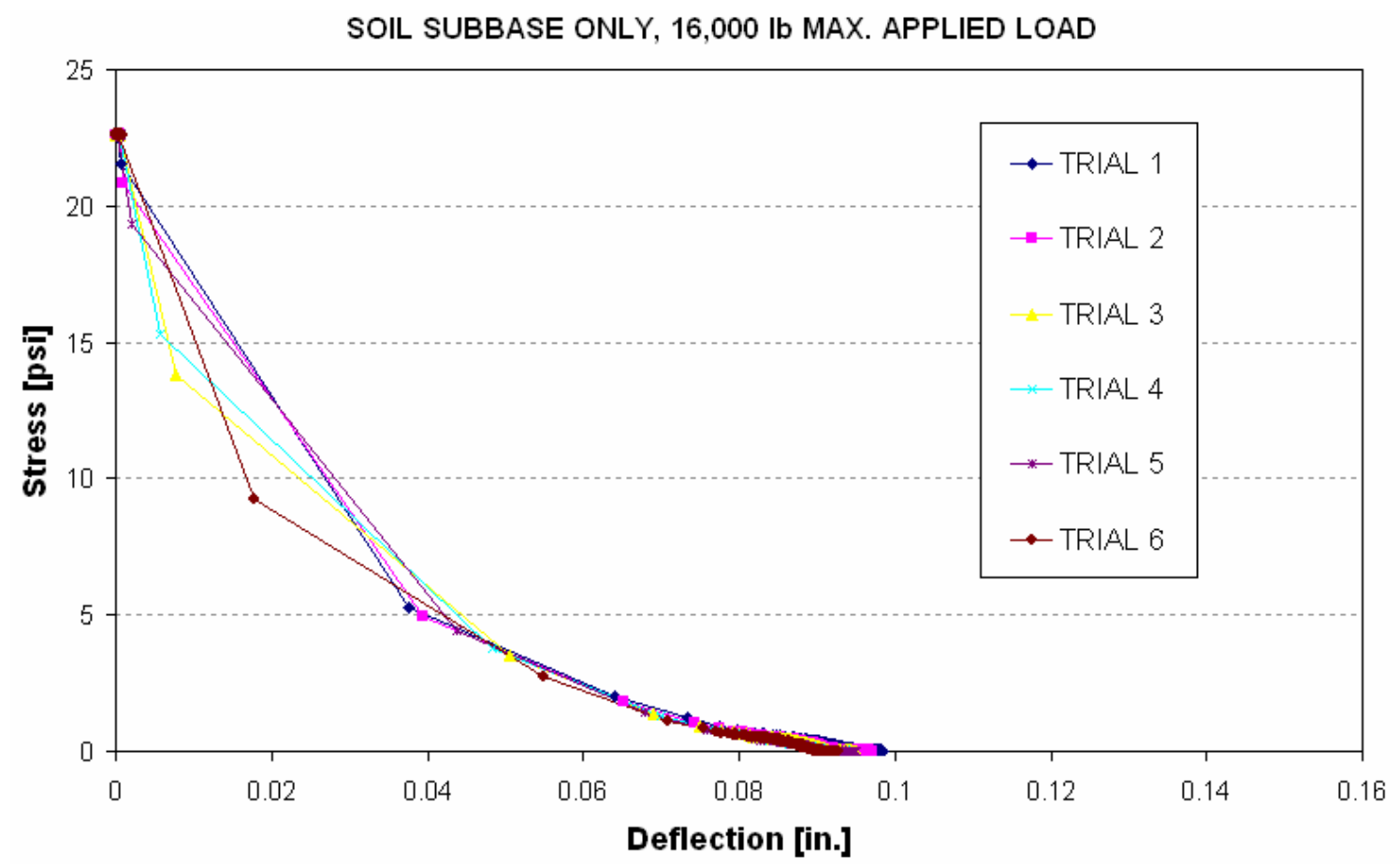

(a)

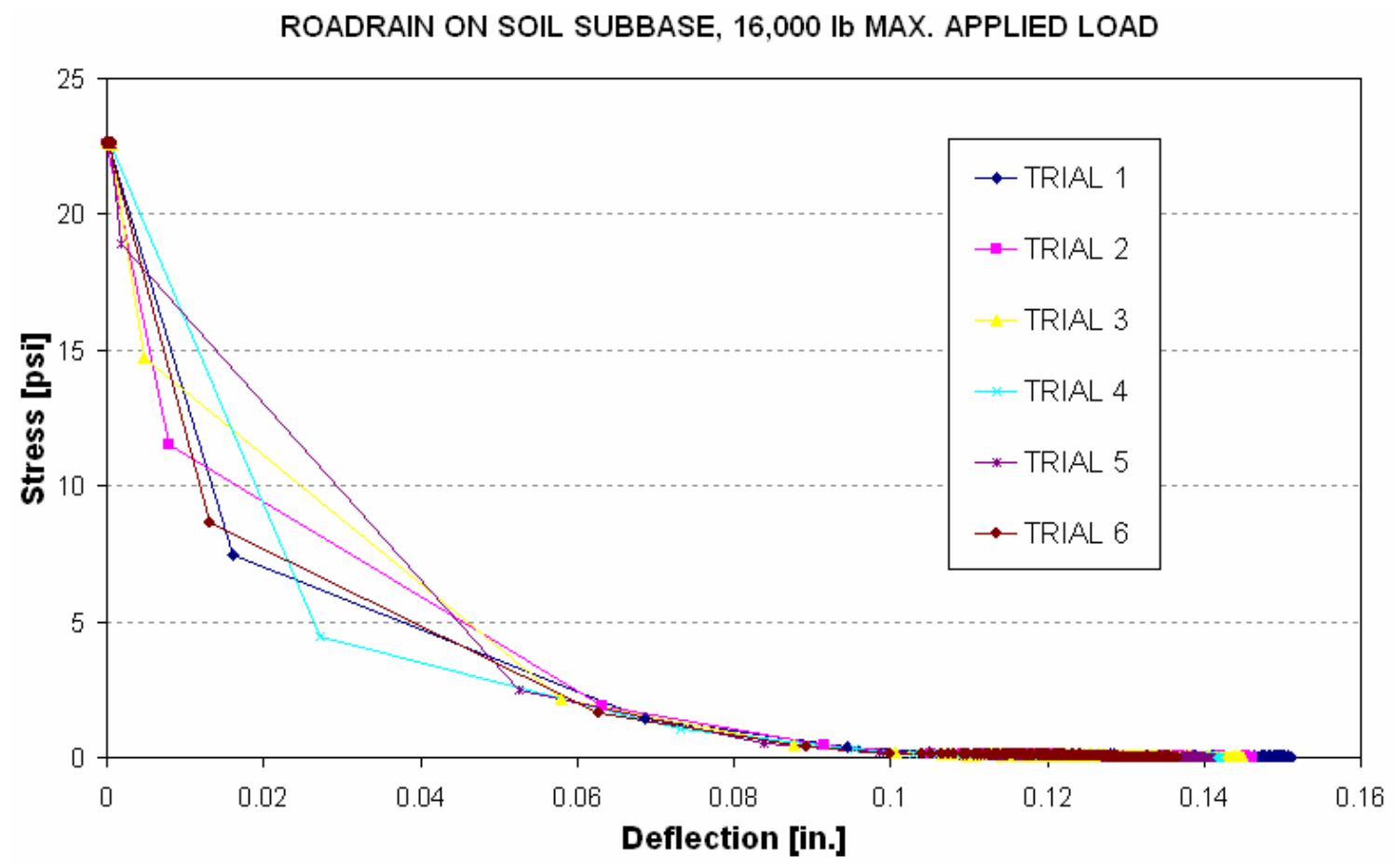

(b)

Figure B.7: Rebound Curves for Mid-Load Repetitive Static Plate Bearing Tests Using A-2-4 Soil Subbase: (a) Subbase Alone, (b) Subbase Plus Roadrain 


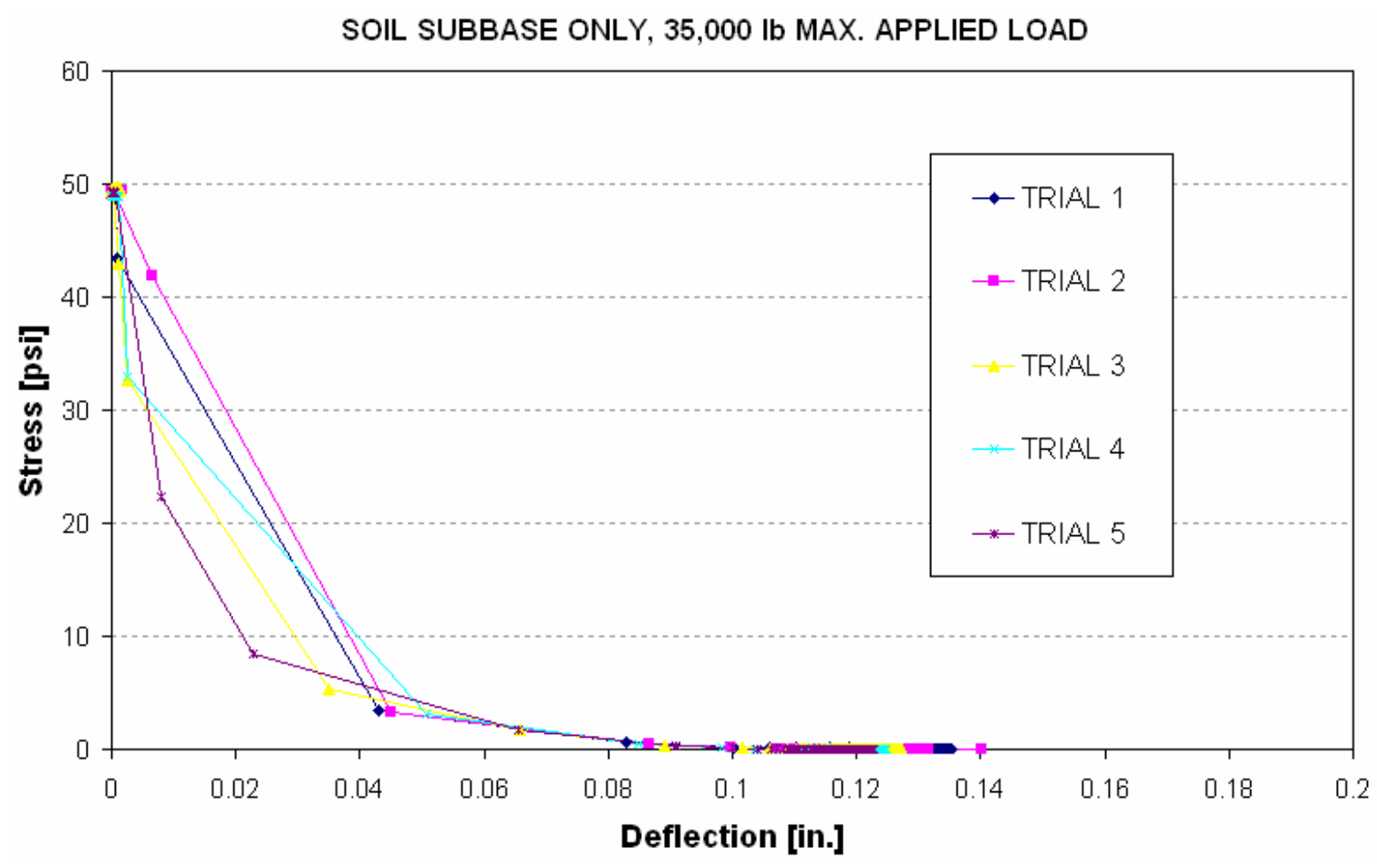

(a)



(b)

Figure B.8: Rebound Curves for Large-Load Repetitive Static Plate Bearing Tests Using A-2-4 Soil Subbase: (a) Subbase Alone, (b) Subbase Plus Roadrain

Digitally signed by John H. Hage $\mathrm{DN}$ : $\mathrm{Cn}=$ John $\mathrm{H}$. Hagen, o=West Virginia University Libraries, 